\title{
Equação de estimação generalizada \\ e influência local para modelos de regressão beta com medidas repetidas
}

\author{
Maria Kelly VenezUela
}

TESE APRESENTADA

$\mathrm{AO}$

INSTITUTO DE MATEMÁTICA E ESTATÍSTICA

DA

UNIVERSIDADE DE SÃO PAULO

PARA

OBTENÇÃO DO GRAU

$\mathrm{DE}$

DOUTOR EM CIÊNCIAS

Área de Concentração: Estatística

Orientadora: Profa. Dra. Mônica Carneiro Sandoval

Co-Orientadora: Profa. Dra. Denise Aparecida Botter

São Paulo, 04 de março de 2008. 



\section{Equação de estimação generalizada e influência local para modelos de regressão beta com medidas repetidas}

Este exemplar corresponde à redação final da tese devidamente corrigida e defendida por Maria Kelly Venezuela e aprovada pela comissão julgadora.

São Paulo, 04 de março de 2008.

Banca Examinadora:

- Profa. Dra. Mônica Sandoval (orientadora) - IME/USP

- Profa. Dra. Silvia Lopes de Paula Ferrari - IME/USP

- Profa. Dra. Clarice Garcia Borges Demétrio - ESALQ/USP

- Prof. Dr. Filidor Edilfonso Vilca Labra - UNICAMP

- Prof. Dr. Francisco Jose de Azevedo Cysneiros - UFPE 
"Uma pessoa permanece jovem na medida que ainda é capaz de aprender, adquirir novos hábitos e tolerar contradições". (Marie von Ebner-Eschenbach) 
Aos meus pais Batista e Terezinha, ao meu marido Gilberto e ao meu filho Enzo. 


\section{Agradecimentos}

Durante todo o tempo que me dediquei a este trabalho, contei com a ajuda e compreensão de muitas pessoas, entre as quais agradeço especialmente:

Às professoras Denise Aparecida Botter e Mônica Carneiro Sandoval pela orientação, confiança, apoio dedicados a mim e a este trabalho e, principalmente, pela amizade durante esses anos que trabalhamos juntas. Foi um imenso prazer tê-las como orientadoras, amigas, mães... Guardarei com carinho toda essa fase da minha vida que convivi, amadureci e aprendi muito com vocês. Obrigada por tudo!

À banca examinadora e ao professor Rinaldo Artes, pela leitura e correções sugeridas. Aos professores do IME-USP que participaram da minha formação acadêmica.

Ao meu marido Gilberto, pela compreensão, companheirismo, paciência e, novamente, pelo apoio sempre me mostrando que, no final, tudo iria valer a pena. E valeu! Ao meu filho Enzo que veio para trazer mais alegria e forças para terminar a tese.

Aos meus pais Batista e Terezinha, pelo amor, educação e paciência que nunca hesitaram em me oferecer. Ao meu irmão Sandro, em quem sempre me espelhei com relação aos estudos.

Às amizades de Iracema, Gisela, Patrícia e Raydonal, Michelli, Jacqueline e Juvêncio, Daniela e Marcos, Fred e Lílian, Cléber, Tatiana, Sumaia e Célia. À Bianca, Jacqueline e Marcos, por permanecermos amigos.

A todos os meus amigos e parentes que sempre me deram muitos motivos para rir e ser feliz.

Ao CNPq, pela bolsa de doutorado concedida, com a qual pude me dedicar com mais afinco a este trabalho. 


\section{Resumo}

Utilizando a teoria de função de estimação linear ótima (Crowder, 1987), propomos equações de estimação generalizadas para modelos de regressão beta (Ferrari e CribariNeto, 2004) com medidas repetidas. Além disso, apresentamos equações de estimação generalizadas para modelos de regressão simplex baseadas nas propostas de Song e Tan (2000) e Song et al. (2004) e equações de estimação generalizadas para modelos lineares generalizados com medidas repetidas baseadas nas propostas de Artes e J $\phi$ rgensen (2000) e Liang e Zeger (1986). Todas essas equações de estimação são desenvolvidas sob os enfoques da modelagem da média com homogeneidade da dispersão e da modelagem conjunta da média e da dispersão com intuito de incorporar ao modelo uma possível heterogeneidade da dispersão. Como técnicas de diagnóstico, desenvolvemos uma generalização de algumas medidas de diagnóstico quando abordamos quaisquer equações de estimação definidas tanto para modelagem do parâmetro de posição considerando a homogeneidade do parâmetro de dispersão como para modelagem conjunta dos parâmetros de posição e dispersão. Entre essas medidas, destacamos a proposta da influência local (Cook, 1986) desenvolvida para equações de estimação. Essa medida teve um bom desem-

penho, em simulações, para destacar corretamente pontos influentes. Por fim, realizamos aplicações a conjuntos de dados reais.

Palavras-chave: equação de estimação generalizada, distribuição beta, influência local. 


\section{Abstract}

Based on the concept of optimum linear estimating equation (Crowder, 1987), we develop generalized estimating equation (GEE) to analyze longitudinal data considering marginal beta regression models (Ferrari and Cribari-Neto, 2004). The GEEs are also presented to marginal simplex models for longitudinal continuous proportional data proposed by Song and Tan (2000) and Song et al. (2004) and to generalized linear models for longitudinal data based on the proposes of Artes and J $\phi$ rgensen (2000) and Liang and Zeger (1986). All of them are developed focusing the assumption of homogeneous dispersion and with varying dispersion. For the diagnostic techniques, we generalize some diagnostic measures for estimating equations to model the position parameter considering an homogeneous dispersion parameter and for joint modelling of position and dispersion parameters to take in account a possible heterogeneous dispersion. Among these measures, we point out the local influence (Cook, 1986) developed to estimating equations. This measure can correctly show influential observations in simulation study. Finally, the theory is applied to real data sets.

Keywords: generalized estimating equation, beta distribution, local influence. 


\section{Sumário}

1 Introdução $\quad \mathbf{1}$

1.1 Distribuição Beta . . . . . . . . . . . . . . . . . . . . 4

1.2 Distribuição Simplex . . . . . . . . . . . . . . . . . . . 7

1.3 Funções de estimação . . . . . . . . . . . . . . . . . . . . . . . 10

\section{Equações de Estimação Generalizadas}

2 Equações de estimação para modelos de regressão beta com medidas repetidas

2.1 Modelagem do parâmetro de posição . . . . . . . . . . . . . . . . 16

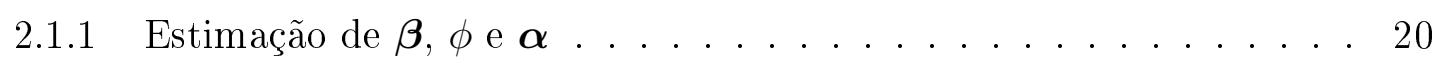

2.2 Modelagem conjunta dos parâmetros de posição e precisão . . . . . . . . . 23

2.2 .1 Estimação de $\boldsymbol{\beta}, \boldsymbol{\gamma}$ e $\boldsymbol{\alpha} \ldots \ldots \ldots 26$

3 Equações de estimação para modelos de regressão simplex com medidas $\begin{array}{ll}\text { repetidas } & \mathbf{2 8}\end{array}$

3.1 Modelagem do parâmetro de posição . . . . . . . . . . . . . . . . . 28

3.1 .1 Estimação de $\boldsymbol{\beta}, \phi$ e $\boldsymbol{\alpha} \ldots \ldots . \ldots . \ldots . \ldots . \ldots 31$

3.2 Modelagem conjunta dos parâmetros de posição e dispersão . . . . . . . . . 32

3.2 .1 Estimação de $\boldsymbol{\beta}, \boldsymbol{\gamma}$ e $\boldsymbol{\alpha} \ldots \ldots \ldots \ldots . \ldots \ldots$

4 Equações de estimação para modelos lineares generalizados com medidas repetidas

4.1 Modelagem do parâmetro de posição . . . . . . . . . . . . . . 36

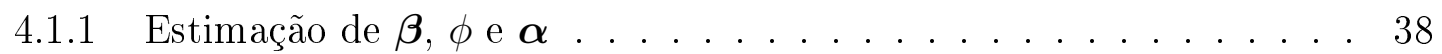

4.2 Modelagem conjunta dos parâmetros de posição e dispersão . . . . . . . . . 39

4.2 .1 Estimação de $\boldsymbol{\beta}, \boldsymbol{\gamma}$ e $\boldsymbol{\alpha}$. . . . . . . . . . . . . . . . . . . . 42 
5 Algumas medidas de diagnóstico para dados com medidas repetidas 44

5.1 Pontos alavanca, influentes e aberrantes . . . . . . . . . . . . . 45

5.2 Seleção de modelos e de matriz de correlação de trabalho . . . . . . . . . . 49

6 Influência local para equações de estimação 51

6.1 Influência local generalizada . . . . . . . . . . . . . . . . . 52

6.2 Influência local para equações de estimação . . . . . . . . . . . . . . . 53

6.3 Esquemas de perturbação sob homogeneidade da dispersão . . . . . . . . . 56

6.3.1 Ponderação de casos . . . . . . . . . . . . . . . . . . 57

6.3.2 Perturbação da variável resposta . . . . . . . . . . . . . 57

6.3.3 Perturbação individual das covariáveis . . . . . . . . . . . . . . 59

6.3.4 Perturbação do parâmetro de precisão . . . . . . . . . . . . . 63

6.3.5 Perturbação na matriz de correlação de trabalho $\mathbf{R}(\boldsymbol{\alpha})$. . . . . . . 65

6.4 Esquemas de perturbação sob heterogeneidade da dispersão . . . . . . . . . 66

6.4.1 Ponderação de casos . . . . . . . . . . . . . . . . . . . 67

6.4 .2 Perturbação da variável resposta . . . . . . . . . . . . . . 67

6.4.3 Perturbação individual das covariáveis . . . . . . . . . . . . . . . 69

\section{Simulações e Aplicações $\quad 80$}

7 Aplicações a dados simulados $\quad \mathbf{8 1}$

7.1 Simulação I - Dados com distribuição Normal . . . . . . . . . . . . . . . 81

7.2 Simulação II - Dados com distribuição de Poisson . . . . . . . . . . . . . . 89

8 Aplicações a dados reais $\quad 98$

8.1 Aplicação I - Estudo de oftalmologia . . . . . . . . . . . . . . . . . . 98

8.2 Aplicação II - Estudo de desenvolvimento motor de bebês . . . . . . . . . . 113

9 Conclusões e Estudos futuros $\quad 129$

Apêndice A Detalhes para obtenção das equações de estimação 132

A.1 Modelos de regressão beta . . . . . . . . . . . . . . . . . . . . . 132

A.1.1 EEGs sob homogeneidade da dispersão . . . . . . . . . . . . . . . 132

A.1.2 EEGs sob heterogeneidade da dispersão . . . . . . . . . . . . . . . 133

A.2 Modelos de regressão simplex . . . . . . . . . . . . . . . . 135

A.2.1 EEGs sob homogeneidade da dispersão . . . . . . . . . . . . . 135

A.2.2 EEGs sob heterogeneidade da dispersão . . . . . . . . . . . . . 135 
A.3 Modelos lineares generalizados . . . . . . . . . . . . . . . . 137

A.3.1 EEGs sob heterogeneidade da dispersão . . . . . . . . . . . . . . 137

Apêndice B Detalhes para obtenção das medidas de influência local 139

B.1 Influência local sob homogeneidade da dispersão . . . . . . . . . . . . . 139

B.1.1 Perturbação da variável resposta . . . . . . . . . . . . . . . 139

B.1.2 Perturbação individual de covariáveis . . . . . . . . . . . . . . . . 141

B.1.3 Perturbação no parâmetro de precisão . . . . . . . . . . . . . . . 142

B.2 Influência local sob heterogeneidade da dispersão . . . . . . . . . . . . . . 143

B.2.1 Perturbação individual de covariáveis . . . . . . . . . . . . . . . . 143

$\begin{array}{lll}\text { Apêndice C } & \text { Tabelas } & 147\end{array}$ 


\section{Lista de Tabelas}

8.1 Estimativas e erros padrões dos parâmetros do modelo de regressão beta sob homogeneidade da dispersão e com estrutura AR-1. Estudo de oftalmologia.100

8.2 Estimativas e erros padrões dos parâmetros do modelo de regressão beta sob heterogeneidade da dispersão e com estrutura AR-1. Estudo de oftalmologia.104

8.3 Estimativas e erros padrões dos parâmetros do modelo de regressão simplex sob homogeneidade da dispersão e com estrutura AR-1. Estudo de oftalmologia. . . . . . . . . . . . . . . . . . . . 110

8.4 Estimativas e erros padrões dos parâmetros do modelo de regressão simplex sob heterogeneidade da dispersão e com estrutura AR-1. Estudo de oftalmologia. . . . . . . . . . . . . . . . . . . . . . . 113

8.5 Estimativas e erros padrões dos parâmetros do modelo de regressão beta sob homogeneidade da dispersão e com estrutura AR-1. Estudo de desenvolvimento motor de bebês. . . . . . . . . . . . . . . . . . . . . . . . . 119

8.6 Estimativas e erros padrões dos parâmetros do modelo de regressão beta sob heterogeneidade da dispersão e com estrutura AR-1. Estudo de desenvolvimento motor de bebês. . . . . . . . . . . . . . . . . . . . . . . . . . 121

8.7 Estimativas e erros padrões dos parâmetros do modelo de regressão simplex sob homogeneidade da dispersão e com estrutura AR-1. Estudo de desenvolvimento motor de bebês. . . . . . . . . . . . . . . . . . . . 124

8.8 Estimativas e erros padrões dos parâmetros do modelo de regressão simplex sob heterogeneidade da dispersão e com estrutura AR-1. Estudo de desenvolvimento motor de bebês. . . . . . . . . . . . . . 126

C.1 Funções $\mathbf{G}$ e F de acordo com a função de ligação adotada . . . . . . . . 147

C.2 Algumas distribuições pertencentes à família exponencial e respectivos resultados . . . . . . . . . . . . . . . . . . . . 148

C.3 Funções $c(\mathrm{y} ; \phi), \dot{c}(\mathrm{y} ; \phi)$ e $\ddot{c}(\mathrm{y} ; \phi)$ de acordo com a distribuição adotada . . . 149

C.4 Alguns resultados para influência local quando abordamos MLGs . . . . . 149 


\section{Lista de Figuras}

7.1 Valores simulados de uma Normal considerando estrutura padrão uniforme $\operatorname{com} \alpha=0,3$ (a) e com $\alpha=0,9$ (b) e estrutura AR-1 com $\alpha=0,3$ (c) e com $\alpha=0,9$ (d). Simulação Normal sob homogeneidade da dispersão. . . . 84

7.2 Gráficos de influência local considerando a verdadeira matriz de correlação (com estrutura padrão uniforme e $\alpha=0,3$ ) e com matriz de correlação de trabalho padrão uniforme, AR-1 e independente. Simulação Normal sob homogeneidade da dispersão. . . . . . . . . . . . . . . . . . . . . 85

7.3 Gráficos de influência local considerando a verdadeira matriz de correlação (com estrutura padrão uniforme e $\alpha=0,9$ ) e com matriz de correlação de trabalho padrão uniforme, AR-1 e independente. Simulação Normal sob homogeneidade da dispersão. . . . . . . . . . . . . . . . . . 86

7.4 Gráficos de influência local considerando a verdadeira matriz de correlação (com estrutura AR-1 e $\alpha=0,3$ ) e com matriz de correlação de trabalho padrão uniforme, AR-1 e independente. Simulação Normal sob homogeneidade da dispersão.

7.5 Gráficos de influência local considerando a verdadeira matriz de correlação (com estrutura AR-1 e $\alpha=0,9$ ) e com matriz de correlação de trabalho padrão uniforme, AR-1 e independente. Simulação Normal sob homogeneidade da dispersão. . . . . . . . . . . . . . . . . . . . . . . . . . 88

7.6 Valores simulados de uma Normal sob heterogeneidade da dispersão e estrutura padrão uniforme com $\alpha=0,9$ (a), medidas de influência local com esquema de perturbação ponderação de casos considerando a verdadeira correlação (b), padrão uniforme (c), AR-1 (d) e independente (e). Simulação Normal sob heterogeneidade da dispersão. . . . . . . . . . . . . . . . . 90

7.7 Valores simulados de uma Normal sob heterogeneidade da dispersão e estrutura AR-1 com $\alpha=0,9$ (a), medidas de influência local com esquema de perturbação ponderação de casos considerando a verdadeira correlação (b), padrão uniforme (c), AR-1 (d) e independente (e). Simulação Normal sob heterogeneidade da dispersão. 
7.8 Valores simulados de uma Poisson considerando estrutura padrão uniforme $\operatorname{com} \alpha=0,3$ (a) e com $\alpha=0,6$ (b) e estrutura AR-1 com $\alpha=0,3$ (c) e com $\alpha=0,6$ (d). Simulação Poisson. . . . . . . . . . . . . . . . . 93

7.9 Gráficos de influência local considerando a verdadeira matriz de correlação (com estrutura padrão uniforme e $\alpha=0,3$ ) e com matriz de correlação de trabalho padrão uniforme, AR-1 e independente. Simulação Poisson. . . . . 94

7.10 Gráficos de influência local considerando a verdadeira matriz de correlação (com estrutura padrão uniforme e $\alpha=0,6$ ) e com matriz de correlação de trabalho padrão uniforme, AR-1 e independente. Simulação Poisson. . . . . 95

7.11 Gráficos de influência local considerando a verdadeira matriz de correlação (com estrutura AR-1 e $\alpha=0,3$ ) e com matriz de correlação de trabalho padrão uniforme, AR-1 e independente. Simulação Poisson. . . . . . . . . . 96

7.12 Gráficos de influência local considerando a verdadeira matriz de correlação (com estrutura AR-1 e $\alpha=0,6$ ) e com matriz de correlação de trabalho padrão uniforme, AR-1 e independente. Simulação Poisson. . . . . . . . . . 97

8.1 Gráfico de dispersão da porcentagem de gás versus dias após a cirurgia, para as concentrações de gás iguais a 15, 20 e 25. Estudo de oftalmogia. . . 99

8.2 Curvas ajustadas e gráficos de diagnóstico com todas as observações (a)(d) e sem as observações $(2,1),(16,10)$ e $(25,20)$ (e)-(h), para modelo de regressão beta sob homogeneidade da dispersão. Estudo de oftalmologia. . 102

8.3 Gráficos de influência local para $\boldsymbol{\beta}$ considerando todas as observações (a)(d) e sem as observações $(2,1),(16,10)$ e $(25,20)$ (e)-(h), para modelo de regressão beta sob homogeneidade da dispersão. Estudo de oftalmologia. . 103

8.4 Curvas ajustadas (a); gráficos de diagnóstico do ajuste de $\boldsymbol{\beta}$ (b)-(d) e do ajuste de $\gamma(\mathrm{e})-(\mathrm{g})$, para modelos de regressão beta sob heterogeneidade da dispersão e com todas as observações. Estudo de oftalmologia. . . . . . . . 105

8.5 Gráficos de influência local para $\boldsymbol{\theta}$ (a)-(c); para $\boldsymbol{\beta}(\mathrm{d})$-(f) e para $\boldsymbol{\gamma}(\mathrm{g})$-(i), para modelos de regressão beta sob heterogeneidade da dispersão e com todas as observações. Estudo de oftalmologia. . . . . . . . . . . . . . . 106

8.6 Curvas ajustadas (a); gráficos de diagnóstico do ajuste de $\boldsymbol{\beta}$ (b)-(d) e do ajuste de $\gamma(\mathrm{e})-(\mathrm{g})$, para modelos de regressão beta sob heterogeneidade da dispersão e sem as observações $(2,1)$, (16,10) e $(25,20)$. Estudo de oftalmologia. . . . . . . . . . . . . . . . . . . . . 108

8.7 Gráficos de influência local para $\boldsymbol{\theta}(\mathrm{a})-(\mathrm{c})$; para $\boldsymbol{\beta}(\mathrm{d})$-(f) e para $\boldsymbol{\gamma}(\mathrm{g})$-(i), para modelos de regressão beta sob heterogeneidade da dispersão e sem as observações $(2,1),(16,10)$ e $(25,20)$. Estudo de oftalmologia. . . . . . . . . 109 
8.8 Curvas ajustadas e gráficos de diagnóstico com todas as observações (a)-(d) e sem as observações $(16,10)$ e $(25,20)$ (e)-(h), para modelo de regressão simplex sob homogeneidade da dispersão. Estudo de oftalmologia. . . . . . 111

8.9 Gráficos de influência local para $\boldsymbol{\beta}$ considerando todas as observações (a)(d) e sem as observações $(16,10)$ e $(25,20)$ (e)-(h), para modelo de regressão simplex sob homogeneidade da dispersão. Estudo de oftalmologia. . . . . . 112

8.10 Curvas ajustadas (a); gráficos de diagnóstico do ajuste de $\boldsymbol{\beta}$ (b)-(d) e do ajuste de $\gamma(\mathrm{e})-(\mathrm{g})$, para modelos de regressão simplex sob heterogeneidade da dispersão e com todas as observações. Estudo de oftalmologia. . . . . . 114

8.11 Gráficos de influência local para $\boldsymbol{\theta}$ (a)-(c); para $\boldsymbol{\beta}(\mathrm{d})$-(f) e para $\boldsymbol{\gamma}(\mathrm{g})$-(i), para modelos de regressão simplex sob heterogeneidade da dispersão e com todas as observações. Estudo de oftalmologia. . . . . . . . . . . . . . 115

8.12 Curvas ajustadas (a); gráficos de diagnóstico do ajuste de $\boldsymbol{\beta}$ (b)-(d) e do ajuste de $\gamma(\mathrm{e})-(\mathrm{g})$, para modelos de regressão simplex sob heterogeneidade da dispersão e sem as observações $(9,12),(16,10)$ e $(25,20)$. Estudo de oftalmologia. . . . . . . . . . . . . . . . . . . 116

8.13 Gráficos de influência local para $\boldsymbol{\theta}$ (a)-(c); para $\boldsymbol{\beta}(\mathrm{d})$-(f) e para $\boldsymbol{\gamma}(\mathrm{g})$-(i), para modelos de regressão simplex sob heterogeneidade da dispersão e sem as observações $(9,12),(16,10)$ e $(25,20)$. Estudo de oftalmologia. . . . . . . 117

8.14 Gráfico de dispersão da porcentagem de escore versus idade corrigida, para os grupos A termo e Pré-termo. Estudo de desenvolvimento motor de bebês.118

8.15 Curvas ajustadas e gráficos de diagnóstico com todas as observações, para modelo de regressão beta sob homogeneidade da dispersão. Estudo de desenvolvimento motor de bebês. . . . . . . . . . . . . . . . . . . . 120

8.16 Curvas ajustadas (a); gráficos de diagnóstico do ajuste de $\boldsymbol{\beta}$ (b)-(d) e do ajuste de $\gamma(\mathrm{e})-(\mathrm{g})$, para modelos de regressão beta sob heterogeneidade da dispersão e com todas as observações. Estudo de desenvolvimento motor de bebês. . . . . . . . . . . . . . . . . . . . . . . . . . . 122

8.17 Gráficos de influência local para $\boldsymbol{\theta}(\mathrm{a})-(\mathrm{c})$; para $\boldsymbol{\beta}(\mathrm{d})$-(f) e para $\boldsymbol{\gamma}(\mathrm{g})$-(i), para modelos de regressão beta sob heterogeneidade da dispersão e com todas as observações. Estudo de desenvolvimento motor de bebês. . . . . . 123

8.18 Curvas ajustadas e gráficos de diagnóstico com todas as observações, para modelo de regressão simplex sob homogeneidade da dispersão. Estudo de desenvolvimento motor de bebês. . . . . . . . . . . . . . . . . . . 125 
8.19 Curvas ajustadas (a); gráficos de diagnóstico do ajuste de $\boldsymbol{\beta}$ (b)-(d) e do ajuste de $\gamma(\mathrm{e})-(\mathrm{g})$, para modelos de regressão simplex sob heterogeneidade da dispersão e com todas as observações. Estudo de desenvolvimento motor de bebês. . . . . . . . . . . . . . . . . . . . . . . 127

8.20 Gráficos de influência local para $\boldsymbol{\theta}$ (a)-(c); para $\boldsymbol{\beta}(\mathrm{d})$-(f) e para $\boldsymbol{\gamma}(\mathrm{g})$-(i), para modelos de regressão simplex sob heterogeneidade da dispersão e com todas as observações. Estudo dos bebês. . . . . . . . . . . . . . 128 


\section{Capítulo 1}

\section{Introdução}

Experimentos com medidas repetidas são freqüentes em quase todos os campos científicos, sendo cada unidade experimental submetida a diferentes condições experimentais de forma aleatória, quando não devem seguir necessariamente uma ordem de aplicação, ou de forma longitudinal, na qual não se pode aleatorizar a ordem dos tratamentos aplicados em cada unidade experimental. Esse tipo de planejamento, embora tenha como principal desvantagem o acompanhamento das unidades experimentais durante a realização do estudo, têm como principais vantagens os seguintes fatos: requerem menos unidades experimentais do que planejamentos completamente casualizados; proporcionam condições mais adequadas para o estudo de covariáveis que possam ter influência na variável resposta; melhoram, em geral, a precisão de contrastes associados às diferenças entre os valores médios da variável resposta de diferentes tratamentos; e permitem o estudo da mudança do comportamento da resposta média da unidade experimental nos diferentes tratamentos (incorpora informação sobre a variação individual na análise).

Para análise de dados com medidas repetidas, existe uma grande variedade de técnicas quando a variável resposta segue uma distribuição normal. Entre elas, podemos destacar a análise de variância com medidas repetidas (Neter et al., 1996), análise uni/multivariada de perfis, análise de curvas de crescimento (Singer e Andrade, 1986) e modelos normais com efeitos aleatórios (Laird e Ware, 1982; Andreoni, 1989). O fato de existir uma versão multivariada da distribuição normal com boas propriedades do ponto de vista inferencial facilita o estudo desses modelos.

Abandonando a suposição de normalidade da variável resposta, uma série de dificuldades pode surgir devido à escassez de distribuições multivariadas alternativas com tais propriedades. Justamente por esse motivo, não são muitas as técnicas existentes na literatura para se trabalhar com distribuições não-normais levando em consideração a 
dependência entre observações da mesma unidade experimental.

Zeger e Liang (1986) desenvolveram equações de estimação generalizadas (EEGs) usando os modelos de quasi-verossimilhança (Wedderburn, 1974) para analisar dados longitudinais. Liang e Zeger (1986) derivaram essas EEGs num contexto um pouco mais restrito. Eles assumem que a distribuição marginal da variável dependente segue um modelo linear generalizado (MLG) desenvolvido por Nelder e Wedderburn (1972). Em ambos os artigos, as EEGs são construídas sem a especificação da distribuição conjunta e uma matriz de correlação de trabalho para o vetor de medidas repetidas de cada unidade experimental deve ser especificada, a qual não precisa ser correta para se obter consistência dos parâmetros de regressão. Prentice e Zhao (1991) estendem a proposta de Liang e Zeger (conhecida como EEG1), estimando os parâmetros de correlação também via equações de estimação. Na literatura, a proposta de Prentice e Zhao é conhecida como EEG2.

Depois dessas propostas, as equações de estimação também passaram a ser utilizadas para modelar o parâmetro de dispersão (denominada EEG3 segundo proposta, por exemplo, de Song et al. (2004)), já que na prática, a suposição de homogeneidade da dispersão pode ser questionada. Além disso, as equações de estimação têm sido estendidas para outras classes de modelos que englobam outras distribuições, como é o caso dos modelos de dispersão desenvolvidos por J $\phi$ rgensen (1997). Essa classe de modelos se tornou importante, principalmente, por conter a distribuição von Mises, adequada para modelar dados circulares; e a distribuição simplex, para modelar percentagens ou proporções. Artes (1997) desenvolveu equações de estimação, na proposta de EEG1, EEG2 e EEG3, para a classe de modelos de dispersão com medidas repetidas e apresenta uma aplicação para dados circulares utilizando a distribuição von Mises. Song e Tan (2000) apresentam equações de estimação no contexto de EEG2 usando apenas a distribuição simplex e julgam ter resultados melhores por utilizarem propriedades específicas dessa distribuição. Considerando apenas a distribuição simplex, Song et al. (2004) apresentam as EEG3s e mostram, por simulação, como a modelagem do parâmetro de dispersão pode alterar as estimativas e diminuir os desvios padrões dos parâmetros de regressão que modelam a média, quando comparada ao ajuste que supõe-se homogeneidade da dispersão.

Para situações em que a variável resposta se distribui continuamente no intervalo $(0,1)$ e é explicada por outras variáveis por meio de uma estrutura de regressão, também se encontra um modelo de regressão beta proposto por Ferrari e Cribari-Neto (2004). Os autores propõem uma parametrização para a distribuição beta que permite a modelagem da média envolvendo também o parâmetro de precisão. Assim como a distribuição simplex, a distribuição beta é utilizada para modelar proporções, com a vantagem da sua densidade assumir diferentes formas dependendo dos dois parâmetros que indexam a distribuição. 
Ospina (2007) propõe os modelos de regressão beta com dispersão variável quando há independência entre todas as observações e desenvolve medidas de diagnóstico capazes de captar a presença dessa dispersão variável nos dados.

Em nosso trabalho, assumindo que a variável dependente tem distribuição marginal beta e há mais do que uma observação realizada numa mesma unidade experimental, estendemos as propostas de EEG1 e EEG3 para os modelos de regressão beta (Ferrari e Cribari-Neto, 2004) com medidas repetidas. Além disso, desenvolvemos algumas medidas de diagnóstico para avaliar a adequação de um modelo ajustado aos dados.

Dessa forma, este trabalho está dividido em três partes, as quais visam, respectivamente, desenvolver: equações de estimação para modelos de regressão com medidas repetidas (Capítulos 2 ao 4); técnicas de diagnóstico (Capítulos 5 e 6); e simulações e aplicações (Capítulos 7 e 8). Antes de apresentá-las, com o intuito de facilitar a leitura do texto, temos as Seções 1.1 e 1.2 que descrevem, de forma suscinta, a distribuição beta de acordo com a parametrização utilizada por Ferrari e Cribari-Neto (2004) e a distribuição simplex desenvolvida por Barndorff-Nielsen e J $\phi$ rgensen (1991). Na Seção 1.3, introduzimos alguns conceitos sobre funções de estimação.

No Capítulo 2, propomos equações de estimação para modelos de regressão beta com medidas repetidas. Essas equações são construídas para modelagem da média supondo homogeneidade da dispersão e modelagem conjunta da média e da dispersão. O Capítulo 3 apresenta equações de estimação generalizadas para modelos de regressão simplex mesclando resultados desenvolvidos por Artes e J $\phi$ rgensen (2000), Song e Tan (2000) e Song et al. (2004). Essas equações também são construídas para modelos homoscedásticos (modelagem da média supondo homogeneidade da dispersão) e heteroscedásticos (modelagem conjunta da média e da dispersão). Para finalizar essa primeira parte do trabalho, o Capítulo 4 contém equações de estimação para modelos lineares generalizados considerando as propostas de Liang e Zeger (1986) e de Artes e J $\phi$ rgensen (2000). Essas equações são escritas considerando alguns resultados específicos da família exponencial. Esse capítulo se fez necessário, principalmente, para o desenvolvimento das simulações apresentadas na terceira parte.

Na segunda parte deste trabalho, propomos uma generalização de algumas medidas de diagnóstico quando abordamos quaisquer equações de estimação para dados com medidas repetidas definidas tanto para modelagem do parâmetro de posição considerando a homogeneidade do parâmetro de dispersão como para modelagem conjunta dos parâmetros de posição e dispersão. As medidas desenvolvidas são a matriz de projeção utilizada para detectar pontos alavanca (observações influentes na matriz de covariáveis), a distância de Cook utilizada para detectar pontos influentes no vetor de respostas e os resíduos do mo- 
delo, para detectar pontos aberrantes ("outliers"), as quais estão definidas para os modelos lineares generalizados em Paula (2004) e para modelos lineares generalizados com medidas repetidas em Venezuela et al. (2007). Preisser e Perin (2007) propõem medidas de diagnóstico para os parâmetros de correlação quando esses são modelados conjuntamente com a média via equações de estimação. A modelagem da correlação não é tratada neste trabalho.

No Capítulo 6, propomos medidas de influência local para equações de estimação generalizadas baseadas nas propostas de Cook (1986) e Cadigan e Farrell (2002). As medidas foram desenvolvidas para os esquemas de perturbação ponderação de casos, da variável resposta, de uma covariável contínua da matriz de planejamento, do parâmetro de precisão e, finalmente, na matriz de correlação de trabalho. Todos esses esquemas foram tratados para modelagem da média sob homogeneidade do parâmetro de dispersão e os três primeiros, para modelagem conjunta da média e do parâmetro de dispersão.

Por fim, na terceira parte, temos algumas aplicações a dados simulados, cujo principal objetivo é avaliar o impacto que a escolha de uma estrutura de correlação causa nas medidas de influência local quando essa difere ou não da verdadeira matriz de correlação que gerou os dados. No Capítulo 8, apresentamos duas aplicações a dados reais ajustados com o uso das equações de estimação desenvolvidas na primeira parte e validados com as técnicas de diagnóstico propostas na Parte II.

De maneira geral, esta tese traz como inédito os temas desenvolvidos nos Capítulos 2 e 6 . O Capítulo 3 compacta vários resultados descritos na literatura e seu desenvolvimento julgamos necessário neste trabalho por se tratar da distribuição simplex que, assim como a distribuição beta tratada no Capítulo 2, tem o suporte definido no intervalo unitário $(0,1)$. A principal necessidade do Capítulo 4 é para o desenvolvimento do Capítulo 7 que simula dados com distribuições marginais correlacionadas pertencentes à família exponencial. Para realizar os ajustes descritos nos Capítulos 7 e 8, desenvolvemos rotinas no software R versão 2.5.1 que se encontra disponível gratuitamente no endereço http://www.r-project.org/.

\subsection{Distribuição Beta}

Nesta seção, apresentamos alguns resultados inferenciais sobre a distribuição beta utilizando a parametrização definida por Ferrari e Cribari-Neto (2004). Para maiores detalhes desta distribuição, veja também Oliveira (2004) que apresenta um estudo sobre o comportamento assintótico dos estimadores e de testes de hipóteses no modelo de regressão beta. 
A família de distribuições beta é composta de todas as distribuições com função densidade de probabilidade da variável aleatória y escritas na forma

$$
p(\mathrm{y} ; p, q)=\frac{\Gamma(p+q)}{\Gamma(p) \Gamma(q)} \mathrm{y}^{p-1}(1-\mathrm{y})^{q-1},
$$

com $0<\mathrm{y}<1, p>0$ e $q>0$. A função gama $\Gamma(\cdot)$ avaliada no ponto $\lambda$ é dada por

$$
\Gamma(\lambda)=\int_{0}^{\infty} \mathrm{y}^{\lambda-1} e^{-\mathrm{y}} d \mathrm{y}
$$

A média e a variância de y são dadas por

$$
\mathrm{E}(\mathrm{y})=\frac{p}{p+q} \quad \text { e } \quad \operatorname{Var}(\mathrm{y})=\frac{p q}{(p+q)^{2}(p+q+1)} .
$$

Essa distribuição não apresenta a estrutura dos modelos de locação-escala, pois ambos os parâmetros, $p$ e $q$, são de forma. Ferrari e Cribari-Neto (2004) sugerem uma nova parametrização de (1.1) com o intuito de obter parâmetros de posição e precisão, representados por $\mu$ e $\phi$, respectivamente. Essa reparametrização é dada por

$$
\mu=\frac{p}{(p+q)} \quad \text { e } \quad \phi=p+q .
$$

Assim, a densidade (1.1) reescrita como uma família de distribuições beta com parâmetro de posição $\mu$ e parâmetro de precisão $\phi, \mathcal{B}(\mu, \phi)$, é dada por

$$
p(\mathrm{y} ; \mu, \phi)=\frac{\Gamma(\phi)}{\Gamma(\mu \phi) \Gamma((1-\mu) \phi)} \mathrm{y}^{\mu \phi-1}(1-\mathrm{y})^{(1-\mu) \phi-1},
$$

com $0<\mathrm{y}<1,0<\mu<1$ e $\phi>0$. Dessa forma, a esperança e a variância de y são reescritas como

$$
\mathrm{E}(\mathrm{y})=\mu \quad \mathrm{e} \quad \operatorname{Var}(\mathrm{y})=\frac{v(\mu)}{1+\phi}
$$

com $v(\mu)=\mu(1-\mu)$. Logo, temos que $\mu$ é a média da variável aleatória y e $\phi$ pode ser interpretado como parâmetro de precisão. Ou seja, para um valor fixo de $\mu$, quanto maior o valor de $\phi$, menor é a variância de y. Diferentes formas para a densidade descrita em (1.2) podem ser obtidas de acordo com a escolha dos valores para $\mu$ e $\phi$. Estas formas podem ser verificadas em Ferrari e Cribari-Neto (2004).

O logaritmo da função de verossimilhança baseado numa única observação é dado por

$$
\begin{aligned}
\ell(\mu, \phi)= & \log p(\mathrm{y} ; \mu, \phi)= \\
= & \log \Gamma(\phi)-\log \Gamma(\mu \phi)-\log \Gamma((1-\mu) \phi)+ \\
& +(\mu \phi-1) \log \mathrm{y}+[(1-\mu) \phi-1] \log (1-\mathrm{y}) .
\end{aligned}
$$


As funções escore de $\mu$ e $\phi$ são dadas por

$$
U_{\mu}(\mu, \phi)=\frac{\partial \ell(\mu, \phi)}{\partial \mu}=\phi\left(\mathrm{y}^{*}-\mu^{*}\right)
$$

e

$$
\begin{aligned}
& U_{\phi}(\mu, \phi)=\frac{\partial \ell(\mu, \phi)}{\partial \phi}= \\
& =\mu\left(\mathrm{y}^{*}-\mu^{*}\right)+\log (1-\mathrm{y})-\psi((1-\mu) \phi)+\psi(\phi),
\end{aligned}
$$

com

$$
\mathrm{y}^{*}=\log \left(\frac{y}{1-y}\right), \quad \mu^{*}=\psi(\mu \phi)-\psi((1-\mu) \phi) \quad \text { e } \quad \psi(\lambda)=\frac{\partial \log \Gamma(\lambda)}{\partial \lambda},
$$

sendo a função $\psi(\cdot)$ conhecida como função digama.

A matriz de informação de Fisher, assumindo condições usuais de regularidade descritas em Sen e Singer (1993, Capítulo 5), é dada por

$$
K=K(\mu, \phi)=\left(\begin{array}{cc}
K_{\mu \mu} & K_{\mu \phi} \\
K_{\phi \mu} & K_{\phi \phi}
\end{array}\right)
$$

sendo

$$
\begin{aligned}
& K_{\mu \mu}=\phi^{2}\left[\psi^{\prime}(\mu \phi)+\psi^{\prime}((1-\mu) \phi)\right], \\
& K_{\phi \phi}=\mu^{2} \psi^{\prime}(\mu \phi)+(1-\mu)^{2} \psi((1-\mu) \phi)-\psi^{\prime}(\phi),
\end{aligned}
$$

e

$$
K_{\mu \phi}=K_{\phi \mu}=\phi\left[\mu \psi^{\prime}(\mu \phi)-(1-\mu) \psi^{\prime}((1-\mu) \psi)\right]
$$

sendo a função trigama $\psi^{\prime}(\cdot)$ avaliada no ponto $\lambda$ dada por

$$
\psi^{\prime}(\lambda)=\frac{\mathrm{d} \psi(\lambda)}{\mathrm{d} \lambda}=\frac{\mathrm{d}^{2} \log \Gamma(\lambda)}{\mathrm{d} \lambda^{2}}=\frac{\Gamma^{\prime \prime}(\lambda) \Gamma(\lambda)-\Gamma^{\prime}(\lambda)^{2}}{\Gamma(\lambda)^{2}} .
$$

Notemos que os parâmetros $\mu$ e $\phi$ não são ortogonais, já que $K_{\mu \phi}=K_{\phi \mu}$ é diferente de zero.

Sejam $\mathrm{y}_{1}, \ldots, \mathrm{y}_{n}$ variáveis aleatórias independentes, em que cada $\mathrm{y}_{i}, i=1, \ldots, n$, segue a densidade descrita em (1.2) com média $\mu$ e parâmetro de precisão $\phi$. Sob condições gerais de regularidade (Sen e Singer, 1993), quando o tamanho da amostra é grande, temos o seguinte resultado

$$
\left(\begin{array}{c}
\sqrt{n}(\hat{\mu}-\mu) \\
\sqrt{n}(\hat{\phi}-\phi)
\end{array}\right) \stackrel{\mathcal{D}}{\longrightarrow} \mathcal{N}_{2}\left(\mathbf{0} ; \quad K^{-1}\right)
$$


sendo $\hat{\mu}$ e $\hat{\phi}$ os estimadores de máxima verossimilhança de $\mu$ e $\phi$, respectivamente, e $K^{-1}$ a matriz inversa de $K$.

Para que os resultados inferenciais da distribuição beta propostos por Ferrari e CribariNeto (2004) fossem similares aos dos modelos lineares generalizados (McCullagh e Nelder, 1989), a função escore descrita em (1.4) fica descrita em função de uma nova resposta, $\mathrm{y}^{*}$, e de um novo parâmetro, $\mu^{*}$. Assim, considerando os resultados acima e assumindo condições usuais de regularidade, obtemos que a esperança e a variância de $\mathrm{y}^{*}$ são dadas por:

$$
\mathrm{E}\left(\frac{\partial \ell(\mu, \phi)}{\partial \mu}\right)=0 \quad \Leftrightarrow \quad \mathrm{E}\left[\phi\left(\mathrm{y}^{*}-\mu^{*}\right)\right]=0 \quad \Leftrightarrow \quad \mathrm{E}\left(\mathrm{y}^{*}\right)=\mu^{*}
$$

$\mathrm{e}$

$$
\begin{aligned}
\operatorname{Var}\left(\mathrm{y}^{*}\right) & =\operatorname{Var}\left(\mathrm{y}^{*}-\mu^{*}\right)=\mathrm{E}\left(\left[\mathrm{y}^{*}-\mu^{*}\right]^{2}\right)=\phi^{-2} \mathrm{E}\left(\phi^{2}\left[\mathrm{y}^{*}-\mu^{*}\right]^{2}\right)= \\
& =\psi^{\prime}(\mu \phi)+\psi^{\prime}((1-\mu) \phi) .
\end{aligned}
$$

As equações (1.5) e (1.6) terão utilidade no Capítulo 2 para construção das equações de estimação para modelos de regressão beta com medidas repetidas.

\subsection{Distribuição Simplex}

Nesta seção, apresentamos a distribuição simplex padrão a qual faz parte de uma classe de modelos proposta por Barndorff-Nielsen e Jorgensen (1991) que assumem valores pertencentes ao intervalo unitário $(0,1)$. Para essa distribuição, podemos utilizar resultados desenvolvidos para a família dos modelos de dispersão (J $\phi$ rgensen, 1997), que é composta pelas distribuições com densidade (ou função de probabilidade) da variável aleatória y escritas na forma

$$
p(\mathrm{y} ; \mu, \phi)=\exp \left\{c(y ; \phi)-\frac{\phi}{2} d(\mathrm{y} ; \mu)\right\}
$$

com y $\in \mathcal{R}, \mu \in \Theta \subseteq \mathcal{R}, \phi>0$ e $c$ e $d$ funções duplamente diferenciáveis. O desvio unitário $d(\mathrm{y} ; \mu)$ deve ser não negativo, assumindo o valor zero se e somente se $\mathrm{y}=\mu$. A função de variância unitária é dada por

$$
v(\mu)=2\left(\frac{\partial^{2} d}{\partial \mu^{2}}(\mu ; \mu)\right)^{-1},
$$

que assumimos ser finita e positiva.

A distribuição simplex padrão ou, simplesmente, distribuição simplex com parâmetros $\mu \in(0,1)$ e $\phi>0, \mathcal{S}^{-}(\mu, \phi)$, tem função densidade de probabilidade da variável aleatória 
y escrita na forma (1.7), com $0<\mathrm{y}<1$, sendo

$$
c(y ; \phi)=-\frac{1}{2} \log \left\{2 \pi \phi^{-1}[\mathrm{y}(1-\mathrm{y})]^{3}\right\} \quad \text { e } \quad d(\mathrm{y} ; \mu)=\frac{(\mathrm{y}-\mu)^{2}}{\mathrm{y}(1-\mathrm{y}) \mu^{2}(1-\mu)^{2}} .
$$

A função de variância unitária da distribuição simplex é dada por $v(\mu)=\mu^{3}(1-\mu)^{3}$ e a esperança e a variância de y são dadas por

$$
\mathrm{E}(\mathrm{y})=\mu
$$

e

$$
\operatorname{Var}(\mathrm{y})=\mu(1-\mu)-\frac{1}{\sqrt{2 \phi^{-1}}} \exp \left(\frac{\phi}{\mu^{2}(1-\mu)^{2}}\right) \Gamma\left(\frac{1}{2}, \frac{\phi}{2 \mu^{2}(1-\mu)^{2}}\right)
$$

sendo a função gama incompleta $\Gamma(\cdot, \cdot)$ avaliada nos pontos $a$ e $b$ definida por $\Gamma(a, b)=$ $\int_{b}^{\infty} \mathrm{y}^{a-1} e^{-\mathrm{y}} d \mathrm{y}$.

Em modelos de dispersão (J $\phi$ rgensen, 1997), a função desvio unitário tem seu papel do ponto de vista estatístico similar à função desvio em modelos lineares generalizados (McCullagh e Nelder, 1989) ou à soma de quadrados dos resíduos em análise de variância (Neter et al., 1996). No caso específico da distribuição simplex, Song e Tan (2000) mostram que

$$
\mathrm{E}[d(\mathrm{y} ; \mu)]=\phi^{-1} .
$$

Logo, pelas equações (1.8) e (1.10), temos que $\mu$ é a média da variável resposta y e $\phi^{-1}$ pode ser interpretado como parâmetro de dispersão.

O logaritmo da função de verossimilhança baseada em apenas uma observação é

$$
\ell(\mu, \phi)=\log p(\mathrm{y} ; \mu, \phi)=c(\mathrm{y} ; \phi)-\frac{\phi}{2} d(\mathrm{y} ; \mu)
$$

A função escore é obtida derivando o logaritmo da função de verossimilhança com respeito aos parâmetros desconhecidos, ou seja, $\mu$ e $\phi$. Assim, temos

e

$$
U_{\mu}(\mu, \phi)=\frac{\partial \ell(\mu, \phi)}{\partial \mu}=-\frac{\phi}{2} \frac{\partial d(\mathrm{y} ; \mu)}{\partial \mu}
$$

$$
U_{\phi}(\mu, \phi)=\frac{\partial \ell(\mu, \phi)}{\partial \phi}=\frac{\partial c(\mathrm{y} ; \phi)}{\partial \phi}-\frac{1}{2} d(\mathrm{y} ; \mu)
$$

Notemos que, assumindo condições usuais de regularidade (Sen e Singer, 1993, Capítulo 5), obtemos os seguintes resultados para os valores esperados das funções escore:

e

$$
\mathrm{E}\left(\frac{\partial \ell(\mu, \phi)}{\partial \mu}\right)=0 \Leftrightarrow \mathrm{E}\left(-\frac{1}{2} \frac{\partial d(\mathrm{y} ; \mu)}{\partial \mu}\right)=0
$$

$$
\mathrm{E}\left(\frac{\partial \ell(\mu, \phi)}{\partial \phi}\right)=0 \Leftrightarrow \mathrm{E}\left(\frac{\partial c(\mathrm{y} ; \phi)}{\partial \phi}-\frac{1}{2} d(\mathrm{y} ; \mu)\right)=0
$$


A matriz de informação de Fisher, também considerando condições usuais de regularidade, é dada por

$$
K=K(\mu, \phi)=\left(\begin{array}{cc}
K_{\mu \mu} & K_{\mu \phi} \\
K_{\phi \mu} & K_{\phi \phi}
\end{array}\right)
$$

sendo

$$
\begin{aligned}
& K_{\mu \mu}=\mathrm{E}\left(\left[\frac{\partial \ell(\mu, \phi)}{\partial \mu}\right]^{2}\right)=-\mathrm{E}\left(\frac{\partial^{2} \ell(\mu, \phi)}{\partial \mu^{2}}\right)=\frac{\phi}{2} \mathrm{E}\left(\frac{\partial^{2} d(\mathrm{y} ; \mu)}{\partial \mu^{2}}\right), \\
& K_{\phi \phi}=\mathrm{E}\left(\left[\frac{\partial \ell(\mu, \phi)}{\partial \phi}\right]^{2}\right)=-\mathrm{E}\left(\frac{\partial^{2} \ell(\mu, \phi)}{\partial \phi^{2}}\right)=-\mathrm{E}\left(\frac{\partial^{2} c(\mathrm{y} ; \phi)}{\partial \phi^{2}}\right),
\end{aligned}
$$

$\mathrm{e}$

$$
\begin{aligned}
K_{\mu \phi}=K_{\phi \mu} & =\mathrm{E}\left(\frac{\partial \ell(\mu, \phi)}{\partial \mu} \frac{\partial \ell(\mu, \phi)}{\partial \phi}\right)=-\mathrm{E}\left(\frac{\partial^{2} \ell(\mu, \phi)}{\partial \mu \partial \phi}\right)=-\mathrm{E}\left(\frac{\partial^{2} \ell(\mu, \phi)}{\partial \phi \partial \mu}\right)= \\
& =\frac{1}{2} \mathrm{E}\left(\frac{\partial d(\mathrm{y} ; \mu)}{\partial \mu}\right)=0 .
\end{aligned}
$$

Notemos que os parâmetros $\mu$ e $\phi$ são ortogonais, já que $K_{\mu \phi}=K_{\phi \mu}$ é igual a zero.

Sejam $\mathrm{y}_{1}, \ldots, \mathrm{y}_{n}$ variáveis aleatórias independentes, em que cada $\mathrm{y}_{i}, i=1, \ldots, n$, segue a densidade descrita em (1.7) com média $\mu$ e parâmetro de dispersão $\phi^{-1}$. Sob condições gerais de regularidade (Sen e Singer, 1993), quando o tamanho da amostra é grande, temos o seguinte resultado:

$$
\left(\begin{array}{c}
\sqrt{n}(\hat{\mu}-\mu) \\
\sqrt{n}(\hat{\phi}-\phi)
\end{array}\right) \stackrel{\mathcal{D}}{\longrightarrow} \mathcal{N}_{2}\left(\mathbf{0} ; K^{-1}\right)
$$

sendo $\hat{\mu}$ e $\hat{\phi}$ os estimadores de máxima verossimilhança de $\mu$ e $\phi$, respectivamente, e $K^{-1}$ a matriz inversa de $K$.

A seguir, apresentamos algumas propriedades da distribuição simplex, provadas em Song e Tan (2000) ou em Song et al. (2004), que serão utilizadas no Capítulo 3. 


\section{Proposição 1.1.}

(1) $\quad E[(y-\mu) d(y ; \mu)]=0$

(2) $E\left[(y-\mu) \frac{\partial d(y ; \mu)}{\partial \mu}\right]=-2 \phi^{-1}$;

(3) $\frac{1}{2} E\left[\frac{\partial^{2} d(y ; \mu)}{\partial \mu^{2}}\right]=\frac{3 \phi^{-1}}{\mu(1-\mu)}+\frac{1}{\mu^{3}(1-\mu)^{3}}$;

(4) $E\left[d^{2}(y ; \mu)\right]=3\left(\phi^{-1}\right)^{2} \Rightarrow \operatorname{Var}[d(y ; \mu)]=2\left(\phi^{-1}\right)^{2}$;

(5) $u(y ; \mu)=-\frac{1}{2} \frac{\partial d(y ; \mu)}{\partial \mu}=\frac{y-\mu}{\mu(1-\mu)}\left\{d(y ; \mu)+\frac{1}{\mu^{2}(1-\mu)^{2}}\right\}$;

(6) $\operatorname{Var}[u(y ; \mu)]=\frac{\phi^{-1}}{2} E\left[\frac{\partial^{2} d(y ; \mu)}{\partial \mu^{2}}\right]$.

\subsection{Funções de estimação}

Nesta seção, introduzimos alguns conceitos sobre funções de estimação baseados em Artes e Botter (2005) e J $\phi$ rgensen e Labouriau (1994).

Uma função de estimação é uma função dos dados (y) e dos parâmetros de interesse $(\boldsymbol{\theta})$. Um ponto importante no estudo dessas funções é o estabelecimento de condições que garantam que os estimadores dos parâmetros envolvidos possuam boas propriedades. Em geral, desejamos que os estimadores sejam consistentes e tenham distribuição assintótica conhecida.

Seja $(\mathcal{X}, \mathcal{A}, \mathcal{P})$ um espaço de probabilidade, com $\mathcal{X} \in \mathbf{R}$ e $\mathcal{P}=\left\{P_{\theta}: \boldsymbol{\theta} \in \Theta \subseteq \mathbf{R}^{p}\right\}$, para algum $p \in \mathbf{N}$ ( $p$ é um valor fixado referente à dimensão do espaço paramétrico $\Theta$ ). Por definição, uma função $\psi: \mathcal{X} \times \Theta \rightarrow \mathbf{R}^{p}$, é uma função de estimação se para cada $\boldsymbol{\theta} \in \Theta, \boldsymbol{\psi}(. ; \boldsymbol{\theta})=\left(\psi_{1}, \ldots, \psi_{p}\right)^{\top}$ é uma variável aleatória.

Assumindo a existência de uma amostra de $n$ vetores aleatórios independentes $\mathbf{y}_{i}=$ $\left(\mathrm{y}_{i 1}, \ldots, \mathrm{y}_{i t_{i}}\right)^{\top}, i=1, \ldots, n$, e que a cada vetor esteja associada uma função de estimação $\boldsymbol{\psi}_{i}$, estendemos o conceito de função de estimação para a amostra por

$$
\boldsymbol{\Psi}_{n}(\mathbf{y} ; \boldsymbol{\theta})=\sum_{i=1}^{n} \boldsymbol{\psi}_{i}\left(\mathbf{y}_{i} ; \boldsymbol{\theta}\right)
$$

com dimensão $(p \times 1)$, sendo $\mathbf{y}=\left(\mathbf{y}_{1}^{\top}, \ldots, \mathbf{y}_{n}^{\top}\right)^{\top}$ um vetor $(N \times 1), N=\sum_{i=1}^{n} t_{i}$.

O estudo das funções de estimação que apresentamos a seguir, restringe-se àquelas cujas raízes são estimadores dos parâmetros de interesse, ou seja:

$$
\boldsymbol{\Psi}_{n}\left(\mathbf{y} ; \hat{\boldsymbol{\theta}}_{n}\right)=\mathbf{0}
$$


a qual é denominada equação de estimação.

Para simplificar a notação, apresentamos algumas propriedades de funções de estimação denotando $\boldsymbol{\Psi}_{n}(\mathbf{y} ; \boldsymbol{\theta})$ por $\boldsymbol{\Psi}_{n}(\boldsymbol{\theta})$, sempre que isso não prejudicar a clareza do texto.

As funções de estimação $\boldsymbol{\Psi}_{n}(\boldsymbol{\theta})$ e $\boldsymbol{\Phi}_{n}(\boldsymbol{\theta})$ são funções de estimação equivalentes se

$$
\boldsymbol{\Psi}_{n}(\boldsymbol{\theta})=\mathbf{C}(\boldsymbol{\theta}) \boldsymbol{\Phi}_{n}(\boldsymbol{\theta})
$$

sendo $\mathbf{C}(\boldsymbol{\theta})$ uma matriz quadrada não singular e não estocástica. Isto é, $\boldsymbol{\Psi}_{n}(\boldsymbol{\theta})$ e $\boldsymbol{\Phi}_{n}(\boldsymbol{\theta})$ têm as mesmas raízes.

A função de estimação $\boldsymbol{\Psi}_{n}(\boldsymbol{\theta})$ é uma função de estimação não viciada se

$$
\mathrm{E}_{\theta}\left(\boldsymbol{\Psi}_{n}(\boldsymbol{\theta})\right)=\mathbf{0}
$$

Se todas as funções de estimação $\boldsymbol{\psi}_{i}, i=1, \ldots, n$, são não viciadas, então a função de estimação $\Psi_{n}$ baseada na amostra de tamanho $n$ também será não viciada.

A matriz de variabilidade de uma função de estimação não viciada é dada por

$$
\mathbf{V}_{\Psi}(\boldsymbol{\theta})=\mathrm{E}_{\theta}\left(\boldsymbol{\Psi}_{n}(\boldsymbol{\theta}) \Psi_{n}^{\top}(\boldsymbol{\theta})\right)
$$

e a matriz de sensibilidade de uma função de estimação não viciada é dada por

$$
\mathbf{S}_{\Psi}(\boldsymbol{\theta})=\mathrm{E}_{\theta}\left(\frac{\partial}{\partial \boldsymbol{\theta}^{\top}} \boldsymbol{\Psi}_{n}(\boldsymbol{\theta})\right)
$$

sendo que ambas têm dimensão $(p \times p)$.

Uma função de estimação $\boldsymbol{\psi}=\left(\psi_{1}, \ldots, \psi_{p}\right)^{\top}: \mathcal{X} \times \Theta \rightarrow \mathbf{R}^{p}$ é dita uma função de estimação regular quando as seguintes condições são satisfeitas para todo $\boldsymbol{\theta} \in \Theta$ :

i. a função de estimação $\boldsymbol{\psi}(\mathbf{y} ; \boldsymbol{\theta})$ é não viciada;

ii. a derivada parcial de $\boldsymbol{\psi}(\mathbf{y} ; \boldsymbol{\theta})$ com respeito a $\boldsymbol{\theta}$ existe quase certamente para todo $\mathbf{y} \in \mathcal{X}$

iii. é possível permutar o sinal de integração e diferenciação da seguinte forma:

$$
\frac{\partial}{\partial \theta_{l}} \int_{\mathcal{X}} \boldsymbol{\psi}(\mathbf{y} ; \boldsymbol{\theta}) d P_{\theta}=\int_{\mathcal{X}} \frac{\partial}{\partial \theta_{l}}[\boldsymbol{\psi}(\mathbf{y} ; \boldsymbol{\theta})] d P_{\theta}
$$

$\operatorname{com} l=1, \ldots, p$.

iv. $\mathrm{E}_{\theta}\left(\psi_{j}(\boldsymbol{\theta}) \psi_{k}(\boldsymbol{\theta})\right) \in \mathbf{R}, \operatorname{com} j, k=1, \ldots, p$ e $\mathbf{V}_{\psi}(\boldsymbol{\theta})$ é positiva definida; 
v. $\mathrm{E}_{\theta}\left(\frac{\partial}{\partial \theta_{l}} \psi_{j}(\boldsymbol{\theta}) \frac{\partial}{\partial \theta_{m}} \psi_{k}(\boldsymbol{\theta})\right) \in \mathbf{R}, \operatorname{com} j, k, l, m=1, \ldots, p$ e $\mathbf{S}_{\psi}(\boldsymbol{\theta})$ é não singular.

Seja $\boldsymbol{\Psi}_{n}(\boldsymbol{\theta})$ uma função de estimação regular. Definimos a matriz de informação de Godambe de $\boldsymbol{\theta}$ associada a $\boldsymbol{\Psi}_{n}$ por

$$
\mathbf{J}_{\Psi}(\boldsymbol{\theta})=\mathbf{S}_{\Psi}(\boldsymbol{\theta})^{\top} \mathbf{V}_{\Psi}^{-1}(\boldsymbol{\theta}) \mathbf{S}_{\Psi}(\boldsymbol{\theta}),
$$

com dimensão $(p \times p)$.

A matriz de informação de Godambe $\mathbf{J}_{\Psi}(\boldsymbol{\theta})$ é igual à matriz de informação de Fisher se e somente se $\boldsymbol{\Psi}_{n}(\boldsymbol{\theta})$ é equivalente à função escore. Por exemplo, se $\boldsymbol{\Psi}_{n}(\boldsymbol{\theta})$ é a função escore e, portanto, satisfaz as condições de ser uma função de estimação regular, então $\mathbf{S}_{\Psi}(\boldsymbol{\theta})=$ $-\mathbf{V}_{\Psi}(\boldsymbol{\theta})$, fazendo com que sua matriz de informação de Godambe coincida com a matriz de informação de Fisher. De maneira geral, para todas as funções de estimação regulares que não sejam necessariamente funções escores, a informação de Godambe desempenha o papel da informação de Fisher.

Um conceito importante desenvolvido por Godambe (1960) é o de otimalidade de uma função de estimação regular. No caso de $\theta$ ser unidimensional, podemos definir uma função de estimação ótima como aquela cujas raízes possuem variância assintótica mínima. Esse conceito pode ser estendido para o caso multidimensional por meio da introdução de alguma ordenação das matrizes de covariâncias assintóticas (Chandrasekar e Kale, 1984).

Crowder (1987) estuda uma classe particular de funções de estimação que passaremos a chamar de funções de estimação lineares.

Sejam $\mathbf{Q}_{i}(\boldsymbol{\theta}), i=1, \ldots, n$, matrizes não estocásticas, não singulares e de pesos que, eventualmente, podem ser função de $\boldsymbol{\theta}$. Sejam $\mathbf{b}_{i}=\mathbf{b}_{i}\left(\mathbf{y}_{i} ; \boldsymbol{\theta}\right), i=1, \ldots, n$, vetores com média zero mutuamente independentes, satisfazendo as mesmas condições das funções de estimação regulares. A classe das funções de estimação lineares geradas por $\mathbf{b}_{i}$ é definida por

$$
\mathcal{L}(\mathbf{b})=\left\{\boldsymbol{\Psi}_{n}(\boldsymbol{\theta}) \in \mathcal{R}: \Psi_{n}(\boldsymbol{\theta})=\sum_{i=1}^{n} \mathbf{Q}_{i}(\boldsymbol{\theta}) \mathbf{b}_{i}\left(\mathbf{y}_{i} ; \boldsymbol{\theta}\right)\right\}
$$

sendo que $\mathcal{R}$ contém todas as funções regulares de $\boldsymbol{\theta}$ e $\mathbf{b}=\left(\mathbf{b}_{1}^{\top}, \ldots, \mathbf{b}_{n}^{\top}\right)^{\top}$.

O autor mostra que a função de estimação ótima dentre as da classe $\mathcal{L}(\mathbf{b})$ é dada por

$$
\Psi_{n}^{o}(\boldsymbol{\theta})=\sum_{i=1}^{n} \mathbf{Q}_{i}^{o}(\boldsymbol{\theta}) \mathbf{b}_{i}\left(\mathbf{y}_{i} ; \boldsymbol{\theta}\right)
$$


em que

$$
\mathbf{Q}_{i}^{o}(\boldsymbol{\theta})=\mathrm{E}\left(\frac{\partial \mathbf{b}_{i}}{\partial \boldsymbol{\theta}^{\top}}\right)^{\top} \operatorname{Cov}\left(\mathbf{b}_{i}\right)^{-1}
$$

com

$$
\operatorname{Cov}\left(\mathbf{b}_{i}\right)=\operatorname{Var}\left(\mathbf{b}_{i}\right)^{1 / 2} \mathbf{R}\left(\mathbf{b}_{i}\right) \operatorname{Var}\left(\mathbf{b}_{i}\right)^{1 / 2},
$$

sendo $\operatorname{Var}\left(\mathbf{b}_{i}\right)$ uma matriz diagonal contendo $\operatorname{Var}\left(\mathrm{b}_{i j}\right)$ e $\mathbf{R}\left(\mathbf{b}_{i}\right)$ a verdadeira matriz de correlação de $\mathbf{b}_{i}$, para $i=1, \ldots, n$ e $j=1, \ldots, t$.

É importante ressaltar que se considerarmos $\mathbf{C}(\boldsymbol{\theta}) \Psi_{n}^{o}(\boldsymbol{\theta})$, sendo $\mathbf{C}(\boldsymbol{\theta})$ uma matriz não singular e não estocástica, essa nova função de estimação também será ótima.

O teorema descrito a seguir estabelece condições para a normalidade assintótica de estimadores obtidos a partir de funções de estimação regulares. Esse teorema trata do caso em que $\boldsymbol{\theta}$ é multidimensional e, além disso, os vetores resposta são mutuamente independentes, mas não identicamente distribuídos.

Teorema 1. Considerando-se

a. $\boldsymbol{y}_{i}, i=1,2, \ldots, n$, vetores aleatórios t-dimensionais independentes;

b. $\boldsymbol{\psi}_{i}(\boldsymbol{\theta})=\left(\psi_{i 1}, \ldots, \psi_{i p}\right)^{\top}, i=1,2, \ldots, n$, funções de estimação regulares;

c. $\boldsymbol{\Psi}_{n}(\boldsymbol{\theta})=\sum_{i=1}^{n} \boldsymbol{\psi}_{i}(\boldsymbol{\theta})$;

d. $\operatorname{para} \delta>0$,

$$
\begin{aligned}
E_{\theta}\left\{\sup _{\boldsymbol{h}:\|\boldsymbol{h}\| \leq \delta}\left\|\frac{\partial}{\partial \boldsymbol{\theta}} \boldsymbol{\psi}_{i}(\boldsymbol{\theta}+\boldsymbol{h})-\frac{\partial}{\partial \boldsymbol{\theta}} \boldsymbol{\psi}_{i}(\boldsymbol{\theta})\right\|\right\}=\phi_{\delta_{i}}, \\
\frac{1}{n} \sum_{i=1}^{n} \phi_{\delta_{i}} \rightarrow \phi_{\delta} \text { e } \phi_{\delta} \rightarrow 0 \text { quando } \delta \rightarrow 0
\end{aligned}
$$

e. para $\delta>0$, existe $\epsilon, 0<\epsilon<1$, tal que

$$
n^{-1-\epsilon} \sum_{i=1}^{n} E_{\theta}\left\{\sup _{\boldsymbol{h}:\|\boldsymbol{h}\| \leq \delta}\left\|\frac{\partial}{\partial \boldsymbol{\theta}} \boldsymbol{\psi}_{i}(\boldsymbol{\theta}+\boldsymbol{h})-\frac{\partial}{\partial \boldsymbol{\theta}} \boldsymbol{\psi}_{i}(\boldsymbol{\theta})-\phi_{\delta_{i}}\right\|\right\}^{1+\epsilon} \rightarrow \mathbf{0}
$$

f. que para $\dot{\boldsymbol{\psi}}_{i}(\boldsymbol{\theta})=\frac{\partial}{\partial \boldsymbol{\theta}^{\top}} \boldsymbol{\psi}_{i}(\boldsymbol{\theta})$,

$$
\frac{1}{n} \sum_{i=1}^{n} E\left\{\dot{\boldsymbol{\psi}}_{i}(\boldsymbol{\theta})\right\}=\frac{1}{n} \sum_{i=1}^{n} \boldsymbol{S}_{i}(\boldsymbol{\theta}) \rightarrow \boldsymbol{S}(\boldsymbol{\theta}),
$$

com $\boldsymbol{S}$ não singular e $\boldsymbol{S}_{i}=E\left\{\dot{\boldsymbol{\psi}}_{i}(\boldsymbol{\theta})\right\}$; 
g. que existe $\epsilon, 0<\epsilon<1$, tal que

$$
n^{-1-\epsilon} \sum_{i=1}^{n} E\left|\dot{\boldsymbol{\psi}}_{i}(\boldsymbol{\theta})-\boldsymbol{S}_{i}(\boldsymbol{\theta})\right|^{1+\epsilon} \rightarrow \mathbf{0}
$$

h. que existe $\epsilon, 0<\epsilon<1$, tal que $\max _{1 \leq i \leq n} \max _{1 \leq j \leq t} E\left|\psi_{i j}\right|^{2+\epsilon}<\infty$;

i. $n^{-1} \sum_{i=1}^{n} \operatorname{Cov}\left(\boldsymbol{\psi}_{i}\right) \rightarrow \boldsymbol{V}(\boldsymbol{\theta})$, positiva definida;

j. $\hat{\boldsymbol{\theta}}_{n}$ como a solução de $\boldsymbol{\Psi}(\boldsymbol{w})=\mathbf{0}, \boldsymbol{w} \in \Theta$,

e sob condições que garantam a existência de uma seqüência de raízes de $\boldsymbol{\Psi}_{n}(\boldsymbol{w})$ que seja limitada em probabilidade ou restrita a um conjunto compacto q.c. quando $n$ tende a infinito, vem que

$$
\hat{\boldsymbol{\theta}}_{n} \stackrel{\mathcal{P}}{\longrightarrow} \boldsymbol{\theta} .
$$

Sob essas hipóteses,

$$
\sqrt{n}\left(\hat{\boldsymbol{\theta}}_{n}-\boldsymbol{\theta}\right) \stackrel{\mathcal{D}}{\longrightarrow} \mathrm{N}_{p}\left(\mathbf{0}, \boldsymbol{J}_{\Psi}^{-1}(\boldsymbol{\theta})\right)
$$

em que

$$
\begin{aligned}
\boldsymbol{J}_{\Psi}(\boldsymbol{\theta}) & =\left\{\lim _{n \rightarrow \infty} \boldsymbol{S}_{n}^{\top}(\boldsymbol{\theta})\right\}\left\{\lim _{n \rightarrow \infty} \boldsymbol{V}_{n}(\boldsymbol{\theta})\right\}^{-1}\left\{\lim _{n \rightarrow \infty} \boldsymbol{S}_{n}(\boldsymbol{\theta})\right\}= \\
& =\left\{\lim _{n \rightarrow \infty} \boldsymbol{S}_{n}^{\top}(\boldsymbol{\theta})\right\}\left\{\lim _{n \rightarrow \infty} \boldsymbol{V}_{n}^{-1}(\boldsymbol{\theta})\right\}\left\{\lim _{n \rightarrow \infty} \boldsymbol{S}_{n}(\boldsymbol{\theta})\right\}= \\
& =\lim _{n \rightarrow \infty}\left\{\boldsymbol{S}_{n}^{\top}(\boldsymbol{\theta}) \boldsymbol{V}_{n}^{-1}(\boldsymbol{\theta}) \boldsymbol{S}_{n}(\boldsymbol{\theta})\right\}
\end{aligned}
$$

com

$$
\boldsymbol{S}_{n}(\boldsymbol{\theta})=\sum_{i=1}^{n} \frac{\boldsymbol{S}_{\psi_{i}}(\boldsymbol{\theta})}{n}, \quad \boldsymbol{S}_{\psi_{i}}(\boldsymbol{\theta})=E_{\theta}\left\{\frac{\partial}{\partial \boldsymbol{\theta}^{\top}} \boldsymbol{\psi}_{i}\left(y_{i} ; \boldsymbol{\theta}\right)\right\}
$$

$e$

$$
\boldsymbol{V}_{n}(\boldsymbol{\theta})=\sum_{i=1}^{n} \frac{\boldsymbol{V}_{\psi_{i}}(\boldsymbol{\theta})}{n}, \quad \boldsymbol{V}_{\psi_{i}}(\boldsymbol{\theta})=E_{\theta}\left\{\boldsymbol{\Psi}_{n}\left(y_{i} ; \boldsymbol{\theta}\right) \boldsymbol{\Psi}_{n}^{\top}\left(y_{i} ; \boldsymbol{\theta}\right)\right\}
$$

desempenha o papel de uma matriz de informação de Godambe assintótica associada a $\Psi_{n}$.

A prova e comentários sobre cada item do Teorema 1 estão descritos em Artes (1997, p.30). 


\section{Parte I}

\section{Equações de Estimação Generalizadas}




\section{Capítulo 2}

\section{Equações de estimação para modelos de regressão beta com medidas repetidas}

Neste capítulo, propomos equações de estimação generalizadas para situações em que a variável dependente é medida de forma contínua no intervalo unitário $(0,1)$ e há mais do que uma observação realizada numa mesma unidade experimental. Essas equações de estimação são baseadas na suposição de que a resposta tem distribuição marginal beta utilizando a parametrização indexada pela média e pelo parâmetro de precisão conforme Seção 1.1 .

A seguir, apresentamos a modelagem da média sob a suposição de homogeneidade do parâmetro de precisão. Na Seção 2.2, a média e a precisão são modeladas conjuntamente via equações de estimação, com o intuito de incorporar ao modelo uma possível heterogeneidade da precisão.

\subsection{Modelagem do parâmetro de posição}

Seja $\mathbf{y}_{i}=\left(\mathrm{y}_{i 1}, \mathrm{y}_{i 2}, \ldots, \mathrm{y}_{i t_{i}}\right)^{\top}$ o vetor de respostas da $i$-ésima unidade experimental, com $i=1, \ldots, n$. Vamos assumir que a densidade marginal da variável resposta tenha distribuição beta, $\mathrm{y}_{i j} \sim \mathcal{B}\left(\mu_{i j}, \phi\right)$, dada por

$$
p\left(\mathrm{y}_{i j} ; \mu_{i j}, \phi\right)=\frac{\Gamma(\phi)}{\Gamma\left(\mu_{i j} \phi\right) \Gamma\left(\left(1-\mu_{i j}\right) \phi\right)} \mathrm{y}_{i j}^{\mu_{i j} \phi-1}\left(1-\mathrm{y}_{i j}\right)^{\left(1-\mu_{i j}\right) \phi-1} .
$$

A princípio, vamos supor que o parâmetro de precisão $\phi$ é conhecido e igual para todas as observações. Para facilitar a notação, assumimos, sem perda de generalidade, que $t_{i}=t$, $i=1, \ldots, n$. 
Consideremos que as médias $\mu_{i j}$ 's sejam modeladas por

$$
g\left(\mu_{i j}\right)=\eta_{i j}=\mathbf{x}_{i j}^{\top} \boldsymbol{\beta}
$$

sendo $g($.$) uma função monótona e duplamente diferenciável, denominada função de liga-$ ção, $\eta_{i j}$ o preditor linear, $\mathbf{x}_{i j}$ um vetor de covariáveis referente à $j$-ésima observação da $i$-ésima unidade experimental, com $i=1, \ldots, n$ e $j=1, \ldots, t$, e $\boldsymbol{\beta}=\left(\beta_{1}, \ldots, \beta_{p}\right)^{\top}, p<n$, um vetor de parâmetros desconhecidos a serem estimados.

Para construção das equações de estimação para modelos de regressão beta com medidas repetidas, utilizamos a definição de função de estimação linear ótima descrita em (1.14). Nessa definição, é necessário ter vetores $\mathbf{b}_{i}=\mathbf{b}_{i}\left(\mathbf{y}_{i} ; \boldsymbol{\beta}\right), i=1, \ldots, n$, com média zero, mutuamente independentes e satisfazendo as mesmas propriedades das funções de estimação regulares (veja definição na Seção 1.3).

No contexto de modelos de regressão beta com medidas repetidas, inicialmente, definimos $\mathbf{b}_{i}=\mathbf{y}_{i}-\boldsymbol{\mu}_{i}$, com $\mathbf{y}_{i}=\left(\mathrm{y}_{i 1}, \ldots, \mathrm{y}_{i t}\right)^{\top}$ e $\boldsymbol{\mu}_{i}=\left(\mu_{i 1}, \ldots, \mu_{i t}\right)^{\top}, i=1, \ldots, n$, os quais satisfazem tais propriedades. Entretanto, quando temos independência entre as observações da mesma unidade experimental, esses vetores $\mathbf{b}_{i}$ 's não geram uma classe $\mathcal{L}(\mathbf{b})$ (veja definição em (1.13)) contendo a função escore apresentada por Ferrari e Cribari-Neto (2004). Para contornarmos esse problema e desenvolvermos equações de estimação que generalizam tanto o caso em que há correlação entre as observações da mesma unidade experimental como o caso mais simples em que há independência entre elas, propomos

$$
\mathbf{b}_{i}=\mathbf{y}_{i}^{*}-\boldsymbol{\mu}_{i}^{*}
$$

$\operatorname{com} \mathbf{y}_{i}^{*}=\left(\mathrm{y}_{i 1}^{*}, \ldots, \mathrm{y}_{i t}^{*}\right)^{\top}$ e $\boldsymbol{\mu}_{i}^{*}=\left(\mu_{i 1}^{*}, \ldots, \mu_{i t}^{*}\right)^{\top}, i=1, \ldots, n$. Esses vetores geram uma classe $\mathcal{L}(\mathbf{b})$ que, no caso de independência entre todas as observações, contém a função escore descrita por Ferrari e Cribari e, também, são vetores com média zero (veja resultado apresentado na equação (1.5)), mutuamente independentes e com as propridades das funções de estimação regulares.

Assim, construímos as equações de estimação para modelos de regressão beta com medidas repetidas, considerando a densidade marginal de $\mathrm{y}_{i j}$ e o componente sistemático descritos, respectivamente, nas equações (2.1) e (2.2) e definindo $\mathbf{b}_{i}=\mathbf{y}_{i}^{*}-\boldsymbol{\mu}_{i}^{*}, i=1, \ldots, n$. Assumindo dependência entre as observações da mesma unidade experimental $i$, os termos da função de estimação definidos em (1.14) são dados por

e

$$
\mathrm{E}\left(\frac{\partial \mathbf{b}_{i}}{\partial \boldsymbol{\beta}^{\top}}\right)^{\top}=-\mathbf{X}_{i}^{\top} \boldsymbol{\Lambda}_{i}
$$

$$
\operatorname{Cov}\left(\mathbf{b}_{i}\right)=\operatorname{Var}\left(\mathbf{y}_{i}^{*}\right)^{1 / 2} \mathbf{R}\left(\mathbf{y}_{i}^{*}\right) \operatorname{Var}\left(\mathbf{y}_{i}^{*}\right)^{1 / 2}=\mathbf{A}_{i}^{1 / 2} \mathbf{R}\left(\mathbf{y}_{i}^{*}\right) \mathbf{A}_{i}^{1 / 2}
$$


sendo $\mathbf{X}_{i}=\left(\mathbf{x}_{i 1}, \ldots, \mathbf{x}_{i t}\right)^{\top}, \quad \boldsymbol{\Lambda}_{i}=\phi \mathbf{G}_{i} \mathbf{A}_{i}, \quad \mathbf{A}_{i}=\operatorname{diag}\left(\mathrm{a}_{i 1}, \ldots, \mathrm{a}_{i t}\right)$ e $\mathbf{R}\left(\mathbf{y}_{i}^{*}\right)$ a verdadeira matriz de correlação de $\mathbf{y}_{i}^{*}, \operatorname{com} \mathbf{G}_{i}=\operatorname{diag}\left(\partial g^{-1}\left(\eta_{i 1}\right) / \partial \eta_{i 1}, \ldots, \partial g^{-1}\left(\eta_{i t}\right) / \partial \eta_{i t}\right)$ e $\mathbf{a}_{i j}=$ $\psi^{\prime}\left(\mu_{i j} \phi\right)+\psi^{\prime}\left(\left(1-\mu_{i j}\right) \phi\right), i=1, \ldots, n$ e $j=1, \ldots, t$. Notemos que a $a_{i j}$ é a variância de $\mathrm{y}_{i j}^{*}$ conforme definição em (1.6) e que $\boldsymbol{\Lambda}_{i}$ é uma matriz simétrica. As contas detalhadas estão apresentadas na Seção A.1.1 do Apêndice A e as possíveis expressões para $\mathbf{G}$ de acordo com a função de ligação $g(\cdot)$ adotada estão apresentadas na Tabela C.1 do Apêndice C.

A função de estimação linear ótima de $\boldsymbol{\beta}$, quando consideramos a verdadeira matriz de correlação de $\mathbf{y}_{i}^{*}$ e $\phi$ conhecido, é equivalente a

$$
\boldsymbol{\Psi}_{1}^{o}(\boldsymbol{\beta})=\sum_{i=1}^{n} \mathbf{X}_{i}^{\top} \boldsymbol{\Lambda}_{i} \operatorname{Cov}\left(\mathbf{b}_{i}\right)^{-1}\left(\mathbf{y}_{i}^{*}-\boldsymbol{\mu}_{i}^{*}\right)
$$

Sob as condições do Teorema 1 , temos que $\hat{\boldsymbol{\beta}}$, solução de $\boldsymbol{\Psi}_{1}^{o}(\hat{\boldsymbol{\beta}})=\mathbf{0}$, é um estimador consistente de $\boldsymbol{\beta}$ e que

$$
\sqrt{n}(\hat{\boldsymbol{\beta}}-\boldsymbol{\beta}) \stackrel{\mathcal{D}}{\longrightarrow} \mathrm{N}_{p}\left(\mathbf{0}, \lim _{n \rightarrow \infty} n\left\{\sum_{i=1}^{n} \mathbf{X}_{i}^{\top} \boldsymbol{\Lambda}_{i} \operatorname{Cov}\left(\mathbf{b}_{i}\right)^{-1} \boldsymbol{\Lambda}_{i} \mathbf{X}_{i}\right\}^{-1}\right)
$$

Na prática, a verdadeira matriz de correlação é geralmente desconhecida. Seguindo a proposta de Liang e Zeger (1986), definimos $\mathbf{R}(\boldsymbol{\alpha})$ como sendo uma matriz simétrica $(t \times t)$ satisfazendo as condições para ser uma matriz de correlação, denominada matriz de correlação de trabalho em que $\boldsymbol{\alpha}$, um vetor $(s \times 1)$, caracteriza completamente $\mathbf{R}(\boldsymbol{\alpha})$. Notemos que $\mathbf{R}(\boldsymbol{\alpha})$ não precisa ser necessariamente a verdadeira matriz de correlação dos $\mathbf{y}_{i}^{*}$ s.

Com isso, a função de estimação generalizada de $\boldsymbol{\beta}$ é dada por

$$
\Psi_{1}(\boldsymbol{\beta})=\sum_{i=1}^{n} \mathbf{X}_{i}^{\top} \boldsymbol{\Lambda}_{i} \boldsymbol{\Omega}_{i}^{-1}\left(\mathbf{y}_{i}^{*}-\boldsymbol{\mu}_{i}^{*}\right)=\sum_{i=1}^{n} \mathbf{X}_{i}^{\top} \mathbf{W}_{i} \boldsymbol{\Lambda}_{i}^{-1} \mathbf{b}_{i},
$$

sendo $\boldsymbol{\Omega}_{i}=\mathbf{A}_{i}^{1 / 2} \mathbf{R}(\boldsymbol{\alpha}) \mathbf{A}_{i}^{1 / 2}$ e $\quad \mathbf{W}_{i}=\boldsymbol{\Lambda}_{i} \boldsymbol{\Omega}_{i}^{-1} \boldsymbol{\Lambda}_{i}$.

Entretanto, a função de estimação definida em (2.4) deixa de ser a ótima. Nesse caso, é necessário que um estimador $\hat{\boldsymbol{\alpha}}$ de $\boldsymbol{\alpha}$ seja determinado de modo que o estimador de $\boldsymbol{\beta}$, obtido a partir de (2.4), seja consistente e assintoticamente normal. Além disso, como $\phi$ quase sempre é desconhecido, também é necessário propor um estimador para esse parâmetro.

O próximo teorema descreve algumas condições que os estimadores de $\boldsymbol{\alpha}$ e $\phi$ devem satisfazer de modo que o estimador de $\boldsymbol{\beta}$ preserve as propriedades mencionadas acima. 
Teorema 2. Seja $\hat{\boldsymbol{\beta}}$ a raiz de (2.4). Sob condições gerais de regularidade e assumindo que

a. $\hat{\boldsymbol{\alpha}}(\boldsymbol{\beta}, \phi)$ é um estimador $\sqrt{n}$-consistente ${ }^{1}$ de $\boldsymbol{\alpha}$ dados $\boldsymbol{\beta}$ e $\phi$;

b. $\hat{\phi}(\boldsymbol{\beta})$ é um estimador $\sqrt{n}$-consistente de $\phi$ dado $\boldsymbol{\beta}$; e

c. $|\partial \hat{\boldsymbol{\alpha}}(\boldsymbol{\beta}, \phi) / \partial \phi| \leq \boldsymbol{H}(\boldsymbol{y}, \boldsymbol{\beta})$, sendo $\boldsymbol{H}(\boldsymbol{y}, \boldsymbol{\beta})$ uma função $O_{p}(1)$,

então temos que $\hat{\boldsymbol{\beta}}$ é um estimador consistente de $\boldsymbol{\beta}$ e

$$
\sqrt{n}(\hat{\boldsymbol{\beta}}-\boldsymbol{\beta}) \stackrel{\mathcal{D}}{\longrightarrow} \mathrm{N}_{p}\left(\mathbf{0}, \boldsymbol{J}^{-1}\right)
$$

com $\boldsymbol{J}=\lim _{n \rightarrow \infty} \boldsymbol{J}_{n} / n$, sendo $\boldsymbol{J}_{n}$ a matriz de informação de Godambe de $\boldsymbol{\beta}$ associada à $\Psi_{1}$ dada por

$$
\boldsymbol{J}_{n}=\left\{\sum_{i=1}^{n} \boldsymbol{S}_{i}\right\}\left\{\sum_{i=1}^{n} \boldsymbol{V}_{i}\right\}^{-1}\left\{\sum_{i=1}^{n} \boldsymbol{S}_{i}\right\}
$$

em que

$$
\sum_{i=1}^{n} \boldsymbol{S}_{i}=E\left[\frac{\partial}{\partial \boldsymbol{\beta}^{\top}} \boldsymbol{\Psi}_{1}(\boldsymbol{\beta})\right]=-\sum_{i=1}^{n} \boldsymbol{X}_{i}^{\top} \boldsymbol{W}_{i} \boldsymbol{X}_{i}
$$

$e$

$$
\sum_{i=1}^{n} \boldsymbol{V}_{i}=E\left[\boldsymbol{\Psi}_{1}(\boldsymbol{\beta}) \boldsymbol{\Psi}_{1}^{\top}(\boldsymbol{\beta})\right]=\sum_{i=1}^{n} \boldsymbol{X}_{i}^{\top} \boldsymbol{\Lambda}_{i} \boldsymbol{\Omega}_{i}^{-1} \operatorname{Cov}\left(\boldsymbol{b}_{i}\right) \boldsymbol{\Omega}_{i}^{-1} \boldsymbol{\Lambda}_{i} \boldsymbol{X}_{i}
$$

A prova desse teorema é análoga à encontrada em Artes (1997, Teorema 6, p.47), a qual também enfatiza que os resultados assintóticos desse teorema são válidos mesmo quando $\mathbf{R}(\boldsymbol{\alpha})$ não corresponde à verdadeira matriz de correlação de $\mathbf{y}_{i}^{*}$.

A matriz de covariâncias de $\hat{\boldsymbol{\beta}}$ pode ser consistentemente estimada por

$$
\hat{\mathbf{J}}_{n}^{-1}=\left\{\sum_{i=1}^{n} \hat{\mathbf{S}}_{i}\right\}^{-1}\left\{\sum_{i=1}^{n} \mathbf{X}_{i}^{\top} \hat{\mathbf{\Lambda}}_{i} \hat{\mathbf{\Omega}}_{i}^{-1} \hat{\mathbf{b}}_{i} \hat{\mathbf{b}}_{i}^{\top} \hat{\boldsymbol{\Omega}}_{i}^{-1} \hat{\boldsymbol{\Lambda}}_{i} \mathbf{X}_{i}\right\}\left\{\sum_{i=1}^{n} \hat{\mathbf{S}}_{i}\right\}^{-1} .
$$

A estimativa da expressão acima é obtida substituindo $\boldsymbol{\beta}, \boldsymbol{\alpha}$ e $\phi$ por suas respectivas estimativas consistentes. Na literatura especializada esse estimador recebe os nomes de

$$
{ }^{1}\|\hat{\boldsymbol{\alpha}}(\boldsymbol{\beta}, \phi)-\boldsymbol{\alpha}\|=O_{p}\left(n^{-1 / 2}\right)
$$


estimador robusto, empírico ou sanduíche. Quando $\mathbf{R}(\boldsymbol{\alpha})$ representar a verdadeira matriz de correlação dos $\mathbf{y}_{i}^{*}$ 's, então $\Omega_{i}=\operatorname{Cov}\left(\mathbf{b}_{i}\right)$ e o estimador robusto se reduz a

$$
\hat{\mathbf{J}}_{n}^{-1}=-\left\{\sum_{i=1}^{n} \hat{\mathbf{S}}_{i}\right\}^{-1}
$$

o qual é conhecido como estimador "naive" ou "model-based".

Considerando que o modelo de regressão está corretamente especificado, o estimador "naive" é consistente se a matriz de correlação de trabalho também está corretamente especificada. Já o estimador robusto é sempre consistente. Além disso, o estimador robusto é assintoticamente não viesado, mas pode ser altamente viesado quando o número de unidades experimentais $(n)$ for pequeno. Quando $n<20$, o estimador "naive" pode ter melhores propriedades (Prentice, 1988) mesmo se $\mathbf{R}(\boldsymbol{\alpha})$ não estiver corretamente especificada.

Uma indicação da adequação de uma matriz $\mathbf{R}(\boldsymbol{\alpha})$ ao modelo ocorre quando as duas estimativas, "naive" e robusta, são próximas (Johnston, 1996).

\subsubsection{Estimação de $\beta, \phi$ e $\alpha$}

O processo iterativo para calcular $\hat{\boldsymbol{\beta}}, \hat{\phi}$ e $\hat{\boldsymbol{\alpha}}$ combina o método scoring de Fisher para estimar $\boldsymbol{\beta}$ com o método dos momentos para estimar $\boldsymbol{\alpha}$ e $\phi$. Expandindo a EEG dada na equação (2.4) em torno de um valor inicial $\hat{\boldsymbol{\beta}}^{(0)}$, o processo iterativo para estimar $\boldsymbol{\beta}$ é dado por

$$
\begin{aligned}
\hat{\boldsymbol{\beta}}^{(m+1)} & =\hat{\boldsymbol{\beta}}^{(m)}-\left\{\mathrm{E}\left[\frac{\partial}{\partial \boldsymbol{\beta}^{\top}} \Psi_{1}\left(\hat{\boldsymbol{\beta}}^{(m)}\right)\right]\right\}^{-1} \mathbf{\Psi}_{1}\left(\hat{\boldsymbol{\beta}}^{(m)}\right)= \\
& =\hat{\boldsymbol{\beta}}^{(m)}+\left\{\left[\sum_{i=1}^{n} \mathbf{X}_{i}^{\top} \hat{\mathbf{W}}_{i} \mathbf{X}_{i}\right]^{-1}\left[\sum_{i=1}^{n} \mathbf{X}_{i}^{\top} \hat{\mathbf{W}}_{i} \hat{\boldsymbol{\Lambda}}_{i}^{-1} \hat{\mathbf{b}}_{i}\right]\right\}^{(m)}
\end{aligned}
$$

sendo $m=0,1,2, \ldots$ o número de iterações. O índice $m$ no lado direito das equações acima indica que as matrizes e os vetores são atualizados pelas estimativas de $\boldsymbol{\beta}, \boldsymbol{\alpha}$ e $\phi$ da $m$-ésima iteração.

Reescrevendo (2.5) na forma de um processo iterativo de mínimos quadrados reponderados que emprega uma matriz de pesos $\mathbf{W}_{i}$ e uma variável dependente modificada $\mathbf{z}_{i}$, temos:

$$
\hat{\boldsymbol{\beta}}^{(m+1)}=\left\{\left[\sum_{i=1}^{n} \mathbf{X}_{i}^{\top} \hat{\mathbf{W}}_{i} \mathbf{X}_{i}\right]^{-1}\left[\sum_{i=1}^{n} \mathbf{X}_{i}^{\top} \hat{\mathbf{W}}_{i} \mathbf{z}_{i}\right]\right\}^{(m)}
$$


sendo $\mathbf{z}_{i}=\hat{\boldsymbol{\eta}}_{i}+\hat{\boldsymbol{\Lambda}}_{i}^{-1} \hat{\mathbf{b}}_{i}$. O processo iterativo escrito conforme a equação (2.6) será útil, principalmente, para obtermos as medidas de diagnóstico descritas no Capítulo 5.

Utilizando o método dos momentos e a $\operatorname{Var}\left(\mathrm{y}_{i j}\right)=v\left(\mu_{i j}\right) / 1+\phi$ definida na equação (1.3), a estimativa de $\phi$ obtida no $m$-ésimo passo do processo iterativo é dada por

$$
\hat{\phi}^{(m)}=\left(\left\{\sum_{i=1}^{n} \sum_{j=1}^{t}\left(\frac{\mathrm{y}_{i j}-\hat{\mu}_{i j}^{(m)}}{\sqrt{\hat{\mu}_{i j}^{(m)}\left(1-\hat{\mu}_{i j}^{(m)}\right)}}\right)^{2} /(n t-p)\right\}^{-1}-1\right) .
$$

O procedimento da EEG para estimar $\boldsymbol{\beta}$ permite que a estrutura de correlação entre as observações da mesma unidade experimental seja especificada de diferentes formas: identidade, padrão uniforme, auto-regressiva de ordem 1 e não-estruturada, entre outras. Conforme assumimos na equação de estimação (2.4), $\mathbf{R}(\boldsymbol{\alpha})$ reflete as correlações entre $\mathrm{y}_{i j}^{*}$ e $\mathrm{y}_{i l}^{*}$, ou analogamente, podemos dizer que essa matriz de correlação de trabalho reflete as correlações entre $\mathrm{b}_{i j}$ e $\mathrm{b}_{i l}$, com

$$
\mathrm{b}_{i j}=\mathrm{y}_{i j}^{*}-\mu_{i j}^{*}
$$

para $i=1, \ldots, n$ e $j, l=1, \ldots, t, \operatorname{com} j \neq l$.

Dessa forma, seguindo a proposta de Artes (1997, Seção 3.1.4), apresentamos estimadores para algumas estruturas usadas para definir $\mathbf{R}(\boldsymbol{\alpha})$ :

1. A matriz de correlação padrão uniforme assume que $\operatorname{Corr}\left(\mathrm{b}_{i j}, \mathrm{~b}_{i l}\right)=\alpha, \forall j \neq l$ e $1 \leq j, l \leq t$. Então, a estimativa de $\alpha$ no passo $m$ é dada por

$$
\hat{\alpha}^{(m)}=\left\{\frac{\left(\sum_{i=1}^{n} \sum_{j>l}^{t} \hat{\mathrm{b}}_{i j} \hat{\mathrm{b}}_{i l}\right)}{\left(\sum_{i=1}^{n} \sum_{j=1}^{t} \hat{\mathrm{b}}_{i j}^{2}\right)} \frac{n t}{\frac{1}{2} n t(t-1)}\right\}^{(m)}=\left\{\frac{\left(\sum_{i=1}^{n} \sum_{j>l}^{t} \hat{\mathrm{b}}_{i j} \hat{\mathrm{b}}_{i l}\right)}{\left(\sum_{i=1}^{n} \sum_{j=1}^{t} \hat{\mathrm{b}}_{i j}^{2}\right)} \frac{2}{(t-1)}\right\}^{(m)} .
$$

2. A matriz de correlação autoregressiva de primeira ordem, AR-1, especifica que $\operatorname{Corr}\left(\mathrm{b}_{i j}, \mathrm{~b}_{i l}\right)=\alpha^{|j-l|}, 1 \leq j, l \leq t$. Um estimador simples para $\alpha$ é

$$
\hat{\alpha}^{(m)}=\left\{\frac{\left(\sum_{i=1}^{n} \sum_{j=1}^{t-1} \hat{\mathrm{b}}_{i j} \hat{\mathrm{b}}_{i, j+1}\right)}{\left(\sum_{i=1}^{n} \sum_{j=1}^{t-1} \hat{\mathrm{b}}_{i j}^{2} \sum_{i=1}^{n} \sum_{j=2}^{t} \hat{\mathrm{b}}_{i j}^{2}\right)^{1 / 2}}\right\}^{(m)} .
$$

Uma deficiência desse estimador é que utiliza somente a informação sobre a dependência de observações consecutivas de $b_{i j}$. Liang e Zeger (1986) propõem outro 
estimador que leva em conta uma quantidade maior de informação, observando que $\mathrm{E}\left(\hat{\mathrm{b}}_{i j} \hat{\mathrm{b}}_{i l}\right) \cong\left(\operatorname{Var}\left(\hat{\mathrm{b}}_{i j}\right) \operatorname{Var}\left(\hat{\mathrm{b}}_{i l}\right)\right)^{1 / 2} \alpha^{|j-l|}$. Assim, $\alpha$ no passo $m$ pode ser estimado pelo coeficiente angular da regressão em que a variável dependente é $\log \left(\hat{\mathrm{b}}_{i j}^{(m)} \hat{\mathrm{b}}_{i l}^{(m)}\right)$ e a independente é $\log |j-l|$.

3. Quando a matriz de correlação é a não estruturada, $\boldsymbol{\alpha}=\left(\alpha_{12}, \alpha_{13}, \ldots, \alpha_{t-1, t}\right)^{\top}$ é um vetor com $t(t-1) / 2$ componentes, em que $\alpha_{j l}$ corresponde à correlação entre $\mathrm{b}_{i j} \mathrm{e}$ $\mathrm{b}_{i l}, \operatorname{com} i=1, \ldots, n$ e $j, l=1, \ldots, t, \operatorname{com} j<l$. Um estimador para $\alpha_{j l}$ é dado por

$$
\hat{\alpha}_{j l}^{(m)}=\left\{\frac{\left(\sum_{i=1}^{n} \hat{\mathrm{b}}_{i j} \hat{\mathrm{b}}_{i l}\right)}{\left(\sum_{i=1}^{n} \hat{\mathrm{b}}_{i j}^{2}\right)^{1 / 2}\left(\sum_{i=1}^{n} \hat{\mathrm{b}}_{i l}^{2}\right)^{1 / 2}}\right\}^{(m)} .
$$

Com relação à escolha da estrutura da matriz de correlação de trabalho, Liang e Zeger (1986) citam que para qualquer $\mathbf{R}(\boldsymbol{\alpha})$ utilizada, $\hat{\boldsymbol{\beta}}$ e $\hat{\mathbf{J}}_{n}^{-1}$ serão consistentes e a eficiência cresce quanto mais próxima da verdadeira matriz de correlação estiver a escolha da matriz de correlação de trabalho. Wang e Carey (2003) estudam a eficiência relativa assintótica de $\hat{\boldsymbol{\beta}}$ segundo a espeficação: da estrutura da matriz de correlação de trabalho, do estimador de $\boldsymbol{\alpha}$ e da matriz de planejamento (ou de covariáveis). Estudos analíticos e numéricos realizados por esses autores mostram que o principal impacto causado na eficiência dos estimadores de regressão é devido à especificação da estrutura imposta à matriz de correlação de trabalho.

A seguir, apresentamos as etapas que mostram como na prática podemos estimar os parâmetros de $\boldsymbol{\beta}, \phi$ e $\boldsymbol{\alpha}$.

\section{ETAPAS PARA ESTIMAÇÃO DE $\boldsymbol{\beta}, \phi$ E $\boldsymbol{\alpha}$.}

1. Supondo independência entre as observações da mesma unidade experimental, ajustamos um modelo de regressão linear de $g(\mathbf{y})$ sobre $\mathbf{X}$ via método de mínimos quadrados ordinários, com $\boldsymbol{\mu}=\left(\boldsymbol{\mu}_{1}^{\top}, \ldots, \boldsymbol{\mu}_{n}^{\top}\right)^{\top}$ e $\mathbf{X}=\left(\mathbf{X}_{1}^{\top}, \ldots, \mathbf{X}_{n}^{\top}\right)^{\top}$. Notemos que a suposição de independência elimina os parâmetros $\boldsymbol{\alpha}$ e $\phi$ do processo de estimação de $\boldsymbol{\beta}$.

2. Ainda supondo independência entre as observações da mesma unidade experimental, ou seja, definindo $R(\boldsymbol{\alpha})$ igual a uma matriz identidade, consideramos as estimativas dos parâmetros de regressão da etapa anterior como valores iniciais, $\hat{\boldsymbol{\beta}}^{(0)}$, para 
estimar $\boldsymbol{\beta}$ e $\phi$ por meio das equações (2.6) e (2.7), respectivamente. Essas duas estimativas são calculadas a cada passo do processo iterativo até a convergência de $\boldsymbol{\beta}$. Nesta etapa, obtemos as estimativas de $\boldsymbol{\beta}$ e $\phi$ considerando um modelo de regressão beta com independência entre as observações da mesma unidade experimental.

3. Definimos uma estrutura de correlação de trabalho $\mathbf{R}(\boldsymbol{\alpha})$ para ser utilizada na modelagem dos dados. Se a estrutura for a independente, as estimativas de $\boldsymbol{\beta}$ e $\phi$ são os valores obtidos na convergência do processo iterativo da etapa 2. Caso contrário, considerando como $\hat{\boldsymbol{\beta}}^{(0)}$ e $\hat{\phi}^{(0)}$ as estimativas de $\boldsymbol{\beta}$ e $\phi$ encontradas na etapa 2 , calculamos os parâmetros de correlação $\left(\hat{\boldsymbol{\alpha}}^{(0)}\right)$. No passo seguinte do processo iterativo, calculamos os parâmetros de regressão $\left(\hat{\boldsymbol{\beta}}^{(m)}\right)$ utilizando novamente a equação (2.6), o parâmetro de precisão $\left(\hat{\phi}^{(m)}\right)$ e os parâmetros de correlação $\left(\hat{\boldsymbol{\alpha}}^{(m)}\right)$. Repetimos esse passo do processo até a convergência de $\boldsymbol{\beta}$.

\subsection{Modelagem conjunta dos parâmetros de posição e precisão}

Seja $\mathbf{y}_{i}=\left(\mathrm{y}_{i 1}, \mathrm{y}_{i 2}, \ldots, \mathrm{y}_{i t_{i}}\right)^{\top}$ o vetor $\left(t_{i} \times 1\right)$ de respostas da $i$-ésima unidade experimental, com $i=1, \ldots, n$. Vamos assumir que a densidade marginal da variável resposta tenha distribuição beta, $\mathrm{y}_{i j} \sim \mathcal{B}\left(\mu_{i j}, \phi_{i j}\right)$, dada por

$$
p\left(\mathrm{y}_{i j} ; \mu_{i j}, \phi_{i j}\right)=\frac{\Gamma\left(\phi_{i j}\right)}{\Gamma\left(\mu_{i j} \phi_{i j}\right) \Gamma\left(\left(1-\mu_{i j}\right) \phi_{i j}\right)} \mathrm{y}_{i j}^{\mu_{i j} \phi_{i j}-1}\left(1-\mathrm{y}_{i j}\right)^{\left(1-\mu_{i j}\right) \phi_{i j}-1}
$$

Aqui, incorporamos a heterogeneidade do parâmetro de precisão, ou seja, cada observação $\mathrm{y}_{i j}$ assume ter sua própria precisão, $\phi_{i j}, i=1, \ldots, n$ e $j=1, \ldots, t_{i}$.

Consideremos que o componente sistemático que modela a média, $\mu_{i j}$, seja dado pela equação (2.2) e que o componente sistemático que modela o parâmetro de precisão, $\phi_{i j}$, como função de covariáveis $\mathbf{q}_{i j}$ (que podem ser um subconjunto de $\mathbf{x}_{i j}$ ), seja dado por

$$
f\left(\phi_{i j}\right)=\delta_{i j}=\mathbf{q}_{i j}^{\top} \gamma,
$$

sendo $f($.$) uma função monótona e duplamente diferenciável cuja inversa deve ser positiva,$ $\delta_{i j}$ o preditor linear e $\gamma=\left(\gamma_{1}, \ldots, \gamma_{q}\right)^{\top}, q<n$, um vetor de parâmetros desconhecidos a serem estimados, com $i=1, \ldots, n$ e $j=1, \ldots, t_{i}$. Para facilitar a notação, assumimos, sem perda de generalidade, que $t_{i}=t, i=1, \ldots, n$. 
A construção de equações de estimação para modelagem conjunta dos parâmetros de posição e precisão, no contexto de modelos de regressão beta com medidas repetidas, considera a densidade marginal de $\mathrm{y}_{i j}$ descrita na equação (2.9), os componentes sistemáticos (2.2) e (2.10) que modelam, respectivamente, os parâmetros de posição e de precisão e os vetores $\mathbf{b}_{i}=\mathbf{y}_{i}^{*}-\boldsymbol{\mu}_{i}^{*}, i=1, \ldots, n$. Notemos que esses vetores são funções tanto do vetor de parâmetros $\boldsymbol{\beta}$ como do vetor de parâmetros $\boldsymbol{\gamma}$ e sua utilização é justificada na Seção 2.1 .

Logo, assumindo dependência entre as observações da mesma unidade experimental $i$, a função de estimação ótima de $\boldsymbol{\theta}=\left(\boldsymbol{\beta}^{\top}, \boldsymbol{\gamma}^{\top}\right)^{\top}$, obtida a partir de (1.14), é equivalente a

$$
\Psi_{2}^{o}(\boldsymbol{\theta})=\sum_{i=1}^{n}\left(\begin{array}{c}
\mathbf{X}_{i}^{\top} \mathbf{G}_{i} \boldsymbol{\Phi}_{i} \mathbf{A}_{i} \\
\mathbf{Q}_{i}^{\top} \mathbf{F}_{i} \mathbf{C}_{i}
\end{array}\right)\left(\mathbf{A}_{i}^{1 / 2} \mathbf{R}\left(\mathbf{y}_{i}^{*}\right) \mathbf{A}_{i}^{1 / 2}\right)^{-1}\left(\mathbf{y}_{i}^{*}-\boldsymbol{\mu}_{i}^{*}\right)
$$

sendo $\mathbf{X}_{i}=\left(\mathbf{x}_{i 1}, \ldots, \mathbf{x}_{i t}\right)^{\top}, \quad \mathbf{\Phi}_{i}=\operatorname{diag}\left(\phi_{i 1}, \ldots, \phi_{i t}\right), \quad \mathbf{A}_{i}=\operatorname{diag}\left(\mathrm{a}_{i 1}, \ldots, \mathrm{a}_{i t}\right), \quad \mathbf{G}_{i}=$ $\operatorname{diag}\left(\partial g^{-1}\left(\eta_{i 1}\right) / \partial \eta_{i 1}, \ldots, \partial g^{-1}\left(\eta_{i t}\right) / \partial \eta_{i t}\right), \quad \mathbf{Q}_{i}=\left(\mathbf{q}_{i 1}, \ldots, \mathbf{q}_{i t}\right)^{\top}, \quad \mathbf{C}_{i}=\operatorname{diag}\left(c_{i 1}, \ldots, \mathrm{c}_{i t}\right)$, $\mathbf{F}_{i}=\operatorname{diag}\left(\partial f^{-1}\left(\delta_{i 1}\right) / \partial \delta_{i 1}, \ldots, \partial f^{-1}\left(\delta_{i t}\right) / \partial \delta_{i t}\right)$ e $\mathbf{R}\left(\mathbf{y}_{i}^{*}\right)$ a verdadeira matriz de correlação de $\mathbf{y}_{i}^{*}$, em que $\mathrm{a}_{i j}=\psi^{\prime}\left(\mu_{i j} \phi_{i j}\right)+\psi^{\prime}\left(\left(1-\mu_{i j}\right) \phi_{i j}\right)$ e $\mathrm{c}_{i j}=\mu_{i j} \psi^{\prime}\left(\mu_{i j} \phi_{i j}\right)-\left(1-\mu_{i j}\right) \psi^{\prime}((1-$ $\left.\left.\mu_{i j}\right) \phi_{i j}\right), \operatorname{com} i=1, \ldots, n$ e $j=1, \ldots, t$. As contas detalhadas estão apresentadas na Seção A.1.2 do Apêndice A e as possíveis expressões para $\mathbf{G}$ e $\mathbf{F}$ de acordo, respectivamente, com as funções $g(\cdot)$ e $f(\cdot)$ adotadas estão apresentadas na Tabela C.1 do Apêndice C.

Sob as condições do Teorema 1 , temos que $\hat{\boldsymbol{\theta}}$, solução de $\boldsymbol{\Psi}_{2}^{o}(\hat{\boldsymbol{\theta}})=\mathbf{0}$, é um estimador consistente de $\boldsymbol{\theta}$ e que

$$
\sqrt{n}(\hat{\boldsymbol{\theta}}-\boldsymbol{\theta}) \stackrel{\mathcal{D}}{\longrightarrow} \mathrm{N}_{p+q}\left(\mathbf{0}, \lim _{n \rightarrow \infty} n\left\{\sum_{i=1}^{n}\left(\begin{array}{c}
\mathbf{X}_{i}^{\top} \mathbf{G}_{i} \boldsymbol{\Phi}_{i} \mathbf{A}_{i} \\
\mathbf{Q}_{i}^{\top} \mathbf{F}_{i} \mathbf{C}_{i}
\end{array}\right) \operatorname{Cov}\left(\mathbf{b}_{i}\right)^{-1}\left(\begin{array}{c}
\mathbf{X}_{i}^{\top} \mathbf{G}_{i} \boldsymbol{\Phi}_{i} \mathbf{A}_{i} \\
\mathbf{Q}_{i}^{\top} \mathbf{F}_{i} \mathbf{C}_{i}
\end{array}\right)^{\top}\right\}^{-1}\right) .
$$

Conforme ressaltamos na Seção 2.1, iremos substituir a verdadeira matriz de correlação por uma matriz de correlação de trabalho, $\mathbf{R}(\boldsymbol{\alpha})$, que satisfaça as condições para ser uma matriz de correlação, em que $\boldsymbol{\alpha}$, um vetor $(s \times 1)$, caracteriza completamente $\mathbf{R}(\boldsymbol{\alpha})$. Notemos que $\mathbf{R}(\boldsymbol{\alpha})$ não precisa ser necessariamente a verdadeira matriz de correlação dos $\mathbf{y}_{i}^{*}$ 's.

Com isso, a função de estimação generalizada de $\boldsymbol{\theta}$ é dada por

$$
\begin{aligned}
\boldsymbol{\Psi}_{2}(\boldsymbol{\theta}) & =\left(\begin{array}{c}
\boldsymbol{\Psi}_{2}(\boldsymbol{\beta}) \\
\boldsymbol{\Psi}_{2}(\boldsymbol{\gamma})
\end{array}\right)=\sum_{i=1}^{n}\left(\begin{array}{c}
\mathbf{X}_{i}^{\top} \mathbf{G}_{i} \boldsymbol{\Phi}_{i} \mathbf{A}_{i} \\
\mathbf{Q}_{i}^{\top} \mathbf{F}_{i} \mathbf{C}_{i}
\end{array}\right) \boldsymbol{\Omega}_{i}^{-1}\left(\mathbf{y}_{i}^{*}-\boldsymbol{\mu}_{i}^{*}\right)= \\
& =\sum_{i=1}^{n} \mathbf{M}_{i}^{\top} \boldsymbol{\Lambda}_{i} \boldsymbol{\Omega}_{i}^{-1} \mathbf{b}_{i}=\sum_{i=1}^{n} \mathbf{M}_{i}^{\top} \mathbf{W}_{i} \boldsymbol{\Lambda}_{i}^{-\top} \mathbf{b}_{i},
\end{aligned}
$$


sendo $\boldsymbol{\Lambda}_{i}^{-\top}$ a inversa generalizada de $\boldsymbol{\Lambda}_{i}^{\top}, \mathbf{M}_{i}=\left(\begin{array}{cc}\mathbf{X}_{i} & \mathbf{0} \\ \mathbf{0} & \mathbf{Q}_{i}\end{array}\right), \quad \boldsymbol{\Lambda}_{i}=\left(\begin{array}{c}\mathbf{G}_{i} \mathbf{\Phi}_{i} \mathbf{A}_{i} \\ \mathbf{F}_{i} \mathbf{C}_{i}\end{array}\right)$, $\boldsymbol{\Omega}_{i}=\mathbf{A}_{i}^{1 / 2} \mathbf{R}(\boldsymbol{\alpha}) \mathbf{A}_{i}^{1 / 2}$ e $\mathbf{W}_{i}=\boldsymbol{\Lambda}_{i} \boldsymbol{\Omega}_{i}^{-1} \boldsymbol{\Lambda}_{i}^{\top}$. Como $\boldsymbol{\Lambda}_{i}^{\top}$ é uma matriz com dimensão $t \times 2 t$ com posto $t$, então temos que $\boldsymbol{\Lambda}_{i}^{\top} \boldsymbol{\Lambda}_{i}^{-\top}=\mathbf{I}_{t}$, sendo $\mathbf{I}_{t}$ uma matriz identidade de tamanho $t$ (Graybill, 1976, Teorema 1.5.12). Notemos que apenas nesta seção, $\boldsymbol{\Lambda}_{i}$ não é definida como uma matriz simétrica.

Como a equação de estimação dada em (2.13) deixa de ser a ótima, no próximo teorema descrevemos algumas condições que o estimador de $\boldsymbol{\alpha}$ deve satisfazer de modo que o estimador de $\boldsymbol{\theta}$ seja consistente e assintoticamente normal.

Teorema 3. Seja $\hat{\boldsymbol{\theta}}$ a raiz de (2.13). Sob condições gerais de regularidade e assumindo que $\hat{\boldsymbol{\alpha}}(\boldsymbol{\theta})$ é um estimador $\sqrt{n}$-consistente de $\boldsymbol{\alpha}$ dado $\boldsymbol{\theta}$, então temos que $\hat{\boldsymbol{\theta}}$ é um estimador consistente de $\boldsymbol{\theta} e$

$$
\sqrt{n}(\hat{\boldsymbol{\theta}}-\boldsymbol{\theta}) \stackrel{\mathcal{D}}{\longrightarrow} \mathrm{N}_{p+q}\left(\mathbf{0}, \boldsymbol{J}^{-1}\right)
$$

com $\boldsymbol{J}=\lim _{n \rightarrow \infty} \boldsymbol{J}_{n} / n$, sendo $\boldsymbol{J}_{n}$ a matriz de informação de Godambe de $\boldsymbol{\theta}$ associada a $\mathbf{\Psi}_{2}$, dada por

$$
\boldsymbol{J}_{n}=\left\{\sum_{i=1}^{n} \boldsymbol{S}_{i}\right\}\left\{\sum_{i=1}^{n} \boldsymbol{V}_{i}\right\}^{-1}\left\{\sum_{i=1}^{n} \boldsymbol{S}_{i}\right\}
$$

em que

$$
\sum_{i=1}^{n} \boldsymbol{S}_{i}=E\left[\frac{\partial}{\partial \boldsymbol{\theta}^{\top}} \boldsymbol{\Psi}_{2}(\boldsymbol{\theta})\right]=-\sum_{i=1}^{n} \boldsymbol{M}_{i}^{\top} \boldsymbol{W}_{i} \boldsymbol{M}_{i}
$$

$e$

$$
\sum_{i=1}^{n} \boldsymbol{V}_{i}=E\left[\boldsymbol{\Psi}_{2}(\boldsymbol{\theta}) \boldsymbol{\Psi}_{2}^{\top}(\boldsymbol{\theta})\right]=\sum_{i=1}^{n} \boldsymbol{M}_{i}^{\top} \boldsymbol{\Lambda}_{i} \boldsymbol{\Omega}_{i}^{-1} \operatorname{Cov}\left(\boldsymbol{b}_{i}\right) \boldsymbol{\Omega}_{i}^{-1} \boldsymbol{\Lambda}_{i}^{\top} \boldsymbol{M}_{i}
$$

A prova desse teorema é análoga à encontrada em Artes (1997, Teorema 7, p.67).

No caso de ajustar conjuntamente os parâmetros de posição e precisão segundo a equação (2.13), o estimador robusto para a matriz de covariâncias de $\hat{\boldsymbol{\theta}}$ é dado por

$$
\hat{\mathbf{J}}_{n}^{-1}=\left\{\sum_{i=1}^{n} \hat{\mathbf{S}}_{i}\right\}^{-1}\left\{\sum_{i=1}^{n} \mathbf{M}_{i}^{\top} \hat{\mathbf{\Lambda}}_{i} \hat{\mathbf{\Omega}}_{i}^{-1} \hat{\mathbf{b}}_{i} \hat{\mathbf{b}}_{i}^{\top} \hat{\mathbf{\Omega}}_{i}^{-1} \hat{\mathbf{\Lambda}}_{i}^{\top} \mathbf{M}_{i}\right\}\left\{\sum_{i=1}^{n} \hat{\mathbf{S}}_{i}\right\}^{-1} .
$$

A estimativa da expressão acima é obtida substituindo $\boldsymbol{\theta}$ e $\boldsymbol{\alpha}$ por suas respectivas estimativas consistentes. Já o estimador "naive", obtido quando $\mathbf{R}(\boldsymbol{\alpha})$ representar a verdadeira 
matriz de correlação dos $\mathbf{y}_{i}^{*}$ s, é dado por

$$
\hat{\mathbf{J}}_{n}^{-1}=-\left\{\sum_{i=1}^{n} \hat{\mathbf{S}}_{i}\right\}^{-1} .
$$

\subsubsection{Estimação de $\beta, \gamma$ e $\alpha$}

O processo iterativo para calcular $\hat{\boldsymbol{\theta}}=\left(\hat{\boldsymbol{\beta}}^{\top}, \hat{\boldsymbol{\gamma}}^{\top}\right)^{\top}$ e $\hat{\boldsymbol{\alpha}}$ combina o método scoring de Fisher para estimar os parâmetros de regressão $\boldsymbol{\theta}=\left(\boldsymbol{\beta}^{\top}, \boldsymbol{\gamma}^{\top}\right)^{\top}$ com o método dos momentos para estimar os parâmetros de correlação $\boldsymbol{\alpha}$. Expandindo a EEG dada na equação (2.13) em torno de um valor inicial $\hat{\boldsymbol{\theta}}^{(0)}=\left(\hat{\boldsymbol{\beta}}^{(0) \top}, \hat{\boldsymbol{\gamma}}^{(0) \top}\right)^{\top}$, o processo iterativo para estimar $\boldsymbol{\theta}$ é dado por

$$
\begin{aligned}
\hat{\boldsymbol{\theta}}^{(m+1)} & =\hat{\boldsymbol{\theta}}^{(m)}-\left\{\mathrm{E}\left[\frac{\partial}{\partial \boldsymbol{\theta}^{\top}} \mathbf{\Psi}_{2}\left(\hat{\boldsymbol{\theta}}^{(m)}\right)\right]\right\}^{-1} \mathbf{\Psi}_{2}\left(\hat{\boldsymbol{\theta}}^{(m)}\right)= \\
& =\hat{\boldsymbol{\theta}}^{(m)}+\left\{\left[\sum_{i=1}^{n} \mathbf{M}_{i}^{\top} \hat{\mathbf{W}}_{i} \mathbf{M}_{i}\right]^{-1}\left[\sum_{i=1}^{n} \mathbf{M}_{i}^{\top} \hat{\mathbf{W}}_{i} \hat{\boldsymbol{\Lambda}}_{i}^{-\top} \hat{\mathbf{b}}_{i}\right]\right\}^{(m)}
\end{aligned}
$$

sendo $m=0,1,2, \ldots$ o número de iterações. O índice $m$ no lado direito das equações acima indica que as matrizes e os vetores são atualizados pelas estimativas de $\boldsymbol{\theta}$ e $\boldsymbol{\alpha}$ da $m$-ésima iteração.

Reescrevendo (2.14) na forma de um processo iterativo de mínimos quadrados reponderados que emprega uma matriz de pesos $\mathbf{W}_{i}$ e uma variável dependente modificada $\mathbf{z}_{i}$, temos:

$$
\hat{\boldsymbol{\theta}}^{(m+1)}=\left\{\left[\sum_{i=1}^{n} \mathbf{M}_{i}^{\top} \hat{\mathbf{W}}_{i} \mathbf{M}_{i}\right]^{-1}\left[\sum_{i=1}^{n} \mathbf{M}_{i}^{\top} \hat{\mathbf{W}}_{i} \mathbf{z}_{i}\right]\right\}^{(m)}
$$

sendo $\mathbf{z}_{i}=\hat{\boldsymbol{\nu}}_{i}+\hat{\boldsymbol{\Lambda}}_{i}^{-\top} \hat{\mathbf{b}}_{i}, \operatorname{com} \boldsymbol{\nu}_{i}=\left(\boldsymbol{\eta}_{i}^{\top}, \boldsymbol{\delta}_{i}^{\top}\right)^{\top}$.

Para estimar $\boldsymbol{\alpha}$, que reflete as correlações entre $\mathrm{y}_{i j}^{*}$ e $\mathrm{y}_{i l}^{*}$, para $i=1, \ldots, n$ e $j, l=$ $1, \ldots, t, \operatorname{com} j \neq l$, procedemos conforme descrito na Seção 2.1.1.

A seguir, apresentamos as etapas que mostram como na prática podemos estimar os parâmetros de $\boldsymbol{\beta}, \boldsymbol{\gamma}$ e $\boldsymbol{\alpha}$.

Etapas PARA ESTIMAÇÃO DE $\boldsymbol{\beta}, \boldsymbol{\gamma}$ E $\boldsymbol{\alpha}$.

1. Supondo independência entre as observações da mesma unidade experimental, ajustamos um modelo de regressão linear de $g(\mathbf{y})$ sobre $\mathbf{X}$ e um de $f(\check{\phi})$ sobre $\mathbf{Q}$, 
ambos via método de mínimos quadrados ordinários, sendo $\boldsymbol{\phi}=\left(\boldsymbol{\phi}_{1}^{\top}, \ldots, \boldsymbol{\phi}_{n}^{\top}\right)^{\top} \mathrm{e}$ $\mathbf{Q}=\left(\mathbf{Q}_{1}^{\top}, \ldots, \mathbf{Q}_{n}^{\top}\right)^{\top}$, com $\phi_{i}=\left(\phi_{i 1}, \ldots, \phi_{i t}\right)^{\top}$ e $i=1, \ldots, n$. Além disso, para o ajuste do parâmetro de precisão, utilizamos como valor de $\phi_{i j}$, $\check{\phi}_{i j}$, a sugestão proposta por Ferrari e Cribari-Neto (2004) que é dada por:

$$
\check{\phi}_{i j}=\frac{\check{\mu}_{i j}\left(1-\check{\mu}_{i j}\right)}{\check{\sigma}_{i j}^{2}}-1 \text {, }
$$

em que

$$
\check{\mu}_{i j}=g^{-1}\left(\mathbf{x}_{i j}^{\top} \hat{\boldsymbol{\beta}}_{\mathrm{MQO}}\right) \quad \text { e } \quad \check{\sigma}_{i j}^{2}=\check{\mathbf{e}}^{\top} \check{\mathbf{e}}\left[\check{\mathrm{G}}_{i j}^{2} /(n-p)\right]
$$

sendo $\hat{\boldsymbol{\beta}}_{\mathrm{MQO}}$ a estimativa obtida pelo ajuste da média via método de mínimos quadrados ordinários, $\check{\mathbf{e}}=g(\mathbf{y})-\mathbf{X} \hat{\boldsymbol{\beta}}_{\mathrm{MQO}}$ o vetor de resíduos de mínimos quadrados ordinários de uma regressão linear com a variável resposta transformada e $\check{\mathrm{G}}_{i j} \mathrm{o}$ $j$-ésimo elemento da diagonal de $\mathbf{G}_{i}$ avaliado em $\check{\mu}_{i j}$.

2. Uma estrutura de correlação de trabalho $\mathbf{R}(\boldsymbol{\alpha})$ deve ser definida para ser utilizada na modelagem dos dados. Considerando as estimativas dos parâmetros de regressão da etapa anterior como valores iniciais, $\hat{\boldsymbol{\beta}}^{(0)}$ e $\hat{\boldsymbol{\gamma}}^{(0)}$, obtemos a estimativa inicial de $\boldsymbol{\alpha}, \hat{\boldsymbol{\alpha}}^{(0)}$, levando em conta os componentes do vetor $\mathbf{b}_{i}$ expresso em (2.8). Nesta etapa, estimamos $\boldsymbol{\beta}$ e $\boldsymbol{\gamma}$ em processos iterativos separados dados, respectivamente, por

$$
\hat{\boldsymbol{\beta}}^{(m+1)}=\left\{\left[\sum_{i=1}^{n} \mathbf{X}_{i}^{\top} \hat{\mathbf{W}}_{\beta i} \mathbf{X}_{i}\right]^{-1}\left[\sum_{i=1}^{n} \mathbf{X}_{i}^{\top} \hat{\mathbf{W}}_{\beta i} \mathbf{z}_{\beta i}\right]\right\}^{(m)}
$$

$\operatorname{com} \hat{\mathbf{W}}_{\beta i}=\hat{\mathbf{G}}_{i} \hat{\boldsymbol{\Phi}}_{i} \hat{\mathbf{A}}_{i} \hat{\boldsymbol{\Omega}}_{i}^{-1} \hat{\mathbf{A}}_{i} \hat{\boldsymbol{\Phi}}_{i} \hat{\mathbf{G}}_{i}$ e $\mathbf{z}_{\beta i}=\hat{\boldsymbol{\eta}}_{i}+\left(\hat{\mathbf{A}}_{i} \hat{\boldsymbol{\Phi}}_{i} \hat{\mathbf{G}}_{i}\right)^{-1} \hat{\mathbf{b}}_{i}$, e por

$$
\hat{\boldsymbol{\gamma}}^{(m+1)}=\left\{\left[\sum_{i=1}^{n} \mathbf{Q}_{i}^{\top} \hat{\mathbf{W}}_{\gamma i} \mathbf{Q}_{i}\right]^{-1}\left[\sum_{i=1}^{n} \mathbf{Q}_{i}^{\top} \hat{\mathbf{W}}_{\gamma i} \mathbf{z}_{\gamma i}\right]\right\}^{(m)},
$$

$\operatorname{com} \hat{\mathbf{W}}_{\gamma i}=\hat{\mathbf{F}}_{i} \hat{\mathbf{C}}_{i} \hat{\boldsymbol{\Omega}}_{i}^{-1} \hat{\mathbf{C}}_{i} \hat{\mathbf{F}}_{i}$ e $\mathbf{z}_{\gamma i}=\hat{\boldsymbol{\delta}}_{i}+\left(\hat{\mathbf{C}}_{i} \hat{\mathbf{F}}_{i}\right)^{-1} \hat{\mathbf{b}}_{i}$. Esses processos são escritos separando as equações de estimação de $\boldsymbol{\beta}$ e de $\boldsymbol{\gamma}, \boldsymbol{\Psi}_{2}(\boldsymbol{\beta})$ e $\boldsymbol{\Psi}_{2}(\boldsymbol{\gamma})$, definidas em (2.12). As estimativas de $\boldsymbol{\beta}, \boldsymbol{\gamma}$ e $\boldsymbol{\alpha}$ são calculadas a cada passo $m$ até a convergência de $\boldsymbol{\beta}$ e $\boldsymbol{\gamma}$.

3. Consideramos as estimativas de $\boldsymbol{\beta}, \boldsymbol{\gamma}$ e $\boldsymbol{\alpha}$ da etapa anterior como valores iniciais $\hat{\boldsymbol{\beta}}^{(0)}, \hat{\boldsymbol{\gamma}}^{(0)}$ e $\hat{\boldsymbol{\alpha}}^{(0)}$, do processo iterativo dado em (2.14). Repetimos cada passo $(m)$ do processo iterativo até a convergência de $\boldsymbol{\beta}$ e $\boldsymbol{\gamma}$. 


\section{Capítulo 3}

\section{Equações de estimação para modelos de regressão simplex com medidas}

\section{repetidas}

A distribuição simplex pertence à classe de modelos de dispersão (J $\phi$ rgensen, 1997) e, assim como a distribuição beta, tem o suporte definido no intervalo unitário $(0,1)$. Com o intuito de modelar dados com medidas repetidas definidos nesse intervalo, apresentamos equações de estimação generalizadas para modelos de regressão simplex baseadas nas propostas de Artes (1997), Artes e J $\phi$ rgensen (2000), Song e Tan (2000) e Song et al. (2004).

A seguir, modelamos a média considerando a homogeneidade do parâmetro de dispersão. Na Seção 3.2, a média e a dispersão são modeladas conjuntamente via equações de estimação, com o intuito de incorporar uma possível heterogeneidade da dispersão.

\subsection{Modelagem do parâmetro de posição}

Seja $\mathbf{y}_{i}=\left(\mathrm{y}_{i 1}, \mathrm{y}_{i 2}, \ldots, \mathrm{y}_{i t_{i}}\right)^{\top}$ o vetor de respostas da $i$-ésima unidade experimental, com $i=1, \ldots, n$. Vamos assumir que a variável resposta $\mathrm{y}_{i j}$ tenha distribuição simplex com média $\mu_{i j}$ e parâmetro de dispersão $\phi^{-1}, \mathrm{y}_{i j} \sim \mathcal{S}^{-}\left(\mu_{i j}, \phi\right)$, com função densidade de probabilidade conforme descrita em (1.7). A princípio, vamos considerar que o parâmetro de dispersão $\phi^{-1}$, além de ser igual para todas as observações, é conhecido.

Consideremos que as médias $\mu_{i j}$ 's são modeladas pelo componente sistemático dado 
na equação (2.2). Para facilitar a notação, assumimos, sem perda de generalidade, que $t_{i}=t, i=1, \ldots, n$.

Para construir equações de estimação, de acordo com a definição de função de estimação linear ótima dada em (1.14), é necessário ter vetores $\mathbf{b}_{1 i}=\mathbf{b}_{1 i}\left(\mathbf{y}_{i} ; \boldsymbol{\beta}\right), i=1, \ldots, n$, com média zero, mutuamente independentes e com as mesmas propriedades das funções de estimação regulares definidas na Seção 1.3. Além disso, vamos definí-los de tal forma que, no caso de independência entre todas as observações, gerem uma classe $\mathcal{L}(\mathbf{b})$ que contenha a função escore dada em (1.11).

Assim, no contexto de modelos de regressão simplex com medidas repetidas, definimos $\mathbf{b}_{1 i}=\mathbf{u}_{i}=\mathbf{u}_{i}\left(\mathbf{y}_{i} ; \boldsymbol{\mu}_{i}\right), \operatorname{com} \mathbf{u}_{i}=\left(\mathrm{u}_{i 1}, \ldots, \mathrm{u}_{i t}\right)^{\top}, \mathbf{y}_{i}=\left(\mathrm{y}_{i 1}, \ldots, \mathrm{y}_{i t}\right)^{\top}$ e $\boldsymbol{\mu}_{i}=\left(\mu_{i 1}, \ldots, \mu_{i t}\right)^{\top}$, sendo

$$
\mathrm{u}_{i j}=-\frac{1}{2} \frac{\partial d\left(\mathrm{y}_{i j} ; \mu_{i j}\right)}{\partial \mu_{i j}}=\frac{\mathrm{y}_{i j}-\mu_{i j}}{\mu_{i j}\left(1-\mu_{i j}\right)}\left[d\left(\mathrm{y}_{i j} ; \mu_{i j}\right)+\frac{1}{\mu_{i j}^{2}\left(1-\mu_{i j}\right)^{2}}\right],
$$

oriundo da função escore dada em (1.11), com $i=1, \ldots, n$ e $j=1, \ldots, t$. Assumindo dependência entre as observações da mesma unidade experimental $i$, os termos da função de estimação ótima definidos em (1.14) são dados por

e

$$
\mathrm{E}\left(\frac{\partial \mathbf{b}_{i}}{\partial \boldsymbol{\beta}^{\top}}\right)^{\top}=-\mathbf{X}_{i}^{\top} \boldsymbol{\Lambda}_{i}
$$

$$
\operatorname{Cov}\left(\mathbf{b}_{i}\right)=\operatorname{Var}\left(\mathbf{u}_{i}\right)^{1 / 2} \mathbf{R}\left(\mathbf{u}_{i}\right) \operatorname{Var}\left(\mathbf{u}_{i}\right)^{1 / 2}=\mathbf{A}_{i}^{1 / 2} \mathbf{R}\left(\mathbf{u}_{i}\right) \mathbf{A}_{i}^{1 / 2}
$$

sendo $\quad \mathbf{X}_{i}=\left(\mathbf{x}_{i 1}, \ldots, \mathbf{x}_{i t}\right)^{\top}, \quad \boldsymbol{\Lambda}_{i}=\phi \mathbf{G}_{i} \mathbf{A}_{i}, \quad \mathbf{A}_{i}=\operatorname{diag}\left(\mathrm{a}_{i 1}, \ldots, \mathrm{a}_{i t}\right)$ e $\mathbf{R}\left(\mathbf{u}_{i}\right)$ a verdadeira matriz de correlação de $\mathbf{u}_{i}, \operatorname{com} \mathbf{G}_{i}=\operatorname{diag}\left(\partial g^{-1}\left(\eta_{i 1}\right) / \partial \eta_{i 1}, \ldots, \partial g^{-1}\left(\eta_{i t}\right) / \partial \eta_{i t}\right)$ e $\mathrm{a}_{i j}=\phi^{-1}\left[3 \phi^{-1} /\left(\mu_{i j}\left(1-\mu_{i j}\right)\right)+1 /\left(\mu_{i j}^{3}\left(1-\mu_{i j}\right)^{3}\right)\right], i=1, \ldots, n$ e $j=1, \ldots, t$. Notemos que, conforme a Proposição 1.1, $\mathrm{a}_{i j}$ é a variância de $\mathrm{u}_{i j}$. As contas detalhadas estão apresentadas na Seção A.2.1 do Apêndice A e as possíveis expressões para $\mathbf{G}$ de acordo com a função de ligação $g(\cdot)$ adotada estão apresentadas na Tabela C.1 do Apêndice C.

Logo, a função de estimação linear ótima de $\boldsymbol{\beta}$, quando consideramos $\phi$ conhecido e a verdadeira matriz de correlação de $\mathbf{u}_{i}$, é equivalente a

$$
\boldsymbol{\Psi}_{1}^{o}(\boldsymbol{\beta})=\sum_{i=1}^{n} \mathbf{X}_{i}^{\top} \boldsymbol{\Lambda}_{i} \operatorname{Cov}\left(\mathbf{u}_{i}\right)^{-1} \mathbf{u}_{i}
$$

Sob as condições do Teorema 1 , temos que $\hat{\boldsymbol{\beta}}$, solução de $\boldsymbol{\Psi}_{1}^{o}(\hat{\boldsymbol{\beta}})=\mathbf{0}$, é um estimador consistente de $\boldsymbol{\beta}$ e que

$$
\sqrt{n}(\hat{\boldsymbol{\beta}}-\boldsymbol{\beta}) \stackrel{\mathcal{D}}{\longrightarrow} \mathrm{N}_{p}\left(\mathbf{0}, \lim _{n \rightarrow \infty} n\left\{\sum_{i=1}^{n} \mathbf{X}_{i}^{\top} \boldsymbol{\Lambda}_{i} \operatorname{Cov}\left(\mathbf{u}_{i}\right)^{-1} \boldsymbol{\Lambda}_{i} \mathbf{X}_{i}\right\}^{-1}\right)
$$


No caso em que a verdadeira matriz de correlação dos vetores $\mathbf{u}_{i}$ 's é desconhecida, definimos uma matriz de correlação de trabalho $\mathbf{R}(\boldsymbol{\alpha})$ (Liang e Zeger, 1986), obtendo a seguinte função de estimação generalizada de $\boldsymbol{\beta}$ :

$$
\boldsymbol{\Psi}_{1}(\boldsymbol{\beta})=\sum_{i=1}^{n} \mathbf{X}_{i}^{\top} \boldsymbol{\Lambda}_{i} \boldsymbol{\Omega}_{i}^{-1} \mathbf{u}_{i}=\sum_{i=1}^{n} \mathbf{X}_{i}^{\top} \mathbf{W}_{i} \boldsymbol{\Lambda}_{i}^{-1} \mathbf{b}_{1 i}
$$

sendo $\boldsymbol{\Omega}_{i}=\mathbf{A}_{i}^{1 / 2} \mathbf{R}(\boldsymbol{\alpha}) \hat{\mathbf{A}}_{i}^{1 / 2}$ e $\quad \mathbf{W}_{i}=\boldsymbol{\Lambda}_{i} \boldsymbol{\Omega}_{i}^{-1} \boldsymbol{\Lambda}_{i}$.

Entretanto, a função de estimação definida em (3.3) deixa de ser a ótima. Nesse caso, é necessário que um estimador de $\boldsymbol{\alpha}$ seja determinado de modo que o estimador de $\boldsymbol{\beta}$, obtido a partir de (3.3), seja consistente e assintoticamente normal. Além disso, como $\phi$ quase sempre é desconhecido, também é necessário propor um estimador para esse parâmetro.

Artes (1997, Teorema 6, pg.47) descreve algumas condições que os estimadores de $\boldsymbol{\alpha}$ e $\phi$ devem satisfazer de modo que a raiz de (3.3), $\hat{\boldsymbol{\beta}}$, seja um estimador consistente de $\boldsymbol{\beta}$ e $\sqrt{n}(\hat{\boldsymbol{\beta}}-\boldsymbol{\beta}) \stackrel{\mathcal{D}}{\longrightarrow} \mathrm{N}_{p}\left(\mathbf{0}, \mathbf{J}^{-1}\right)$, com $\mathbf{J}=\lim _{n \rightarrow \infty} \mathbf{J}_{n} / n$, sendo $\mathbf{J}_{n}$ a matriz de informação de Godambe de $\boldsymbol{\beta}$ associada a $\boldsymbol{\Psi}_{1}$ dada por

$$
\mathbf{J}_{n}=\left\{\sum_{i=1}^{n} \mathbf{S}_{i}\right\}\left\{\sum_{i=1}^{n} \mathbf{V}_{i}\right\}^{-1}\left\{\sum_{i=1}^{n} \mathbf{S}_{i}\right\}
$$

em que

$$
\sum_{i=1}^{n} \mathbf{S}_{i}=\mathrm{E}\left[\frac{\partial}{\partial \boldsymbol{\beta}^{\top}} \boldsymbol{\Psi}_{1}(\boldsymbol{\beta})\right]=-\sum_{i=1}^{n} \mathbf{X}_{i}^{\top} \mathbf{W}_{i} \mathbf{X}_{i}
$$

e

$$
\sum_{i=1}^{n} \mathbf{V}_{i}=\mathrm{E}\left[\Psi_{1}(\boldsymbol{\beta}) \Psi_{1}^{\top}(\boldsymbol{\beta})\right]=\sum_{i=1}^{n} \mathbf{X}_{i}^{\top} \boldsymbol{\Lambda}_{i} \boldsymbol{\Omega}_{i}^{-1} \operatorname{Cov}\left(\mathbf{u}_{i}\right) \boldsymbol{\Omega}_{i}^{-1} \boldsymbol{\Lambda}_{i} \mathbf{X}_{i}
$$

Assim, o estimador robusto de $\mathbf{J}_{n}^{-1}$ é dado por

$$
\hat{\mathbf{J}}_{n}^{-1}=\left\{\sum_{i=1}^{n} \hat{\mathbf{S}}_{i}\right\}^{-1}\left\{\sum_{i=1}^{n} \hat{\mathbf{X}}_{i}^{\top} \hat{\mathbf{G}}_{i} \hat{\mathbf{\Omega}}_{i}^{-1} \hat{\mathbf{b}}_{1 i} \hat{\mathbf{b}}_{1 i}^{\top} \hat{\mathbf{\Omega}}_{i}^{-1} \hat{\mathbf{G}}_{i} \hat{\mathbf{X}}_{i}\right\}\left\{\sum_{i=1}^{n} \hat{\mathbf{S}}_{i}\right\}^{-1},
$$

e o "naive" por $\hat{\mathbf{J}}_{n}^{-1}=-\left\{\sum_{i=1}^{n} \hat{\mathbf{S}}_{i}\right\}^{-1}$. As estimativas das duas últimas expressões são obtidas substituindo $\boldsymbol{\beta}, \boldsymbol{\alpha}$ e $\phi$ por suas respectivas estimativas consistentes. Vale enfatizar que esses resultados assintóticos são válidos mesmo quando $\mathbf{R}(\boldsymbol{\alpha})$ não corresponde à verdadeira matriz de correlação de $\mathbf{u}_{i}, i=1, \ldots, n$, (Artes, 1997). 


\subsubsection{Estimação de $\beta, \phi$ e $\alpha$}

Expandindo a EEG dada na equação (3.3) em torno de um valor inicial $\hat{\boldsymbol{\beta}}^{(0)}$, o processo iterativo para estimar $\boldsymbol{\beta}$ é dado por

$$
\hat{\boldsymbol{\beta}}^{(m+1)}=\hat{\boldsymbol{\beta}}^{(m)}+\left\{\left[\sum_{i=1}^{n} \mathbf{X}_{i}^{\top} \hat{\mathbf{W}}_{i} \mathbf{X}_{i}\right]^{-1}\left[\sum_{i=1}^{n} \mathbf{X}_{i}^{\top} \hat{\mathbf{W}}_{i} \hat{\boldsymbol{\Lambda}}_{i}^{-1} \hat{\mathbf{b}}_{1 i}\right]\right\}^{(m)}
$$

sendo $m=0,1,2, \ldots$ o número de iterações. O índice $m$ no lado direito das equações acima indica que as matrizes e os vetores são atualizados pelas estimativas de $\boldsymbol{\beta}, \boldsymbol{\alpha}$ e $\phi$ da $m$-ésima iteração. A equação (3.4) reescrita na forma de um processo iterativo de mínimos quadrados reponderados fica expressa por

$$
\hat{\boldsymbol{\beta}}^{(m+1)}=\left\{\left[\sum_{i=1}^{n} \mathbf{X}_{i}^{\top} \hat{\mathbf{W}}_{i} \mathbf{X}_{i}\right]^{-1}\left[\sum_{i=1}^{n} \mathbf{X}_{i}^{\top} \hat{\mathbf{W}}_{i} \mathbf{z}_{i}\right]\right\}^{(m)},
$$

que emprega uma matriz de pesos $\mathbf{W}_{i}$ e uma variável dependente modificada $\mathbf{z}_{i}$, sendo $\mathbf{z}_{i}=\hat{\boldsymbol{\eta}}_{i}+\hat{\boldsymbol{\Lambda}}_{i}^{-1} \hat{\mathbf{b}}_{1 i}$. O processo iterativo escrito conforme a equação (3.5) será útil, principalmente, para obtermos as medidas de diagnóstico descritas no Capítulo 5.

Utilizando o método dos momentos e o resultado da equação (1.10), a estimativa de $\phi^{-1}$ obtida no $m$-ésimo passo do processo iterativo é dada por

$$
\hat{\phi}^{-(m)}=\left\{\sum_{i=1}^{n} \sum_{j=1}^{t} d\left(\mathrm{y}_{i j}, \hat{\mu}_{i j}^{(m)}\right) /(n t-p)\right\} .
$$

Aqui, $\boldsymbol{\alpha}$ reflete as correlações entre $\mathrm{u}_{i j}$ e $\mathrm{u}_{i l}$, para $i=1, \ldots, n$ e $j, l=1, \ldots, t$, com $j \neq l$. Logo, para estimar $\boldsymbol{\alpha}$, procedemos conforme descrito na Seção 2.1.1, substituindo $\mathrm{b}_{i j}$ dado na equação $(2.8)$ por

$$
\mathrm{b}_{1 i j}=\mathrm{u}_{i j}
$$

sendo $\mathrm{u}_{i j}$ conforme descrito na equação $(3.1)$, com $i=1, \ldots, n$ e $j=1, \ldots, t$.

A seguir, apresentamos as etapas que mostram como na prática podemos estimar os parâmetros de $\boldsymbol{\beta}, \phi$ e $\boldsymbol{\alpha}$.

ETAPAS PARA ESTIMAÇÃO DE $\boldsymbol{\beta}, \phi$ E $\boldsymbol{\alpha}$.

1. Supondo independência entre as observações da mesma unidade experimental, ajustamos um modelo de regressão linear de $g(\mathbf{y})$ sobre $\mathbf{X}$ via método de mínimos quadrados ordinários, com $\boldsymbol{\mu}=\left(\boldsymbol{\mu}_{1}^{\top}, \ldots, \boldsymbol{\mu}_{n}^{\top}\right)^{\top}$ e $\mathbf{X}=\left(\mathbf{X}_{1}^{\top}, \ldots, \mathbf{X}_{n}^{\top}\right)^{\top}$. Notemos que a suposição de independência elimina os parâmetros $\boldsymbol{\alpha}$ e $\phi$ do processo de estimação de $\boldsymbol{\beta}$. 
2. Ainda supondo independência entre as observações da mesma unidade experimental, ou seja, definindo $R(\boldsymbol{\alpha})$ igual a uma matriz identidade, consideramos as estimativas dos parâmetros de regressão da etapa anterior como valores iniciais, $\hat{\boldsymbol{\beta}}^{(0)}$, para estimar $\boldsymbol{\beta}$ e $\phi$ via equações (3.5) e (3.6), respectivamente. Essas duas estimativas são calculadas a cada passo do processo iterativo até a convergência de $\boldsymbol{\beta}$. Nesta etapa, obtemos as estimativas de $\boldsymbol{\beta}$ e $\phi^{-1}$ considerando um modelo de regressão simplex com independência entre as observações da mesma unidade experimental.

3. Definimos uma estrutura de correlação de trabalho $\mathbf{R}(\boldsymbol{\alpha})$ para ser utilizada na modelagem dos dados. Se a estrutura for a independente, as estimativas de $\boldsymbol{\beta}$ e $\phi$ são os valores obtidos na convergência do processo iterativo da etapa 2. Caso contrário, considerando como $\hat{\boldsymbol{\beta}}^{(0)}$ e $\hat{\phi}^{(0)}$ as estimativas de $\boldsymbol{\beta}$ e $\phi$ encontradas na etapa 2 , calculamos os parâmetros de correlação $\left(\hat{\boldsymbol{\alpha}}^{(0)}\right)$ utilizando o componente expresso em (3.7). No passo seguinte do processo iterativo, calculamos os parâmetros de regressão $\left(\hat{\boldsymbol{\beta}}^{(m)}\right)$ utilizando novamente a equação (3.5), o parâmetro de dispersão $\left(\hat{\phi}^{-(m)}\right)$ pela equação (3.6) e os parâmetros de correlação $\left(\hat{\boldsymbol{\alpha}}^{(m)}\right)$. Repetimos esse passo do processo até a convergência de $\boldsymbol{\beta}$.

\subsection{Modelagem conjunta dos parâmetros de posição e dispersão}

Seja $\mathbf{y}_{i}=\left(\mathrm{y}_{i 1}, \mathrm{y}_{i 2}, \ldots, \mathrm{y}_{i t_{i}}\right)^{\top}$ o vetor $\left(t_{i} \times 1\right)$ de respostas da $i$-ésima unidade experimental, $\operatorname{com} i=1, \ldots, n$. Vamos assumir que a variável resposta $\mathrm{y}_{i j}$ tenha distribuição simplex com média $\mu_{i j}$ e parâmetro de dispersão $\phi_{i j}^{-1}, \mathrm{y}_{i j} \sim \mathcal{S}^{-}\left(\mu_{i j}, \phi_{i j}\right)$, com função densidade de probabilidade conforme descrita em (1.7). Aqui, incorporamos a heterogeneidade do parâmetro de dispersão, ou seja, cada observação $\mathrm{y}_{i j}$ assume ter sua própria dispersão, $\phi_{i j}^{-1}, i=1, \ldots, n$ e $j=1, \ldots, t_{i}$.

Consideremos que o componente sistemático que modela a média, $\mu_{i j}$, seja dado pela equação (2.2) e que o componente sistemático que modela o parâmetro de dispersão, $\phi_{i j}^{-1}$, como função de covariáveis $\mathbf{q}_{i j}$ (que podem ser um subconjunto de $\mathbf{x}_{i j}$ ), seja dado por

$$
f\left(\phi_{i j}^{-1}\right)=\delta_{i j}=\mathbf{q}_{i j}^{\top} \gamma
$$

sendo $f($.$) uma função monótona e duplamente diferenciável com inversa positiva, \delta_{i j}$ o preditor linear e $\gamma=\left(\gamma_{1}, \ldots, \gamma_{q}\right)^{\top}, q<n$, um vetor de parâmetros desconhecidos a serem 
estimados, com $i=1, \ldots, n$ e $j=1, \ldots, t_{i}$. Para facilitar a notação, assumimos, sem perda de generalidade, que $t_{i}=t, i=1, \ldots, n$.

Utilizando a definição de função de estimação linear ótima dada em (1.14) no contexto de modelos de regressão simplex com medidas repetidas, as equações de estimação para estimar a média são construídas baseadas nos vetores $\mathbf{b}_{1 i}=\mathbf{u}_{i}=\mathbf{u}_{i}\left(\mathbf{y}_{i} ; \boldsymbol{\mu}_{i}\right)$ (veja justificativa na Seção 3.1) e para estimar a dispersão, são construídas baseadas nos vetores dados por

$$
\mathbf{b}_{2 i}=\mathbf{d}_{i}-\phi_{i}^{-1}
$$

sendo $\mathbf{d}_{i}=\left(d\left(\mathrm{y}_{i 1} ; \mu_{i 1}\right), \ldots, d\left(\mathrm{y}_{i t} ; \mu_{i t}\right)\right)^{\top}$ e $\phi_{i}^{-1}=\left(\phi_{i 1}^{-1}, \ldots, \phi_{i t}^{-1}\right)^{\top}$, com $i=1, \ldots, n$ e $j=1, \ldots, t$. Os vetores descritos em $(3.9), i=1, \ldots, n$, são vetores com média zero (vide equação (1.10)), mutuamente independentes e com as mesmas propriedades das funções de estimação regulares definidas na Seção 1.3.

Logo, definindo $\mathbf{b}_{i}=\left(\mathbf{b}_{1 i}^{\top}, \mathbf{b}_{2 i}^{\top}\right)^{\top}$ e assumindo dependência entre as observações da mesma unidade experimental $i$, a função de estimação linear ótima de $\boldsymbol{\theta}=\left(\boldsymbol{\beta}^{\top}, \boldsymbol{\gamma}^{\top}\right)^{\top}$ definida a partir de (1.14) é dada por

$$
\boldsymbol{\Psi}_{2}^{o}(\boldsymbol{\theta})=\sum_{i=1}^{n}\left(\begin{array}{cc}
\mathbf{X}_{i}^{\top} \mathbf{G}_{i} \boldsymbol{\Phi}_{i} \mathbf{A}_{i} & \mathbf{0} \\
\mathbf{0} & \mathbf{Q}_{i}^{\top} \mathbf{F}_{i}
\end{array}\right)\left(\begin{array}{cc}
\operatorname{Cov}\left(\mathbf{u}_{i}\right) & \operatorname{Cov}\left(\mathbf{u}_{i}, \mathbf{d}_{i}\right) \\
\operatorname{Cov}\left(\mathbf{d}_{i}, \mathbf{u}_{i}\right) & \operatorname{Cov}\left(\mathbf{d}_{i}\right)
\end{array}\right)^{-1} \mathbf{b}_{i}
$$

em que $\quad \mathbf{X}_{i}=\left(\mathbf{x}_{i 1}, \ldots, \mathbf{x}_{i t}\right)^{\top}, \quad \mathbf{G}_{i}=\operatorname{diag}\left(\partial g^{-1}\left(\eta_{i 1}\right) / \partial \eta_{i 1}, \ldots, \partial g^{-1}\left(\eta_{i t}\right) / \partial \eta_{i t}\right), \quad \mathbf{\Phi}_{i}=$ $\operatorname{diag}\left(\phi_{i 1}, \ldots, \phi_{i t}\right), \quad \mathbf{A}_{i}=\operatorname{diag}\left(\mathrm{a}_{i 1}, \ldots, \mathrm{a}_{i t}\right), \quad \mathbf{F}_{i}=\operatorname{diag}\left(\partial f^{-1}\left(\delta_{i 1}\right) / \partial \delta_{i 1}, \ldots, \partial f^{-1}\left(\delta_{i t}\right) / \partial \delta_{i t}\right)$, $\mathbf{Q}_{i}=\left(\mathbf{q}_{i 1}, \ldots, \mathbf{q}_{i t}\right)^{\top}, \quad \operatorname{Cov}\left(\mathbf{u}_{i}\right)=\mathbf{A}_{i}^{1 / 2} \mathbf{R}\left(\mathbf{u}_{i}\right) \mathbf{A}_{i}^{1 / 2}, \quad \operatorname{Cov}\left(\mathbf{d}_{i}\right)=\mathbf{D}_{i}^{1 / 2} \mathbf{R}\left(\mathbf{d}_{i}\right) \mathbf{D}_{i}^{1 / 2} \quad \mathrm{e}$ $\operatorname{Cov}\left(\mathbf{u}_{i}, \mathbf{d}_{i}\right)$ é a verdadeira matriz de covariâncias entre $\mathbf{u}_{i}$ e $\mathbf{d}_{i}$. Além disso, $\mathbf{R}\left(\mathbf{u}_{i}\right)$ e $\mathbf{R}\left(\mathbf{d}_{i}\right)$ são, respectivamente, as verdadeiras matrizes de correlação de $\mathbf{u}_{i}$ e $\mathbf{d}_{i}, \mathrm{a}_{i j}=$ $\phi_{i j}^{-1}\left[3 \phi_{i j}^{-1} /\left(\mu_{i j}\left(1-\mu_{i j}\right)\right)+1 /\left(\mu_{i j}^{3}\left(1-\mu_{i j}\right)^{3}\right)\right]$ e $\mathbf{D}_{i}=\operatorname{diag}\left(2\left(\phi_{i 1}^{-1}\right)^{2}, \ldots, 2\left(\phi_{i t}^{-1}\right)^{2}\right)$ conforme descrito na Proposição 1.1, com $i=1, \ldots, n$ e $j=1, \ldots, t$. As contas detalhadas estão apresentadas na Seção A.2.2 do Apêndice A e as possíveis expressões para $\mathbf{G}$ e $\mathbf{F}$ de acordo, respectivamente, com as funções $g(\cdot)$ e $f(\cdot)$ adotadas estão apresentadas na Tabela C.1 do Apêndice C.

Por modelar conjuntamente os parâmetros de posição e dispersão, na prática, as equações descritas em (3.10) têm novas quantidades desconhecidas: $\mathbf{R}\left(\mathbf{d}_{i}\right)$ e $\operatorname{Cov}\left(\mathbf{u}_{i}, \mathbf{d}_{i}\right)$. Para solucionar esse problema, são necessárias novas suposições sobre essas estruturas, como por exemplo, substituí-las por matrizes de trabalho como foi feito para a estimação de $\mathbf{R}\left(\mathbf{u}_{i}\right)$ na Seção 3.1. Entretanto, o número de parâmetros de pertubação (nuisance) a serem estimados pode ser reduzido se assumirmos que as equações de estimação são independentes, $\operatorname{Cov}\left(\mathbf{u}_{i}, \mathbf{d}_{i}\right)=\mathbf{0}$; e que não há dependência entre os elementos de $\mathbf{d}_{i}$, 
$\mathbf{R}\left(\mathbf{d}_{i}\right)=\mathbf{I}_{t}$. Isso faz com que afastemo-nos da função de estimação linear ótima definida em (3.10), mas limitamos a quantidade de suposições a serem feitas sobre a estrutura de dependência dos dados (ver detalhes em Artes (1997) e Song et al. (2004)).

Com isso, a função de estimação generalizada de $\boldsymbol{\theta}$ é dada por

$$
\begin{aligned}
\boldsymbol{\Psi}_{2}(\boldsymbol{\theta}) & =\left(\begin{array}{c}
\boldsymbol{\Psi}_{2}(\boldsymbol{\beta}) \\
\boldsymbol{\Psi}_{2}(\boldsymbol{\gamma})
\end{array}\right)=\sum_{i=1}^{n}\left(\begin{array}{c}
\mathbf{X}_{i}^{\top} \mathbf{G}_{i} \boldsymbol{\Phi}_{i} \mathbf{A}_{i}\left(\mathbf{A}_{i}^{1 / 2} \mathbf{R}(\boldsymbol{\alpha}) \mathbf{A}_{i}^{1 / 2}\right)^{-1} \mathbf{b}_{1 i} \\
\mathbf{Q}_{i}^{\top} \mathbf{F}_{i} \mathbf{D}_{i}^{-1} \mathbf{b}_{2 i}
\end{array}\right)= \\
& =\sum_{i=1}^{n} \mathbf{M}_{i}^{\top} \boldsymbol{\Lambda}_{i} \boldsymbol{\Omega}_{i}^{-1} \mathbf{b}_{i}=\sum_{i=1}^{n} \mathbf{M}_{i}^{\top} \mathbf{W}_{i} \boldsymbol{\Lambda}_{i}^{-1} \mathbf{b}_{i}
\end{aligned}
$$

sendo $\mathbf{M}_{i}=\left(\begin{array}{cc}\mathbf{X}_{i} & \mathbf{0} \\ \mathbf{0} & \mathbf{Q}_{i}\end{array}\right), \boldsymbol{\Lambda}_{i}=\left(\begin{array}{cc}\mathbf{G}_{i} \boldsymbol{\Phi}_{i} \mathbf{A}_{i} & \mathbf{0} \\ \mathbf{0} & \mathbf{F}_{i}\end{array}\right), \boldsymbol{\Omega}_{i}=\left(\begin{array}{cc}\mathbf{A}_{i}^{1 / 2} \mathbf{R}(\boldsymbol{\alpha}) \mathbf{A}_{i}^{1 / 2} & \mathbf{0} \\ \mathbf{0} & \mathbf{D}_{i}\end{array}\right) \mathrm{e}$ $\mathbf{W}_{i}=\Lambda_{i} \boldsymbol{\Omega}_{i}^{-1} \Lambda_{i}^{\top}$.

Como a equação de estimação dada em (3.12) deixa de ser a ótima, Artes (1997, Teorema 7, pg.67) descreve algumas condições que o estimador de $\boldsymbol{\alpha}$ deve satisfazer de modo que a raiz de $(3.12), \hat{\boldsymbol{\theta}}$, seja um estimador consistente de $\boldsymbol{\theta}$ e que $\sqrt{n}(\hat{\boldsymbol{\theta}}-$ $\boldsymbol{\theta}) \stackrel{\mathcal{D}}{\longrightarrow} \mathrm{N}_{p+q}\left(\mathbf{0}, \mathbf{J}^{-1}\right)$, com $\mathbf{J}=\lim _{n \rightarrow \infty} \mathbf{J}_{n} / n$, sendo $\mathbf{J}_{n}$ a matriz de informação de Godambe de $\boldsymbol{\theta}$ associada à equação de estimação (3.12).

No caso de ajustar conjuntamente os parâmetros de posição e dispersão segundo a equação (3.12), o estimador robusto para a matriz de covariâncias de $\hat{\boldsymbol{\theta}}$ é dado por

$$
\hat{\mathbf{J}}_{n}^{-1}=\left\{\sum_{i=1}^{n} \hat{\mathbf{S}}_{i}\right\}^{-1}\left\{\sum_{i=1}^{n} \mathbf{M}_{i}^{\top} \hat{\boldsymbol{\Lambda}}_{i} \hat{\mathbf{\Omega}}_{i}^{-1} \hat{\mathbf{b}}_{i} \hat{\mathbf{b}}_{i}^{\top} \hat{\mathbf{\Omega}}_{i}^{-1} \hat{\boldsymbol{\Lambda}}_{i}^{\top} \mathbf{M}_{i}\right\}\left\{\sum_{i=1}^{n} \hat{\mathbf{S}}_{i}\right\}^{-1} .
$$

e o estimador "naive" por $\hat{\mathbf{J}}_{n}^{-1}=-\left\{\sum_{i=1}^{n} \hat{\mathbf{S}}_{i}\right\}^{-1}$, em que $\sum_{i=1}^{n} \mathbf{S}_{i}=-\sum_{i=1}^{n} \mathbf{M}_{i}^{\top} \mathbf{W}_{i} \mathbf{M}_{i}$. As estimativas dessas duas expressões são obtidas substituindo $\boldsymbol{\theta}=\left(\boldsymbol{\beta}^{\top}, \boldsymbol{\gamma}^{\top}\right)^{\top}$ e $\boldsymbol{\alpha}$ por suas respectivas estimativas consistentes.

\subsubsection{Estimação de $\beta, \gamma$ e $\alpha$}

A EEG dada na equação (3.12) expandida em torno de um valor inicial $\hat{\boldsymbol{\theta}}^{(0)}=$ $\left(\hat{\boldsymbol{\beta}}^{(0) \top}, \hat{\boldsymbol{\gamma}}^{(0) \top}\right)^{\top}$ resulta no seguinte processo iterativo para estimar $\boldsymbol{\theta}$ :

$$
\hat{\boldsymbol{\theta}}^{(m+1)}=\hat{\boldsymbol{\theta}}^{(m)}+\left\{\left[\sum_{i=1}^{n} \mathbf{M}_{i}^{\top} \hat{\mathbf{W}}_{i} \mathbf{M}_{i}\right]^{-1}\left[\sum_{i=1}^{n} \mathbf{M}_{i}^{\top} \hat{\mathbf{W}}_{i} \hat{\boldsymbol{\Lambda}}_{i}^{-1} \hat{\mathbf{b}}_{i}\right]\right\}^{(m)}
$$


sendo $m=0,1,2, \ldots$ o número de iterações. O índice $m$ no lado direito das equações acima indica que as matrizes e os vetores são atualizados pelas estimativas de $\boldsymbol{\theta}$ e $\boldsymbol{\alpha}$ da $m$-ésima iteração. Esse processo reescrito na forma de um processo iterativo de mínimos quadrados reponderados é dado por

$$
\hat{\boldsymbol{\theta}}^{(m+1)}=\left\{\left[\sum_{i=1}^{n} \mathbf{M}_{i}^{\top} \hat{\mathbf{W}}_{i} \mathbf{M}_{i}\right]^{-1}\left[\sum_{i=1}^{n} \mathbf{M}_{i}^{\top} \hat{\mathbf{W}}_{i} \mathbf{z}_{i}\right]\right\}^{(m)}
$$

o qual emprega uma matriz de pesos $\mathbf{W}_{i}$ e uma variável dependente modificada $\mathbf{z}_{i}$, sendo $\mathbf{z}_{i}=\hat{\boldsymbol{\nu}}_{i}+\hat{\boldsymbol{\Lambda}}_{i}^{-1} \hat{\mathbf{b}}_{i}, \operatorname{com} \boldsymbol{\nu}_{i}=\left(\boldsymbol{\eta}_{i}^{\top}, \boldsymbol{\delta}_{i}^{\top}\right)^{\top}$.

Para estimar $\boldsymbol{\alpha}$, que reflete as correlações entre $\mathrm{u}_{i j}$ e $\mathrm{u}_{i l}$, para $i=1, \ldots, n$ e $j, l=$ $1, \ldots, t$, com $j \neq l$, procedemos conforme descrito na Seção 2.1.1, mas substituímos o componente $\mathrm{b}_{i j}$ dado na equação (2.8) pelo expresso em (3.7).

A seguir, apresentamos as etapas que mostram como na prática podemos estimar os parâmetros de $\boldsymbol{\beta}, \boldsymbol{\gamma}$ e $\boldsymbol{\alpha}$.

EtAPAS PARA ESTIMAÇÃO DE $\boldsymbol{\beta}, \boldsymbol{\gamma}$ E $\boldsymbol{\alpha}$.

1. Supondo independência entre as observações da mesma unidade experimental, ajustamos um modelo de regressão linear de $g(\mathbf{y})$ sobre $\mathbf{X}$ e um de $f\left(\check{\boldsymbol{\phi}}^{-1}\right)$ sobre $\mathbf{Q}$, ambos via método de mínimos quadrados ordinários, sendo $\boldsymbol{\phi}=\left(\boldsymbol{\phi}_{1}^{\top}, \ldots, \boldsymbol{\phi}_{n}^{\top}\right)^{\top} \mathrm{e}$ $\mathbf{Q}=\left(\mathbf{Q}_{1}^{\top}, \ldots, \mathbf{Q}_{n}^{\top}\right)^{\top}, \operatorname{com} \phi_{i}=\left(\phi_{i 1}, \ldots, \phi_{i t}\right)^{\top}$ e $i=1, \ldots, n$. Para o ajuste do parâmetro de dispersão, utilizamos como valor de $\phi_{i j}^{-1}, \check{\phi}_{i j}^{-1}$, o resultado da equação (1.10), ou seja,

$$
\check{\phi}_{i j}^{-1}=d\left(\mathrm{y}_{i j}, \check{\mu}_{i j}\right)
$$

em que $\check{\mu}_{i j}=g^{-1}\left(\mathbf{x}_{i j}^{\top} \hat{\boldsymbol{\beta}}_{\mathrm{MQO}}\right)$, sendo $\hat{\boldsymbol{\beta}}_{\mathrm{MQO}}$ o vetor de estimativas obtido pelo ajuste da média via método de mínimos quadrados ordinários.

2. Uma estrutura de correlação de trabalho $\mathbf{R}(\boldsymbol{\alpha})$ deve ser definida para ser utilizada na modelagem dos dados. Considerando as estimativas dos parâmetros de regressão da etapa anterior como valores iniciais, $\hat{\boldsymbol{\beta}}^{(0)}$ e $\hat{\boldsymbol{\gamma}}^{(0)}$, obtemos a estimativa inicial de $\boldsymbol{\alpha}, \hat{\boldsymbol{\alpha}}^{(0)}$, levando em conta o componente expresso em (3.7). No passo seguinte do processo iterativo, calculamos $\hat{\boldsymbol{\beta}}^{(m)}$ e $\hat{\boldsymbol{\gamma}}^{(m)}$ pelo processo iterativo dado em (3.13) e atualizamos $\hat{\boldsymbol{\alpha}}^{(m)}$. Repetimos esse passo do processo até a convergência de $\boldsymbol{\beta}$ e $\boldsymbol{\gamma}$. 


\section{Capítulo 4}

\section{Equações de estimação para modelos}

\section{lineares generalizados com medidas repetidas}

Neste capítulo, apresentamos as equações de estimação generalizadas para modelos lineares generalizados com medidas repetidas baseadas nas propostas de Artes (1997), Artes e J $\phi$ rgensen (2000), Liang e Zeger (1986) e Venezuela (2003).

Inicialmente, descrevemos apenas a modelagem do parâmetro de posição, via equações de estimação, considerando a homogeneidade do parâmetro de dispersão. Na Seção 4.2, tanto o parâmetro de posição como o de dispersão são modelados conjuntamente via equações de estimação, com o intuito de incorporar uma possível heterogeneidade da dispersão.

\subsection{Modelagem do parâmetro de posição}

Seja $\mathbf{y}_{i}=\left(\mathrm{y}_{i 1}, \mathrm{y}_{i 2}, \ldots, \mathrm{y}_{i t_{i}}\right)^{\top}$ o vetor de respostas da $i$-ésima unidade experimental, com $i=1, \ldots, n$. Vamos assumir que a densidade marginal de $\mathrm{y}_{i j}$ pertence à família exponencial, isto é, sua densidade (ou função de probabilidade) é dada por

$$
f\left(\mathrm{y}_{i j} ; \varphi_{i j}, \phi\right)=\exp \left\{\phi\left[\mathrm{y}_{i j} \varphi_{i j}-b\left(\varphi_{i j}\right)\right]+c\left(\mathrm{y}_{i j} ; \phi\right)\right\}
$$

sendo $\phi^{-1}>0$ o parâmetro de dispersão considerado conhecido, $b$ e $c$ funções conhecidas. 
Dessa forma, a esperança e a variância de $\mathrm{y}_{i j}$ são dadas por

$$
\mathrm{E}\left(\mathrm{y}_{i j}\right)=\dot{b}\left(\varphi_{i j}\right)=\mu_{i j} \quad \text { e } \quad \operatorname{Var}\left(\mathrm{y}_{i j}\right)=\phi^{-1} \ddot{b}\left(\varphi_{i j}\right)=\phi^{-1} v\left(\mu_{i j}\right)
$$

sendo $\dot{b}\left(\varphi_{i j}\right)$ e $\ddot{b}\left(\varphi_{i j}\right)$, respectivamente, as derivadas de primeira e segunda ordem de $b\left(\varphi_{i j}\right)$ com relação à $\varphi_{i j}$. A função $v\left(\mu_{i j}\right)$ é denominada função de variância, a qual desempenha um papel importante na família exponencial, uma vez que a mesma caracteriza a distribuição. Para facilitar a notação, assumimos, sem perda de generalidade, que $t_{i}=t$, $i=1, \ldots, n$.

As Tabelas C.2 e C.3 do Apêndice C apresentam as distribuições mais importantes pertencentes à família exponencial descrita na equação (4.1).

Para obtermos equações de estimação generalizadas, no contexto de modelos lineares generalizados com medidas repetidas, consideremos que a densidade marginal de $\mathrm{y}_{i j}$ é dada por (4.1), as médias $\mu_{i j}$ 's são modeladas pelo componente sistemático definido em $(2.2)$ e $\mathbf{b}_{1 i}=\mathbf{y}_{i}-\boldsymbol{\mu}_{i}, \operatorname{com} \mathbf{y}_{i}=\left(\mathrm{y}_{i 1}, \ldots, \mathrm{y}_{i t}\right)^{\top}$ e $\boldsymbol{\mu}_{i}=\left(\mu_{i 1}, \ldots, \mu_{i t}\right)^{\top}, i=1, \ldots, n$. Além disso, consideremos $\mathbf{R}(\boldsymbol{\alpha})$ como sendo a matriz de correlação de trabalho (Liang e Zeger, 1986), em que $\boldsymbol{\alpha}$, um vetor $(s \times 1)$, caracteriza completamente $\mathbf{R}(\boldsymbol{\alpha})$. Notemos que $\mathbf{R}(\boldsymbol{\alpha})$ não precisa ser necessariamente a verdadeira matriz de correlação dos $\mathbf{y}_{i}$ 's.

Com isso, a função de estimação generalizada de $\boldsymbol{\beta}$, definida a partir da equação (1.14) e considerando uma matriz de correlação de trabalho, é dada por

$$
\Psi_{1}(\boldsymbol{\beta})=\sum_{i=1}^{n} \mathbf{X}_{i}^{\top} \boldsymbol{\Lambda}_{i} \boldsymbol{\Omega}_{i}^{-1}\left(\mathbf{y}_{i}-\boldsymbol{\mu}_{i}\right)=\sum_{i=1}^{n} \mathbf{X}_{i}^{\top} \mathbf{W}_{i} \boldsymbol{\Lambda}_{i}^{-1} \mathbf{b}_{1 i}
$$

sendo $\mathbf{X}_{i}=\left(\mathbf{x}_{i 1}, \ldots, \mathbf{x}_{i t}\right)^{\top}, \boldsymbol{\Lambda}_{i}=\mathbf{G}_{i}, \boldsymbol{\Omega}_{i}=\phi^{-1} \mathbf{A}_{i}^{1 / 2} \mathbf{R}(\boldsymbol{\alpha}) \mathbf{A}_{i}^{1 / 2}$ e $\mathbf{W}_{i}=\boldsymbol{\Lambda}_{i} \boldsymbol{\Omega}_{i}^{-1} \boldsymbol{\Lambda}_{i}$, com $\mathbf{G}_{i}=\operatorname{diag}\left(\partial g^{-1}\left(\eta_{i 1}\right) / \partial \eta_{i 1}, \ldots, \partial g^{-1}\left(\eta_{i t}\right) / \partial \eta_{i t}\right)$ e $\mathbf{A}_{i}=\operatorname{diag}\left(v\left(\mu_{i 1}\right), \ldots, v\left(\mu_{i t}\right)\right)$. As possíveis expressões para $\mathbf{G}$ de acordo com a função de ligação $g(\cdot)$ adotada estão apresentadas na Tabela C.1 do Apêndice C.

Como a equação de estimação (4.3) não considera a verdadeira matriz de correlação entre as respostas da mesma unidade experimental, deixa de ser uma função de estimação linear ótima. É necessário, então, que um estimador de $\boldsymbol{\alpha}$ seja determinado de modo que o estimador de $\boldsymbol{\beta}$, obtido a partir de (4.3), seja consistente e assintoticamente normal. Na prática, $\phi$ quase sempre é desconhecido, sendo também necessário propor um estimador para esse parâmetro.

Liang e Zeger (1986) descrevem algumas condições que os estimadores de $\boldsymbol{\alpha}$ e $\phi$ devem satisfazer de modo que $\hat{\boldsymbol{\beta}}$, raiz de (4.3), seja consistente e que $\sqrt{n}(\hat{\boldsymbol{\beta}}-\boldsymbol{\beta}) \stackrel{\mathcal{D}}{\longrightarrow} \mathrm{N}_{p}\left(\mathbf{0}, \mathbf{J}^{-1}\right)$, com $\mathbf{J}=\lim _{n \rightarrow \infty} \mathbf{J}_{n} / n$, sendo $\mathbf{J}_{n}$ a matriz de informação de Godambe de $\boldsymbol{\beta}$ associada à 
equação de estimação (4.3), dada por

$$
\mathbf{J}_{n}=\left\{\sum_{i=1}^{n} \mathbf{S}_{i}\right\}\left\{\sum_{i=1}^{n} \mathbf{V}_{i}\right\}^{-1}\left\{\sum_{i=1}^{n} \mathbf{S}_{i}\right\}
$$

em que

$$
\sum_{i=1}^{n} \mathbf{S}_{i}=\mathrm{E}\left[\frac{\partial}{\partial \boldsymbol{\beta}^{\top}} \boldsymbol{\Psi}_{1}(\boldsymbol{\beta})\right]=-\sum_{i=1}^{n} \mathbf{X}_{i}^{\top} \mathbf{W}_{i} \mathbf{X}_{i}
$$

$\mathrm{e}$

$$
\sum_{i=1}^{n} \mathbf{V}_{i}=\mathrm{E}\left[\boldsymbol{\Psi}_{1}(\boldsymbol{\beta}) \Psi_{1}^{\top}(\boldsymbol{\beta})\right]=\sum_{i=1}^{n} \mathbf{X}_{i}^{\top} \boldsymbol{\Lambda}_{i} \boldsymbol{\Omega}_{i}^{-1} \operatorname{Cov}\left(\mathbf{y}_{i}\right) \boldsymbol{\Omega}_{i}^{-1} \boldsymbol{\Lambda}_{i} \mathbf{X}_{i}
$$

Assim, o estimador robusto da matriz de covariâncias de $\hat{\boldsymbol{\beta}}$ é dado por

$$
\hat{\mathbf{J}}_{n}^{-1}=\left\{\sum_{i=1}^{n} \hat{\mathbf{S}}_{i}\right\}^{-1}\left\{\sum_{i=1}^{n} \hat{\mathbf{X}}_{i}^{\top} \hat{\mathbf{G}}_{i} \hat{\mathbf{\Omega}}_{i}^{-1} \hat{\mathbf{b}}_{1 i} \hat{\mathbf{b}}_{1 i}^{\top} \hat{\mathbf{\Omega}}_{i}^{-1} \hat{\mathbf{G}}_{i} \hat{\mathbf{X}}_{i}\right\}\left\{\sum_{i=1}^{n} \hat{\mathbf{S}}_{i}\right\}^{-1},
$$

e o "naive" por $\hat{\mathbf{J}}_{n}^{-1}=-\left\{\sum_{i=1}^{n} \hat{\mathbf{S}}_{i}\right\}^{-1}$. As estimativas dessas duas últimas expressões são obtidas substituindo $\boldsymbol{\beta}, \boldsymbol{\alpha}$ e $\phi$ por suas respectivas estimativas consistentes. Vale enfatizar que esses resultados assintóticos são válidos mesmo quando $\mathbf{R}(\boldsymbol{\alpha})$ não corresponde à verdadeira matriz de correlação de $\mathbf{y}_{i}$.

\subsubsection{Estimação de $\beta, \phi$ e $\alpha$}

Para calcular $\hat{\boldsymbol{\beta}}, \hat{\phi}$ e $\hat{\boldsymbol{\alpha}}$, combinamos o método scoring de Fisher para estimar $\boldsymbol{\beta}$ com o método dos momentos para estimar $\boldsymbol{\alpha}$ e $\phi$. Considerando a equação de estimação (4.3) e as matrizes que a definem, também chegamos ao processo iterativo de mínimos quadrados reponderados para estimar $\boldsymbol{\beta}$, conforme descrito na equação (3.5), o qual emprega uma matriz de pesos $\mathbf{W}_{i}$ e uma variável dependente modificada $\mathbf{z}_{i}$. Esse processo iterativo deve ter as matrizes e os vetores atualizados pelas estimativas de $\boldsymbol{\beta}, \boldsymbol{\alpha}$ e $\phi$ a cada iteração $m$ do processo, com $m=1,2, \ldots$.

A estimativa de $\phi^{-1}$ obtida no $m$-ésimo passo do processo iterativo é dada por

$$
\hat{\phi}^{-(m)}=\left\{\sum_{i=1}^{n} \sum_{j=1}^{t}\left(\frac{\mathrm{y}_{i j}-\hat{\mu}_{i j}^{(m)}}{\sqrt{v\left(\hat{\mu}_{i j}^{(m)}\right)}}\right)^{2} /(n t-p)\right\} .
$$


Aqui, $\boldsymbol{\alpha}$ reflete as correlações entre $\mathrm{y}_{i j}$ e $\mathrm{y}_{i l}$, para $i=1, \ldots, n$ e $j, l=1, \ldots, t$, com $j \neq l$. Logo, para estimar $\boldsymbol{\alpha}$, procedemos conforme descrito na Seção 2.1.1, substituindo $\mathrm{b}_{i j}$ dado na equação (2.8) por

$$
\mathrm{b}_{1 i j}=\mathrm{y}_{i j}-\mu_{i j}
$$

A seguir, apresentamos as etapas que nos mostram como na prática podemos estimar os parâmetros de $\boldsymbol{\beta}, \phi$ e $\boldsymbol{\alpha}$.

EtAPAS PARA ESTIMAÇÃO DE $\boldsymbol{\beta}, \phi$ E $\boldsymbol{\alpha}$.

1. Supondo independência entre as observações da mesma unidade experimental, utilizamos o processo iterativo (3.5) para estimar $\boldsymbol{\beta}$ com $\boldsymbol{\Psi}_{1}(\boldsymbol{\beta})$ conforme dado em (4.3). Esse processo iterativo pode ser iniciado considerando $\hat{\mu}_{i j}^{(0)}=\mathrm{y}_{i j}$ ou, no caso, por exemplo, de uma regressão logística, $\hat{\mu}_{i j}^{(0)}=0,5$. Notemos que a suposição de independência elimina os parâmetros $\boldsymbol{\alpha}$ e $\phi^{-1}$ do processo de estimação de $\boldsymbol{\beta}$.

2. Definimos uma estrutura de correlação de trabalho para ser utilizada na modelagem dos dados. Se a estrutura for a independente, a estimativa de $\boldsymbol{\beta}$ é o vetor de valores obtidos na convergência do processo iterativo da etapa 1. Caso contrário, utilizamos novamente a equação (3.5), considerando como $\hat{\boldsymbol{\beta}}^{(0)}$ a estimativa de $\boldsymbol{\beta}$ encontrada na etapa 1. Estimamos o parâmetro de dispersão $\left(\hat{\phi}^{-(0)}\right)$ e os parâmetros de correlação $\left(\hat{\boldsymbol{\alpha}}^{(0)}\right)$. No passo seguinte do processo iterativo, calculamos os parâmetros de regressão $\left(\hat{\boldsymbol{\beta}}^{(m)}\right)$, o parâmetro de dispersão $\left(\hat{\phi}^{-(m)}\right)$ e os parâmetros de correlação $\left(\hat{\boldsymbol{\alpha}}^{(m)}\right)$. Repetimos esse passo do processo até a convergência.

\subsection{Modelagem conjunta dos parâmetros de posição e dispersão}

Seja $\mathbf{y}_{i}=\left(\mathrm{y}_{i 1}, \mathrm{y}_{i 2}, \ldots, \mathrm{y}_{i t_{i}}\right)^{\top}$ o vetor $\left(t_{i} \times 1\right)$ de respostas da $i$-ésima unidade experimental, com $i=1, \ldots, n$. Vamos assumir que a densidade marginal de $\mathrm{y}_{i j}$ pertence à família exponencial, isto é, sua densidade (ou função de probabilidade) é dada por

$$
f\left(\mathrm{y}_{i j} ; \varphi_{i j}, \phi_{i j}\right)=\exp \left\{\phi_{i j}\left[\mathrm{y}_{i j} \varphi_{i j}-b\left(\varphi_{i j}\right)\right]+c\left(\mathrm{y}_{i j} ; \phi_{i j}\right)\right\} .
$$

Aqui, incorporamos a heterogeneidade do parâmetro de dispersão, ou seja, cada observação $\mathrm{y}_{i j}$ assume ter sua própria dispersão, $\phi_{i j}^{-1}, i=1, \ldots, n$ e $j=1, \ldots, t_{i}$. Para facilitar a notação, assumimos, sem perda de generalidade, que $t_{i}=t, i=1, \ldots, n$. 
No contexto de modelos lineares generalizados com medidas repetidas, consideremos o componente sistemático que modela a média, $\mu_{i j}$, conforme a equação $(2.2)$ e o que modela o parâmetro de dispersão, $\phi_{i j}^{-1}$, conforme a equação (3.8). Além disso, as equações de estimação para estimar a média serão construídas baseadas nos vetores $\mathbf{b}_{1 i}=\mathbf{y}_{i}-\boldsymbol{\mu}_{i}$ (veja justificativa na Seção 4.1); e para estimar a dispersão, serão construídas baseadas nos vetores dados por

$$
\mathbf{b}_{2 i}=\left(\mathrm{b}_{2 i 1}, \ldots, \mathrm{b}_{2 i t}\right)^{\top}
$$

em que $\mathrm{b}_{2 i j}=\left[\mathrm{y}_{i j} \varphi_{i j}-b\left(\varphi_{i j}\right)+\dot{c}\left(\mathrm{y}_{i j} ; \phi_{i j}\right)\right]$ é a função escore para $\phi_{i j}$ obtida a partir da densidade marginal de $\mathrm{y}_{i j}$, sendo $\dot{c}\left(\mathrm{y}_{i j} ; \phi_{i j}\right)$ a derivada de $c\left(\mathrm{y}_{i j} ; \phi_{i j}\right)$ com relação à $\phi_{i j}$, $i=1, \ldots, n$ e $j=1, \ldots, t$. Os vetores descritos em $(4.7), i=1, \ldots, n$, são vetores com média zero sob certas condições de regularidades (Sen e Singer, 1993), mutuamente independentes e satisfazem as mesmas propriedades das funções de estimação regulares definidas na Seção 1.3.

Logo, definindo $\mathbf{b}_{i}=\left(\mathbf{b}_{1 i}^{\top}, \mathbf{b}_{2 i}^{\top}\right)^{\top}$ e assumindo dependência entre as observações da mesma unidade experimental $i$, a função de estimação ótima de $\boldsymbol{\theta}=\left(\boldsymbol{\beta}^{\top}, \boldsymbol{\gamma}^{\top}\right)^{\top}$ definida a partir de (1.14) fica expressa por

$$
\Psi_{2}^{o}(\boldsymbol{\theta})=\sum_{i=1}^{n}\left(\begin{array}{cc}
\mathbf{X}_{i}^{\top} \mathbf{G}_{i} & \mathbf{0} \\
\mathbf{0} & \mathbf{Q}_{i}^{\top} \mathbf{F}_{i} \mathrm{E}\left(\ddot{\mathbf{c}}_{i}\right)
\end{array}\right)\left(\begin{array}{cc}
\operatorname{Cov}\left(\mathbf{b}_{1 i}\right) & \operatorname{Cov}\left(\mathbf{b}_{1 i}, \mathbf{b}_{2 i}\right) \\
\operatorname{Cov}\left(\mathbf{b}_{2 i}, \mathbf{b}_{1 i}\right) & \operatorname{Cov}\left(\mathbf{b}_{2 i}\right)
\end{array}\right)^{-1} \mathbf{b}_{i},
$$

sendo $\mathbf{X}_{i}=\left(\mathbf{x}_{i 1}, \ldots, \mathbf{x}_{i t}\right)^{\top}, \mathbf{G}_{i}=\operatorname{diag}\left(\partial g^{-1}\left(\eta_{i 1}\right) / \partial \eta_{i 1}, \ldots, \partial g^{-1}\left(\eta_{i t}\right) / \partial \eta_{i t}\right), \mathbf{Q}_{i}=\left(\mathbf{q}_{i 1}, \ldots\right.$, $\left.\mathbf{q}_{i t}\right)^{\top}, \mathbf{F}_{i}=\operatorname{diag}\left(\partial f^{-1}\left(\delta_{i 1}\right) / \partial \delta_{i 1}, \ldots, \partial f^{-1}\left(\delta_{i t}\right) / \partial \delta_{i t}\right), \ddot{\mathbf{c}}_{i}=\operatorname{diag}\left(\ddot{c}_{i 1}, \ldots, \ddot{c}_{i t}\right)$ e $\operatorname{Cov}\left(\mathbf{b}_{1 i}\right)=$ $\operatorname{Cov}\left(\mathbf{y}_{i}\right)=\left(\boldsymbol{\Phi}_{i}^{-1} \mathbf{A}_{i}\right)^{1 / 2} \mathbf{R}\left(\mathbf{y}_{i}\right)\left(\mathbf{A}_{i} \boldsymbol{\Phi}_{i}^{-1}\right)^{1 / 2}, \quad$ em que $\boldsymbol{\Phi}_{i}=\operatorname{diag}\left(\phi_{i 1}, \ldots, \phi_{i t}\right), \quad \mathbf{A}_{i}=$ $\operatorname{diag}\left(v\left(\mu_{i 1}\right), \ldots, v\left(\mu_{i t}\right)\right)$ e $\ddot{c}_{i j}$ é a derivada de $\dot{c}\left(\mathrm{y}_{i j} ; \phi_{i j}\right)$ com relação à $\phi_{i j}^{-1}$. Além disso, $\operatorname{Cov}\left(\mathbf{b}_{2 i}\right)$ é a verdadeira matriz de covariâncias de $\mathbf{b}_{2 i}, \operatorname{Cov}\left(\mathbf{b}_{1 i}, \mathbf{b}_{2 i}\right)$ é a verdadeira matriz de covariâncias entre $\mathbf{b}_{1 i}$ e $\mathbf{b}_{2 i}$ e $\mathbf{R}\left(\mathbf{y}_{i}\right)$ é a verdadeira matriz de correlação de $\mathbf{y}_{i}$.

O uso prático das equações descritas em (4.8) traz novas quantidades desconhecidas por modelar conjuntamente os parâmetros de posição e dispersão. Abandonando a propriedade de otimalidade da função de estimação, Artes e J $\phi$ rgensen (2000), que abordam equações de estimação para modelos de dispersão longitudinais, solucionam esse problema atribuindo matrizes de trabalho que deixam $\operatorname{Cov}\left(\mathbf{b}_{1 i}, \mathbf{b}_{2 i}\right)=\mathbf{0}$ e $\operatorname{Cov}\left(\mathbf{b}_{2 i}\right)=\mathbf{I}_{t}$, sendo $\mathbf{I}_{t}$ uma matriz identidade de dimensão $t \times t$. Para o nosso caso que aborda os modelos exponenciais de dispersão, dada a definição de $\mathbf{b}_{2 i}$ em (4.7), iremos aproximar a $\operatorname{Cov}\left(\mathbf{b}_{2 i}\right)$ por $\left\{\boldsymbol{\Pi}_{i} \operatorname{Cov}\left(\mathbf{y}_{i}\right) \boldsymbol{\Pi}_{i}\right\}, \operatorname{com} \boldsymbol{\Pi}_{i}=\operatorname{diag}\left(\varphi_{i 1}, \ldots, \varphi_{i t}\right)$, entendendo que isso fornece um viés inferior do que se considerarmos essa matriz de covariâncias igual a uma matriz identidade. As contas detalhadas estão apresentadas na Seção A.3.1 do Apêndice A e as possíveis 
expressões para $\mathbf{G}$ e $\mathbf{F}$ de acordo, respectivamente, com as funções $g(\cdot)$ e $f(\cdot)$ adotadas estão apresentadas na Tabela C.1 do Apêndice C.

Dessa forma, a função de estimação generalizada de $\boldsymbol{\theta}$ é dada por

$$
\begin{aligned}
\boldsymbol{\Psi}_{2}(\boldsymbol{\theta}) & =\left(\begin{array}{c}
\boldsymbol{\Psi}_{2}(\boldsymbol{\beta}) \\
\boldsymbol{\Psi}_{2}(\boldsymbol{\gamma})
\end{array}\right)=\sum_{i=1}^{n}\left(\begin{array}{c}
\mathbf{X}_{i}^{\top} \mathbf{G}_{i} \boldsymbol{\Sigma}_{i}^{-1} \mathbf{b}_{1 i} \\
\mathbf{Q}_{i}^{\top} \mathbf{F}_{i} \mathrm{E}\left(\ddot{\mathbf{c}}_{i}\right)\left(\boldsymbol{\Pi}_{i} \boldsymbol{\Sigma}_{i} \boldsymbol{\Pi}_{i}\right)^{-1} \mathbf{b}_{2 i}
\end{array}\right)= \\
& =\sum_{i=1}^{n} \mathbf{M}_{i}^{\top} \boldsymbol{\Lambda}_{i} \boldsymbol{\Omega}_{i}^{-1} \mathbf{b}_{i}=\sum_{i=1}^{n} \mathbf{M}_{i}^{\top} \mathbf{W}_{i} \boldsymbol{\Lambda}_{i}^{-1} \mathbf{b}_{i},
\end{aligned}
$$

sendo $\mathbf{M}_{i}=\left(\begin{array}{cc}\mathbf{X}_{i} & \mathbf{0} \\ \mathbf{0} & \mathbf{Q}_{i}\end{array}\right), \boldsymbol{\Lambda}_{i}=\left(\begin{array}{cc}\mathbf{G}_{i} & \mathbf{0} \\ \mathbf{0} & \mathbf{F}_{i} \mathrm{E}\left(\ddot{\mathbf{c}}_{i}\right)\end{array}\right), \boldsymbol{\Sigma}_{i}=\left(\boldsymbol{\Phi}_{i}^{-1} \mathbf{A}_{i}\right)^{1 / 2} \mathbf{R}(\boldsymbol{\alpha})\left(\mathbf{A}_{i} \boldsymbol{\Phi}_{i}^{-1}\right)^{1 / 2}$ $\boldsymbol{\Omega}_{i}=\left(\begin{array}{cc}\boldsymbol{\Sigma}_{i} & \mathbf{0} \\ \mathbf{0} & \boldsymbol{\Pi}_{i} \boldsymbol{\Sigma}_{i} \boldsymbol{\Pi}_{i}\end{array}\right)$ e $\mathbf{W}_{i}=\boldsymbol{\Lambda}_{i} \boldsymbol{\Omega}_{i}^{-1} \boldsymbol{\Lambda}_{i}^{\top}$

A Tabela C.3 do Apêndice C apresenta as funções $\dot{c}$ e $\ddot{c}$ para as distribuições mais importantes pertencentes à família exponencial. Nos casos das distribuições Binomial e de Poisson, o valor de $\phi_{i j}^{-1}$, com $i=1, \ldots, n$ e $j=1, \ldots, t$, é uma constante conhecida e, portanto, a modelagem do parâmetro de dispersão para essas distribuições não se aplica. Ainda vale ressaltar que, para as outras distribuições citadas nessa tabela, a função $\ddot{c}$ não depende da variável resposta simplificando o cálculo de seu valor esperado.

Como a equação de estimação dada em (4.10) deixa de ser a ótima, Artes (1997, Teorema 7, pg.67) descreve algumas condições que o estimador de $\boldsymbol{\alpha}$ deve satisfazer de modo que a raiz de (4.10), $\hat{\boldsymbol{\theta}}$, seja um estimador consistente de $\boldsymbol{\theta}$ e que $\sqrt{n}(\hat{\boldsymbol{\theta}}-$ $\boldsymbol{\theta}) \stackrel{\mathcal{D}}{\longrightarrow} \mathrm{N}_{p+q}\left(\mathbf{0}, \mathbf{J}^{-1}\right)$, com $\mathbf{J}=\lim _{n \rightarrow \infty} \mathbf{J}_{n} / n$, sendo $\mathbf{J}_{n}$ a matriz de informação de Godambe de $\boldsymbol{\theta}$ associada à equação de estimação (4.10).

No caso de ajustar conjuntamente os parâmetros de posição e dispersão segundo a equação (4.10), o estimador robusto para a matriz de covariâncias de $\hat{\boldsymbol{\theta}}$ é dado por

$$
\hat{\mathbf{J}}_{n}^{-1}=\left\{\sum_{i=1}^{n} \hat{\mathbf{S}}_{i}\right\}^{-1}\left\{\sum_{i=1}^{n} \mathbf{M}_{i}^{\top} \hat{\mathbf{\Lambda}}_{i} \hat{\mathbf{\Omega}}_{i}^{-1} \hat{\mathbf{b}}_{i} \hat{\mathbf{b}}_{i}^{\top} \hat{\mathbf{\Omega}}_{i}^{-1} \hat{\boldsymbol{\Lambda}}_{i}^{\top} \mathbf{M}_{i}\right\}\left\{\sum_{i=1}^{n} \hat{\mathbf{S}}_{i}\right\}^{-1} .
$$

e o estimador "naive" por $\hat{\mathbf{J}}_{n}^{-1}=-\left\{\sum_{i=1}^{n} \hat{\mathbf{S}}_{i}\right\}^{-1}$, em que $\sum_{i=1}^{n} \mathbf{S}_{i}=-\sum_{i=1}^{n} \mathbf{M}_{i}^{\top} \mathbf{W}_{i} \mathbf{M}_{i}$. As estimativas dessas duas últimas expressões são obtidas substituindo $\boldsymbol{\theta}=\left(\boldsymbol{\beta}^{\top}, \boldsymbol{\gamma}^{\top}\right)^{\top}$ e $\boldsymbol{\alpha}$ por suas respectivas estimativas consistentes.

Vale ressaltar que, baseada na proposta de McCullagh e Nelder (1989, Capítulo 10), as equações de estimação definidas em (4.8) também poderiam ser construídas utilizando $\mathbf{b}_{2 i}=\mathbf{d}_{i}-\phi_{i}^{-1}$, sendo $\mathbf{d}_{i}=\left(\mathrm{d}_{i 1}, \ldots, \mathrm{d}_{i t}\right)^{\top}$ e $\boldsymbol{\phi}_{i}^{-1}=\left(\phi_{i 1}^{-1}, \ldots, \phi_{i t}^{-1}\right)^{\top}$, com 
$\mathrm{d}_{i j}=\left(\mathrm{y}_{i j}-\mu_{i j}\right)^{2} / v\left(\mu_{i j}\right), i=1, \ldots, n$ e $j=1, \ldots, t$. Entretanto, teríamos necessidade de fazer mais suposições para, na prática, torná-las aplicáveis.

\subsubsection{Estimação de $\beta, \gamma$ e $\alpha$}

Para calcular $\hat{\boldsymbol{\theta}}=\left(\hat{\boldsymbol{\beta}}^{\top}, \hat{\boldsymbol{\gamma}}^{\top}\right)^{\top}$ e $\hat{\boldsymbol{\alpha}}$, combinamos o método scoring de Fisher para estimar $\boldsymbol{\theta}=\left(\boldsymbol{\beta}^{\top}, \boldsymbol{\gamma}^{\top}\right)^{\top}$ com o método dos momentos para estimar $\boldsymbol{\alpha}$. Considerando a equação de estimação (4.10) e as matrizes que a definem, também chegamos ao processo iterativo de mínimos quadrados reponderados para estimar $\boldsymbol{\theta}$, conforme descrito na equação (3.14), o qual emprega uma matriz de pesos $\mathbf{W}_{i}$ e uma variável dependente modificada $\mathbf{z}_{i}$. Esse processo iterativo deve ter as matrizes e os vetores atualizados pelas estimativas de $\boldsymbol{\theta}$ e $\boldsymbol{\alpha}$ a cada passo $m$ do processo, com $m=1,2, \ldots$

Para estimar $\boldsymbol{\alpha}$, que reflete as correlações entre $\mathrm{y}_{i j}$ e $\mathrm{y}_{i l}$, para $i=1, \ldots, n$ e $j, l=$ $1, \ldots, t$, com $j \neq l$, procedemos conforme descrito na Seção 2.1.1, mas substituímos o componente $\mathrm{b}_{i j}$ dado na equação (2.8) pelo expresso em (4.5).

A seguir, apresentamos as etapas que mostram como na prática podemos estimar os parâmetros de $\boldsymbol{\beta}, \boldsymbol{\gamma}$ e $\boldsymbol{\alpha}$.

\section{ETAPAS PARA ESTIMAÇÃO DE $\boldsymbol{\beta}, \boldsymbol{\gamma}$ E $\boldsymbol{\alpha}$.}

1. Supondo independência entre as observações da mesma unidade experimental, ajustamos um modelo de regressão linear de $g(\mathbf{y})$ sobre $\mathbf{X}$ e um de $f\left(\check{\phi}^{-1}\right)$ sobre $\mathbf{Q}$, ambos via método de mínimos quadrados ordinários, sendo $\boldsymbol{\phi}=\left(\boldsymbol{\phi}_{1}^{\top}, \ldots, \boldsymbol{\phi}_{n}^{\top}\right)^{\top} \mathrm{e}$ $\mathbf{Q}=\left(\mathbf{Q}_{1}^{\top}, \ldots, \mathbf{Q}_{n}^{\top}\right)^{\top}, \operatorname{com} \phi_{i}=\left(\phi_{i 1}, \ldots, \phi_{i t}\right)^{\top}$ e $i=1, \ldots, n$. Para o ajuste do parâmetro de dispersão, utilizamos como valor para $\phi_{i j}^{-1}, \check{\phi}_{i j}^{-1}$, a expressão dada por

$$
\check{\phi}_{i j}^{-1}=\left(\mathrm{y}_{i j}-\check{\mu}_{i j}\right)^{2} / v\left(\check{\mu}_{i j}\right)
$$

em que $\check{\mu}_{i j}=g^{-1}\left(\mathbf{x}_{i j}^{\top} \hat{\boldsymbol{\beta}}_{\mathrm{MQO}}\right)$, sendo $\hat{\boldsymbol{\beta}}_{\mathrm{MQO}}$ o vetor de estimativas obtido pelo ajuste da média via método de mínimos quadrados ordinários.

2. Uma estrutura de correlação de trabalho $\mathbf{R}(\boldsymbol{\alpha})$ deve ser definida para ser utilizada na modelagem dos dados. Considerando as estimativas dos parâmetros de regressão da etapa anterior como valores iniciais, $\hat{\boldsymbol{\beta}}^{(0)}$ e $\hat{\boldsymbol{\gamma}}^{(0)}$, obtemos a estimativa inicial de $\boldsymbol{\alpha}, \hat{\boldsymbol{\alpha}}^{(0)}$, levando em conta o componente expresso em (4.5). No passo seguinte do processo iterativo, calculamos $\hat{\boldsymbol{\beta}}^{(m)}$ e $\hat{\boldsymbol{\gamma}}^{(m)}$ pelo processo iterativo dado em (3.13) e atualizamos $\hat{\boldsymbol{\alpha}}^{(m)}$. Repetimos esse passo do processo até a convergência de $\boldsymbol{\beta}$ e $\boldsymbol{\gamma}$. 


\section{Parte II}

\section{Técnicas de Diagnóstico}




\section{Capítulo 5}

\section{Algumas medidas de diagnóstico para dados com medidas repetidas}

A análise de diagnóstico é uma etapa importante para a avaliação do ajuste de um modelo de regressão, pois nos auxilia na verificação de possíveis afastamentos das suposições feitas para o modelo e nos permite detectar observações extremas que interferem desproporcionalmente nos resultados do ajuste.

As técnicas de diagnóstico já se encontram muito bem definidas para os modelos lineares generalizados em Paula (2004) e para modelos lineares generalizados para análise de dados com medidas repetidas em Venezuela et al. (2007). Para esses modelos, os elementos da diagonal principal da matriz de projeção (matriz chapéu), a distância de Cook e os resíduos do modelo ajustado são algumas das medidas existentes na literatura, utilizadas para detectar observações influentes na matriz de covariáveis (pontos alavanca) ou no vetor de respostas e para detectar pontos aberrantes ("outliers").

Com base no trabalho desses autores, na próxima seção, desenvolvemos uma generalização dessas medidas quando abordamos quaisquer equações de estimação definidas tanto para modelagem do parâmetro de posição considerando a homogeneidade do parâmetro de dispersão como para modelagem conjunta dos parâmetros de posição e dispersão. Ambas modelagens consideram dados com medidas repetidas. Na Seção 5.2, apresentamos uma medida baseada no critério de informação de Akaike (Akaike, 1973) para auxiliar na seleção de uma matriz de correlação de trabalho e na seleção de covariáveis para um modelo de regressão. 


\subsection{Pontos alavanca, influentes e aberrantes}

Nesta seção, as medidas de diagnóstico são construídas a partir de um processo iterativo reponderado para estimar parâmetros de regressão $\boldsymbol{\theta}$ definidos para modelagem apenas da média (nesse caso, $\boldsymbol{\theta}=\boldsymbol{\beta}$ ) ou para modelagem conjunta da média e da dispersão (nesse caso, $\boldsymbol{\theta}=\left(\boldsymbol{\beta}^{\top}, \boldsymbol{\gamma}^{\top}\right)^{\top}$ ).

Em outras palavras, dadas as abordagens na primeira parte deste trabalho, aqui os parâmetros de regressão $\boldsymbol{\theta}$ podem ser interpretados de duas formas distintas. Se o interesse for construir medidas de diagnóstico que avaliem apenas o comportamento da modelagem da média, então os parâmetros de regressão $\boldsymbol{\theta}$ são caracterizados pelos parâmetros de regressão $\boldsymbol{\beta}$. E, de acordo com a distribuição adotada sob homogeneidade do parâmetro de dispersão, as medidas de diagnóstico tratadas a seguir são construídas pelos processos iterativos reponderados descritos nas Seções 2.1.1, 3.1.1 e 4.1.1. Mas se o interesse for construir medidas de diagnóstico que avaliem o comportamento conjunto dos ajustes da média e da dispersão dos dados, então os parâmetros de regressão são definidos por $\boldsymbol{\theta}=\left(\boldsymbol{\beta}^{\top}, \boldsymbol{\gamma}^{\top}\right)^{\top}$ e seus processos iterativos reponderados estão descritos nas Seções 2.2.1, 3.2.1 e 4.2.1. Ressaltamos também que as medidas de diagnóstico descritas a seguir serão vetores $(N \times 1)$ quando $\boldsymbol{\theta}=\boldsymbol{\beta}$, com $N=n t$, ou vetores $(2 N \times 1)$ quando $\boldsymbol{\theta}=\left(\boldsymbol{\beta}^{\top}, \boldsymbol{\gamma}^{\top}\right)^{\top}$.

De forma geral, consideremos o processo iterativo reponderado para estimar $\boldsymbol{\theta}$ dado por

$$
\hat{\boldsymbol{\theta}}^{(m+1)}=\left\{\left[\sum_{i=1}^{n} \mathbf{M}_{i}^{\top} \hat{\mathbf{W}}_{i} \mathbf{M}_{i}\right]^{-1}\left[\sum_{i=1}^{n} \mathbf{M}_{i}^{\top} \hat{\mathbf{W}}_{i} \mathbf{z}_{i}\right]\right\}^{(m)}
$$

sendo $\mathbf{M}_{i}=\mathbf{X}_{i}$ quando $\boldsymbol{\theta}=\boldsymbol{\beta}$ ou $\mathbf{M}_{i}=\left(\begin{array}{cc}\mathbf{X}_{i} & \mathbf{0} \\ \mathbf{0} & \mathbf{Q}_{i}\end{array}\right)$ quando $\boldsymbol{\theta}=\left(\boldsymbol{\beta}^{\top}, \boldsymbol{\gamma}^{\top}\right)^{\top}, \hat{\mathbf{W}}_{i}=$ $\hat{\boldsymbol{\Lambda}}_{i} \hat{\boldsymbol{\Omega}}_{i}^{-1} \hat{\boldsymbol{\Lambda}}_{i}$ e $\mathbf{z}_{i}=\hat{\boldsymbol{\nu}}_{i}+\hat{\boldsymbol{\Lambda}}_{i}^{-1} \hat{\mathbf{b}}_{i}$, em que $\hat{\boldsymbol{\nu}}_{i}=\mathbf{M}_{i} \hat{\boldsymbol{\theta}}, i=1, \ldots, n$. As dimensões de $\mathbf{M}_{i}, \mathbf{W}_{i}$ e $\mathbf{z}_{i}$ são, respectivamente, $t^{\prime} \times d, t^{\prime} \times t^{\prime}$ e $t^{\prime} \times 1$, sendo $t^{\prime}=t$ e $d=p$ para o caso que os parâmetros $\boldsymbol{\theta}$ são caracterizados apenas pelos parâmetros $\boldsymbol{\beta}$ (ou seja, $\boldsymbol{\theta}=\boldsymbol{\beta}$ ) ou $t^{\prime}=2 t$ e $d=p+q$ para o caso que $\boldsymbol{\theta}=\left(\boldsymbol{\beta}^{\top}, \boldsymbol{\gamma}^{\top}\right)^{\top}$. Lembramos que $t$ é definido pelo número de observações realizadas em cada unidade experimental $i, p$ é a dimensão de $\boldsymbol{\beta}$ e $q$, a dimensão de $\gamma, \operatorname{com} i=1, \ldots, n$.

Na convergência do processo iterativo para estimar $\boldsymbol{\theta}$ dado em (5.1) e com o intuito de eliminar o somatório para simplificá-lo, temos

$$
\hat{\boldsymbol{\theta}}=\left(\mathbf{M}^{\top} \hat{\mathbf{W}} \mathbf{M}\right)^{-1} \mathbf{M}^{\top} \hat{\mathbf{W}} \mathbf{z}
$$


em que $\mathbf{M}=\left(\mathbf{M}_{1}^{\top}, \ldots, \mathbf{M}_{n}^{\top}\right)^{\top}, \quad \hat{\mathbf{W}}=\operatorname{diag}\left(\hat{\mathbf{W}}_{1}, \ldots, \hat{\mathbf{W}}_{n}\right)$ e $\quad \mathbf{z}=\left(\mathbf{z}_{1}^{\top}, \ldots, \mathbf{z}_{n}^{\top}\right)^{\top}$

Notemos que, em um processo iterativo de mínimos quadrados reponderados, $\mathbf{M}$ representa uma matriz de planejamento (ou de covariáveis), $\hat{\mathbf{W}}$ é uma matriz de pesos e $\mathbf{z}$ desempenha o papel de uma variável dependente modificada. Os dois últimos mudam a cada passo do processo iterativo

\section{PONTO ALAVANCA BASEADO NA MATRIZ DE PROJEÇÃO}

Na equação (5.2), $\hat{\boldsymbol{\theta}}$ pode ser interpretado como a solução de mínimos quadrados da regressão normal linear de $\hat{\mathbf{W}}^{1 / 2} \mathbf{z}$ tendo como matriz de planejamento $\hat{\mathbf{W}}^{1 / 2} \mathbf{M}$. Nessa ótica, o resíduo ordinário, que é a diferença entre os valores observado e ajustado, fica dado por

$$
\mathbf{r}_{O}=\hat{\mathbf{W}}^{1 / 2}(\mathbf{z}-\hat{\boldsymbol{\nu}})=(\mathbf{I}-\mathbf{H}) \hat{\mathbf{W}}^{1 / 2} \mathbf{z}
$$

sendo $\hat{\boldsymbol{\nu}}=\left(\hat{\boldsymbol{\nu}}_{1}^{\top}, \ldots, \hat{\boldsymbol{\nu}}_{n}^{\top}\right)^{\top}$, I a matriz identidade e $\mathbf{H}$ uma matriz bloco diagonal dada por $\mathbf{H}=\operatorname{diag}\left(\mathbf{H}_{1}, \ldots, \mathbf{H}_{n}\right)$, com

$$
\mathbf{H}_{i}=\hat{\mathbf{W}}_{i}^{1 / 2} \mathbf{M}_{i}\left(\mathbf{M}^{\top} \hat{\mathbf{W}} \mathbf{M}\right)^{-1} \mathbf{M}_{i}^{\top} \hat{\mathbf{W}}_{i}^{1 / 2}
$$

tendo dimensão $t^{\prime} \times t^{\prime}$, para todo $i=1, \ldots, n$. A matriz $\mathbf{H}$ é simétrica $\left(\mathbf{H}^{\top}=\mathbf{H}\right) \mathrm{e}$ idempotente $(\mathbf{H H}=\mathbf{H})$.

Como $\hat{\mathbf{W}}^{1 / 2} \mathbf{z}$ faz o papel do vetor resposta, podemos chamar $\mathbf{H}$ de matriz de projeção ortogonal (ou matriz chapéu) como na regressão normal linear em que $\hat{\mathbf{W}}$ é a identidade. Isso nos sugere a utilização dos elementos da diagonal principal de $\mathbf{H}$ para detectarmos a presença de pontos alavanca, assim como Pregibon (1981) fez para os MLGs e Venezuela et al. (2007) fizeram para os MLGs com medidas repetidas.

Dessa forma, a $j$-ésima observação da $i$-ésima unidade experimental pode ser um ponto alavanca no ajuste dos parâmetros $\boldsymbol{\beta}$, se a $\boldsymbol{j}$-ésima observação da diagonal principal de $\mathbf{H}_{i}, \mathrm{~h}_{i j}$, apresentar um valor alto comparado com os valores das demais observações, com $i=1, \ldots, n$ e $j=1, \ldots, t$. Esses resultados podem ser vistos com mais detalhes por meio do gráfico de $\mathrm{h}_{i j}$ versus $i$ que indica a ordem em que cada unidade experimental aparece no conjunto de dados (índice). Quando se trata de uma modelagem conjunta da média e do parâmetro de dispersão, a $j$-ésima observação da $i$-ésima unidade experimental, com $i=1, \ldots, n$ e $j=t+1, \ldots, 2 t$, pode ser um ponto alavanca no ajuste dos parâmetros $\gamma$, se $\mathrm{h}_{i j}$ apresentar um valor alto comparado com os valores das demais observações. Nesse caso, fazemos um gráfico de $\mathrm{h}_{i j}$ versus $i$, sendo $i=1, \ldots, n$ e $j=t+1, \ldots, 2 t$.

Analogamente, a $i$-ésima unidade experimental pode ser um ponto alavanca no ajuste dos parâmetros $\boldsymbol{\beta}$ avaliando $\mathrm{h}_{\beta i}=\sum_{j=1}^{t} \mathrm{~h}_{i j} / t, \operatorname{com} i=1, \ldots, n$. Quando também temos 
o ajuste dos parâmetros $\gamma$, então a $i$-ésima unidade experimental pode ser um ponto alavanca no ajuste desses parâmetros avaliando $\mathrm{h}_{\gamma i \text {. }}=\sum_{j=t+1}^{2 t} \mathrm{~h}_{i j} / t, \operatorname{com} i=1, \ldots, n$. Se o interesse for verificar se a unidade experimental é um ponto alavanca, então fazemos $\mathrm{h}_{\beta i}$. versus $i$ e, quando também ajustamos um modelo para o parâmetro de dispersão,

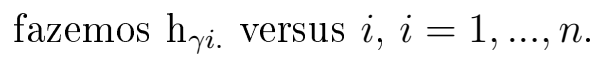

Notemos que os valores de $\mathrm{h}_{i j}$ dependem da matriz de pesos $\hat{\mathbf{W}}_{i}$, portanto observações com valores altos de $\mathrm{h}_{i j}$ nem sempre são pontos alavanca. Ou seja, é necessário examinar com cautela os pontos indicados como alavanca.

\section{PONTO ABERRANTE BASEADO NO RESÍDUO PADRONIZADO}

Se assumirmos que $\operatorname{Cov}(\mathbf{z}) \cong \hat{\mathbf{W}}^{-1}$, então a partir do resíduo ordinário definido em (5.3), temos sua matriz de covariâncias definida por

$$
\begin{aligned}
\operatorname{Cov}\left(\mathbf{r}_{O}\right) & =\operatorname{Cov}\left(\hat{\mathbf{W}}^{1 / 2} \mathbf{z}-\hat{\mathbf{W}}^{1 / 2} \mathbf{M} \hat{\boldsymbol{\theta}}\right)= \\
& =\operatorname{Cov}\left(\hat{\mathbf{W}}^{1 / 2} \mathbf{z}-\hat{\mathbf{W}}^{1 / 2} \mathbf{M}\left(\mathbf{M}^{\top} \hat{\mathbf{W}} \mathbf{M}\right)^{-1} \mathbf{M}^{\top} \hat{\mathbf{W}} \mathbf{z}\right)= \\
& =(\mathbf{I}-\mathbf{H}) \hat{\mathbf{W}}^{1 / 2} \operatorname{Cov}(\mathbf{z}) \hat{\mathbf{W}}^{1 / 2}(\mathbf{I}-\mathbf{H}) \cong(\mathbf{I}-\mathbf{H}) .
\end{aligned}
$$

Como os elementos de $\mathbf{r}_{O}$ possuem variâncias diferentes, o que dificulta compará-los entre si, definimos o resíduo padronizado associado à observação $\mathrm{y}_{i j}$ por

$$
\left(\mathrm{r}_{P D}\right)_{i j}=\frac{\mathbf{e}_{(i j)}^{\top} \hat{\mathbf{W}}^{1 / 2}(\mathbf{z}-\hat{\boldsymbol{\nu}})}{\sqrt{1-\mathrm{h}_{i j}}}=\frac{\mathbf{e}_{(i j)}^{\top} \hat{\mathbf{W}}^{1 / 2} \hat{\boldsymbol{\Lambda}}^{-1} \hat{\mathbf{b}}}{\sqrt{1-\mathrm{h}_{i j}}}
$$

em que $\hat{\boldsymbol{\Lambda}}=\operatorname{diag}\left(\hat{\boldsymbol{\Lambda}}_{1}, \ldots, \hat{\boldsymbol{\Lambda}}_{n}\right)$, $\hat{\mathbf{b}}=\left(\hat{\mathbf{b}}_{1}^{\top}, \ldots, \hat{\mathbf{b}}_{n}^{\top}\right)^{\top}$, $\mathbf{e}_{(i j)}$ é um vetor de tamanho conveniente com valor 1 na posição referente à observação $\mathrm{y}_{i j}$ e 0 nas demais posições e $\mathrm{h}_{i j}$ é o $j$-ésimo elemento da diagonal principal de $\mathbf{H}_{i}$, com $i=1, \ldots, n$ e $j=1, \ldots, t^{\prime}$.

Um ponto aberrante ocorre quando esse possui perfil diferente dos demais no que tange aos valores da variável resposta e também apresenta valor baixo na matriz de projeção H. Com isso, um mesmo ponto dificilmente é alavanca e aberrante.

Para detectá-lo na análise gráfica, podemos utilizar o resíduo padronizado, $\left(\mathrm{r}_{P D}\right)_{i j}$, com $i=1, \ldots, n$ e $j=1, \ldots, t^{\prime}$, versus o índice $i$.

No caso em que todas as respostas são independentes entre si e são modeladas considerando distribuição beta e homogeneidade do parâmetro de dispersão, Espinheira et al. (2008) comparam o resíduo padronizado dado em (5.5) com os resíduos:

$$
\frac{\mathrm{y}_{i j}-\hat{\mu}_{i j}}{\sqrt{\widehat{\operatorname{Var}\left(\mathrm{y}_{i j}\right)}}} \quad \text { e } \quad \frac{\mathrm{y}_{i j}^{*}-\hat{\mu}_{i j}^{*}}{\sqrt{\widehat{\operatorname{Var}\left(\mathrm{y}_{i j}^{*}\right)}}} \text {. }
$$


Esses autores mostram, por meio de dados simulados, que o resíduo padronizado é o único que evidencia claramente um ponto que foi gerado como uma observação aberrante. Espinheira et al. (2008) chamam o resíduo padronizado dado em (5.5) de resíduo reponderado padronizado 2 e os resíduos apresentados em (5.6) são chamados, respectivamente, de resíduo ordinário e resíduo reponderado padronizado 1.

\section{Ponto influente BASEAdo NA DistÂnCia DE CoOK}

Um ponto influente ocorre quando esse possui um perfil diferente dos demais no que tange aos valores da variável resposta, porém apresenta valor alto na matriz de projeção H. Essa influência pode ser detectada pela distância de Cook (Cook, 1977), que mede o afastamento entre a estimativa do vetor paramétrico utilizando todas as observações $(\hat{\boldsymbol{\theta}})$ e sem a observação $\mathrm{y}_{i j}\left(\hat{\boldsymbol{\theta}}_{(i j)}\right), i=1, \ldots, n$ e $j=1, \ldots, t^{\prime}$.

Como, em geral, não é possível obter uma forma fechada para $\hat{\boldsymbol{\theta}}_{(i j)}$, tem sido utilizada uma aproximação de um passo, que consiste em tomar a primeira iteração do processo iterativo pelo método de scoring de Fisher (dado em 5.1) quando o mesmo é iniciado em $\hat{\boldsymbol{\theta}}$. Essa aproximação, introduzida por Pregibon (1981), fica expressa para os modelos de regressão com medidas repetidas na forma:

$$
\hat{\boldsymbol{\theta}}_{(i j)}^{(1)}=\hat{\boldsymbol{\theta}}-\frac{\left[\mathbf{M}^{\top} \hat{\mathbf{W}} \mathbf{M}\right]^{-1}\left[\mathbf{M}^{\top} \hat{\mathbf{W}}^{1 / 2} \mathbf{e}_{(i j)}\right]\left[\mathbf{e}_{(i j)}^{\top} \hat{\mathbf{W}}^{1 / 2} \hat{\boldsymbol{\Lambda}}^{-1} \hat{\mathbf{b}}\right]}{1-\mathrm{h}_{i j}} .
$$

Logo, a distância de Cook, quando se exclui a observação $\mathrm{y}_{i j}$, é definida por

$$
(D C)_{i j}=\frac{1}{d}\left(\hat{\boldsymbol{\theta}}-\hat{\boldsymbol{\theta}}_{(i j)}\right)^{\top} \mathbf{M}^{\top} \hat{\mathbf{W}} \mathbf{M}\left(\hat{\boldsymbol{\theta}}-\hat{\boldsymbol{\theta}}_{(i j)}\right)=\left(\mathrm{r}_{P D}\right)_{i j}^{2} \frac{\mathrm{h}_{i j}}{d\left(1-\mathrm{h}_{i j}\right)} .
$$

Para visualizá-la graficamente, podemos fazer $(D C)_{i j}, i=1, \ldots, n$ e $j=1, \ldots, t^{\prime}$, versus o índice $i$ e indicamos como um ponto influente aquele que possuir um valor alto na distância de Cook quando comparado com os demais pontos. Para o ajuste dos parâmetros $\boldsymbol{\beta}$, a distância de Cook para a unidade experimental $i$ é dada por $(D C)_{\beta i}$. $=\sum_{j=1}^{t}(D C)_{i j} / t$, com $i=1, \ldots, n$. Quando também envolve o ajuste dos parâmetros $\gamma$, a distância de Cook para a unidade experimental $i$ é dada por $(D C)_{\gamma i}=\sum_{j=t+1}^{2 t}(D C)_{i j} / t$, com $i=1, \ldots, n$.

Assim, temos que a distância de Cook detecta pontos influentes sob o efeito da exclusão de cada observação. No Capítulo 6, desenvolvemos medidas de influência local para equações de estimação que detectam pontos influentes sob o efeito de outras pequenas perturbações no modelo ou nos dados.

Como comentário geral, podemos alertar que todas as medidas apresentadas acima podem não ter acurácia se a estimativa da matriz de correlação de trabalho, $\mathbf{R}(\hat{\boldsymbol{\alpha}})$, não for próxima da verdadeira. 


\subsection{Seleção de modelos e de matriz de correlação de trabalho}

O critério de informação de Akaike $(A I C)$ consiste em selecionar o modelo mais parcimonioso entre os ajustados, ou seja, aquele que esteja bem ajustado e com um número reduzido de parâmetros. A construção dessa medida foi motivada por ser um estimador assintoticamente não viesado para a informação de Kullback-Leibler (Kullback e Leibler, 1951) que mede a discrepância entre um modelo candidato e o verdadeiro modelo. Dessa forma, o modelo selecionado dentre aqueles pertencentes ao conjunto de modelos candidatos será o que minimiza a medida $A I C$ dada por

$$
A I C=-2 \ell(\hat{\boldsymbol{\beta}})+2 p,
$$

sendo $\ell(\cdot)$ o logaritmo da função de verossimilhança atribuída aos dados, $\hat{\boldsymbol{\beta}}$ o estimador de máxima verossimilhança com base no modelo candidato e $p$ a dimensão de $\boldsymbol{\beta}$.

Como a construção das equações de estimação generalizadas não são baseadas em verossimilhanças, Pan (2001) propôs uma modificação na medida $A I C$ notando que, quando assumimos independência entre todas as observações, a equação de estimação definida por Liang e Zeger (1986) é equivalente à função quasi-escore (McCullagh e Nelder, 1989) ou, se ativermo-nos à família exponencial, é equivalente à função escore definida para essa família de distribuições. Essa medida proposta por Pan (2001), denominada $Q I C$, é útil tanto para seleção de modelos como para escolha de uma matriz de correlação de trabalho.

Quando temos independência entre todas as observações e supomos homogeneidade da dispersão, as equações de estimação propostas neste trabalho (Parte I) são equivalentes às suas respectivas funções escores. Assim, a medida QIC proposta para selecionar uma matriz de correlação de trabalho $\mathbf{R}$ pode ser escrita, com base no logaritmo da função de verossimilhança, da seguinte forma:

$$
Q I C(\mathbf{R}) \equiv-2 \ell(\hat{\boldsymbol{\beta}}(\mathbf{R}))+2 \operatorname{tr}\left(\hat{\mathbf{S}}_{I} \hat{\mathbf{J}}_{n R}^{-1}\right)
$$

sendo $\ell(\cdot)$ o logaritmo da função de verossimilhança que gera a função escore equivalente à equação de estimação quando assumimos independência entre as observações, $\hat{\mathbf{S}}_{I}$ a matriz de sensibilidade sob a estrutura de independência e $\hat{\mathbf{J}}_{n R}^{-1}$ o estimador robusto sob a estrutura $\mathbf{R}$, os quais são avaliados em $\hat{\boldsymbol{\beta}}(\mathbf{R})$ que é a estimativa de $\boldsymbol{\beta}$ obtida com a matriz de correlação de trabalho $\mathbf{R}$.

Quando todas as especificações da modelagem via equações de estimação estão corretas, $\hat{\mathbf{S}}_{I}$ e $\hat{\mathbf{J}}_{n R}^{-1}$ são assintoticamente equivalentes e $\operatorname{tr}\left(\hat{\mathbf{S}}_{I} \hat{\mathbf{J}}_{n R}^{-1}\right) \approx 2$ (Pan, 2001). Nesse 
caso, a medida $Q I C$ definida na equação (5.8) pode ser reduzida para

$$
Q I C_{s}(\mathbf{R}) \equiv-2 \ell(\hat{\boldsymbol{\beta}}(\mathbf{R}))+2 p
$$

a qual pode ser potencialmente utilizada para seleção de covariáveis.

As medidas acima são válidas quando o parâmetro de dispersão $\phi^{-1}$ é conhecido e único. Quando $\phi^{-1}$ for desconhecido, computamos a medida $Q I C$ utilizando o maior valor estimado para o parâmetro de dispersão dentre os modelos candidatos e a medida $Q I C_{s}$ utilizando o valor estimado para $\phi^{-1}$ obtido do modelo ajustado com todas covariáveis. Isso é feito para que as medidas definidas nas equações (5.8) e (5.9) alterem seus valores por conseqüência apenas da mudança da matriz de correlação de trabalho ou da inclusão/exclusão de covariáveis no modelo. 


\section{Capítulo 6}

\section{Influência local para equações de}

\section{estimação}

A idéia principal de influência local é verificar, por meio de alguma medida apropriada, o efeito de pequenas perturbações no modelo ou nos dados. Se essas perturbações causarem efeitos desproporcionais em determinados resultados do modelo, teremos indícios de que o modelo está mal ajustado ou que existem afastamentos importantes das suposições feitas para o mesmo. A identificação das observações responsáveis por essas discrepâncias pode ajudar na escolha de um modelo mais adequado aos dados.

Cook (1986) propôs um procedimento para avaliar a influência desses pontos examinando o que pequenas perturbações em componentes do modelo causam na superfície do afastamento pela verossimilhança (likelihood displacement) que é definido por

$$
L D \boldsymbol{\omega}=2\left\{\ell(\hat{\boldsymbol{\theta}})-\ell\left(\hat{\boldsymbol{\theta}}_{\omega}\right)\right\},
$$

sendo $\boldsymbol{\omega} \in \mathcal{O} \subseteq \mathcal{R}^{N}$ um vetor de perturbações em geral com dimensão $N \times 1$ com $N=n t$, $\ell(\cdot)$ o logaritmo da função de verossimilhança e $\hat{\boldsymbol{\theta}}$ e $\hat{\boldsymbol{\theta}}_{\omega}$ os respectivos estimadores de máxima verossimilhança de $\boldsymbol{\theta}$ para o modelo postulado e para o modelo perturbado.

Cadigan e Farrell (2002) descrevem essa medida de influência para um caso mais geral, avaliando o afastamento de uma função de ajuste $\mathcal{F}(\boldsymbol{\theta})$ que é duplamente diferenciável em $\boldsymbol{\theta}$ e que tem como estimador de $\boldsymbol{\theta}$, denotado por $\hat{\boldsymbol{\theta}}$, a solução de

$$
\boldsymbol{\Psi}(\hat{\boldsymbol{\theta}})=\left.\left[\frac{\partial \mathcal{F}(\boldsymbol{\theta})}{\partial \boldsymbol{\theta}}\right]\right|_{\boldsymbol{\theta}=\hat{\boldsymbol{\theta}}}=\mathbf{0} .
$$

Dessa forma, a medida de afastamento pela função de ajuste é dada por

$$
F D_{\omega}=2\left\{\mathcal{F}(\hat{\boldsymbol{\theta}})-\mathcal{F}\left(\hat{\boldsymbol{\theta}}_{\omega}\right)\right\}
$$


sendo $\hat{\boldsymbol{\theta}}_{\omega}$ a estimativa que maximiza a função de ajuste perturbada $\mathcal{F}(\boldsymbol{\theta} \mid \boldsymbol{\omega})$. Notemos que quando a função de ajuste é definida pelo logaritmo da função de verossimilhança, temos $F D_{\omega}=L D_{\omega}$. Contudo, $\mathcal{F}$ pode ser alguma outra escolha como, por exemplo, uma função de quasi-verossimilhança, deixando a proposta de Cadigan e Farrell mais geral do que a proposta de Cook.

A seguir, apresentamos a proposta de Cook (1986) escrita na forma mais geral de Cadigan e Farrell (2002), denominada influência local generalizada. Na Seção 6.2, desenvolvemos uma medida de influência local para equações de estimação com base nas propostas apresentadas pelos autores citados anteriormente.

\subsection{Influência local generalizada}

Conforme visto anteriormente, a medida $F D_{\omega}$ pode ser utilizada para avaliar a influência sobre a estimativa de $\boldsymbol{\theta}$ ao se variar $\boldsymbol{\omega}$ em $\mathcal{O}$. No entanto, avaliar o comportamento de $F D_{\omega}$ para todo $\boldsymbol{\omega} \in \mathcal{O}$ pode ser inviável devido à infinidade de valores que $\boldsymbol{\omega}$ pode assumir. Dessa forma, Cook (1986) propõe estudar o comportamento local de $F D_{\omega}$ para qualquer valor de $\boldsymbol{\omega}$ em uma vizinhança de $\boldsymbol{\omega}_{0}$ que representa ausência de perturbação, ou seja, $\boldsymbol{\omega}_{0}$ é tal que $\mathcal{F}\left(\boldsymbol{\theta} \mid \boldsymbol{\omega}_{0}\right)=\mathcal{F}(\boldsymbol{\theta})$ ou, analogamente, $F D_{\omega_{0}}=0$. A sugestão desse autor é investigar a curvatura normal (Bates e Watts, 1980) da linha projetada no gráfico $F D_{\omega_{0}+a d} \times a$, em que $a \in \mathcal{R}$ e $d$ é uma direção arbitrária de norma igual a um, ou seja, $\|d\|=1$. Essa curvatura normal pode ser expressa numa forma geral dada por $C_{d}(\boldsymbol{\theta})=2\left|d^{\top} \boldsymbol{\Delta}^{\top} \ddot{\mathcal{F}}^{-1} \boldsymbol{\Delta} d\right|$, sendo

$$
\boldsymbol{\Delta}=\frac{\partial^{2} \mathcal{F}(\boldsymbol{\theta} \mid \boldsymbol{\omega})}{\partial \boldsymbol{\theta} \partial \boldsymbol{\omega}^{\top}}=\frac{\partial \boldsymbol{\Psi}(\boldsymbol{\theta} \mid \boldsymbol{\omega})}{\partial \boldsymbol{\omega}^{\top}}
$$

e

$$
\ddot{\mathcal{F}}=\frac{\partial^{2} \mathcal{F}(\boldsymbol{\theta})}{\partial \boldsymbol{\theta} \partial \boldsymbol{\theta}^{\top}}=\frac{\partial \boldsymbol{\Psi}(\boldsymbol{\theta})}{\partial \boldsymbol{\theta}^{\top}}
$$

com todas as quantidades avaliadas em $\boldsymbol{\theta}=\hat{\boldsymbol{\theta}}$ e $\boldsymbol{\omega}=\boldsymbol{\omega}_{0}$, em que $\boldsymbol{\Psi}(\cdot \mid \cdot)$ é o vetor gradiente da função de ajuste $\mathcal{F}(\cdot \mid \cdot)$. Para identificar as observações que, sob pequenas perturbações, exercem notável influência local em $F D_{\omega_{0}}$, vamos inspecionar a direção do autovetor $d_{\text {max }}$ correspondente à linha projetada de maior curvatura $C_{\max }$ que é obtida pelo maior autovalor da matriz

$$
-\Delta^{\top} \ddot{\mathcal{F}}^{-1} \Delta
$$

Ospina (2007, Apêndice 3.A.1) apresenta ilustrações que facilitam entender a idéia de Cook (1986) ao definir a medida de influência local descrita em (6.4). 
Além de identificar quais observações influenciam conjuntamente na estimação do vetor de parâmetros $\boldsymbol{\theta}$, também é possível avaliar a influência local apenas para parte de $\boldsymbol{\theta}$, assumindo que esse vetor é particionado na forma $\boldsymbol{\theta}=\left(\boldsymbol{\theta}_{1}^{\top}, \boldsymbol{\theta}_{2}^{\top}\right)^{\top}$. Nesse caso, se o interesse é identificar observações influentes somente na estimação de $\boldsymbol{\theta}_{1}$, a curvatura normal na direção $d$ é dada por $C_{d}\left(\boldsymbol{\theta}_{1}\right)=2\left|d^{\top} \boldsymbol{\Delta}^{\top}\left(\ddot{\mathcal{F}}^{-1}-\ddot{\mathcal{F}}^{\theta_{2} \theta_{2}}\right) \boldsymbol{\Delta} d\right|$, em que

$$
\ddot{\mathcal{F}}^{\theta_{2} \theta_{2}}=\left[\begin{array}{cc}
\mathbf{0} & \mathbf{0} \\
\mathbf{0} & \ddot{\mathcal{F}}_{\theta_{2} \theta_{2}}^{-1}
\end{array}\right],
$$

com $\ddot{\mathcal{F}}_{\theta_{2} \theta_{2}}$ avaliada em $\hat{\boldsymbol{\theta}}$. O gráfico de índices de $d_{\text {max }}$ da matriz $-\boldsymbol{\Delta}^{\top}\left(\ddot{\mathcal{F}}^{-1}-\ddot{\mathcal{F}}^{\theta_{2} \theta_{2}}\right) \boldsymbol{\Delta}$ pode revelar quais observações são influentes em $\hat{\boldsymbol{\theta}}_{1}$. Similarmente, a curvatura normal para o parâmetro $\boldsymbol{\theta}_{2}$ na direção $d$ é dada por $C_{d}\left(\boldsymbol{\theta}_{2}\right)=2\left|d^{\top} \boldsymbol{\Delta}^{\top}\left(\ddot{\mathcal{F}}^{-1}-\ddot{\mathcal{F}}^{\theta_{1} \theta_{1}}\right) \boldsymbol{\Delta} d\right|$, em que

$$
\ddot{\mathcal{F}}^{\theta_{1} \theta_{1}}=\left[\begin{array}{cc}
\ddot{\mathcal{F}}_{\theta_{1} \theta_{1}}^{-1} & \mathbf{0} \\
\mathbf{0} & \mathbf{0}
\end{array}\right],
$$

com $\ddot{\mathcal{F}}_{\theta_{1} \theta_{1}}$ avaliada em $\hat{\boldsymbol{\theta}}$. A influência local das observações em $\hat{\boldsymbol{\theta}}_{2}$ pode ser avaliada se considerarmos o gráfico de índices do maior autovetor $d_{\text {max }}$ da matriz $-\Delta^{\top}\left(\ddot{\mathcal{F}}^{-1}-\ddot{\mathcal{F}}^{\theta_{1} \theta_{1}}\right) \boldsymbol{\Delta}$

\subsection{Influência local para equações de estimação}

Na Seção 6.1, vimos que a medida de influência local generalizada pode ser construída a partir de qualquer função de ajuste $\mathcal{F}(\boldsymbol{\theta})$ desde que essa exista e satisfaça (6.1). É fácil ver que essa medida de influência também pode ser construída a partir do seu vetor gradiente $\boldsymbol{\Psi}(\boldsymbol{\theta})$ sem necessariamente conhecer a função de ajuste $\mathcal{F}(\boldsymbol{\theta})$ que o gera, desde que garantimos a sua existência e que a mesma satisfaça (6.1).

No contexto de equação de estimação generalizada, não conhecemos a função de verossimilhança ou a função de ajuste que gera essa equação. Entretanto, vamos garantir a sua existência assumindo que qualquer equação de estimação generalizada, que utiliza a verdadeira matriz de correlação quando construída a partir de (1.14) ou que utiliza uma matriz de correlação de trabalho $\mathbf{R}(\boldsymbol{\alpha})$ conhecida, satisfaz as propriedades de quasiverossimilhança citadas por McCullagh e Nelder (1989, Seção 9.3.2).

Esses autores descrevem que uma função quasi-escore com observações dependentes é um vetor gradiente de uma quasi-verossimilhança, desde que a derivada dessa função quasi-escore com relação à $\boldsymbol{\beta}$ seja uma matriz simétrica ou, similarmente, desde que as 
derivadas dos componentes de $\operatorname{Cov}\left(\mathbf{y}_{i}\right)^{-1}$ com respeito à $\boldsymbol{\mu}_{i}$ sejam iguais sob a permutação de três índices. Em outras palavras, desde que satisfaça

$$
\frac{\partial \operatorname{Cov}\left(\mathrm{y}_{i j}, \mathrm{y}_{i l}\right)^{-1}}{\partial \mu_{i k}}=\frac{\partial \operatorname{Cov}\left(\mathrm{y}_{i j}, \mathrm{y}_{i k}\right)^{-1}}{\partial \mu_{i l}}=\frac{\partial \operatorname{Cov}\left(\mathrm{y}_{i l}, \mathrm{y}_{i k}\right)^{-1}}{\partial \mu_{i j}},
$$

com $i=1, \ldots, n$ e $j, l, k=1, \ldots, t$. Transferindo essas propriedades ao nosso caso, verificamos que as derivadas dos componentes da matriz $\operatorname{Cov}\left(\mathbf{b}_{i}\right)^{-1}$ descrita em (1.15) com respeito à $\boldsymbol{\mu}_{i}$ são iguais sob a permutação dos três índices $j, l$ e $k, \operatorname{com} i=1, \ldots, n$ e $j, l, k=1, \ldots, t$. Isso acontece quando utilizamos a verdadeira matriz de correlação $\mathbf{R}\left(\mathbf{b}_{i}\right)$ ou quando utilizamos uma matriz de correlação de trabalho $\mathbf{R}(\boldsymbol{\alpha})$ conhecida, já que ambas não dependem de $\boldsymbol{\mu}$.

Logo, garantimos que qualquer equação de estimação $\Psi(\boldsymbol{\theta})$ que utiliza a verdadeira matriz de correlação ou uma matriz de correlação de trabalho conhecida é um vetor gradiente de uma função de ajuste $\mathcal{F}(\boldsymbol{\theta})$. Isto é, podemos assumir que

$$
\exists \mathcal{F}(\boldsymbol{\theta}) \quad \text { tal que } \quad \frac{\partial \mathcal{F}(\boldsymbol{\theta})}{\partial \boldsymbol{\theta}}=\boldsymbol{\Psi}(\boldsymbol{\theta}) \quad \text { e } \quad \boldsymbol{\Psi}(\hat{\boldsymbol{\theta}})=\mathbf{0},
$$

em que $\hat{\boldsymbol{\theta}}$ é o ponto de máximo da função de ajuste $\mathcal{F}(\boldsymbol{\theta})$. Dessa forma, podemos utilizar a matriz $\boldsymbol{\Delta}$ definida na equação (6.2) para obter o autovetor $d_{\max }$ a partir da equação (6.4). Já a matriz hessiana $\ddot{\mathcal{F}}$ definida em (6.3) nem sempre é facilmente obtida para alguns modelos, mas a mesma pode ser simplificada utilizando seu respectivo valor esperado (Cadigan, 1995). No nosso caso, o valor esperado de $\ddot{\mathcal{F}}$ é dado pela matriz de sensibilidade conforme definição em (1.12).

Assim, considerando que a equação de estimação $\boldsymbol{\Psi}(\boldsymbol{\theta})$ é um vetor gradiente de uma função de ajuste, ainda que desconhecida, e satisfaz (6.1), propomos uma medida de influência local para equação de estimação definida pelo autovetor $d_{\text {max }}$ correspondente ao maior autovalor da matriz

$$
-\Delta^{\top} \mathbf{S}^{-1} \boldsymbol{\Delta}
$$

sendo

$$
\boldsymbol{\Delta}=\frac{\partial \boldsymbol{\Psi}(\boldsymbol{\theta} \mid \boldsymbol{\omega})}{\partial \boldsymbol{\omega}^{\top}} \quad \text { e } \quad \mathbf{S}=\mathrm{E}(\ddot{\mathcal{F}})=\mathrm{E}\left(\frac{\partial \boldsymbol{\Psi}(\boldsymbol{\theta})}{\partial \boldsymbol{\theta}^{\top}}\right)
$$

avaliadas em $\boldsymbol{\theta}=\hat{\boldsymbol{\theta}}$ e $\boldsymbol{\omega}=\boldsymbol{\omega}_{0}$.

Ressaltamos que pela notação utilizada na primeira parte deste trabalho, quando estamos modelando a média sob homogeneidade do parâmetro de dispersão, o vetor de parâmetros $\boldsymbol{\theta}$ representa apenas os parâmetros de regressão $\boldsymbol{\beta}$. Assim, o gráfico de índices 
de $d_{\text {max }}$ pode revelar quais observações são influentes na estimação de $\boldsymbol{\beta}$. Quando temos uma modelagem conjunta da média e da dispersão, $d_{\text {max }}$ pode revelar quais observações são influentes na estimação de $\boldsymbol{\theta}=\left(\boldsymbol{\beta}^{\top}, \boldsymbol{\gamma}^{\top}\right)^{\top}$. Nesse caso, se o interesse é identificar observações influentes somente na estimação dos parâmetros $\boldsymbol{\beta}$ ou $\boldsymbol{\gamma}$, podemos utilizar o conceito de partição de $\boldsymbol{\theta}$ conforme apresentado na Seção 6.1.

Logo, para identificar observações influentes somente na estimação de $\boldsymbol{\beta}$, a curvatura normal na direção $d$ é dada por $C_{d}(\boldsymbol{\beta})=2\left|d^{\top} \boldsymbol{\Delta}^{\top}\left(\mathbf{S}^{-1}-\mathbf{S}^{\gamma \gamma}\right) \boldsymbol{\Delta} d\right|$, em que

$$
\mathbf{S}^{\gamma \gamma}=\left[\begin{array}{cc}
\mathbf{0} & \mathbf{0} \\
\mathbf{0} & \mathbf{S}_{\gamma \gamma}^{-1}
\end{array}\right],
$$

com $\mathbf{S}_{\gamma \gamma}$ avaliada em $\hat{\boldsymbol{\theta}}$. O gráfico de índices do maior autovetor de $-\boldsymbol{\Delta}^{\top}\left(\mathbf{S}^{-1}-\mathbf{S}^{\gamma \gamma}\right) \boldsymbol{\Delta}$ pode revelar quais observações são influentes na estimação de $\boldsymbol{\beta}$.

Similarmente, a curvatura normal para os parâmetros de regressão $\gamma$ na direção $d$ é dada por $C_{d}\left(\boldsymbol{\theta}_{2}\right)=2\left|d^{\top} \boldsymbol{\Delta}^{\top}\left(\mathbf{S}^{-1}-\mathbf{S}^{\beta \beta}\right) \boldsymbol{\Delta} d\right|$, em que

$$
\mathbf{S}^{\beta \beta}=\left[\begin{array}{cc}
\mathbf{S}_{\beta \beta}^{-1} & \mathbf{0} \\
\mathbf{0} & \mathbf{0}
\end{array}\right] \text {, }
$$

$\operatorname{com} \mathbf{S}_{\beta \beta}$ avaliada em $\hat{\boldsymbol{\theta}}$. A influência local das observações em $\hat{\gamma}$ pode ser avaliada se considerarmos o gráfico de índices do maior autovetor $d_{\max }$ da matriz $-\boldsymbol{\Delta}^{\top}\left(\mathbf{S}^{-1}-\mathbf{S}^{\beta \beta}\right) \boldsymbol{\Delta}$.

A seguir, apresentamos as medidas de influência local para alguns esquemas de perturbação considerando modelos de regressão beta, modelos de regressão simplex e modelos lineares generalizados, todos com medidas repetidas conforme descritos, respectivamente, nos Capítulos 2, 3 e 4. Essas medidas são desenvolvidas para os esquemas de perturbação ponderação de casos, da variável resposta, de uma covariável contínua da matriz de planejamento, no parâmetro de precisão e, finalmente, na matriz de correlação de trabalho. Todos esses esquemas são tratados para modelagem da média sob homogeneidade do parâmetro de dispersão e os três primeiros, para modelagem conjunta da média e do parâmetro de dispersão o qual considera dispersão variável.

Para identificar possíveis observações influentes na estimação dos parâmetros de regressão ou para auxiliar na indicação de um modelo mais adequado aos dados, graficamente, podemos avaliar o comportamento das medidas de influência local de diversas formas. A ponderação de casos pode ser interpretada como uma perturbação na variância de cada unidade experimental, em especial para modelos normais lineares (Thomas e Cook, 1989). Cook (1986), baseado nesse tipo de perturbação, conclui para dois exemplos de regressão linear simples que os gráficos de $d_{\max }$ versus valores de covariáveis evidenciam a heteroscedasticidade dos dados auxiliando a identificar quais covariáveis devem ser usadas para a modelagem da variância. 
Com o esquema de perturbação individual de cada covariável, é possível avaliar sua influência no processo de estimação do modelo. No entanto, esse tipo de perturbação faz sentido apenas se a covariável é medida de forma contínua. A perturbação no parâmetro de precisão avalia o quão sensível os dados se mostram em relação à uma perturbação heteroscedástica. O gráfico de índices de $d_{\max }$ obtido com esse esquema de perturbação pode revelar quais casos contribuem mais para essa possível sensibilidade. Adicionalmente, o gráfico de $d_{\max }$ versus o valor predito pode indicar a necessidade da modelagem do parâmetro de dispersão, se esse apresentar, por exemplo, um comportamento de uma função exponencial. Finalmente, o esquema de perturbação na matriz de correlação de trabalho $\mathbf{R}(\boldsymbol{\alpha})$, que assumimos ser a mesma para todas as unidades experimentais, pode indicar a necessidade, por exemplo, de impor matrizes de trabalho distintas para grupos de unidade experimentais. Graficamente, podemos avaliar a medida $d_{\text {max }}$ versus os índices das unidades experimentais ordenados por grupo.

\subsection{Esquemas de perturbação sob homogeneidade da dispersão}

As equações de estimação generalizadas para modelagem da média sob suposição de homogeneidade do parâmetro de dispersão estão definidas nas equações (2.4), (3.3) e (4.3) para, respectivamente, modelos de regressão beta, modelos de regressão simplex e modelos lineares generalizados. De forma geral, essas equações de estimação podem ser expressas por

$$
\Psi_{1}(\boldsymbol{\beta})=\mathbf{X}^{\top} \boldsymbol{\Lambda} \boldsymbol{\Omega}^{-1} \mathbf{b}=\mathbf{X}^{\top} \mathbf{W} \Lambda^{-1} \mathbf{b}
$$

cuja matriz de sensibilidade, conforme definição em (1.12), é dada por:

$$
\mathbf{S}=-\mathbf{X}^{\top} \mathbf{W X}
$$

em que $\mathbf{X}=\left(\mathbf{X}_{1}^{\top}, \ldots, \mathbf{X}_{n}^{\top}\right)^{\top}, \boldsymbol{\Lambda}=\operatorname{diag}\left(\boldsymbol{\Lambda}_{1}, \ldots, \boldsymbol{\Lambda}_{n}\right), \quad \boldsymbol{\Omega}=\operatorname{diag}\left(\boldsymbol{\Omega}_{1}, \ldots, \boldsymbol{\Omega}_{n}\right), \mathbf{W}=$ $\operatorname{diag}\left(\mathbf{W}_{1}, \ldots, \mathbf{W}_{n}\right)$ e $\mathbf{b}=\left(\mathbf{b}_{1}^{\top}, \ldots, \mathbf{b}_{n}^{\top}\right)^{\top}$.

Assim, as medidas de influência local descritas a seguir para alguns esquemas de perturbação sob homogeneidade da dispersão serão definidas a partir das equações (6.6) e (6.7), avaliadas sob as estimativas do modelo postulado $\left(\hat{\boldsymbol{\beta}}^{\top}, \hat{\phi}\right)^{\top}$ e em $\boldsymbol{\omega}_{0}$. 


\subsubsection{Ponderação de casos}

Considere o esquema de perturbação

$$
\boldsymbol{\Psi}_{1}(\boldsymbol{\beta} \mid \boldsymbol{\omega})=\mathbf{X}^{\top} \mathbf{W} \boldsymbol{\Lambda}^{-1} \operatorname{diag}(\boldsymbol{\omega}) \mathbf{b}
$$

em que $\boldsymbol{\omega}=\left(\boldsymbol{\omega}_{1}^{\top}, \ldots, \boldsymbol{\omega}_{n}^{\top}\right)^{\top}$, com $\boldsymbol{\omega}_{i}=\left(\omega_{i 1}, \ldots, \omega_{i t}\right)^{\top}, i=1, \ldots, n$. Aqui, o vetor que representa ausência de perturbação, $\boldsymbol{\omega}_{0}$, assume $\omega_{i j}=1$, com $i=1, \ldots, n$ e $j=1, \ldots, t$.

Para a perturbação definida em (6.8), é fácil ver que $\boldsymbol{\Delta}=\mathbf{X}^{\top} \mathbf{W} \boldsymbol{\Lambda}^{-1} \operatorname{diag}(\mathbf{b})$. Neste caso, a matriz definida em (6.5) é dada por

$$
\operatorname{diag}(\mathbf{b}) \Lambda^{-1} \mathbf{W}^{\top} \mathbf{X}\left(\mathbf{X}^{\top} \mathbf{W} \mathbf{X}\right)^{-1} \mathbf{X}^{\top} \mathbf{W} \Lambda^{-1} \operatorname{diag}(\mathbf{b}),
$$

avaliada em $\boldsymbol{\omega}_{0}$ e em $\left(\hat{\boldsymbol{\beta}}^{\top}, \hat{\phi}\right)^{\top}$,

Quando a equação (6.8) é definida a partir da equação de estimação (2.4) e temos todas as observações independentes entre si, a matriz $\boldsymbol{\Delta}$ descrita para ponderação de casos coincide com a apresentada por Ospina (2007, Seção 3.4.1).

\subsubsection{Perturbação da variável resposta}

Considere um esquema aditivo de perturbação na variável resposta $\mathrm{y}_{i j}$ dado por

$$
\mathrm{y}_{\omega i j}=\mathrm{y}_{i j}+\omega_{i j} \sqrt{\operatorname{Var}\left(\mathrm{y}_{i j}\right)}
$$

em que $\omega_{i j}=0$ equivale à ausência de perturbação, ou seja, $\boldsymbol{\omega}_{0}=\mathbf{0}$.

Analisando a equação de estimação definida em (6.6), vemos que a única parte que depende da variável resposta está no vetor $\mathbf{b}$. Assim, considerando $\mathbf{b}_{\omega}$ o vetor $\mathbf{b}$ com perturbação na variável resposta, a equação de estimação perturbada fica escrita na forma

$$
\Psi_{1}(\boldsymbol{\beta} \mid \boldsymbol{\omega})=\mathbf{X}^{\top} \mathbf{W} \Lambda^{-1} \mathbf{b}_{\omega}
$$

em que $\mathbf{b}_{\omega}=\left(\mathbf{b}_{\omega 1}^{\top}, \ldots, \mathbf{b}_{\omega n}^{\top}\right)^{\top}$, com $\mathbf{b}_{\omega i}=\left(\mathrm{b}_{\omega i 1}, \ldots, \mathrm{b}_{\omega i t}\right)^{\top}, i=1, \ldots, n$.

Nesse caso, a matriz definida em (6.2) fica expressa por $\boldsymbol{\Delta}=\mathbf{X}^{\top} \mathbf{W} \boldsymbol{\Lambda}^{-1} \mathcal{B}$, sendo

$$
\mathcal{B}=\frac{\partial \mathbf{b}_{\omega}}{\partial \boldsymbol{\omega}^{\top}}
$$

Logo, com base na equação (6.5), a medida de influência local com perturbação da variável resposta é obtida da matriz

$$
\mathcal{B}^{\top} \Lambda^{-1} \mathbf{W}^{\top} \mathbf{X}\left(\mathbf{X}^{\top} \mathbf{W} \mathbf{X}\right)^{-1} \mathbf{X}^{\top} \mathbf{W} \Lambda^{-1} \mathcal{B},
$$


avaliada em $\boldsymbol{\omega}_{0}$ e em $\left(\hat{\boldsymbol{\beta}}^{\top}, \hat{\phi}\right)^{\top}$

Nas equações (2.4), (3.3) e (4.3), os vetores b's são definidos de formas diferentes ocasionando medidas de influência local distintas para cada caso. A seguir, para cada uma dessas três equações de estimação, apresentamos a derivada do vetor perturbado $\mathbf{b}_{\omega}$ com relação ao vetor de perturbação $\boldsymbol{\omega}$.

\section{BETA}

De acordo com a equação de estimação definida em (2.4), o vetor perturbado na variável resposta referente à $i$-ésima unidade experimental $\mathbf{b}_{\omega i}$ é dado por

$$
\mathbf{b}_{\omega i}=\mathbf{y}_{\omega i}^{*}-\boldsymbol{\mu}_{i}^{*}
$$

em que $\mathbf{y}_{\omega i}^{*}=\left(\mathrm{y}_{\omega i 1}^{*}, \ldots, \mathrm{y}_{\omega i t}^{*}\right)^{\top}$, com $\mathrm{y}_{\omega i j}^{*}=\log \left(\frac{\mathrm{y}_{\omega i j}}{1-\mathrm{y}_{\omega i j}}\right), i=1, \ldots, n$ e $j=1, \ldots, t$. Assim, da Seção B.1.1 do Apêndice B, obtemos

$$
\frac{\partial \mathrm{b}_{\omega i j}}{\partial \omega_{i j}}=\frac{s_{i j}}{v\left(\mathrm{y}_{\omega i j}\right)},
$$

sendo $s_{i j}=\sqrt{v\left(\mu_{i j}\right) /(1+\phi)}$ e $v\left(\mathrm{y}_{\omega i j}\right)=\mathrm{y}_{\omega i j}\left(1-\mathrm{y}_{\omega i j}\right), \operatorname{com} i=1, \ldots, n$ e $j=1, \ldots, t$. Com isso, temos

$$
\mathcal{B}=\mathcal{S} \Upsilon^{-1}
$$

sendo $\boldsymbol{\Upsilon}=\operatorname{diag}\left(\boldsymbol{\Upsilon}_{1}, \ldots, \boldsymbol{\Upsilon}_{n}\right)$ e $\mathcal{S}=\operatorname{diag}\left(\mathcal{S}_{1}, \ldots, \mathcal{S}_{n}\right)$, em que $\boldsymbol{\Upsilon}_{i}=\operatorname{diag}\left(v\left(\mathrm{y}_{\omega i 1}\right), \ldots\right.$, $\left.v\left(\mathrm{y}_{\omega i t}\right)\right)$ e $\mathcal{S}_{i}=\operatorname{diag}\left(s_{i 1}, \ldots, s_{i t}\right), \operatorname{com} i=1, \ldots, n$. Notemos que $v\left(\mathrm{y}_{\omega i j}\right)$ avaliado em $\boldsymbol{\omega}_{0}$ é dado por $v\left(\mathrm{y}_{i j}\right)=\mathrm{y}_{i j}\left(1-\mathrm{y}_{i j}\right)$, com $i=1, \ldots, n$ e $j=1, \ldots, t$.

No caso em que todas as observações são independentes entre si, nossa matriz $\boldsymbol{\Delta}$ definida para perturbação da variável resposta coincide com a mesma matriz apresentada por Ospina (2007, Seção 3.4.2).

\section{SIMPLEX}

Considerando a equação de estimação (3.3), a $j$-ésima observação do vetor perturbado na variável resposta referente à $i$-ésima unidade experimental, $\mathrm{b}_{\omega i j}$, é dada por

$$
\mathrm{b}_{\omega i j}=-\frac{1}{2} \frac{\partial d\left(\mathrm{y}_{\omega i j} ; \mu_{i j}\right)}{\partial \mu_{i j}}=-\frac{\mathrm{y}_{\omega i j}-\mu_{i j}}{2 \mu_{i j}\left(1-\mu_{i j}\right)}\left\{d\left(\mathrm{y}_{\omega i j} ; \mu_{i j}\right)+\frac{1}{\mu_{i j}^{2}\left(1-\mu_{i j}\right)^{2}}\right\}
$$

com $i=1, \ldots, n$ e $j=1, \ldots, t$. Assim, da Seção B.1.1 do Apêndice B, calculamos que

$$
\frac{\partial \mathrm{b}_{\omega i j}}{\partial \omega_{i j}}=s_{i j} \xi_{i j}
$$


sendo

$$
\xi_{i j}=-\frac{1}{2 \mu_{i j}^{3}\left(1-\mu_{i j}\right)^{3}}\left\{1+\frac{\left(\mathrm{y}_{\omega i j}-\mu_{i j}\right)^{2}}{\mathrm{y}_{\omega i j}\left(1-\mathrm{y}_{\omega i j}\right)}\left[1+\frac{\mathrm{y}_{\omega i j}-2 \mathrm{y}_{\omega i j} \mu_{i j}+\mu_{i j}}{\mathrm{y}_{\omega i j}\left(1-\mathrm{y}_{\omega i j}\right)}\right]\right\}
$$

e $s_{i j}$ a raiz quadrada da variância definida em (1.9), com $i=1, \ldots, n$ e $j=1, \ldots, t$. Neste caso, matricialmente escrevemos

$$
\mathcal{B}=\mathcal{S} \boldsymbol{\Xi}
$$

sendo $\mathcal{S}=\operatorname{diag}\left(\mathcal{S}_{1}, \ldots, \mathcal{S}_{n}\right)$ e $\boldsymbol{\Xi}=\operatorname{diag}\left(\boldsymbol{\Xi}_{1}, \ldots, \boldsymbol{\Xi}_{n}\right)$, em que $\mathcal{S}_{i}=\operatorname{diag}\left(s_{i 1}, \ldots, s_{i t}\right)$ e $\boldsymbol{\Xi}_{i}=\operatorname{diag}\left(\xi_{i 1}, \ldots, \xi_{i t}\right), \operatorname{com} i=1, \ldots, n$.

\section{Modelos Lineares Generalizados}

Seguindo a equação de estimação definida em (4.3), temos que

$$
\mathrm{b}_{\omega i j}=\mathrm{y}_{\omega i j}-\mu_{i j}
$$

com $i=1, \ldots, n$ e $j=1, \ldots, t$. Considerando a definição de $\mathrm{y}_{\omega i j}$ em (6.10), a derivada de (6.17) com relação à perturbação $\omega_{i j}$ é dada por

$$
\frac{\partial \mathrm{b}_{\omega i j}}{\partial \omega_{i j}}=\sqrt{\operatorname{Var}\left(\mathrm{y}_{i j}\right)}=s_{i j}
$$

sendo $s_{i j}$ a raiz quadrada da variância definida em $(4.2), \operatorname{com} i=1, \ldots, n$ e $j=1, \ldots, t$. Assim, temos

$$
\mathcal{B}=\mathcal{S}
$$

sendo $\mathcal{S}=\operatorname{diag}\left(\mathcal{S}_{1}, \ldots, \mathcal{S}_{n}\right)$, em que $\mathcal{S}_{i}=\operatorname{diag}\left(s_{i 1}, \ldots, s_{i t}\right)$, com $i=1, \ldots, n$.

\subsubsection{Perturbação individual das covariáveis}

Considere um esquema aditivo de perturbação na $k$-ésima coluna da matriz de covariáveis $\mathbf{X}, \mathbf{x}_{k}=\left(\mathrm{x}_{11 k}, \mathrm{x}_{12 k}, \ldots, \mathrm{x}_{n t k}\right)^{\top}$, segundo proposta de Thomas e Cook (1990). Assim, o vetor perturbado $\mathbf{x}_{\omega k}$ tem cada componente dado por

$$
\mathrm{x}_{\omega i j k}=\mathrm{x}_{i j k}+\omega_{i j} s_{x_{k}}
$$

sendo $s_{x_{k}}$ um fator de escala dado pela desvio padrão de $\mathbf{x}_{k}, \operatorname{com} i=1, \ldots, n$ e $j=1, \ldots, t$. Assim, para exemplificar, se $k \neq 2$ e $k \neq p$, o modelo (2.2) perturbado segundo o esquema definido em (6.20) fica dado por

$$
g\left(\mu_{\omega i j}\right)=\eta_{\omega i j}=\beta_{1}+\mathrm{x}_{i j 2} \beta_{2}+\ldots+\mathrm{x}_{\omega i j k} \beta_{k}+\ldots+\mathrm{x}_{i j p} \beta_{p} .
$$


Neste tipo de perturbação, $\boldsymbol{\omega}_{0}=\mathbf{0}$.

Na equação de estimação definida em (6.6), todos os seus componentes são modificados quando perturbamos uma covariável. Logo, obtemos a seguinte equação de estimação perturbada

$$
\boldsymbol{\Psi}_{1}(\boldsymbol{\beta} \mid \boldsymbol{\omega})=\mathbf{X}_{\omega}^{\top} \Lambda_{\omega} \boldsymbol{\Omega}_{\omega}^{-1} \mathbf{b}_{\omega}
$$

em que o índice $\omega$ indica que as matrizes $\mathbf{X}, \boldsymbol{\Lambda}$ e $\boldsymbol{\Omega}$ e o vetor $\mathbf{b}$ dependem, de alguma forma, da perturbação definida em (6.20).

Por conseqüência, a derivada de $\boldsymbol{\Psi}_{1}(\boldsymbol{\beta} \mid \boldsymbol{\omega})$ com relação à $\boldsymbol{\omega}^{\top}$ pode ser expressa, segundo Harville (1997), por

$$
\boldsymbol{\Delta}=\mathbf{X}_{\omega}^{\top} \boldsymbol{\Lambda}_{\omega}\left[\boldsymbol{\Omega}_{\omega}^{-1} \frac{\partial \mathbf{b}_{\omega}}{\partial \boldsymbol{\omega}^{\top}}+\frac{\partial \boldsymbol{\Omega}_{\omega}^{-1}}{\partial \boldsymbol{\omega}^{\top}} \operatorname{diag}\left(\mathbf{b}_{\omega}\right)\right]+\left[\mathbf{X}_{\omega}^{\top} \frac{\partial \boldsymbol{\Lambda}_{\omega}}{\partial \boldsymbol{\omega}^{\top}}+\frac{\partial \mathbf{X}_{\omega}^{\top}}{\partial \boldsymbol{\omega}^{\top}} \boldsymbol{\Lambda}_{\omega}\right] \boldsymbol{\Omega}_{\omega}^{-1} \operatorname{diag}\left(\mathbf{b}_{\omega}\right)
$$

em que a derivada de $\mathbf{X}_{\omega}^{\top}$ com relação à $\boldsymbol{\omega}^{\top}$ é uma matriz $p \times N$ de zeros exceto a $k$-ésima linha que é composta pela constante $s_{x_{k}}, \operatorname{com} N=n t$, e

$$
\frac{\partial \boldsymbol{\Omega}_{\omega}^{-1}}{\partial \boldsymbol{\omega}^{\top}}=-\boldsymbol{\Omega}_{\omega}^{-1} \frac{\partial \boldsymbol{\Omega}_{\omega}}{\partial \boldsymbol{\omega}^{\top}} \boldsymbol{\Omega}_{\omega}^{-1}
$$

Entretanto, as derivadas de $\boldsymbol{\Lambda}_{\omega}, \boldsymbol{\Omega}_{\omega}$ e $\mathbf{b}_{\omega}$ todas com relação à $\boldsymbol{\omega}^{\top}$ são calculadas de acordo com a equação $\Psi_{1}(\boldsymbol{\beta})$ que pode ser dada, neste trabalho, pela equação de estimação (2.4), (3.3) ou (4.3).

Essas três equações de estimação representam, respectivamente, as equações de estimação generalizadas para modelos de regressão beta, modelos de regressão simplex e modelos lineares generalizados, todas sob homogeneidade da dispersão. As derivadas representadas por

$$
\begin{aligned}
& \frac{\partial \boldsymbol{\Lambda}_{\omega}}{\partial \boldsymbol{\omega}^{\top}}=\operatorname{diag}\left(\frac{\partial \boldsymbol{\Lambda}_{\omega 1}}{\partial \boldsymbol{\omega}_{1}^{\top}}, \ldots, \frac{\partial \boldsymbol{\Lambda}_{\omega n}}{\partial \boldsymbol{\omega}_{n}^{\top}}\right), \\
& \frac{\partial \boldsymbol{\Omega}_{\omega}}{\partial \boldsymbol{\omega}^{\top}}=\operatorname{diag}\left(\frac{\partial \boldsymbol{\Omega}_{\omega 1}}{\partial \boldsymbol{\omega}_{1}^{\top}}, \ldots, \frac{\partial \boldsymbol{\Omega}_{\omega n}}{\partial \boldsymbol{\omega}_{n}^{\top}}\right), \quad \mathrm{e} \\
& \frac{\partial \mathbf{b}_{\omega}}{\partial \boldsymbol{\omega}^{\top}}=\operatorname{diag}\left(\frac{\partial \mathbf{b}_{\omega 1}}{\partial \boldsymbol{\omega}_{1}^{\top}}, \ldots, \frac{\partial \mathbf{b}_{\omega n}}{\partial \boldsymbol{\omega}_{n}^{\top}}\right)
\end{aligned}
$$

são apresentadas, a seguir, para cada um desses três modelos.

\section{BETA}

De acordo com a equação de estimação definida em (2.4), temos

$$
\boldsymbol{\Lambda}_{\omega i}=\phi \mathbf{G}_{\omega i} \mathbf{A}_{\omega i}, \quad \boldsymbol{\Omega}_{\omega i}=\mathbf{A}_{\omega i}^{1 / 2} \mathbf{R}(\boldsymbol{\alpha}) \mathbf{A}_{\omega i}^{1 / 2} \quad \text { e } \quad \mathbf{b}_{\omega i}=\mathbf{y}_{i}^{*}-\boldsymbol{\mu}_{\omega i}^{*}
$$


sendo $\mathbf{G}_{\omega i}=\operatorname{diag}\left(\partial g^{-1}\left(\eta_{\omega i 1}\right) / \partial \eta_{\omega i 1}, \ldots, \partial g^{-1}\left(\eta_{\omega i t}\right) / \partial \eta_{\omega i t}\right), \mathbf{A}_{\omega i}=\operatorname{diag}\left(\mathrm{a}_{\omega i 1}, \ldots, \mathrm{a}_{\omega i t}\right)$ e $\boldsymbol{\mu}_{\omega i}^{*}=\left(\mu_{\omega i 1}^{*}, \ldots, \mu_{\omega i t}^{*}\right)^{\top}$, em que $\mathrm{a}_{\omega i j}=\psi^{\prime}\left(\mu_{\omega i j} \phi\right)+\psi^{\prime}\left(\left(1-\mu_{\omega i j}\right) \phi\right)$ e $\mu_{\omega i j}^{*}=\psi\left(\mu_{\omega i j} \phi\right)-$ $\psi\left(\left(1-\mu_{\omega i j}\right) \phi\right)$, com $i=1, \ldots, n$ e $j=1, \ldots, t$. Assim, da Seção B.1.2 do Apêndice B, obtemos

$$
\begin{aligned}
& \frac{\partial \boldsymbol{\Lambda}_{\omega i}}{\partial \boldsymbol{\omega}_{i}^{\top}}=\phi \beta_{k} s_{x_{k}}\left[\dot{\mathbf{G}}_{\omega i} \mathbf{A}_{\omega i}+\mathbf{G}_{\omega i}^{2} \mathcal{E}_{1 \omega i}\right] \\
& \frac{\partial \boldsymbol{\Omega}_{\omega i}}{\partial \boldsymbol{\omega}_{i}^{\top}}=\frac{1}{2} \beta_{k} s_{x_{k}}\left[\mathbf{A}_{\omega i}^{1 / 2} \mathbf{R}(\boldsymbol{\alpha}) \mathcal{A}_{\omega i}+\mathcal{A}_{\omega i} \mathbf{R}(\boldsymbol{\alpha}) \mathbf{A}_{\omega i}^{1 / 2}\right], \quad \mathrm{e} \\
& \frac{\partial \mathbf{b}_{\omega i}}{\partial \boldsymbol{\omega}_{i}^{\top}}=-\phi \beta_{k} s_{x_{k}} \mathbf{G}_{\omega i} \mathbf{A}_{\omega i}
\end{aligned}
$$

sendo $\dot{\mathbf{G}}_{\omega i}=\operatorname{diag}\left(\partial^{2} g^{-1}\left(\eta_{\omega i 1}\right) / \partial \eta_{\omega i 1}^{2}, \ldots, \partial^{2} g^{-1}\left(\eta_{\omega i t}\right) / \partial \eta_{\omega i t}^{2}\right), \quad \mathcal{A}_{\omega i}=\mathbf{A}_{\omega i}^{-1 / 2} \mathbf{G}_{\omega i} \mathcal{E}_{1 \omega i}$ e $\mathcal{E}_{1 \omega i}=\operatorname{diag}\left(\mathcal{E}_{1 \omega i 1}, \ldots, \mathcal{E}_{1 \omega i t}\right)$, em que

$$
\mathcal{E}_{1 \omega i j}=\phi\left[\psi^{\prime \prime}\left(\mu_{\omega i j} \phi\right)-\psi^{\prime \prime}\left(\left(1-\mu_{\omega i j}\right) \phi\right)\right]
$$

com a função poligama $\psi^{\prime \prime}(\cdot)$ avaliada no ponto $\lambda$ dada por

$$
\psi^{\prime \prime}(\lambda)=\frac{\mathrm{d} \psi^{\prime}(\lambda)}{\mathrm{d} \lambda}=\frac{\mathrm{d}^{3} \log \Gamma(\lambda)}{\mathrm{d} \lambda^{3}}=\frac{\Gamma^{\prime \prime \prime}(\lambda) \Gamma(\lambda)-\Gamma^{\prime \prime}(\lambda) \Gamma^{\prime}(\lambda)}{\Gamma(\lambda)^{2}}-\psi(\lambda)^{2},
$$

$i=1, \ldots, n$ e $j=1, \ldots, t$.

No caso em que todas as observações são independentes entre si, nossa matriz $\boldsymbol{\Delta}$ definida para perturbação individual de covariáveis coincide com a mesma matriz apresentada por Ospina (2007, Seção 3.4.3).

\section{Simplex}

De acordo com a equação de estimação definida em (3.3), temos

$$
\boldsymbol{\Lambda}_{\omega i}=\phi \mathbf{G}_{\omega i} \mathbf{A}_{\omega i}, \quad \boldsymbol{\Omega}_{\omega i}=\mathbf{A}_{\omega i}^{1 / 2} \mathbf{R}(\boldsymbol{\alpha}) \mathbf{A}_{\omega i}^{1 / 2} \quad \text { e } \quad \mathbf{b}_{\omega i}=\mathbf{u}_{\omega i}
$$

sendo $\mathbf{G}_{\omega i}=\operatorname{diag}\left(\partial g^{-1}\left(\eta_{\omega i 1}\right) / \partial \eta_{\omega i 1}, \ldots, \partial g^{-1}\left(\eta_{\omega i t}\right) / \partial \eta_{\omega i t}\right), \mathbf{A}_{\omega i}=\operatorname{diag}\left(\mathbf{a}_{\omega i 1}, \ldots, \mathrm{a}_{\omega i t}\right)$ e $\mathbf{u}_{\omega i}=\left(\mathrm{u}_{\omega i 1}, \ldots, \mathrm{u}_{\omega i t}\right)^{\top}$, em que

$$
\mathrm{a}_{\omega i j}=\phi^{-1}\left[\frac{3 \phi^{-1}}{\mu_{\omega i j}\left(1-\mu_{\omega i j}\right)}+\frac{1}{\mu_{\omega i j}^{3}\left(1-\mu_{\omega i j}\right)^{3}}\right]
$$

$\mathrm{e}$

$$
\mathrm{u}_{\omega i j}=-\frac{1}{2} \frac{\partial d\left(\mathrm{y}_{i j} ; \mu_{\omega i j}\right)}{\partial \mu_{\omega i j}}=\frac{\mathrm{y}_{i j}-\mu_{\omega i j}}{\mu_{\omega i j}\left(1-\mu_{\omega i j}\right)}\left[d\left(\mathrm{y}_{i j} ; \mu_{\omega i j}\right)+\frac{1}{\mu_{\omega i j}^{2}\left(1-\mu_{\omega i j}\right)^{2}}\right]
$$


com $i=1, \ldots, n$ e $j=1, \ldots, t$. Assim, da Seção B.1.2 do Apêndice B, obtemos

$$
\begin{aligned}
& \frac{\partial \boldsymbol{\Lambda}_{\omega i}}{\partial \boldsymbol{\omega}_{i}^{\top}}=\phi \beta_{k} s_{x_{k}}\left[\dot{\mathbf{G}}_{\omega i} \mathbf{A}_{\omega i}+\mathbf{G}_{\omega i}^{2} \mathcal{E}_{1 \omega i}\right], \\
& \frac{\partial \boldsymbol{\Omega}_{\omega i}}{\partial \boldsymbol{\omega}_{i}^{\top}}=\frac{1}{2} \phi \beta_{k} s_{x_{k}}\left[\mathbf{A}_{\omega i}^{1 / 2} \mathbf{R}(\boldsymbol{\alpha}) \mathcal{A}_{\omega i}+\mathcal{A}_{\omega i} \mathbf{R}(\boldsymbol{\alpha}) \mathbf{A}_{\omega i}^{1 / 2}\right] \quad \mathrm{e} \\
& \frac{\partial \mathbf{b}_{\omega i}}{\partial \boldsymbol{\omega}_{i}^{\top}}=\beta_{k} s_{x_{k}} \mathbf{G}_{\omega i} \mathcal{U}_{\omega i},
\end{aligned}
$$

sendo $\dot{\mathbf{G}}_{\omega i}=\operatorname{diag}\left(\partial^{2} g^{-1}\left(\eta_{\omega i 1}\right) / \partial \eta_{\omega i 1}^{2}, \ldots, \partial^{2} g^{-1}\left(\eta_{\omega i t}\right) / \partial \eta_{\omega i t}^{2}\right), \quad \mathcal{A}_{\omega i}=\mathbf{A}_{\omega i}^{-1 / 2} \mathbf{G}_{\omega i} \mathcal{E}_{1 \omega i}$, $\mathcal{E}_{1 \omega i}=\operatorname{diag}\left(\mathcal{E}_{1 \omega i 1}, \ldots, \mathcal{E}_{1 \omega i t}\right)$ e $\mathcal{U}_{\omega i}=\operatorname{diag}\left(\mathcal{U}_{\omega i 1}, \ldots, \mathcal{U}_{\omega i t}\right)$, em que

$$
\mathcal{E}_{1 \omega i j}=-3 \phi^{-1}\left[\frac{\phi^{-1}\left(1-2 \mu_{\omega i j}\right)}{\left(\mu_{\omega i j}\left(1-\mu_{\omega i j}\right)\right)^{2}}+\frac{\mu_{\omega i j}^{2}\left(1-\mu_{\omega i j}\right)^{3}-\mu_{\omega i j}^{3}\left(1-\mu_{\omega i j}\right)^{2}}{\left(\mu_{\omega i j}^{3}\left(1-\mu_{\omega i j}\right)^{3}\right)^{2}}\right]
$$

$\mathrm{e}$

$$
\begin{aligned}
\mathcal{U}_{\omega i j}=\quad & -\frac{\mu_{\omega i j}\left(1-\mu_{\omega i j}\right)+2 \mu_{\omega i j}\left(\mathrm{y}_{\omega i j}-\mu_{\omega i j}\right)}{\left(\mu_{\omega i j}\left(1-\mu_{\omega i j}\right)\right)^{2}}\left[d\left(\mathrm{y}_{i j} ; \mu_{\omega i j}\right)+\frac{1}{\mu_{\omega i j}^{2}\left(1-\mu_{\omega i j}\right)^{2}}\right]- \\
& -\frac{\left(\mathrm{y}_{\omega i j}-\mu_{\omega i j}\right)}{\mu_{\omega i j}\left(1-\mu_{\omega i j}\right)}\left[2 \mathrm{u}_{\omega i j}+\frac{2 \mu_{\omega i j}-6 \mu_{\omega i j}^{2}+4 \mu_{\omega i j}^{3}}{\left(\mu_{\omega i j}^{2}\left(1-\mu_{\omega i j}\right)^{2}\right)^{2}}\right]
\end{aligned}
$$

com $i=1, \ldots, n$ e $j=1, \ldots, t$.

\section{Modelos Lineares Generalizados}

De acordo com a equação de estimação definida em (4.3), temos

$$
\boldsymbol{\Lambda}_{\omega i}=\mathbf{G}_{\omega i}, \quad \boldsymbol{\Omega}_{\omega i}=\phi \mathbf{A}_{\omega i}^{1 / 2} \mathbf{R}(\boldsymbol{\alpha}) \mathbf{A}_{\omega i}^{1 / 2} \quad \text { e } \quad \mathbf{b}_{\omega i}=\mathbf{y}_{i}-\boldsymbol{\mu}_{\omega i}
$$

sendo $\mathbf{G}_{\omega i}=\operatorname{diag}\left(\partial g^{-1}\left(\eta_{\omega i 1}\right) / \partial \eta_{\omega i 1}, \ldots, \partial g^{-1}\left(\eta_{\omega i t}\right) / \partial \eta_{\omega i t}\right), \mathbf{A}_{\omega i}=\operatorname{diag}\left(\mathrm{a}_{\omega i 1}, \ldots, \mathrm{a}_{\omega i t}\right)$ e $\boldsymbol{\mu}_{\omega i}=\left(\mu_{\omega i 1}, \ldots, \mu_{\omega i t}\right)^{\top}$, em que $\mathrm{a}_{\omega i j}=v\left(\mu_{\omega i j}\right)$, com $i=1, \ldots, n$ e $j=1, \ldots, t$. Assim, obtemos

$$
\begin{aligned}
& \frac{\partial \boldsymbol{\Lambda}_{\omega i}}{\partial \boldsymbol{\omega}_{i}^{\top}}=\beta_{k} s_{x_{k}} \dot{\mathbf{G}}_{\omega i}, \\
& \frac{\partial \boldsymbol{\Omega}_{\omega i}}{\partial \boldsymbol{\omega}_{i}^{\top}}=\frac{1}{2} \phi \beta_{k} s_{x_{k}}\left[\mathbf{A}_{\omega i}^{1 / 2} \mathbf{R}(\boldsymbol{\alpha}) \mathcal{A}_{\omega i}+\mathcal{A}_{\omega i} \mathbf{R}(\boldsymbol{\alpha}) \mathbf{A}_{\omega i}^{1 / 2}\right], \quad \mathrm{e} \\
& \frac{\partial \mathbf{b}_{\omega i}}{\partial \boldsymbol{\omega}_{i}^{\top}}=-\beta_{k} s_{x_{k}} \mathbf{G}_{\omega i},
\end{aligned}
$$

sendo $\dot{\mathbf{G}}_{\omega i}=\operatorname{diag}\left(\partial^{2} g^{-1}\left(\eta_{\omega i 1}\right) / \partial \eta_{\omega i 1}^{2}, \ldots, \partial^{2} g^{-1}\left(\eta_{\omega i t}\right) / \partial \eta_{\omega i t}^{2}\right)$ e $\mathcal{A}_{\omega i}=\mathbf{A}_{\omega i}^{-1 / 2} \mathbf{G}_{\omega i} \dot{\boldsymbol{\Upsilon}}_{\omega i}$, em que $\dot{\Upsilon}_{\omega i}=\operatorname{diag}\left(\partial v\left(\mu_{\omega i 1}\right) / \partial \mu_{\omega i 1}, \ldots, \partial v\left(\mu_{\omega i t}\right) / \partial \mu_{\omega i t}\right)$, com $i=1, \ldots, n$ e $j=1, \ldots, t$. A Tabela C.4 do Apêndice $\mathrm{C}$ apresenta a derivada de $v\left(\mu_{\omega}\right)$ com relação à $\mu_{\omega}$ para algumas distribuições pertencentes à família exponencial. 


\subsubsection{Perturbação do parâmetro de precisão}

Considere um esquema de perturbação do parâmetro de precisão, de forma que esse não seja constante ao longo das observações e nem entre os indivíduos, ou seja,

$$
\phi_{\omega i j}=\frac{\phi}{\omega_{i j}}
$$

com $i=1, \ldots, n$ e $j=1, \ldots, t$. Neste tipo de perturbação, $\boldsymbol{\omega}_{0}=1$. Da equação (6.6), obtemos a seguinte equação de estimação perturbada

$$
\boldsymbol{\Psi}_{1}(\boldsymbol{\beta} \mid \boldsymbol{\omega})=\mathbf{X}^{\top} \boldsymbol{\Lambda}_{\omega} \boldsymbol{\Omega}_{\omega}^{-1} \mathbf{b}_{\omega}
$$

em que o índice $\omega$ indica que as matrizes $\Lambda$ e $\Omega$ e o vetor $\mathbf{b}$ dependem, de alguma forma, da perturbação definida em (6.30). Assim, segundo Harville (1997), a matriz definida em (6.2) pode ser expressa por

$$
\boldsymbol{\Delta}=\mathbf{X}^{\top} \boldsymbol{\Lambda}_{\omega} \boldsymbol{\Omega}_{\omega}^{-1} \frac{\partial \mathbf{b}_{\omega}}{\partial \boldsymbol{\omega}^{\top}}+\mathbf{X}^{\top} \boldsymbol{\Lambda}_{\omega} \frac{\partial \boldsymbol{\Omega}_{\omega}^{-1}}{\partial \boldsymbol{\omega}^{\top}} \operatorname{diag}\left(\mathbf{b}_{\omega}\right)+\mathbf{X}^{\top} \frac{\partial \boldsymbol{\Lambda}_{\omega}}{\partial \boldsymbol{\omega}^{\top}} \boldsymbol{\Omega}_{\omega}^{-1} \operatorname{diag}\left(\mathbf{b}_{\omega}\right),
$$

em que as derivadas de $\boldsymbol{\Lambda}_{\omega}, \boldsymbol{\Omega}_{\omega}^{-1}$ e $\mathbf{b}_{\omega}$ todas com relação à $\boldsymbol{\omega}^{\top}$ são dadas, respectivamente, em (6.22), (6.21) e (6.23).

Neste trabalho, a equação $\boldsymbol{\Psi}_{1}(\boldsymbol{\beta} \mid \boldsymbol{\omega})$ que pode ser definida a partir da equações de estimação (2.4), (3.3) e (4.3). A seguir, apresentamos como a medida de influência local com perturbação do parâmetro de precisão fica descrita para cada um desses três casos.

BETA

De acordo com a equação de estimação definida em (2.4), temos

$$
\boldsymbol{\Lambda}_{\omega i}=\mathbf{G}_{i} \boldsymbol{\Phi}_{\omega i} \mathbf{A}_{\omega i}, \quad \boldsymbol{\Omega}_{\omega i}=\mathbf{A}_{\omega i}^{1 / 2} \mathbf{R}(\boldsymbol{\alpha}) \mathbf{A}_{\omega i}^{1 / 2} \quad \text { e } \quad \mathbf{b}_{\omega i}=\mathbf{y}_{i}^{*}-\boldsymbol{\mu}_{\omega i}^{*}
$$

sendo $\boldsymbol{\Phi}_{\omega i}=\operatorname{diag}\left(\phi_{\omega i 1}, \ldots, \phi_{\omega i t}\right), \mathbf{A}_{\omega i}=\operatorname{diag}\left(\mathrm{a}_{\omega i 1}, \ldots, \mathrm{a}_{\omega i t}\right)$ e $\boldsymbol{\mu}_{\omega i}^{*}=\left(\mu_{\omega i 1}^{*}, \ldots, \mu_{\omega i t}^{*}\right)^{\top}$, em que $\mathrm{a}_{\omega i j}=\psi^{\prime}\left(\mu_{i j} \phi_{\omega i j}\right)+\psi^{\prime}\left(\left(1-\mu_{i j}\right) \phi_{\omega i j}\right)$ e $\mu_{\omega i j}^{*}=\psi\left(\mu_{i j} \phi_{\omega i j}\right)-\psi\left(\left(1-\mu_{i j}\right) \phi_{\omega i j}\right)$, com $i=1, \ldots, n$ e $j=1, \ldots, t$. Assim, da Seção B.1.2 do Apêndice B, obtemos

$$
\begin{aligned}
& \frac{\partial \boldsymbol{\Lambda}_{\omega i}}{\partial \boldsymbol{\omega}_{i}^{\top}}=-\phi^{-1} \mathbf{G}_{i} \boldsymbol{\Phi}_{\omega i}^{2}\left[\boldsymbol{\Phi}_{\omega i} \mathcal{E}_{2 \omega i}+\mathbf{A}_{\omega i}\right], \\
& \frac{\partial \boldsymbol{\Omega}_{\omega i}}{\partial \boldsymbol{\omega}_{i}^{\top}}=-\frac{1}{2} \phi^{-1}\left[\mathbf{A}_{\omega i}^{1 / 2} \mathbf{R}(\boldsymbol{\alpha}) \mathcal{A}_{\omega i}+\mathcal{A}_{\omega i} \mathbf{R}(\boldsymbol{\alpha}) \mathbf{A}_{\omega i}^{1 / 2}\right], \quad \mathrm{e} \\
& \frac{\partial \mathbf{b}_{\omega i}}{\partial \boldsymbol{\omega}_{i}^{\top}}=\phi^{-1} \boldsymbol{\Phi}_{\omega i}^{2} \mathcal{E}_{3 \omega i},
\end{aligned}
$$


sendo $\mathcal{A}_{\omega i}=\mathbf{A}_{\omega i}^{-1 / 2} \boldsymbol{\Phi}_{\omega i}^{2} \mathcal{E}_{2 \omega i}, \quad \mathcal{E}_{2 \omega i}=\operatorname{diag}\left(\mathcal{E}_{2 \omega i 1}, \ldots, \mathcal{E}_{2 \omega i t}\right)$ e $\mathcal{E}_{3 \omega i}=\operatorname{diag}\left(\mathcal{E}_{3 \omega i 1}, \ldots, \mathcal{E}_{3 \omega i t}\right)$, em que

$$
\mathcal{E}_{2 \omega i j}=\left[\mu_{i j} \psi^{\prime \prime}\left(\mu_{i j} \phi_{\omega i j}\right)+\left(1-\mu_{i j}\right) \psi^{\prime \prime}\left(\left(1-\mu_{i j}\right) \phi_{\omega i j}\right)\right]
$$

e

$$
\mathcal{E}_{3 \omega i}=\left[\mu_{i j} \psi^{\prime}\left(\mu_{i j} \phi_{\omega i j}\right)-\left(1-\mu_{i j}\right) \psi^{\prime}\left(\left(1-\mu_{i j}\right) \phi_{\omega i j}\right)\right]
$$

com $i=1, \ldots, n$ e $j=1, \ldots, t$.

No caso em que todas as observações são independentes entre si, nossa medida de influência local para perturbação individual de covariáveis coincide com a medida de influência apresentada por Ospina (2007, Seção 3.4.4)

\section{SimpleX}

De acordo com a equação de estimação definida em (3.3), temos

$$
\boldsymbol{\Lambda}_{\omega i}=\mathbf{G}_{i} \boldsymbol{\Phi}_{\omega i} \mathbf{A}_{\omega i} \quad \text { e } \quad \boldsymbol{\Omega}_{\omega i}=\mathbf{A}_{\omega i}^{1 / 2} \mathbf{R}(\boldsymbol{\alpha}) \mathbf{A}_{\omega i}^{1 / 2}
$$

sendo $\boldsymbol{\Phi}_{\omega i}=\operatorname{diag}\left(\phi_{\omega i 1}, \ldots, \phi_{\omega i t}\right)$ e $\mathbf{A}_{\omega i}=\operatorname{diag}\left(\mathrm{a}_{\omega i 1}, \ldots, \mathrm{a}_{\omega i t}\right)$, em que $\mathrm{a}_{\omega i j}=\phi_{\omega i j}^{-1}$ $\left[3 \phi_{\omega i j}^{-1} /\left(\mu_{i j}\left(1-\mu_{i j}\right)\right)+1 /\left(\mu_{i j}^{3}\left(1-\mu_{i j}\right)^{3}\right)\right]$, com $i=1, \ldots, n$ e $j=1, \ldots, t$. Notemos que para a equação (3.3), o vetor $\mathbf{b}_{1 i}$ não depende de $\phi$, fazendo com que a primeira parcela de (6.31) valha zero. Assim, da Seção B.1.2 do Apêndice B, obtemos

$$
\begin{aligned}
& \frac{\partial \boldsymbol{\Lambda}_{\omega i}}{\partial \boldsymbol{\omega}_{i}^{\top}}=-\phi^{-1} \mathbf{G}_{i} \boldsymbol{\Phi}_{\omega i}^{2}\left[\boldsymbol{\Phi}_{\omega i} \mathcal{E}_{2 \omega i}+\mathbf{A}_{\omega i}\right], \quad \mathrm{e} \\
& \frac{\partial \boldsymbol{\Omega}_{\omega i}}{\partial \boldsymbol{\omega}_{i}^{\top}}=-\frac{1}{2} \phi^{-1}\left[\mathbf{A}_{\omega i}^{1 / 2} \mathbf{R}(\boldsymbol{\alpha}) \mathcal{A}_{\omega i}+\mathcal{A}_{\omega i} \mathbf{R}(\boldsymbol{\alpha}) \mathbf{A}_{\omega i}^{1 / 2}\right]
\end{aligned}
$$

sendo $\mathcal{A}_{\omega i}=\mathbf{A}_{\omega i}^{-1 / 2} \boldsymbol{\Phi}_{\omega i}^{2} \mathcal{E}_{2 \omega i}$ e $\mathcal{E}_{2 \omega i}=\operatorname{diag}\left(\mathcal{E}_{2 \omega i 1}, \ldots, \mathcal{E}_{2 \omega i t}\right)$, em que

$$
\mathcal{E}_{2 \omega i j}=-\phi_{\omega i j}^{-2}\left[\frac{6 \phi_{\omega i j}^{-1}}{\mu_{i j}\left(1-\mu_{i j}\right)}+\frac{1}{\mu_{i j}^{3}\left(1-\mu_{i j}\right)^{3}}\right] \text {, }
$$

$\operatorname{com} i=1, \ldots, n$ e $j=1, \ldots, t$.

\section{Modelos Lineares Generalizados}

De acordo com a equação de estimação definida em (4.3), temos

$$
\Omega_{\omega i}=\Phi_{\omega i} \mathbf{A}_{i}^{1 / 2} \mathbf{R}(\boldsymbol{\alpha}) \mathbf{A}_{i}^{1 / 2}
$$


sendo $\boldsymbol{\Phi}_{\omega i}=\operatorname{diag}\left(\phi_{\omega i 1}, \ldots, \phi_{\omega i t}\right)$, com $i=1, \ldots, n$ e $j=1, \ldots, t$. Notemos que para a equação (4.3), a matriz $\boldsymbol{\Lambda}_{i}$ e o vetor $\mathbf{b}_{1 i}$ não dependem de $\phi$, fazendo com que a primeira e terceira parcelas de (6.31) valham zero. Assim, temos

$$
\frac{\partial \boldsymbol{\Lambda}_{\omega i}}{\partial \boldsymbol{\omega}_{i}^{\top}}=\frac{\partial \boldsymbol{\Phi}_{\omega i}}{\partial \boldsymbol{\omega}_{i}^{\top}} \mathbf{A}_{i}^{1 / 2} \mathbf{R}(\boldsymbol{\alpha}) \mathbf{A}_{i}^{1 / 2}=-\phi^{-1} \boldsymbol{\Phi}_{\omega i}^{2} \mathbf{A}_{i}^{1 / 2} \mathbf{R}(\boldsymbol{\alpha}) \mathbf{A}_{i}^{1 / 2}
$$

com $i=1, \ldots, n$ e $j=1, \ldots, t$.

\subsubsection{Perturbação na matriz de correlação de trabalho $\mathbf{R}(\alpha)$}

Inicialmente, seja $\mathbf{R}(\boldsymbol{\alpha})$ uma matriz de correlação de trabalho definida numa forma geral dada pelo vetor de correlações $\boldsymbol{\alpha}=\left(\alpha_{11}, \alpha_{12}, \ldots, \alpha_{(t-1) t}, \alpha_{t t}\right)^{\top}$, em que $\alpha_{j j}=1 \mathrm{e}$ $\alpha_{j j^{\prime}}=\alpha_{j^{\prime} j}$, com $j \neq j^{\prime}$ e $j, j^{\prime}=1, \ldots, t$. Para exemplificar, se a matriz de correlação de trabalho for a padrão uniforme, então $\alpha_{j j^{\prime}}=\alpha$; se for a auto-regressiva de ordem 1 (AR-1), então $\alpha_{j j^{\prime}}=\alpha^{\left|j-j^{\prime}\right|}$, para qualquer $j \neq j^{\prime}$ e $j, j^{\prime}=1, \ldots, t$. Lembramos que a matriz de correlação de trabalho proposta para modelar os dados é sempre a mesma para todas as unidades experimentais $i, i=1, \ldots, n$ (ver Parte I).

Um possível esquema de perturbação no vetor de correlações $\boldsymbol{\alpha}$ de forma que esse não seja o mesmo entre as unidades experimentais e nem ao longo das observações pode ser dado por

$$
\alpha_{\omega i\left(j j^{\prime}\right)}=\frac{\alpha_{j j^{\prime}}}{\omega_{i\left(j j^{\prime}\right)}}
$$

sendo $\alpha_{j j^{\prime}}=1$ quando $j=j^{\prime}$ e $\alpha_{j j^{\prime}}=\alpha_{j^{\prime} j}$ quando $j \neq j^{\prime}, \operatorname{com} i=1, \ldots, n$ e $j, j^{\prime}=1, \ldots, t$. Nesse tipo de perturbação, $\boldsymbol{\omega}_{0}=\mathbf{1}$.

A equação de estimação (6.6) perturbada segundo o esquema (6.38) é dada por

$$
\Psi_{1}(\boldsymbol{\beta} \mid \boldsymbol{\omega})=\mathbf{X}^{\top} \Lambda \Omega_{\omega}^{-1} \mathbf{b}
$$

alterando apenas a matriz $\Omega$ com essa perturbação. Assim, a matriz definida em (6.2) pode ser expressa por

$$
\boldsymbol{\Delta}=\mathbf{X}^{\top} \boldsymbol{\Lambda} \frac{\partial \boldsymbol{\Omega}_{\omega}^{-1}}{\partial \boldsymbol{\omega}^{\top}} \operatorname{diag}(\mathbf{b})
$$

sendo a derivada de $\boldsymbol{\Omega}_{\omega}^{-1}$ com relação à $\boldsymbol{\omega}^{\top}$ dada em (6.21) e

$$
\frac{\partial \boldsymbol{\Omega}_{\omega}}{\partial \boldsymbol{\omega}^{\top}}=\operatorname{Var}(\mathbf{b})^{1 / 2} \frac{\partial \mathbf{R}\left(\boldsymbol{\alpha}_{\omega}\right)}{\partial \boldsymbol{\omega}^{\top}} \operatorname{Var}(\mathbf{b})^{1 / 2}
$$

em que $\partial \alpha_{\omega i\left(j j^{\prime}\right)} / \partial \omega_{i\left(j j^{\prime}\right)}=-\alpha_{j j^{\prime}} / \omega_{i\left(j j^{\prime}\right)}^{2}$, qualquer que seja a estrutura da matriz de cor- 
relação de trabalho. Dessa forma, a derivada de $\Omega_{\omega}$ com relação à $\boldsymbol{\omega}^{\top}$ avaliada no vetor com ausência de perturbação, aqui $\boldsymbol{\omega}_{0}=\mathbf{1}$, é dada por $-\Omega$.

Com base na equação (6.5), a medida de influência local com perturbação na matriz de correlação de trabalho é obtida da matriz

$$
\operatorname{diag}(\mathbf{b}) \boldsymbol{\Omega}^{-1} \boldsymbol{\Lambda}^{\top} \mathbf{X}\left(\mathbf{X}^{\top} \mathbf{W} \mathbf{X}\right)^{-1} \mathbf{X}^{\top} \boldsymbol{\Lambda} \boldsymbol{\Omega}^{-1} \operatorname{diag}(\mathbf{b})
$$

avaliada em $\boldsymbol{\omega}_{0}$ e em $\left(\hat{\boldsymbol{\beta}}^{\top}, \hat{\phi}\right)^{\top}$.

A matriz definida na equação (6.39) é a mesma descrita na equação (6.9) da qual obtemos a medida de influência local para ponderação de casos. Isso indica que a ponderação de casos, além de ser interpretada como uma perturbação na variância de cada observação, também pode ser vista como uma perturbação na matriz de correlação de trabalho.

\subsection{Esquemas de perturbação sob heterogeneidade da dispersão}

As equações de estimação generalizadas para modelagem conjunta da média e da dispersão estão definidas nas equações (2.13), (3.12) e (4.10) para os modelos de regressão beta, modelos de regressão simplex e modelos lineares generalizados, respectivamente. De forma geral, essas equações de estimação podem ser escritas na forma geral

$$
\Psi_{2}(\boldsymbol{\theta})=\mathbf{M}^{\top} \boldsymbol{\Lambda} \boldsymbol{\Omega}^{-1} \mathbf{b}=\mathbf{M}^{\top} \mathbf{W} \Lambda^{-\top} \mathbf{b}
$$

cuja matriz de sensibilidade, conforme definição em (1.12), é expressa por:

$$
\mathbf{S}=-\mathbf{M}^{\top} \mathbf{W M}
$$

em que $\mathbf{M}=\left(\mathbf{M}_{1}^{\top}, \ldots, \mathbf{M}_{n}^{\top}\right)^{\top}, \boldsymbol{\Lambda}=\operatorname{diag}\left(\boldsymbol{\Lambda}_{1}, \ldots, \boldsymbol{\Lambda}_{n}\right), \boldsymbol{\Omega}=\operatorname{diag}\left(\boldsymbol{\Omega}_{1}, \ldots, \boldsymbol{\Omega}_{n}\right), \mathbf{W}=$ $\operatorname{diag}\left(\mathbf{W}_{1}, \ldots, \mathbf{W}_{n}\right)$ e $\mathbf{b}=\left(\mathbf{b}_{1}^{\top}, \ldots, \mathbf{b}_{n}^{\top}\right)^{\top}$. Notemos que aqui a matriz $\boldsymbol{\Lambda}$ definida para modelos de regressão beta de acordo com a equação (2.13) não é uma matriz quadrada sendo importante distinguir sua matriz transposta quando conveniente.

As medidas de influência local, descritas a seguir para alguns esquemas de perturbação com heterogeneidade da dispersão, são definidas a partir das equações (6.40) e (6.41), avaliadas sob as estimativas do modelo postulado $\hat{\boldsymbol{\theta}}^{\top}$ e em $\boldsymbol{\omega}_{0}$. 


\subsubsection{Ponderação de casos}

Considere o esquema de perturbação

$$
\boldsymbol{\Psi}_{2}(\boldsymbol{\theta} \mid \boldsymbol{\omega})=\mathbf{M}^{\top} \mathbf{W} \boldsymbol{\Lambda}^{-\top} \operatorname{diag}(\boldsymbol{\omega}) \mathbf{b}
$$

em que $\boldsymbol{\omega}=\left(\boldsymbol{\omega}_{1}^{\top}, \ldots, \boldsymbol{\omega}_{n}^{\top}\right)^{\top}, \operatorname{com} \boldsymbol{\omega}_{i}=\left(\omega_{i 1}, \ldots, \omega_{i t}\right)^{\top}, i=1, \ldots, n$. Aqui, $\boldsymbol{\omega}_{0}$ que representa ausência de perturbação é um vetor de uns.

Assim, a matriz $\boldsymbol{\Delta}$ que equivale a derivada $\boldsymbol{\Psi}_{2}(\boldsymbol{\theta} \mid \boldsymbol{\omega})$, definida em (6.42), com relação à $\boldsymbol{\omega}^{\top}$ é dada por $\boldsymbol{\Delta}=\mathbf{M}^{\top} \mathbf{W} \boldsymbol{\Lambda}^{-\top} \mathbf{B}$, sendo

$$
\mathbf{B}=\operatorname{diag}(\mathbf{b}), \quad \text { para BETA }
$$

$\mathrm{Ou}$

$$
\mathbf{B}=\left(\begin{array}{c}
\operatorname{diag}\left(\mathbf{b}_{1}\right) \\
\operatorname{diag}\left(\mathbf{b}_{2}\right)
\end{array}\right), \quad \text { para Simplex e Modelos Lineares Generalizados. }
$$

Nesse caso, a matriz definida em (6.5) é dada por

$$
\mathbf{B} \Lambda^{-1} \mathbf{W}^{\top} \mathbf{M}\left(\mathbf{M}^{\top} \mathbf{W} \mathbf{M}\right)^{-1} \mathbf{M}^{\top} \mathbf{M} \Lambda^{-\top} \mathbf{B}
$$

a qual deve ser avaliada em $\hat{\boldsymbol{\theta}}$ e em $\boldsymbol{\omega}_{0}$.

\subsubsection{Perturbação da variável resposta}

Considerando $\mathbf{b}_{\omega}$ o vetor $\mathbf{b}$ com perturbação na variável resposta conforme o esquema expresso em (6.10), a equação de estimação perturbada fica escrita na forma

$$
\boldsymbol{\Psi}_{2}(\boldsymbol{\theta} \mid \boldsymbol{\omega})=\mathbf{M}^{\top} \mathbf{W} \boldsymbol{\Lambda}^{-\top} \mathbf{b}_{\omega},
$$

em que $\mathbf{b}_{\omega}=\left(\mathbf{b}_{\omega 1}^{\top}, \ldots, \mathbf{b}_{\omega n}^{\top}\right)^{\top}$, com $\mathbf{b}_{\omega i}=\left(\mathrm{b}_{\omega i 1}, \ldots, \mathrm{b}_{\omega i t}\right)^{\top}, i=1, \ldots, n$.

Logo, com base na equação (6.5), a medida de influência local com perturbação da variável resposta é obtida da matriz

$$
\mathcal{B}^{\top} \Lambda^{-1} \mathbf{W}^{\top} \mathbf{M}\left(\mathbf{M}^{\top} \mathbf{W} \mathbf{M}\right)^{-1} \mathbf{M}^{\top} \mathbf{W} \Lambda^{-\top} \mathcal{B}
$$

avaliada em $\hat{\boldsymbol{\theta}}$ e em $\boldsymbol{\omega}_{0}, \operatorname{com} \mathcal{B}=\partial \mathbf{b}_{\omega} / \partial \boldsymbol{\omega}^{\top}$.

A seguir, apresentamos a derivada do vetor perturbado $\mathbf{b}_{\omega}$ com relação ao vetor de perturbação $\boldsymbol{\omega}$ para as equações (2.13), (3.12) e (4.10), descritas, respectivamente, para os modelos de regressão beta, modelos de regressão simplex e modelos lineares generalizados. 
BETA

Para a equação de estimação definida em (2.13), o vetor perturbado na variável resposta referente à $i$-ésima unidade experimental $\mathbf{b}_{\omega i}$ é dado pela equação (6.11). Dessa forma, também para o caso de modelagem conjunta da média e da dispersão, $\mathcal{B}$ fica expresso conforme (6.12), mas com $s_{i j}=\sqrt{v\left(\mu_{i j}\right) /\left(1+\phi_{i j}\right)}, i=1, \ldots, n$ e $j=1, \ldots, t$.

\section{SimpleX}

Considerando a equação de estimação (3.12), o vetor perturbado na variável resposta referente à $i$-ésima unidade experimental é dado por

$$
\mathbf{b}_{\omega i}=\left(\begin{array}{c}
\mathbf{b}_{1 \omega i} \\
\mathbf{b}_{2 \omega i}
\end{array}\right)
$$

sendo $\mathbf{b}_{1 \omega i}=\left(\mathrm{b}_{1 \omega i 1}, \ldots, \mathrm{b}_{1 \omega i t}\right)^{\top}$ e $\mathbf{b}_{2 \omega i}=\left(\mathrm{b}_{2 \omega i 1}, \ldots, \mathrm{b}_{2 \omega i t}\right)^{\top}$, em que $\mathrm{b}_{1 \omega i j}$ é definido conforme a equação (6.13), cuja derivada com relação à $\omega_{i j}$ é dada em (6.14); e b b $_{2 \omega i j}=$ $\left[d\left(\mathrm{y}_{\omega i j} ; \mu_{i j}\right)-\phi_{i j}^{-1}\right]$, cuja derivada com relação à $\omega_{i j}$ é dada por

$$
\frac{\partial \mathrm{b}_{2 \omega i j}}{\partial \omega_{i j}}=\frac{\partial d\left(\mathrm{y}_{\omega i j} ; \mu_{i j}\right)}{\partial \omega_{i j}}=s_{i j} \frac{\left(\mathrm{y}_{\omega i j}-\mu_{i j}\right)\left(\mathrm{y}_{\omega i j}-2 \mathrm{y}_{\omega i j} \mu_{i j}+\mu_{i j}\right)}{\mu_{i j}^{2}\left(1-\mu_{i j}\right)^{2} \mathrm{y}_{\omega i j}^{2}\left(1-\mathrm{y}_{\omega i j}\right)^{2}}=s_{i j} \rho_{i j},
$$

com $i=1, \ldots, n$ e $j=1, \ldots, t$.

Logo, matricialmente temos

$$
\mathcal{B}=\left(\begin{array}{c}
\mathcal{S} \Xi \\
\mathcal{S} \mathcal{R}
\end{array}\right)
$$

sendo $\mathcal{S}$ e $\boldsymbol{\Xi}$ descritos em $(6.16)$ e $\mathcal{R}=\operatorname{diag}\left(\mathcal{R}_{1}, \ldots, \mathcal{R}_{n}\right)$, em que $\mathcal{R}_{i}=\operatorname{diag}\left(\rho_{i 1}, \ldots, \rho_{i t}\right)$, com $i=1, \ldots, n$.

\section{Modelos Lineares Generalizados}

Considerando a equação de estimação (4.10), o vetor perturbado na variável resposta referente à $i$-ésima unidade experimental é dado por

$$
\mathbf{b}_{\omega i}=\left(\begin{array}{c}
\mathbf{b}_{1 \omega i} \\
\mathbf{b}_{2 \omega i}
\end{array}\right)
$$

sendo $\mathbf{b}_{1 \omega i}=\left(b_{1 \omega i 1}, \ldots, b_{1 \omega i t}\right)^{\top}$ e $\mathbf{b}_{2 \omega i}=\left(b_{2 \omega i 1}, \ldots, b_{2 \omega i t}\right)^{\top}$, em que $b_{1 \omega i j}$ é definido conforme equação (6.17), cuja derivada com relação à $\omega_{i j}$ é dada em (6.18); e b bijij $=$ $\left[\mathrm{y}_{\omega i j} \pi_{i j}-b\left(\pi_{i j}\right)+\dot{c}\left(\mathrm{y}_{\omega i j} ; \phi_{i j}\right)\right]$, cuja derivada com relação à $\omega_{i j}$ é dada por

$$
\frac{\partial \mathrm{b}_{2 \omega i j}}{\partial \omega_{i j}}=s_{i j} \pi_{i j}+\frac{\partial \dot{c}\left(\mathrm{y}_{\omega i j} ; \phi_{i j}\right)}{\partial \omega_{i j}}
$$


com $i=1, \ldots, n$ e $j=1, \ldots, t$. A Tabela C.4 apresenta a derivada de $\dot{c}\left(\mathrm{y}_{\omega} ; \phi\right)$ com relação à $\omega$ para algumas distribuições pertencentes à família exponencial.

Logo, matricialmente temos

$$
\mathcal{B}=\left(\begin{array}{c}
\mathcal{S} \\
\mathcal{B}_{2}
\end{array}\right)
$$

sendo $\mathcal{S}$ descrito em $(6.19)$ e $\mathcal{B}_{2}=\operatorname{diag}\left(\mathcal{B}_{21}, \ldots, \mathcal{B}_{2 n}\right)$, em que $\mathcal{B}_{2 i}=\operatorname{diag}\left(\partial \mathrm{b}_{2 \omega i 1} / \partial \omega_{i 1}, \ldots\right.$, $\left.\partial \mathrm{b}_{2 \omega i t} / \partial \omega_{i t}\right), \operatorname{com} i=1, \ldots, n$.

\subsubsection{Perturbação individual das covariáveis}

Modificaremos a $k$-ésima coluna da matriz de covariáveis $\mathbf{X}$ conforme proposta apresentada na equação (6.20), para $k=2, \ldots, p$. Assim, perturbando a equação (6.40), obtemos

$$
\Psi_{2}(\boldsymbol{\theta} \mid \boldsymbol{\omega})=\mathbf{M}_{\omega}^{\top} \boldsymbol{\Lambda}_{\omega} \Omega_{\omega}^{-1} \mathbf{b}_{\omega}
$$

em que o índice $\omega$ indica que as matrizes $\mathbf{M}, \boldsymbol{\Lambda}$ e $\boldsymbol{\Omega}$ e o vetor $\mathbf{b}$ dependem, de alguma forma, da perturbação definida em (6.20). Logo, a matriz definida em (6.2) pode ser expressa por

$$
\boldsymbol{\Delta}=\mathbf{M}_{\omega}^{\top} \boldsymbol{\Lambda}_{\omega}\left[\boldsymbol{\Omega}_{\omega}^{-1} \frac{\partial \mathbf{b}_{\omega}}{\partial \boldsymbol{\omega}^{\top}}+\frac{\partial \boldsymbol{\Omega}_{\omega}^{-1}}{\partial \boldsymbol{\omega}^{\top}} \mathbf{B}_{\omega}\right]+\left[\mathbf{M}_{\omega}^{\top} \frac{\partial \boldsymbol{\Lambda}_{\omega}}{\partial \boldsymbol{\omega}^{\top}}+\frac{\partial \mathbf{M}_{\omega}^{\top}}{\partial \boldsymbol{\omega}^{\top}} \boldsymbol{\Lambda}_{\omega}\right] \boldsymbol{\Omega}_{\omega}^{-1} \mathbf{B}_{\omega},
$$

em que $\mathbf{B}_{\omega}$ é a matriz definida em (6.43) perturbada conforme esquema (6.20), as derivadas de $\boldsymbol{\Lambda}_{\omega}, \boldsymbol{\Omega}_{\omega}^{-1}$ e $\mathbf{b}_{\omega}$ todas com relação à $\boldsymbol{\omega}^{\top}$ são dadas, respectivamente, em (6.22), (6.21) e (6.23). Essas derivadas tomam forma de acordo com a definição da equação $\Psi_{2}(\boldsymbol{\theta} \mid \boldsymbol{\omega})$ que, nesta parte, pode ser a partir das equações de estimação (2.13), (3.12) e (4.10). Já a derivada de $\mathbf{M}_{\omega}^{\top}$ com relação à $\boldsymbol{\omega}^{\top}$ depende de como a matriz $\mathbf{Q}$, matriz de covariáveis para modelagem da precisão, está definida com relação à matriz $\mathbf{X}$, matriz de covariáveis que modelam a média.

Conforme proposta de Ospina (2007), quando os parâmetros de posição e de precisão são modelados simultaneamente, destacamos quatro cenários que relacionam as matrizes que modelam a média e a precisão:

1. $\mathbf{X} \neq \mathbf{Q}$, matrizes totalmente diferentes;

2. $\mathbf{X}=\mathbf{Q}$, matrizes totalmente iguais;

3. $\mathbf{X} \neq \mathbf{Q}$, com a $k^{\prime}$-ésima coluna da matriz $\mathbf{Q}$ igual a $k$-ésima coluna da matriz $\mathbf{X}$; e 
4. $\mathbf{X} \neq \mathbf{Q}$, com a $k^{\prime}$-ésima coluna da matriz $\mathbf{Q}$ função da $k$-ésima coluna da matriz $\mathbf{X}$.

A seguir, para cada um desses quatro cenários, apresentamos as medidas de influência local com perturbação em uma covariável.

\subsubsection{Matriz $\mathrm{Q}$ totalmente diferente da matriz $\mathrm{X}$}

Considerando que a matriz de covariáveis $\mathbf{Q}$ que modela a precisão é totalmente diferente da matriz $\mathbf{X}$, a qual tem a $k$-ésima coluna perturbada segundo o esquema definido em (6.20), a matriz de covariáveis $\mathbf{M}$ modificada é dada por

$$
\mathbf{M}_{\omega}^{\top}=\left(\begin{array}{cc}
\mathbf{X}_{\omega}^{\top} & \mathbf{0} \\
\mathbf{0} & \mathbf{Q}^{\top}
\end{array}\right)
$$

em que o índice $\omega$ indica que a matriz $\mathbf{X}$ depende da perturbação definida em (6.20). Logo, temos

$$
\frac{\partial \mathbf{M}_{\omega}^{\top}}{\partial \boldsymbol{\omega}^{\top}}=\left(\begin{array}{cc}
\frac{\partial \mathbf{X}_{\omega}^{\top}}{\partial \boldsymbol{\omega}^{\top}} & \mathbf{0} \\
\mathbf{0} & \mathbf{0}
\end{array}\right)
$$

sendo uma matriz $(p+q) \times 2 N$, em que a derivada de $\mathbf{X}_{\omega}^{\top}$ com relação à $\boldsymbol{\omega}^{\top}$ é uma matriz $p \times N$ de zeros exceto a $k$-ésima linha que é composta pela constante $s_{x_{k}}$, com $N=n t$.

A seguir, apresentamos as derivadas de $\boldsymbol{\Lambda}_{\omega}, \Omega_{\omega}$ e $\mathbf{b}_{\omega}$ todas com relação à $\boldsymbol{\omega}^{\top}$, quando consideramos cada uma das três equações de estimação: (2.13), (3.12) e (4.10).

\section{BETA}

De acordo com a equação de estimação definida em (2.13), temos

$$
\boldsymbol{\Lambda}_{\omega i}=\left(\begin{array}{c}
\mathbf{G}_{\omega i} \boldsymbol{\Phi}_{i} \mathbf{A}_{\omega i} \\
\mathbf{F}_{i} \mathbf{C}_{\omega i}
\end{array}\right), \quad \boldsymbol{\Omega}_{\omega i}=\mathbf{A}_{\omega i}^{1 / 2} \mathbf{R}(\boldsymbol{\alpha}) \mathbf{A}_{\omega i}^{1 / 2} \quad \text { e } \quad \mathbf{b}_{\omega i}=\mathbf{y}_{i}^{*}-\boldsymbol{\mu}_{\omega i}^{*}
$$

sendo $\mathbf{G}_{\omega i}=\operatorname{diag}\left(\partial g^{-1}\left(\eta_{\omega i 1}\right) / \partial \eta_{\omega i 1}, \ldots, \partial g^{-1}\left(\eta_{\omega i t}\right) / \partial \eta_{\omega i t}\right), \mathbf{A}_{\omega i}=\operatorname{diag}\left(\mathrm{a}_{\omega i 1}, \ldots, \mathrm{a}_{\omega i t}\right)$, $\mathbf{C}_{\omega i}=\operatorname{diag}\left(\mathrm{c}_{\omega i 1}, \ldots, \mathrm{c}_{\omega i t}\right)$ e $\boldsymbol{\mu}_{\omega i}^{*}=\left(\mu_{\omega i 1}^{*}, \ldots, \mu_{\omega i t}^{*}\right)^{\top}$, em que $\mathrm{a}_{\omega i j}=\psi^{\prime}\left(\mu_{\omega i j} \phi_{i j}\right)+\psi^{\prime}((1-$ $\left.\left.\mu_{\omega i j}\right) \phi_{i j}\right), c_{\omega i j}=\mu_{\omega i j} \psi^{\prime}\left(\mu_{\omega i j} \phi_{i j}\right)-\left(1-\mu_{\omega i j}\right) \psi^{\prime}\left(\left(1-\mu_{\omega i j}\right) \phi_{i j}\right)$ e $\mu_{\omega i j}^{*}=\psi\left(\mu_{\omega i j} \phi_{i j}\right)-\psi((1-$ $\left.\left.\mu_{\omega i j}\right) \phi_{i j}\right), \operatorname{com} i=1, \ldots, n$ e $j=1, \ldots, t$. Assim, da Seção B.2.1.1 do Apêndice B, obtemos

$$
\begin{aligned}
& \frac{\partial \boldsymbol{\Lambda}_{\omega i}}{\partial \boldsymbol{\omega}_{i}^{\top}}=\beta_{k} s_{x_{k}}\left(\begin{array}{c}
\boldsymbol{\Phi}_{i}\left[\dot{\mathbf{G}}_{\omega i} \mathbf{A}_{\omega i}+\mathbf{G}_{\omega i}^{2} \mathcal{E}_{1 \omega i}\right] \\
\mathbf{F}_{i} \mathbf{G}_{\omega i}\left[\mathbf{A}_{\omega i}+\boldsymbol{\Phi}_{i} \mathcal{E}_{2 \omega i}\right]
\end{array}\right), \\
& \frac{\partial \boldsymbol{\Omega}_{\omega i}}{\partial \boldsymbol{\omega}_{i}^{\top}}=\frac{1}{2} \beta_{k} s_{x_{k}}\left[\mathbf{A}_{\omega i}^{1 / 2} \mathbf{R}(\boldsymbol{\alpha}) \mathcal{A}_{\omega i}+\mathcal{A}_{\omega i} \mathbf{R}(\boldsymbol{\alpha}) \mathbf{A}_{\omega i}^{1 / 2}\right], \quad \mathrm{e} \\
& \frac{\partial \mathbf{b}_{\omega i}}{\partial \boldsymbol{\omega}_{i}^{\top}}=-\beta_{k} s_{x_{k}} \mathbf{G}_{\omega i} \boldsymbol{\Phi}_{i} \mathbf{A}_{\omega i}
\end{aligned}
$$


sendo $\dot{\mathbf{G}}_{\omega i}=\operatorname{diag}\left(\partial^{2} g^{-1}\left(\eta_{\omega i 1}\right) / \partial \eta_{\omega i 1}^{2}, \ldots, \partial^{2} g^{-1}\left(\eta_{\omega i t}\right) / \partial \eta_{\omega i t}^{2}\right), \mathcal{A}_{\omega i}=\mathbf{A}_{\omega i}^{-1 / 2} \mathbf{G}_{\omega i} \mathcal{E}_{1 \omega i}, \mathcal{E}_{1 \omega i}=$ $\operatorname{diag}\left(\mathcal{E}_{1 \omega i 1}, \ldots, \mathcal{E}_{1 \omega i t}\right)$ e $\mathcal{E}_{2 \omega i}=\operatorname{diag}\left(\mathcal{E}_{2 \omega i 1}, \ldots, \mathcal{E}_{2 \omega i t}\right)$, em que

$$
\mathcal{E}_{1 \omega i j}=\phi_{i j}\left[\psi^{\prime \prime}\left(\mu_{\omega i j} \phi_{i j}\right)-\psi^{\prime \prime}\left(\left(1-\mu_{\omega i j}\right) \phi_{i j}\right)\right]
$$

e

$$
\mathcal{E}_{2 \omega i j}=\left[\mu_{\omega i j} \psi^{\prime \prime}\left(\mu_{\omega i j} \phi_{i j}\right)+\left(1-\mu_{\omega i j}\right) \psi^{\prime \prime}\left(\left(1-\mu_{\omega i j}\right) \phi_{i j}\right)\right]
$$

$\operatorname{com} i=1, \ldots, n$ e $j=1, \ldots, t$.

\section{SimPLEX}

De acordo com a equação de estimação definida em (3.12), temos

$$
\boldsymbol{\Lambda}_{\omega i}=\left(\begin{array}{cc}
\mathbf{G}_{\omega i} \boldsymbol{\Phi}_{i} \mathbf{A}_{\omega i} & \mathbf{0} \\
\mathbf{0} & \mathbf{F}_{i}
\end{array}\right), \boldsymbol{\Omega}_{\omega i}=\left(\begin{array}{cc}
\mathbf{A}_{\omega i}^{1 / 2} \mathbf{R}(\boldsymbol{\alpha}) \mathbf{A}_{\omega i}^{1 / 2} & \mathbf{0} \\
\mathbf{0} & \mathbf{D}_{i}
\end{array}\right) \text { e } \mathbf{b}_{\omega i}=\left(\begin{array}{c}
\mathbf{u}_{\omega i} \\
\mathbf{b}_{2 \omega i}
\end{array}\right)
$$

sendo $\mathbf{G}_{\omega i}=\operatorname{diag}\left(\partial g^{-1}\left(\eta_{\omega i 1}\right) / \partial \eta_{\omega i 1}, \ldots, \partial g^{-1}\left(\eta_{\omega i t}\right) / \partial \eta_{\omega i t}\right), \mathbf{A}_{\omega i}=\operatorname{diag}\left(\mathrm{a}_{\omega i 1}, \ldots, \mathrm{a}_{\omega i t}\right)$, $\mathbf{u}_{\omega i}=\left(\mathrm{u}_{\omega i 1}, \ldots, \mathrm{u}_{\omega i t}\right)^{\top}$ e $\mathbf{b}_{2 \omega i}=\left(\mathrm{b}_{2 \omega i 1}, \ldots, \mathrm{b}_{2 \omega i t}\right)^{\top}$, em que $\mathrm{u}_{\omega i j}$ é definido em (6.27), $\mathrm{a}_{\omega i j}=\phi_{i j}^{-1}\left[3 \phi_{i j}^{-1} / \mu_{\omega i j}\left(1-\mu_{\omega i j}\right)+1 / \mu_{\omega i j}^{3}\left(1-\mu_{\omega i j}\right)^{3}\right]$ e $\mathrm{b}_{2 \omega i j}=\left[d\left(\mathrm{y}_{i j} ; \mu_{\omega i j}\right)-\phi_{i j}^{-1}\right]$, com $i=1, \ldots, n$ e $j=1, \ldots, t$. Assim, da Seção B.2.1.1 do Apêndice B, obtemos

$$
\begin{aligned}
\frac{\partial \boldsymbol{\Lambda}_{\omega i}}{\partial \boldsymbol{\omega}_{i}^{\top}} & =\beta_{k} s_{x_{k}}\left(\begin{array}{cc}
\boldsymbol{\Phi}_{i}\left[\dot{\mathbf{G}}_{\omega i} \mathbf{A}_{\omega i}+\mathbf{G}_{\omega i}^{2} \mathcal{E}_{1 \omega i}\right] & \mathbf{0} \\
\mathbf{0} & \mathbf{0}
\end{array}\right) \\
\frac{\partial \boldsymbol{\Omega}_{\omega i}}{\partial \boldsymbol{\omega}_{i}^{\top}} & =\frac{1}{2} \beta_{k} s_{x_{k}}\left(\begin{array}{cc}
\boldsymbol{\Phi}_{i}\left[\mathbf{A}_{\omega i}^{1 / 2} \mathbf{R}(\boldsymbol{\alpha}) \mathcal{A}_{\omega i}+\mathcal{A}_{\omega i} \mathbf{R}(\boldsymbol{\alpha}) \mathbf{A}_{\omega i}^{1 / 2}\right] & \mathbf{0} \\
\mathbf{0} & \mathbf{0}
\end{array}\right) \text { e } \\
\frac{\partial \mathbf{b}_{\omega i}}{\partial \boldsymbol{\omega}_{i}^{\top}} & =\beta_{k} s_{x_{k}}\left(\begin{array}{c}
\mathbf{G}_{\omega i} \mathcal{U}_{\omega i} \\
-2 \mathbf{G}_{\omega i} \operatorname{diag}\left(\mathbf{u}_{\omega i}\right)
\end{array}\right)
\end{aligned}
$$

sendo $\quad \dot{\mathbf{G}}_{\omega i}=\operatorname{diag}\left(\partial^{2} g^{-1}\left(\eta_{\omega i 1}\right) / \partial \eta_{\omega i 1}^{2}, \ldots, \partial^{2} g^{-1}\left(\eta_{\omega i t}\right) / \partial \eta_{\omega i t}^{2}\right), \quad \mathcal{A}_{\omega i}=\mathbf{A}_{\omega i}^{-1 / 2} \mathbf{G}_{\omega i} \mathcal{E}_{1 \omega i}$, $\mathcal{E}_{1 \omega i}=\operatorname{diag}\left(\mathcal{E}_{1 \omega i 1}, \ldots, \mathcal{E}_{1 \omega i t}\right)$ e $\mathcal{U}_{\omega i}=\operatorname{diag}\left(\mathcal{U}_{\omega i 1}, \ldots, \mathcal{U}_{\omega i t}\right)$, em que $\mathcal{U}_{\omega i j}$ está definido em (6.29) e

$$
\mathcal{E}_{1 \omega i j}=-3 \phi_{i j}^{-1}\left[\frac{\phi_{i j}^{-1}\left(1-2 \mu_{\omega i j}\right)}{\left(\mu_{\omega i j}\left(1-\mu_{\omega i j}\right)\right)^{2}}+\frac{\mu_{\omega i j}^{2}\left(1-\mu_{\omega i j}\right)^{3}-\mu_{\omega i j}^{3}\left(1-\mu_{\omega i j}\right)^{2}}{\left(\mu_{\omega i j}^{3}\left(1-\mu_{\omega i j}\right)^{3}\right)^{2}}\right],
$$

$\operatorname{com} i=1, \ldots, n$ e $j=1, \ldots, t$. 
Modelos Lineares Generalizados

De acordo com a equação de estimação definida em (4.10), temos

$$
\boldsymbol{\Lambda}_{\omega i}=\left(\begin{array}{cc}
\mathbf{G}_{\omega i} & \mathbf{0} \\
\mathbf{0} & \mathbf{F}_{i} \mathrm{E}\left(\ddot{\mathbf{c}}_{i}\right)
\end{array}\right), \boldsymbol{\Omega}_{\omega i}=\left(\begin{array}{cc}
\boldsymbol{\Sigma}_{\omega i} & \mathbf{0} \\
\mathbf{0} & \boldsymbol{\Pi}_{\omega i} \boldsymbol{\Sigma}_{\omega i} \boldsymbol{\Pi}_{\omega i}
\end{array}\right) \text { e } \mathbf{b}_{\omega i}=\left(\begin{array}{c}
\mathbf{y}_{i}-\boldsymbol{\mu}_{\omega i} \\
\mathbf{b}_{2 \omega i}
\end{array}\right)
$$

sendo $\mathbf{G}_{\omega i}=\operatorname{diag}\left(\partial g^{-1}\left(\eta_{\omega i 1}\right) / \partial \eta_{\omega i 1}, \ldots, \partial g^{-1}\left(\eta_{\omega i t}\right) / \partial \eta_{\omega i t}\right), \quad \boldsymbol{\Pi}_{\omega i}=\operatorname{diag}\left(\pi_{\omega i 1}, \ldots, \pi_{\omega i t}\right)$, $\boldsymbol{\Sigma}_{\omega i}=\left(\boldsymbol{\Phi}_{i}^{-1} \mathbf{A}_{\omega i}\right)^{1 / 2} \mathbf{R}(\boldsymbol{\alpha})\left(\mathbf{A}_{\omega i} \boldsymbol{\Phi}_{i}^{-1}\right)^{1 / 2}, \mathbf{A}_{\omega i}=\operatorname{diag}\left(\mathrm{a}_{\omega i 1}, \ldots, \mathrm{a}_{\omega i t}\right), \boldsymbol{\mu}_{\omega i}=\left(\mu_{\omega i 1}, \ldots, \mu_{\omega i t}\right)^{\top}$ e $\mathbf{b}_{2 \omega i}=\left(\mathrm{b}_{2 \omega i 1}, \ldots, \mathrm{b}_{2 \omega i t}\right)^{\top}$, em que $\mathrm{a}_{\omega i j}=v\left(\mu_{\omega i j}\right)$ e $\mathrm{b}_{2 \omega i j}=\left[\mathrm{y}_{i j} \varphi_{i j}-b\left(\varphi_{i j}\right)+\dot{c}\left(\mathrm{y}_{i j} ; \phi_{i j}\right)\right]$, com $i=1, \ldots, n$ e $j=1, \ldots, t$. Assim, obtemos

$$
\begin{aligned}
& \frac{\partial \boldsymbol{\Lambda}_{\omega i}}{\partial \boldsymbol{\omega}_{i}^{\top}}=\beta_{k} s_{x_{k}}\left(\begin{array}{cc}
\dot{\mathbf{G}}_{\omega i} & \mathbf{0} \\
\mathbf{0} & \mathbf{0}
\end{array}\right) \\
& \frac{\partial \boldsymbol{\Omega}_{\omega i}}{\partial \boldsymbol{\omega}_{i}^{\top}}=\left(\begin{array}{cc}
\frac{\partial \boldsymbol{\Sigma}_{\omega i}}{\partial \boldsymbol{\omega}_{i}^{\top}} & \mathbf{0} \\
\mathbf{0} & \frac{\partial\left[\boldsymbol{\Pi}_{\omega i} \boldsymbol{\Sigma}_{\omega i} \boldsymbol{\Pi}_{\omega i}\right]}{\partial \boldsymbol{\omega}_{i}^{\top}}
\end{array}\right) \mathrm{e} \\
& \frac{\partial \mathbf{b}_{\omega i}}{\partial \boldsymbol{\omega}_{i}^{\top}}=\beta_{k} s_{x_{k}}\left(\begin{array}{c}
-\mathbf{G}_{\omega i} \\
\mathbf{G}_{\omega i} \mathcal{B}_{2 \omega i}
\end{array}\right)
\end{aligned}
$$

sendo

$$
\begin{aligned}
& \frac{\partial \boldsymbol{\Sigma}_{\omega i}}{\partial \boldsymbol{\omega}_{i}^{\top}}=\frac{1}{2} \beta_{k} s_{x_{k}}\left[\left(\boldsymbol{\Phi}_{i}^{-1} \mathbf{A}_{\omega i}\right)^{1 / 2} \mathbf{R}(\boldsymbol{\alpha}) \mathcal{A}_{\omega i} \boldsymbol{\Phi}_{i}^{-1 / 2}+\boldsymbol{\Phi}_{i}^{-1 / 2} \mathcal{A}_{\omega i} \mathbf{R}(\boldsymbol{\alpha})\left(\mathbf{A}_{\omega i} \boldsymbol{\Phi}_{i}^{-1}\right)^{1 / 2}\right] \\
& \frac{\partial\left[\boldsymbol{\Pi}_{\omega i} \boldsymbol{\Sigma}_{\omega i} \boldsymbol{\Pi}_{\omega i}\right]}{\partial \boldsymbol{\omega}_{i}^{\top}}=\boldsymbol{\Pi}_{\omega i} \boldsymbol{\Sigma}_{\omega i} \frac{\partial \boldsymbol{\Pi}_{\omega i}}{\partial \boldsymbol{\omega}_{i}^{\top}}+\boldsymbol{\Pi}_{\omega i} \frac{\partial \boldsymbol{\Sigma}_{\omega i}}{\partial \boldsymbol{\omega}_{i}^{\top}} \boldsymbol{\Pi}_{\omega i}+\frac{\partial \boldsymbol{\Pi}_{\omega i}}{\partial \boldsymbol{\omega}_{i}^{\top}} \boldsymbol{\Sigma}_{\omega i} \boldsymbol{\Pi}_{\omega i}, \\
& \frac{\partial \boldsymbol{\Pi}_{\omega i}}{\partial \boldsymbol{\omega}_{i}^{\top}}=\beta_{k} s_{x_{k}} \dot{\mathbf{G}}_{\omega i} \frac{\partial \boldsymbol{\Pi}_{\omega i}}{\partial \boldsymbol{\mu}_{\omega i}^{\top}}
\end{aligned}
$$

$\dot{\mathbf{G}}_{\omega i}=\operatorname{diag}\left(\partial^{2} g^{-1}\left(\eta_{\omega i 1}\right) / \partial \eta_{\omega i 1}^{2}, \ldots, \partial^{2} g^{-1}\left(\eta_{\omega i t}\right) / \partial \eta_{\omega i t}^{2}\right), \mathcal{A}_{\omega i}=\mathbf{A}_{\omega i}^{-1 / 2} \mathbf{G}_{\omega i} \dot{\boldsymbol{\Upsilon}}_{\omega i}, \partial \mathbf{\Pi}_{\omega i} / \partial \boldsymbol{\mu}_{\omega i}^{\top}=$ $\operatorname{diag}\left(\partial \varphi_{\omega i 1} / \partial \mu_{\omega i 1}, \ldots, \partial \varphi_{\omega i t} / \partial \mu_{\omega i t}\right) \quad$ e $\quad \mathcal{B}_{2 \omega i}=\operatorname{diag}\left(\mathcal{B}_{2 \omega i 1}, \ldots, \mathcal{B}_{2 \omega i t}\right)$, em que $\dot{\boldsymbol{\Upsilon}}_{\omega i}=$ $\operatorname{diag}\left(\partial v\left(\mu_{\omega i 1}\right) / \partial \mu_{\omega i 1}, \ldots, \partial v\left(\mu_{\omega i t}\right) / \partial \mu_{\omega i t}\right)$ e $\mathcal{B}_{2 \omega i j}=\mathrm{y}_{i j} \partial \varphi_{\omega i j} / \partial \mu_{\omega i j}-\left[\left(\partial b\left(\varphi_{\omega i j}\right) / \partial \varphi_{\omega i j}\right)\right.$ $\left.\left(\partial \varphi_{\omega i j} / \partial \mu_{\omega i j}\right)\right]$, com $i=1, \ldots, n$ e $j=1, \ldots, t$. Ressaltamos que as derivadas envolvendo $\varphi_{\omega i j}$ dependem da distribuição adotada para $\mathrm{y}_{i j}$, cuja densidade deve satisfazer (4.6). A Tabela C.4 apresenta essas derivadas para algumas distribuições pertencentes à família exponencial.

\subsubsection{Matriz Q totalmente igual à matriz X}

Considerando que a matriz de covariáveis $\mathbf{Q}$ que modela a precisão é totalmente igual à matriz $\mathbf{X}$, a qual tem a $k$-ésima coluna perturbada segundo o esquema definido em 
(6.20), a matriz de covariáveis $\mathbf{M}$ modificada é dada por

$$
\mathbf{M}_{\omega}^{\top}=\left(\begin{array}{cc}
\mathbf{X}_{\omega}^{\top} & \mathbf{0} \\
\mathbf{0} & \mathbf{Q}_{\omega}^{\top}
\end{array}\right)=\left(\begin{array}{cc}
\mathbf{X}_{\omega}^{\top} & \mathbf{0} \\
\mathbf{0} & \mathbf{X}_{\omega}^{\top}
\end{array}\right)
$$

em que o índice $\omega$ indica que a matriz $\mathbf{X}$ depende da perturbação definida em (6.20). Logo, temos

$$
\frac{\partial \mathbf{M}_{\omega}^{\top}}{\partial \boldsymbol{\omega}^{\top}}=\left(\begin{array}{cc}
\frac{\partial \mathbf{X}_{\omega}^{\top}}{\partial \boldsymbol{\omega}^{\top}} & \mathbf{0} \\
\mathbf{0} & \frac{\partial \mathbf{X}_{\omega}^{\top}}{\partial \boldsymbol{\omega}^{\top}}
\end{array}\right)
$$

sendo uma matriz $(2 p) \times 2 N$, em que a derivada de $\mathbf{X}_{\omega}^{\top}$ com relação à $\boldsymbol{\omega}^{\top}$ é uma matriz $p \times N$ de zeros exceto a $k$-ésima linha que é composta pela constante $s_{x_{k}}$, com $N=n t$.

A seguir, apresentamos as derivadas de $\boldsymbol{\Lambda}_{\omega}, \boldsymbol{\Omega}_{\omega}$ e $\mathbf{b}_{\omega}$ todas com relação à $\boldsymbol{\omega}^{\top}$, quando consideramos cada uma das três equações de estimação: (2.13), (3.12) e (4.10).

\section{BETA}

De acordo com a equação de estimação definida em (2.13), temos

$$
\boldsymbol{\Lambda}_{\omega i}=\left(\begin{array}{c}
\mathbf{G}_{\omega i} \boldsymbol{\Phi}_{\omega i} \mathbf{A}_{\omega i} \\
\mathbf{F}_{\omega i} \mathbf{C}_{\omega i}
\end{array}\right), \quad \boldsymbol{\Omega}_{\omega i}=\mathbf{A}_{\omega i}^{1 / 2} \mathbf{R}(\boldsymbol{\alpha}) \mathbf{A}_{\omega i}^{1 / 2} \quad \text { e } \quad \mathbf{b}_{\omega i}=\mathbf{y}_{i}^{*}-\boldsymbol{\mu}_{\omega i}^{*}
$$

sendo $\mathbf{G}_{\omega i}=\operatorname{diag}\left(\partial g^{-1}\left(\eta_{\omega i 1}\right) / \partial \eta_{\omega i 1}, \ldots, \partial g^{-1}\left(\eta_{\omega i t}\right) / \partial \eta_{\omega i t}\right), \boldsymbol{\Phi}_{\omega i}=\operatorname{diag}\left(\phi_{\omega i 1}, \ldots, \phi_{\omega i t}\right)$, $\mathbf{A}_{\omega i}=\operatorname{diag}\left(\mathrm{a}_{\omega i 1}, \ldots, \mathrm{a}_{\omega i t}\right), \quad \mathbf{F}_{\omega i}=\operatorname{diag}\left(\partial f^{-1}\left(\delta_{\omega i 1}\right) / \partial \delta_{\omega i 1}, \ldots, \partial f^{-1}\left(\delta_{\omega i t}\right) / \partial \delta_{\omega i t}\right), \quad \mathbf{C}_{\omega i}=$ $\operatorname{diag}\left(\mathrm{c}_{\omega i 1}, \ldots, \mathrm{c}_{\omega i t}\right)$ e $\boldsymbol{\mu}_{\omega i}^{*}=\left(\mu_{\omega i 1}^{*}, \ldots, \mu_{\omega i t}^{*}\right)^{\top}$, em que $\mathrm{a}_{\omega i j}=\psi^{\prime}\left(\mu_{\omega i j} \phi_{\omega i j}\right)+\psi^{\prime}((1-$ $\left.\left.\mu_{\omega i j}\right) \phi_{\omega i j}\right), \quad c_{\omega i j}=\mu_{\omega i j} \psi^{\prime}\left(\mu_{\omega i j} \phi_{\omega i j}\right)-\left(1-\mu_{\omega i j}\right) \psi^{\prime}\left(\left(1-\mu_{\omega i j}\right) \phi_{\omega i j}\right)$ e $\mu_{\omega i j}^{*}=\psi\left(\mu_{\omega i j} \phi_{\omega i j}\right)-$ $\psi\left(\left(1-\mu_{\omega i j}\right) \phi_{\omega i j}\right), \operatorname{com} i=1, \ldots, n$ e $j=1, \ldots, t$. Assim, da Seção B.2.1.2 do Apêndice $\mathrm{B}$, obtemos

$$
\begin{aligned}
& \frac{\partial \boldsymbol{\Lambda}_{\omega i}}{\partial \boldsymbol{\omega}_{i}^{\top}}=\left(\begin{array}{c}
\beta_{k} s_{x_{k}} \boldsymbol{\Phi}_{\omega i}\left[\dot{\mathbf{G}}_{\omega i} \mathbf{A}_{\omega i}+\mathbf{G}_{\omega i}^{2} \mathcal{E}_{1 \omega i}\right]+\gamma_{k} s_{x_{k}} \mathbf{G}_{\omega i} \mathbf{F}_{\omega i}\left[\mathbf{A}_{\omega i}+\mathbf{\Phi}_{\omega i} \mathcal{E}_{2 \omega i}\right] \\
\beta_{k} s_{x_{k}} \mathbf{F}_{\omega i} \mathbf{C}_{\omega i}\left[\mathbf{A}_{\omega i}+\mathcal{M}_{\omega i} \mathcal{E}_{1 \omega i}\right]+\gamma_{k} s_{x_{k}}\left[\dot{\mathbf{F}}_{\omega i} \mathbf{C}_{\omega i}-\mathbf{F}_{\omega i}^{2}\left(\mathbf{I}_{t}-\mathcal{M}_{\omega i}\right) \mathcal{E}_{2 \omega i}\right]
\end{array}\right), \\
& \frac{\partial \boldsymbol{\Omega}_{\omega i}}{\partial \boldsymbol{\omega}_{i}^{\top}}=\frac{1}{2}\left[\mathbf{A}_{\omega i}^{1 / 2} \mathbf{R}(\boldsymbol{\alpha}) \mathcal{A}_{\omega i}+\mathcal{A}_{\omega i} \mathbf{R}(\boldsymbol{\alpha}) \mathbf{A}_{\omega i}^{1 / 2}\right] \mathrm{e} \\
& \frac{\partial \mathbf{b}_{\omega i}}{\partial \boldsymbol{\omega}_{i}^{\top}}=-\left[\beta_{k} s_{x_{k}} \mathbf{G}_{\omega i} \boldsymbol{\Phi}_{\omega i} \mathbf{A}_{\omega i}+\gamma_{k} s_{x_{k}} \mathbf{F}_{\omega i} \mathcal{E}_{3 \omega i}\right],
\end{aligned}
$$

sendo $\mathcal{A}_{\omega i}=\mathbf{A}_{\omega i}^{-1 / 2}\left[\beta_{k} s_{x_{k}} \mathbf{G}_{\omega i} \mathcal{E}_{1 \omega i}+\gamma_{k} s_{x_{k}} \mathbf{F}_{\omega i} \mathcal{E}_{2 \omega i}\right], \quad \mathcal{E}_{1 \omega i}=\operatorname{diag}\left(\mathcal{E}_{1 \omega i 1}, \ldots, \mathcal{E}_{1 \omega i t}\right), \mathcal{E}_{2 \omega i}=$ $\operatorname{diag}\left(\mathcal{E}_{2 \omega i 1}, \ldots, \mathcal{E}_{2 \omega i t}\right), \mathcal{E}_{3 \omega i}=\operatorname{diag}\left(\mathcal{E}_{3 \omega i 1}, \ldots, \mathcal{E}_{3 \omega i t}\right), \mathcal{M}_{\omega i}=\operatorname{diag}\left(\mu_{\omega i 1}, \ldots, \mu_{\omega i t}\right), \dot{\mathbf{G}}_{\omega i}=$ $\operatorname{diag}\left(\partial^{2} g^{-1}\left(\eta_{\omega i 1}\right) / \partial \eta_{\omega i 1}^{2}, \ldots, \partial^{2} g^{-1}\left(\eta_{\omega i t}\right) / \partial \eta_{\omega i t}^{2}\right), \quad \dot{\mathbf{F}}_{\omega i}=\operatorname{diag}\left(\partial^{2} f^{-1}\left(\delta_{\omega i 1}\right) / \partial \delta_{\omega i 1}^{2}, \ldots\right.$, 
$\left.\partial^{2} f^{-1}\left(\delta_{\omega i t}\right) / \partial \delta_{\omega i t}^{2}\right)$ e $\mathbf{I}_{t}$ uma matriz identidade de dimensão $t \times t$, em que

$$
\begin{aligned}
& \mathcal{E}_{1 \omega i j}=\phi_{\omega i j}\left[\psi^{\prime \prime}\left(\mu_{\omega i j} \phi_{\omega i j}\right)-\psi^{\prime \prime}\left(\left(1-\mu_{\omega i j}\right) \phi_{\omega i j}\right)\right], \\
& \mathcal{E}_{2 \omega i j}=\left[\mu_{\omega i j} \psi^{\prime \prime}\left(\mu_{\omega i j} \phi_{\omega i j}\right)+\left(1-\mu_{\omega i j}\right) \psi^{\prime \prime}\left(\left(1-\mu_{\omega i j}\right) \phi_{\omega i j}\right)\right],
\end{aligned}
$$

e

$$
\mathcal{E}_{3 \omega i j}=\left[\mu_{\omega i j} \psi^{\prime}\left(\mu_{\omega i j} \phi_{\omega i j}\right)-\left(1-\mu_{\omega i j}\right) \psi^{\prime}\left(\left(1-\mu_{\omega i j}\right) \phi_{\omega i j}\right)\right]
$$

$\operatorname{com} i=1, \ldots, n$ e $j=1, \ldots, t$.

\section{SimpleX}

De acordo com a equação de estimação definida em (3.12), temos

$$
\boldsymbol{\Lambda}_{\omega i}=\left(\begin{array}{cc}
\mathbf{G}_{\omega i} \boldsymbol{\Phi}_{\omega i} \mathbf{A}_{\omega i} & \mathbf{0} \\
\mathbf{0} & \mathbf{F}_{\omega i}
\end{array}\right), \boldsymbol{\Omega}_{\omega i}=\left(\begin{array}{cc}
\mathbf{A}_{\omega i}^{1 / 2} \mathbf{R}(\boldsymbol{\alpha}) \mathbf{A}_{\omega i}^{1 / 2} & \mathbf{0} \\
\mathbf{0} & \mathbf{D}_{\omega i}
\end{array}\right) \text { e } \mathbf{b}_{\omega i}=\left(\begin{array}{c}
\mathbf{u}_{\omega i} \\
\mathbf{b}_{2 \omega i}
\end{array}\right)
$$

sendo $\mathbf{G}_{\omega i}=\operatorname{diag}\left(\partial g^{-1}\left(\eta_{\omega i 1}\right) / \partial \eta_{\omega i 1}, \ldots, \partial g^{-1}\left(\eta_{\omega i t}\right) / \partial \eta_{\omega i t}\right), \boldsymbol{\Phi}_{\omega i}=\operatorname{diag}\left(\phi_{\omega i 1}, \ldots, \phi_{\omega i t}\right)$, $\mathbf{A}_{\omega i}=\operatorname{diag}\left(\mathrm{a}_{\omega i 1}, \ldots, \mathrm{a}_{\omega i t}\right), \mathbf{F}_{\omega i}=\operatorname{diag}\left(\partial f^{-1}\left(\delta_{\omega i 1}\right) / \partial \delta_{\omega i 1}, \ldots, \partial f^{-1}\left(\delta_{\omega i t}\right) / \partial \delta_{\omega i t}\right), \quad \mathbf{D}_{\omega i}=$ $\operatorname{diag}\left(2\left(\phi_{\omega i 1}^{-1}\right)^{2}, \ldots, 2\left(\phi_{\omega i t}^{-1}\right)^{2}\right), \quad \mathbf{u}_{\omega i}=\left(\mathrm{u}_{\omega i 1}, \ldots, \mathrm{u}_{\omega i t}\right)^{\top}$ e $\mathbf{b}_{2 \omega i}=\left(\mathrm{b}_{2 \omega i 1}, \ldots, \mathrm{b}_{2 \omega i t}\right)^{\top}, \mathrm{em}$

que $\mathrm{u}_{\omega i j}$ é definido em $(6.27), \mathrm{a}_{\omega i j}=\phi_{\omega i j}^{-1}\left[3 \phi_{\omega i j}^{-1} / \mu_{\omega i j}\left(1-\mu_{\omega i j}\right)+1 / \mu_{\omega i j}^{3}\left(1-\mu_{\omega i j}\right)^{3}\right]$ e $\mathrm{b}_{2 \omega i j}=\left[d\left(\mathrm{y}_{i j} ; \mu_{\omega i j}\right)-\phi_{\omega i j}^{-1}\right]$, com $i=1, \ldots, n$ e $j=1, \ldots, t$. Assim, da Seção B.2.1.2 do Apêndice B, obtemos

$$
\begin{aligned}
\frac{\partial \boldsymbol{\Lambda}_{\omega i}}{\partial \boldsymbol{\omega}_{i}^{\top}} & =\left(\begin{array}{cc}
\beta_{k} s_{x_{k}} \boldsymbol{\Phi}_{\omega i}\left[\dot{\mathbf{G}}_{\omega i} \mathbf{A}_{\omega i}+\mathbf{G}_{\omega i}^{2} \mathcal{E}_{1 \omega i}\right]-\gamma_{k} s_{x_{k}} \mathbf{G}_{\omega i} \mathbf{F}_{\omega i} \boldsymbol{\Phi}_{\omega i}^{2}\left[\mathbf{A}_{\omega i}+\mathbf{\Phi}_{\omega i} \mathcal{E}_{2 \omega i}\right] & \mathbf{0} \\
\mathbf{0} & \gamma_{k} s_{x_{k}} \dot{\mathbf{F}}_{\omega i}
\end{array}\right), \\
\frac{\partial \boldsymbol{\Omega}_{\omega i}}{\partial \boldsymbol{\omega}_{i}^{\top}} & =\left(\begin{array}{cc}
\frac{1}{2}\left[\mathbf{A}_{\omega i}^{1 / 2} \mathbf{R}(\boldsymbol{\alpha}) \mathcal{A}_{\omega i}+\mathcal{A}_{\omega i} \mathbf{R}(\boldsymbol{\alpha}) \mathbf{A}_{\omega i}^{1 / 2}\right] & \mathbf{0} \\
\mathbf{0} & 4 \gamma_{k} s_{x_{k}} \mathbf{F}_{\omega i} \boldsymbol{\Phi}_{\omega i}^{-1}
\end{array}\right) \\
\frac{\partial \mathbf{b}_{\omega i}}{\partial \boldsymbol{\omega}_{i}^{\top}} & =\left(\begin{array}{c}
\beta_{k} s_{x_{k}} \mathbf{G}_{\omega i} \mathcal{U}_{\omega i} \\
-\left[2 \beta_{k} s_{x_{k}} \mathbf{G}_{\omega i} \operatorname{diag}\left(\mathbf{u}_{\omega i}\right)+\gamma_{k} s_{x_{k}} \mathbf{F}_{\omega i}\right]
\end{array}\right),
\end{aligned}
$$

sendo $\mathcal{A}_{\omega i}=\mathbf{A}_{\omega i}^{-1 / 2}\left[\beta_{k} s_{x_{k}} \mathbf{G}_{\omega i} \mathcal{E}_{1 \omega i}-\gamma_{k} s_{x_{k}} \mathbf{F}_{\omega i} \boldsymbol{\Phi}_{\omega i}^{2} \mathcal{E}_{2 \omega i}\right], \quad \mathcal{E}_{1 \omega i}=\operatorname{diag}\left(\mathcal{E}_{1 \omega i 1}, \ldots, \mathcal{E}_{1 \omega i t}\right)$, $\mathcal{E}_{2 \omega i}=\operatorname{diag}\left(\mathcal{E}_{2 \omega i 1}, \ldots, \mathcal{E}_{2 \omega i t}\right), \quad \dot{\mathbf{G}}_{\omega i}=\operatorname{diag}\left(\partial^{2} g^{-1}\left(\eta_{\omega i 1}\right) / \partial \eta_{\omega i 1}^{2}, \ldots, \partial^{2} g^{-1}\left(\eta_{\omega i t}\right) / \partial \eta_{\omega i t}^{2}\right)$, $\dot{\mathbf{F}}_{\omega i}=\operatorname{diag}\left(\partial^{2} f^{-1}\left(\delta_{\omega i 1}\right) / \partial \delta_{\omega i 1}^{2}, \ldots, \partial^{2} f^{-1}\left(\delta_{\omega i t}\right) / \partial \delta_{\omega i t}^{2}\right)$ e $\mathcal{U}_{\omega i}=\operatorname{diag}\left(\mathcal{U}_{\omega i 1}, \ldots, \mathcal{U}_{\omega i t}\right)$, em que $\mathcal{U}_{\omega i j}$ está definido em (6.29),

$\mathrm{e}$

$$
\mathcal{E}_{1 \omega i j}=-3 \phi_{\omega i j}^{-1}\left[\frac{\phi_{\omega i j}^{-1}\left(1-2 \mu_{\omega i j}\right)}{\left(\mu_{\omega i j}\left(1-\mu_{\omega i j}\right)\right)^{2}}+\frac{\mu_{\omega i j}^{2}\left(1-\mu_{\omega i j}\right)^{3}-\mu_{\omega i j}^{3}\left(1-\mu_{\omega i j}\right)^{2}}{\left(\mu_{\omega i j}^{3}\left(1-\mu_{\omega i j}\right)^{3}\right)^{2}}\right]
$$

$$
\mathcal{E}_{2 \omega i j}=-\phi_{\omega i j}^{-2}\left[\frac{6 \phi_{\omega i j}^{-1}}{\mu_{\omega i j}\left(1-\mu_{\omega i j}\right)}+\frac{1}{\mu_{\omega i j}^{3}\left(1-\mu_{\omega i j}\right)^{3}}\right]
$$


$\operatorname{com} i=1, \ldots, n$ e $j=1, \ldots, t$.

\section{Modelos Lineares Generalizados}

De acordo com a equação de estimação definida em (4.10), temos

$\boldsymbol{\Lambda}_{\omega i}=\left(\begin{array}{cc}\mathbf{G}_{\omega i} & \mathbf{0} \\ \mathbf{0} & \mathbf{F}_{\omega i} \mathrm{E}\left(\ddot{\mathbf{c}}_{\omega i}\right)\end{array}\right), \boldsymbol{\Omega}_{\omega i}=\left(\begin{array}{cc}\boldsymbol{\Sigma}_{\omega i} & \mathbf{0} \\ \mathbf{0} & \boldsymbol{\Pi}_{\omega i} \Sigma_{\omega i} \boldsymbol{\Pi}_{\omega i}\end{array}\right)$ e $\mathbf{b}_{\omega i}=\left(\begin{array}{c}\mathbf{y}_{i}-\boldsymbol{\mu}_{\omega i} \\ \mathbf{b}_{2 \omega i}\end{array}\right)$

sendo $\mathbf{G}_{\omega i}=\operatorname{diag}\left(\partial g^{-1}\left(\eta_{\omega i 1}\right) / \partial \eta_{\omega i 1}, \ldots, \partial g^{-1}\left(\eta_{\omega i t}\right) / \partial \eta_{\omega i t}\right), \mathbf{F}_{\omega i}=\operatorname{diag}\left(\partial f^{-1}\left(\delta_{\omega i 1}\right) / \partial \delta_{\omega i 1}\right.$, $\left.\ldots, \partial f^{-1}\left(\delta_{\omega i t}\right) / \partial \delta_{\omega i t}\right), \boldsymbol{\Pi}_{\omega i}=\operatorname{diag}\left(\varphi_{\omega i 1}, \ldots, \varphi_{\omega i t}\right), \boldsymbol{\Sigma}_{\omega i}=\left(\boldsymbol{\Phi}_{\omega i}^{-1} \mathbf{A}_{\omega i}\right)^{1 / 2} \mathbf{R}(\boldsymbol{\alpha})\left(\mathbf{A}_{\omega i} \boldsymbol{\Phi}_{\omega i}^{-1}\right)^{1 / 2}$, $\mathbf{A}_{\omega i}=\operatorname{diag}\left(\mathrm{a}_{\omega i 1}, \ldots, \mathrm{a}_{\omega i t}\right), \ddot{\mathbf{c}}_{\omega i}=\operatorname{diag}\left(\ddot{c}_{\omega i 1}, \ldots, \ddot{c}_{\omega i t}\right), \boldsymbol{\mu}_{\omega i}=\left(\mu_{\omega i 1}, \ldots, \mu_{\omega i t}\right)^{\top}$ e $\mathbf{b}_{2 \omega i}=$ $\left(\mathrm{b}_{2 \omega i 1}, \ldots, \mathrm{b}_{2 \omega i t}\right)^{\top}$, em que $\mathrm{a}_{\omega i j}=v\left(\mu_{\omega i j}\right), \mathrm{b}_{2 \omega i j}=\left[\mathrm{y}_{i j} \varphi_{i j}-b\left(\varphi_{i j}\right)+\dot{c}\left(\mathrm{y}_{i j} ; \phi_{i j}\right)\right]$ e $\ddot{c}_{i j}$ é a derivada de $\dot{c}\left(\mathrm{y}_{i j} ; \phi_{i j}\right)$ com relação à $\phi_{i j}^{-1}, \operatorname{com} i=1, \ldots, n$ e $j=1, \ldots, t$. Assim, obtemos

$$
\begin{aligned}
& \frac{\partial \boldsymbol{\Lambda}_{\omega i}}{\partial \boldsymbol{\omega}_{i}^{\top}}=\left(\begin{array}{cc}
\beta_{k} s_{x_{k}} \dot{\mathbf{G}}_{\omega i} & \mathbf{0} \\
\mathbf{0} & \gamma_{k} s_{x_{k}}\left[\dot{\mathbf{F}}_{\omega i} \mathrm{E}\left(\ddot{\mathbf{c}}_{\omega i}\right)+\mathbf{F}_{\omega i}^{2} \dot{\mathrm{E}}\left(\ddot{\mathbf{c}}_{\omega i}\right)\right]
\end{array}\right), \\
& \frac{\partial \boldsymbol{\Omega}_{\omega i}}{\partial \boldsymbol{\omega}_{i}^{\top}}=\left(\begin{array}{cc}
\frac{\partial \boldsymbol{\Sigma}_{\omega i}}{\partial \boldsymbol{\omega}_{i}^{\top}} & \mathbf{0} \\
\mathbf{0} & \frac{\partial\left[\boldsymbol{\Pi}_{\omega i} \boldsymbol{\Sigma}_{\omega i} \boldsymbol{\Pi}_{\omega i}\right]}{\partial \boldsymbol{\omega}_{i}^{\top}}
\end{array}\right) \mathrm{e} \\
& \frac{\partial \mathbf{b}_{\omega i}}{\partial \boldsymbol{\omega}_{i}^{\top}}=\left(\begin{array}{c}
-\beta_{k} s_{x_{k}} \mathbf{G}_{\omega i} \\
\beta_{k} s_{x_{k}} \mathbf{G}_{\omega i} \mathcal{B}_{2 \omega i}+\gamma_{k} s_{x_{k}} \mathbf{F}_{\omega i} \ddot{\mathbf{c}}_{\omega i}
\end{array}\right),
\end{aligned}
$$

sendo

$$
\begin{aligned}
& \frac{\partial \boldsymbol{\Sigma}_{\omega i}}{\partial \boldsymbol{\omega}_{i}^{\top}}=\frac{1}{2}\left[\left(\boldsymbol{\Phi}_{\omega i}^{-1} \mathbf{A}_{\omega i}\right)^{1 / 2} \mathbf{R}(\boldsymbol{\alpha}) \mathcal{A}_{\omega i}+\mathcal{A}_{\omega i} \mathbf{R}(\boldsymbol{\alpha})\left(\mathbf{A}_{\omega i} \boldsymbol{\Phi}_{\omega i}^{-1}\right)^{1 / 2}\right] \\
& \frac{\partial\left[\boldsymbol{\Pi}_{\omega i} \boldsymbol{\Sigma}_{\omega i} \boldsymbol{\Pi}_{\omega i}\right]}{\partial \boldsymbol{\omega}_{i}^{\top}}=\boldsymbol{\Pi}_{\omega i} \boldsymbol{\Sigma}_{\omega i} \frac{\partial \boldsymbol{\Pi}_{\omega i}}{\partial \boldsymbol{\omega}_{i}^{\top}}+\boldsymbol{\Pi}_{\omega i} \frac{\partial \boldsymbol{\Sigma}_{\omega i}}{\partial \boldsymbol{\omega}_{i}^{\top}} \boldsymbol{\Pi}_{\omega i}+\frac{\partial \boldsymbol{\Pi}_{\omega i}}{\partial \boldsymbol{\omega}_{i}^{\top}} \boldsymbol{\Sigma}_{\omega i} \boldsymbol{\Pi}_{\omega i}, \\
& \frac{\partial \boldsymbol{\Pi}_{\omega i}}{\partial \boldsymbol{\omega}_{i}^{\top}}=\beta_{k} s_{x_{k}} \dot{\mathbf{G}}_{\omega i} \frac{\partial \boldsymbol{\Pi}_{\omega i}}{\partial \boldsymbol{\mu}_{\omega i}^{\top}}
\end{aligned}
$$

$\dot{\mathbf{G}}_{\omega i}=\operatorname{diag}\left(\partial^{2} g^{-1}\left(\eta_{\omega i 1}\right) / \partial \eta_{\omega i 1}^{2}, \ldots, \partial^{2} g^{-1}\left(\eta_{\omega i t}\right) / \partial \eta_{\omega i t}^{2}\right), \quad \dot{\mathbf{F}}_{\omega i}=\operatorname{diag}\left(\partial^{2} f^{-1}\left(\delta_{\omega i 1}\right) / \partial \delta_{\omega i 1}^{2}\right.$, $\left.\ldots, \partial^{2} f^{-1}\left(\delta_{\omega i t}\right) / \partial \delta_{\omega i t}^{2}\right), \quad \dot{\mathrm{E}}\left(\ddot{\mathbf{c}}_{\omega i}\right)=\operatorname{diag}\left(\partial \mathrm{E}\left(\ddot{\mathrm{c}}_{\omega i 1}\right) / \partial \phi_{\omega i 1}^{-1}, \ldots, \partial \mathrm{E}\left(\ddot{\mathrm{c}}_{\omega i t}\right) / \partial \phi_{\omega i t}^{-1}\right), \quad \mathcal{A}_{\omega i}=$ $\left(\boldsymbol{\Phi}_{\omega i}^{-1} \mathbf{A}_{\omega i}\right)^{1 / 2}\left[\beta_{k} s_{x_{k}} \mathbf{G}_{\omega i} \dot{\boldsymbol{\Upsilon}}_{\omega i} \boldsymbol{\Phi}_{\omega i}^{-1}+\gamma_{k} s_{x_{k}} \mathbf{F}_{\omega i} \mathbf{A}_{\omega i}\right], \quad \partial \boldsymbol{\Pi}_{\omega i} / \partial \boldsymbol{\mu}_{\omega i}^{\top}=\operatorname{diag}\left(\partial \varphi_{\omega i 1} / \partial \mu_{\omega i 1}, \ldots\right.$, $\left.\partial \varphi_{\omega i t} / \partial \mu_{\omega i t}\right)$ e $\mathcal{B}_{2 \omega i}=\operatorname{diag}\left(\mathcal{B}_{2 \omega i 1}, \ldots, \mathcal{B}_{2 \omega i t}\right)$, em que $\dot{\boldsymbol{\Upsilon}}_{\omega i}=\operatorname{diag}\left(\partial v\left(\mu_{\omega i 1}\right) / \partial \mu_{\omega i 1}, \ldots\right.$, $\left.\partial v\left(\mu_{\omega i t}\right) / \partial \mu_{\omega i t}\right)$ e $\mathcal{B}_{2 \omega i j}=\mathrm{y}_{i j} \partial \varphi_{\omega i j} / \partial \mu_{\omega i j}-\left[\left(\partial b\left(\varphi_{\omega i j}\right) / \partial \varphi_{\omega i j}\right)\left(\partial \varphi_{\omega i j} / \partial \mu_{\omega i j}\right)\right]$, com $i=$ $1, \ldots, n$ e $j=1, \ldots, t$. Ressaltamos que as derivadas envolvendo $\varphi_{\omega i j}, \dot{c}_{\omega i j}$ e $\ddot{c}_{\omega i j}$ dependem da distribuição adotada para $\mathrm{y}_{i j}$, cuja densidade deve satisfazer (4.6). A Tabela C.4 apresenta essas derivadas para algumas distribuições pertencentes à família exponencial. 


\subsubsection{A $k^{\prime}$-ésima coluna da matriz $\mathrm{Q}$ igual à $k$-ésima coluna da matriz $\mathrm{X}$}

Considerando que a matriz de covariáveis $\mathbf{Q}$ que modela a precisão tem a $k^{\prime}$-ésima coluna igual à $k$-ésima coluna da matriz $\mathbf{X}$, a qual é perturbada segundo o esquema definido em (6.20), a matriz de covariáveis M modificada é dada por

$$
\mathbf{M}_{\omega}^{\top}=\left(\begin{array}{cc}
\mathbf{X}_{\omega}^{\top} & \mathbf{0} \\
\mathbf{0} & \mathbf{Q}_{\omega}^{\top}
\end{array}\right),
$$

em que o índice $\omega$ indica que as matrizes $\mathbf{X}$ e $\mathbf{Q}$ dependem da perturbação definida em (6.20). Logo, temos

$$
\frac{\partial \mathbf{M}_{\omega}^{\top}}{\partial \boldsymbol{\omega}^{\top}}=\left(\begin{array}{cc}
\frac{\partial \mathbf{X}_{\omega}^{\top}}{\partial \boldsymbol{\omega}^{\top}} & \mathbf{0} \\
\mathbf{0} & \frac{\partial \mathbf{Q}_{\omega}^{\top}}{\partial \boldsymbol{\omega}^{\top}}
\end{array}\right),
$$

sendo uma matriz $(p+q) \times 2 N$, em que a derivada de $\mathbf{X}_{\omega}^{\top}$ com relação à $\boldsymbol{\omega}^{\top}$ é uma matriz $p \times N$ de zeros exceto a $k$-ésima linha que é composta pela constante $s_{x_{k}}$ e a derivada de $\mathbf{Q}_{\omega}^{\top}$ com relação à $\boldsymbol{\omega}^{\top}$ é uma matriz $q \times N$ de zeros exceto a $k^{\prime}$-ésima linha que é composta pela constante $s_{x_{k}}$, com $N=n t$.

As derivadas de $\boldsymbol{\Lambda}_{\omega}, \boldsymbol{\Omega}_{\omega}$ e $\mathbf{b}_{\omega}$ todas com relação à $\boldsymbol{\omega}^{\top}$, quando consideramos cada uma das três equações de estimação: (2.13), (3.12) e (4.10), são iguais às apresentadas na Seção 6.4.3.2 exceto o parâmetro $\gamma_{k}$ que deve ser substituido por $\gamma_{k^{\prime}}$. A seguir, apresentamos essas derivadas para cada uma das três equações de estimação.

BETA

De acordo com a equação de estimação definida em (2.13), as matrizes $\boldsymbol{\Lambda}_{\omega i}$ e $\boldsymbol{\Omega}_{\omega i}$ e o vetor $\mathbf{b}_{\omega i}$ são dados na equação (6.49), mas sendo a matriz $\mathbf{M}_{\omega}$ dada em (6.57). Assim, da Seção 6.4.3.2, obtemos

$$
\begin{aligned}
\frac{\partial \boldsymbol{\Lambda}_{\omega i}}{\partial \boldsymbol{\omega}_{i}^{\top}} & =\left(\begin{array}{c}
\beta_{k} s_{x_{k}} \boldsymbol{\Phi}_{\omega i}\left[\dot{\mathbf{G}}_{\omega i} \mathbf{A}_{\omega i}+\mathbf{G}_{\omega i}^{2} \mathcal{E}_{1 \omega i}\right]+\gamma_{k^{\prime}} s_{x_{k}} \mathbf{G}_{\omega i} \mathbf{F}_{\omega i}\left[\mathbf{A}_{\omega i}+\mathbf{\Phi}_{\omega i} \mathcal{E}_{2 \omega i}\right] \\
\beta_{k} s_{x_{k}} \mathbf{F}_{\omega i} \mathbf{C}_{\omega i}\left[\mathbf{A}_{\omega i}+\mathcal{M}_{\omega i} \mathcal{E}_{1 \omega i}\right]+\gamma_{k^{\prime}} s_{x_{k}}\left[\dot{\mathbf{F}}_{\omega i} \mathbf{C}_{\omega i}-\mathbf{F}_{\omega i}^{2}\left(\mathbf{I}_{t}-\mathcal{M}_{\omega i}\right) \mathcal{E}_{2 \omega i}\right]
\end{array}\right), \\
\frac{\partial \boldsymbol{\Omega}_{\omega i}}{\partial \boldsymbol{\omega}_{i}^{\top}}=\frac{1}{2}\left[\mathbf{A}_{\omega i}^{1 / 2} \mathbf{R}(\boldsymbol{\alpha}) \mathcal{A}_{\omega i}+\mathcal{A}_{\omega i} \mathbf{R}(\boldsymbol{\alpha}) \mathbf{A}_{\omega i}^{1 / 2}\right] \mathrm{e} & \frac{\partial \mathbf{b}_{\omega i}}{\partial \boldsymbol{\omega}_{i}^{\top}}=-\left[\beta_{k} s_{x_{k}} \mathbf{G}_{\omega i} \boldsymbol{\Phi}_{\omega i} \mathbf{A}_{\omega i}+\gamma_{k^{\prime}} s_{x_{k}} \mathbf{F}_{\omega i} \mathcal{E}_{3 \omega i}\right],
\end{aligned}
$$

em que $\mathcal{A}_{\omega i}=\mathbf{A}_{\omega i}^{-1 / 2}\left[\beta_{k} s_{x_{k}} \mathbf{G}_{\omega i} \mathcal{E}_{1 \omega i}+\gamma_{k^{\prime}} s_{x_{k}} \mathbf{F}_{\omega i} \mathcal{E}_{2 \omega i}\right]$ e $\mathcal{E}_{1 \omega i}, \mathcal{E}_{2 \omega i}, \mathcal{E}_{3 \omega i}, \mathcal{M}_{\omega i}, \dot{\mathbf{G}}_{\omega i}$ e $\dot{\mathbf{F}}_{\omega i}$ estão descritos na Seção 6.4.3.2 para o caso BETA, com $i=1, \ldots, n$ e $j=1, \ldots, t$. 


\section{Simplex}

De acordo com a equação de estimação definida em (3.12), as matrizes $\boldsymbol{\Lambda}_{\omega i}$ e $\boldsymbol{\Omega}_{\omega i}$ e o vetor $\mathbf{b}_{\omega i}$ são dados na equação (6.53), entretanto, envolvendo a matriz $\mathbf{M}_{\omega}$ conforme equação (6.57). Assim, da Seção 6.4.3.2, obtemos

$$
\begin{aligned}
\frac{\partial \boldsymbol{\Lambda}_{\omega i}}{\partial \boldsymbol{\omega}_{i}^{\top}} & =\left(\begin{array}{cc}
\beta_{k} s_{x_{k}} \boldsymbol{\Phi}_{\omega i}\left[\dot{\mathbf{G}}_{\omega i} \mathbf{A}_{\omega i}+\mathbf{G}_{\omega i}^{2} \mathcal{E}_{1 \omega i}\right]-\gamma_{k^{\prime}} s_{x_{k}} \mathbf{G}_{\omega i} \mathbf{F}_{\omega i} \boldsymbol{\Phi}_{\omega i}^{2}\left[\mathbf{A}_{\omega i}+\mathbf{\Phi}_{\omega i} \mathcal{E}_{2 \omega i}\right] & \mathbf{0} \\
\mathbf{0} & \gamma_{k^{\prime}} s_{x_{k}} \dot{\mathbf{F}}_{\omega i}
\end{array}\right), \\
\frac{\partial \boldsymbol{\Omega}_{\omega i}}{\partial \boldsymbol{\omega}_{i}^{\top}} & =\left(\begin{array}{cc}
\frac{1}{2}\left[\mathbf{A}_{\omega i}^{1 / 2} \mathbf{R}(\boldsymbol{\alpha}) \mathcal{A}_{\omega i}+\mathcal{A}_{\omega i} \mathbf{R}(\boldsymbol{\alpha}) \mathbf{A}_{\omega i}^{1 / 2}\right] & \mathbf{0} \\
\mathbf{0} & 4 \gamma_{k^{\prime}} s_{x_{k}} \mathbf{F}_{\omega i} \boldsymbol{\Phi}_{\omega i}^{-1}
\end{array}\right) \\
\frac{\partial \mathbf{b}_{\omega i}}{\partial \boldsymbol{\omega}_{i}^{\top}} & =\left(\begin{array}{c}
\beta_{k} s_{x_{k}} \mathbf{G}_{\omega i} \mathcal{U}_{\omega i} \\
-\left[2 \beta_{k} s_{x_{k}} \mathbf{G}_{\omega i} \operatorname{diag}\left(\mathbf{u}_{\omega i}\right)+\gamma_{k^{\prime}} s_{x_{k}} \mathbf{F}_{\omega i}\right]
\end{array}\right),
\end{aligned}
$$

sendo $\mathcal{A}_{\omega i}=\mathbf{A}_{\omega i}^{-1 / 2}\left[\beta_{k} s_{x_{k}} \mathbf{G}_{\omega i} \mathcal{E}_{1 \omega i}-\gamma_{k^{\prime}} s_{x_{k}} \mathbf{F}_{\omega i} \boldsymbol{\Phi}_{\omega i}^{2} \mathcal{E}_{2 \omega i}\right]$ e $\mathcal{E}_{1 \omega i}, \mathcal{E}_{2 \omega i}, \quad \dot{\mathbf{G}}_{\omega i}$ e $\dot{\mathbf{F}}_{\omega i}$ estão descritos na Seção 6.4.3.2 para o caso Simplex, com $i=1, \ldots, n$ e $j=1, \ldots, t$.

\section{Modelos Lineares Generalizados}

De acordo com a equação de estimação definida em (4.10), as matrizes $\boldsymbol{\Lambda}_{\omega i}$ e $\boldsymbol{\Omega}_{\omega i}$ e o vetor $\mathbf{b}_{\omega i}$ são dados na equação (6.56), mas considerando a matriz $\mathbf{M}_{\omega}$ dada na equação (6.57). Assim, da Seção 6.4.3.2, obtemos

$$
\begin{aligned}
\frac{\partial \boldsymbol{\Lambda}_{\omega i}}{\partial \boldsymbol{\omega}_{i}^{\top}} & =\left(\begin{array}{cc}
\beta_{k} s_{x_{k}} \dot{\mathbf{G}}_{\omega i} & \mathbf{0} \\
\mathbf{0} & \gamma_{k^{\prime}} s_{x_{k}}\left[\dot{\mathbf{F}}_{\omega i} \mathrm{E}\left(\ddot{\mathbf{c}}_{\omega i}\right)+\mathbf{F}_{\omega i}^{2} \dot{\mathrm{E}}\left(\ddot{\mathbf{c}}_{\omega i}\right)\right]
\end{array}\right), \\
\frac{\partial \boldsymbol{\Omega}_{\omega i}}{\partial \boldsymbol{\omega}_{i}^{\top}} & =\left(\begin{array}{cc}
\frac{\partial \boldsymbol{\Sigma}_{\omega i}}{\partial \boldsymbol{\omega}_{i}^{\top}} & \mathbf{0} \\
\mathbf{0} & \frac{\partial\left[\boldsymbol{\Pi}_{\omega i} \boldsymbol{\Sigma}_{\omega i} \boldsymbol{\Pi}_{\omega i}\right]}{\partial \boldsymbol{\omega}_{i}^{\top}}
\end{array}\right) \mathrm{e} \\
\frac{\partial \mathbf{b}_{\omega i}}{\partial \boldsymbol{\omega}_{i}^{\top}} & =\left(\begin{array}{c}
\beta_{k} s_{x_{k}} \mathbf{G}_{\omega i} \\
\beta_{k} s_{x_{k}} \mathbf{G}_{\omega i} \mathcal{B}_{2 \omega i}+\gamma_{k^{\prime}} s_{x_{k}} \mathbf{F}_{\omega i} \ddot{\mathbf{c}}_{\omega i}
\end{array}\right),
\end{aligned}
$$

sendo $\mathcal{A}_{\omega i}=\left(\boldsymbol{\Phi}_{\omega i}^{-1} \mathbf{A}_{\omega i}\right)^{1 / 2}\left[\beta_{k} s_{x_{k}} \mathbf{G}_{\omega i} \dot{\boldsymbol{\Upsilon}}_{\omega i} \boldsymbol{\Phi}_{\omega i}^{-1}+\gamma_{k^{\prime}} s_{x_{k}} \mathbf{F}_{\omega i} \mathbf{A}_{\omega i}\right]$ e $\dot{\mathbf{G}}_{\omega i}, \quad \dot{\mathbf{F}}_{\omega i}, \quad \dot{\mathrm{E}}\left(\ddot{\mathbf{c}}_{\omega i}\right)$ e $\mathcal{B}_{2 \omega i}$ estão descritos na Seção 6.4.3.2 para o caso Modelos Lineares Generalizados, com $i=1, \ldots, n$ e $j=1, \ldots, t$.

\subsubsection{A $k^{\prime}$-ésima coluna da matriz Q funcão da $k$-ésima coluna da matriz X}

Considere que a matriz de covariáveis $\mathbf{Q}$ que modela a precisão tem a $k^{\prime}$-ésima coluna função da $k$-ésima coluna da matriz $\mathbf{X}$, ou seja, $\mathrm{q}_{\omega i j k^{\prime}}=h\left(\mathrm{x}_{\omega i j k}\right), \operatorname{com} i=1, \ldots, n$, $j=1, \ldots, t, k=2, \ldots, p$ e $k^{\prime}=2, \ldots, q$, em que $h$ é uma função contínua e diferenciável. 
Assim, a matriz de covariáveis $\mathbf{M}$ modificada segundo o esquema definido em (6.20) pode ser expressa como a equação (6.57). Logo, temos

$$
\frac{\partial \mathbf{M}_{\omega}^{\top}}{\partial \boldsymbol{\omega}^{\top}}=\left(\begin{array}{cc}
\frac{\partial \mathbf{X}_{\omega}^{\top}}{\partial \boldsymbol{\omega}^{\top}} & \mathbf{0} \\
\mathbf{0} & \frac{\partial \mathbf{Q}_{\omega}^{\top}}{\partial \boldsymbol{\omega}^{\top}}
\end{array}\right),
$$

sendo uma matriz $(p+q) \times 2 N$, em que a derivada de $\mathbf{X}_{\omega}^{\top}$ com relação à $\boldsymbol{\omega}^{\top}$ é uma matriz $p \times N$ de zeros exceto a $k$-ésima linha que é composta pela constante $s_{x_{k}}$ e a derivada de $\mathbf{Q}_{\omega}^{\top}$ com relação à $\boldsymbol{\omega}^{\top}$ é uma matriz $q \times N$ de zeros exceto a $k^{\prime}$-ésima linha que é composta pelo vetor $\dot{h}\left(\mathrm{x}_{\omega k}\right) s_{x_{k}}$, em que $N=n t$ e $\dot{h}\left(\mathbf{x}_{\omega k}\right)=\left(\dot{h}\left(\mathrm{x}_{\omega 11 k}\right), \dot{h}\left(\mathrm{x}_{\omega 12 k}\right), \ldots, \dot{h}\left(\mathrm{x}_{\omega n t k}\right)\right)$, $\operatorname{com} \dot{h}\left(\mathrm{x}_{\omega i j k}\right)=\partial h\left(\mathrm{x}_{\omega i j k}\right) / \partial \mathrm{x}_{\omega i j k}, \operatorname{com} i=1, \ldots, n, j=1, \ldots, t$ e $k=2, \ldots, p$.

As derivadas de $\boldsymbol{\Lambda}_{\omega}, \boldsymbol{\Omega}_{\omega}$ e $\mathbf{b}_{\omega}$ todas com relação à $\boldsymbol{\omega}^{\top}$, quando consideramos cada das equações de estimação: (2.13), (3.12) e (4.10), são iguais às apresentadas na Seção 6.4.3.2 exceto o parâmetro $\gamma_{k}$ que deve ser substituido por $\gamma_{k^{\prime}} \dot{\mathcal{H}}_{\omega i}$, em que $\dot{\mathcal{H}}_{\omega i}=\operatorname{diag}\left(\dot{h}\left(\mathbf{x}_{\omega k}\right)\right)$. A seguir, apresentamos essas derivadas para cada uma das três equações de estimação.

\section{BETA}

De acordo com a equação de estimação definida em (2.13), as matrizes $\boldsymbol{\Lambda}_{\omega i}$ e $\boldsymbol{\Omega}_{\omega i}$ e o vetor $\mathbf{b}_{\omega i}$ são dados na equação (6.49), sendo $\mathbf{M}_{\omega}$ conforme definida nesta seção. Assim, da Seção 6.4.3.2, obtemos

$$
\begin{aligned}
& \frac{\partial \boldsymbol{\Lambda}_{\omega i}}{\partial \boldsymbol{\omega}_{i}^{\top}}=\left(\begin{array}{c}
\beta_{k} s_{x_{k}} \boldsymbol{\Phi}_{\omega i}\left[\dot{\mathbf{G}}_{\omega i} \mathbf{A}_{\omega i}+\mathbf{G}_{\omega i}^{2} \mathcal{E}_{1 \omega i}\right]+\gamma_{k^{\prime}} s_{x_{k}} \dot{\mathcal{H}}_{\omega i} \mathbf{G}_{\omega i} \mathbf{F}_{\omega i}\left[\mathbf{A}_{\omega i}+\mathbf{\Phi}_{\omega i} \mathcal{E}_{2 \omega i}\right] \\
\beta_{k} s_{x_{k}} \mathbf{F}_{\omega i} \mathbf{C}_{\omega i}\left[\mathbf{A}_{\omega i}+\mathcal{M}_{\omega i} \mathcal{E}_{1 \omega i}\right]+\gamma_{k^{\prime}} s_{x_{k}} \dot{\mathcal{H}}_{\omega i}\left[\dot{\mathbf{F}}_{\omega i} \mathbf{C}_{\omega i}-\mathbf{F}_{\omega i}^{2}\left(\mathbf{I}_{t}-\mathcal{M}_{\omega i}\right) \mathcal{E}_{2 \omega i}\right]
\end{array}\right), \\
& \frac{\partial \boldsymbol{\Omega}_{\omega i}}{\partial \boldsymbol{\omega}_{i}^{\top}}=\frac{1}{2}\left[\mathbf{A}_{\omega i}^{1 / 2} \mathbf{R}(\boldsymbol{\alpha}) \mathcal{A}_{\omega i}+\mathcal{A}_{\omega i} \mathbf{R}(\boldsymbol{\alpha}) \mathbf{A}_{\omega i}^{1 / 2}\right] \mathrm{e} \\
& \frac{\partial \mathbf{b}_{\omega i}}{\partial \boldsymbol{\omega}_{i}^{\top}}=-\left[\beta_{k} s_{x_{k}} \mathbf{G}_{\omega i} \boldsymbol{\Phi}_{\omega i} \mathbf{A}_{\omega i}+\gamma_{k^{\prime}} s_{x_{k}} \dot{\mathcal{H}}_{\omega i} \mathbf{F}_{\omega i} \mathcal{E}_{3 \omega i}\right]
\end{aligned}
$$

em que $\mathcal{A}_{\omega i}=\mathbf{A}_{\omega i}^{-1 / 2}\left[\beta_{k} s_{x_{k}} \mathbf{G}_{\omega i} \mathcal{E}_{1 \omega i}+\gamma_{k^{\prime}} s_{x_{k}} \dot{\mathcal{H}}_{\omega i} \mathbf{F}_{\omega i} \mathcal{E}_{2 \omega i}\right]$ e $\mathcal{E}_{1 \omega i}, \mathcal{E}_{2 \omega i}, \mathcal{E}_{3 \omega i}, \mathcal{M}_{\omega i}, \dot{\mathbf{G}}_{\omega i}$ e $\dot{\mathbf{F}}_{\omega i}$ estão descritos na Seção 6.4.3.2 para o caso BETA, com $i=1, \ldots, n$ e $j=1, \ldots, t$.

\section{Simplex}

De acordo com a equação de estimação definida em (3.12), as matrizes $\boldsymbol{\Lambda}_{\omega i}$ e $\boldsymbol{\Omega}_{\omega i}$ e o vetor $\mathbf{b}_{\omega i}$ são dados na equação (6.53), sendo $\mathbf{M}_{\omega}$ conforme definida nesta seção. Assim, da Seção 6.4.3.2, obtemos 


$$
\begin{aligned}
& \frac{\partial \boldsymbol{\Lambda}_{\omega i}}{\partial \boldsymbol{\omega}_{i}^{\top}}=\operatorname{diag}\left(\beta_{k} s_{x_{k}} \boldsymbol{\Phi}_{\omega i}\left[\dot{\mathbf{G}}_{\omega i} \mathbf{A}_{\omega i}+\mathbf{G}_{\omega i}^{2} \mathcal{E}_{1 \omega i}\right]-\gamma_{k^{\prime}} s_{x_{k}} \dot{\mathcal{H}}_{\omega i} \mathbf{G}_{\omega i} \mathbf{F}_{\omega i} \boldsymbol{\Phi}_{\omega i}^{2}\left[\mathbf{A}_{\omega i}+\boldsymbol{\Phi}_{\omega i} \mathcal{E}_{2 \omega i}\right]\right. \\
& \left.\gamma_{k^{\prime}} s_{x_{k}} \dot{\mathcal{H}}_{\omega i} \dot{\mathbf{F}}_{\omega i}\right) \\
& \frac{\partial \boldsymbol{\Omega}_{\omega i}}{\partial \boldsymbol{\omega}_{i}^{\top}}=\left(\begin{array}{cc}
\frac{1}{2}\left[\mathbf{A}_{\omega i}^{1 / 2} \mathbf{R}(\boldsymbol{\alpha}) \mathcal{A}_{\omega i}+\mathcal{A}_{\omega i} \mathbf{R}(\boldsymbol{\alpha}) \mathbf{A}_{\omega i}^{1 / 2}\right] & \mathbf{0} \\
\mathbf{0} & 4 \gamma_{k^{\prime}} s_{x_{k}} \dot{\mathcal{H}}_{\omega i} \mathbf{F}_{\omega i} \boldsymbol{\Phi}_{\omega i}^{-1}
\end{array}\right) \quad \mathrm{e} \\
& \frac{\partial \mathbf{b}_{\omega i}}{\partial \boldsymbol{\omega}_{i}^{\top}}=\left(\begin{array}{c}
\beta_{k} s_{x_{k}} \mathbf{G}_{\omega i} \mathcal{U}_{\omega i} \\
-\left[2 \beta_{k} s_{x_{k}} \mathbf{G}_{\omega i} \operatorname{diag}\left(\mathbf{u}_{\omega i}\right)+\gamma_{k^{\prime}} s_{x_{k}} \dot{\mathcal{H}}_{\omega i} \mathbf{F}_{\omega i}\right]
\end{array}\right)
\end{aligned}
$$

sendo $\mathcal{A}_{\omega i}=\mathbf{A}_{\omega i}^{-1 / 2}\left[\beta_{k} s_{x_{k}} \mathbf{G}_{\omega i} \mathcal{E}_{1 \omega i}-\gamma_{k^{\prime}} s_{x_{k}} \dot{\mathcal{H}}_{\omega i} \mathbf{F}_{\omega i} \boldsymbol{\Phi}_{\omega i}^{2} \mathcal{E}_{2 \omega i}\right]$ e $\mathcal{E}_{1 \omega i}, \mathcal{E}_{2 \omega i}, \dot{\mathbf{G}}_{\omega i}$ e $\dot{\mathbf{F}}_{\omega i}$ estão descritos na Seção 6.4.3.2 para o caso Simplex, com $i=1, \ldots, n$ e $j=1, \ldots, t$.

\section{Modelos Lineares Generalizados}

De acordo com a equação de estimação definida em (4.10), as matrizes $\boldsymbol{\Lambda}_{\omega i}$ e $\boldsymbol{\Omega}_{\omega i}$ e o vetor $\mathbf{b}_{\omega i}$ são dados na equação (6.56), sendo $\mathbf{M}_{\omega}$ conforme definida nesta seção. Assim, da Seção 6.4.3.2, obtemos

$$
\begin{aligned}
\frac{\partial \boldsymbol{\Lambda}_{\omega i}}{\partial \boldsymbol{\omega}_{i}^{\top}} & =\left(\begin{array}{cc}
\beta_{k} s_{x_{k}} \dot{\mathbf{G}}_{\omega i} & \mathbf{0} \\
\mathbf{0} & \gamma_{k^{\prime}} s_{x_{k}} \dot{\mathcal{H}}_{\omega i}\left[\dot{\mathbf{F}}_{\omega i} \mathrm{E}\left(\ddot{\mathbf{c}}_{\omega i}\right)+\mathbf{F}_{\omega i}^{2} \dot{\mathrm{E}}\left(\ddot{\mathbf{c}}_{\omega i}\right)\right]
\end{array}\right), \\
\frac{\partial \boldsymbol{\Omega}_{\omega i}}{\partial \boldsymbol{\omega}_{i}^{\top}}= & \left(\begin{array}{cc}
\frac{\partial \boldsymbol{\Sigma}_{\omega i}}{\partial \boldsymbol{\omega}_{i}^{\top}} & \mathbf{0} \\
\mathbf{0} & \frac{\partial\left[\boldsymbol{\Pi}_{\omega i} \boldsymbol{\Sigma}_{\omega i} \boldsymbol{\Pi}_{\omega i}\right]}{\partial \boldsymbol{\omega}_{i}^{\top}}
\end{array}\right) \quad \mathrm{e} \\
\frac{\partial \mathbf{b}_{\omega i}}{\partial \boldsymbol{\omega}_{i}^{\top}}= & \left.\begin{array}{c}
-\beta_{k} s_{x_{k}} \mathbf{G}_{\omega i} \\
\beta_{k} s_{x_{k}} \mathbf{G}_{\omega i} \mathcal{B}_{2 \omega i}+\gamma_{k^{\prime}} s_{x_{k}} \dot{\mathcal{H}}_{\omega i} \mathbf{F}_{\omega i} \ddot{\mathbf{c}}_{\omega i}
\end{array}\right),
\end{aligned}
$$

sendo $\mathcal{A}_{\omega i}=\left(\boldsymbol{\Phi}_{\omega i}^{-1} \mathbf{A}_{\omega i}\right)^{1 / 2}\left[\beta_{k} s_{x_{k}} \mathbf{G}_{\omega i} \dot{\boldsymbol{\Upsilon}}_{\omega i} \boldsymbol{\Phi}_{\omega i}^{-1}+\gamma_{k^{\prime}} s_{x_{k}} \dot{\mathcal{H}}_{\omega i} \mathbf{F}_{\omega i} \mathbf{A}_{\omega i}\right]$ e $\dot{\mathbf{G}}_{\omega i}, \dot{\mathbf{F}}_{\omega i}, \dot{\mathrm{E}}\left(\ddot{\mathbf{c}}_{\omega i}\right)$ e $\mathcal{B}_{2 \omega i}$ estão descritos na Seção 6.4.3.2 para o caso Modelos Lineares Generalizados, com $i=1, \ldots, n$ e $j=1, \ldots, t$. 


\section{Parte III}

\section{Simulações e Aplicações}




\section{Capítulo 7}

\section{Aplicações a dados simulados}

Considerando os cenários em que há baixa e alta correlação entre as observações da mesma unidade experimental, neste capítulo apresentamos algumas aplicações a dados simulados para avaliar o impacto que a escolha de uma estrutura de correlação de trabalho causa nas medidas de influência local quando essa difere ou não da verdadeira matriz de correlação. Além disso, avaliamos o comportamento das medidas de influência local com esquema de perturbação ponderação de casos quando os dados são gerados com dispersão variável, mas ajustados com um modelo sob homogeneidade da dispersão.

A seguir, avaliamos esses casos gerando dados com distribuições normais multivariadas e dados com distribuições marginais de Poisson correlacionadas, utilizando as equações de estimação apresentadas no Capítulo 4.

\subsection{Simulação I - Dados com distribuição Normal}

Inicialmente, simulamos quatro bancos de dados com distribuição normal multivariada ${ }^{1}$ com as seguintes estruturas de correlação:

1. Padrão uniforme com baixa correlação $(\alpha=0,3)$;

2. Padrão uniforme com alta correlação $(\alpha=0,9)$;

3. AR-1 com baixa correlação $(\alpha=0,3)$; e

4. AR-1 com alta correlação $(\alpha=0,9)$.

${ }^{1}$ Para gerar dados com distribuição normal multivariada, utilizamos o comando rmvnorm existente na library mvtnorm do software R (http://www.r-project.org/). 
Esses dados foram gerados considerando o modelo de regressão para a média dado por

$$
\mu_{i j}=10-1,5 \mathrm{x}_{i j}
$$

sendo $\mathrm{x}_{i j}$ os valores de uma covariável (denominada tempo) gerados por uma Poisson de média 10 , em que $i=1, \ldots, 50$ e $j=1, \ldots, 5$. Além disso, consideramos homogeneidade do parâmetro de dispersão com $\phi^{-1}$ igual a 0,1 . Lembramos que, na distribuição normal, a função de variância vale um tornando a matriz de covariâncias igual ao parâmetro de dispersão multiplicado pela matriz de correlação.

A Figura 7.1 apresenta gráficos de dispersão das variáveis respostas simuladas versus seus respectivos valores da covariável tempo. As retas ajustadas foram traçadas considerando os verdadeiros valores dos parâmetros de regressão $\left(\beta_{0}=10\right.$ e $\left.\beta_{1}=-1,5\right)$. Nesses gráficos, não notamos pontos gerados com comportamento muito diferente (afastados) dos demais.

Cada banco de dados simulado foi ajustado pelo modelo $\mu_{i j}=\beta_{0}+\beta_{1} \mathrm{x}_{i j}$ sob homogeneidade da dispersão, considerando quatro estruturas diferentes para a matriz de correlação de trabalho: a verdadeira matriz de correlação que gerou os dados e com as estruturas padrão uniforme, auto-regressiva de ordem um (AR-1) e independente.

As Figuras 7.2 a 7.5 apresentam, para esses ajustes, os gráficos de influência local para os esquemas de perturbação ponderação de casos, da variável resposta e da precisão. Ressaltamos que o esquema de perturbação da covariável tempo é inviável por não ser contínua.

Analisando a Figura 7.2, cujos dados foram gerados com baixa correlação e matriz de correlação padrão uniforme, observamos que os pontos que se destacam como mais influentes no ajuste que considera a verdadeira matriz de correlação também se destacam nos gráficos de influência obtidos do ajuste que considera a mesma estrutura que a verdadeira, ou seja, padrão uniforme, mesmo o valor estimado para a correlação sendo um pouco inferior ao verdadeiro valor $(\hat{\alpha}=0,267)$. Nos ajustes em que consideramos estruturas de correlação diferentes da verdadeira, ou seja, AR-1 e independente, ainda notamos os mesmos pontos se destacando nos gráficos de influência local com esquema de perturbação ponderação de casos e da precisão. Além disso, não há pontos se destacando como influentes em nenhum dos gráficos de influência local com perturbação da variável resposta.

Essas interpretações também se estendem para a Figura 7.4, cujos dados foram gerados com baixa correlação e matriz de correlação AR-1. Nesse caso, fazemos conclusões corretas quando utilizamos os gráficos de influência obtidos dos ajustes que consideram a mesma estrutura que a verdadeira (ou seja, AR-1) ou a padrão uniforme, pois os pontos que 
se destacam nesses gráficos são os mesmos quando consideramos, no ajuste, a verdadeira matriz de correlação. Mas, apesar de ser baixa a correlação entre as observações da mesma unidade experimental, não teríamos conclusões tão corretas se assumimos como correlação de trabalho a independência entre as observações da mesma unidade experimetal, pois a unidade experimental 42 avaliada com tempo $5,(42,5)$, não se destaca como um possível ponto influente nos gráficos de ponderação de casos e precisão. Além disso, o gráfico de influência local com perturbação da variável resposta não detecta pontos influentes como acontece no gráfico de influência local com perturbação da variável resposta obtido do ajuste que considera a verdadeira correlação.

Nas Figuras 7.3 e 7.5, cujos dados foram gerados com alta correlação entre as observações da mesma unidade experimental, temos que as conclusões são as mesmas com relação às observações destacadas como possíveis pontos influentes apenas quando o ajuste tem estrutura de correlação de trabalho igual à da verdadeira matriz de correlação que gerou os dados. Modificar essa estrutura pode nos levar a conclusões erradas quanto aos pontos que são influentes nas estimativas dos parâmetros de regressão. Isso fica nítido se, por exemplo na Figura 7.3, notarmos que os possíveis pontos influentes destacados no gráfico de ponderação de casos quando consideramos no ajuste a verdadeira matriz de correlação são as observações $(4,8)$ e $(4,19)$, enquanto que o gráfico de ponderação de casos obtido do ajuste com estrutura AR-1, que é nesse caso diferente da estrutura da verdadeira matriz de correlação que gerou os dados, destaca a unidade experimental 4 com tempo 16, ou seja, o ponto $(4,16)$.

Foram gerados diversos bancos de dados com modelagens iguais a um dos quatro bancos ilustrados anteriormente. De maneira geral, pudemos concluir que, com baixa ou alta correlação entre as observações da mesma unidade experimental, temos os mesmos pontos destacados como influentes quando se usa no ajuste uma estrutura de correlação igual à da verdadeira matriz de correlação que gerou os dados. Quando se considera no ajuste uma estrutura de correlação de trabalho diferente da estrutura da verdadeira matriz de correlação nem sempre se tem os mesmos pontos destacados como influentes, principalmente, se for alta a correlação entre as observações.

Para avaliar o comportamento da medida de influência local com esquema de perturbação ponderação de casos quando há heterogeneidade da dispersão mas os dados são ajustados sob a suposição de homogeneidade do mesmo, simulamos dois bancos de dados alternando a estrutura de correlação padrão uniforme e AR-1. Ambas com alta correlação entre as observações da mesma unidade experimental $(\alpha=0,9)$. 
Verdadeira $\mathbf{R}(\boldsymbol{\alpha})$ igual a Padrão Uniforme

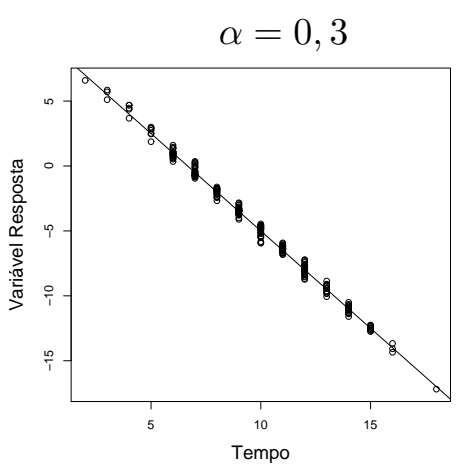

(a)

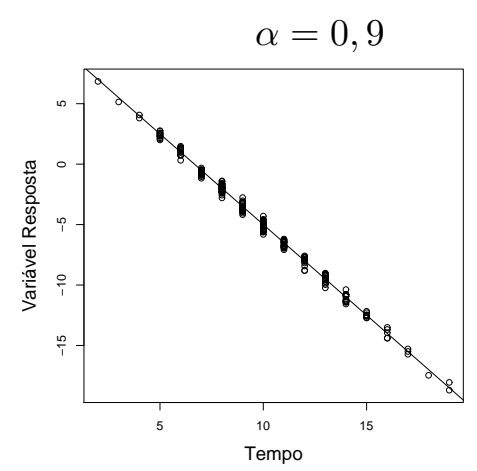

(b)

Verdadeira $\mathbf{R}(\boldsymbol{\alpha})$ igual a AR-1

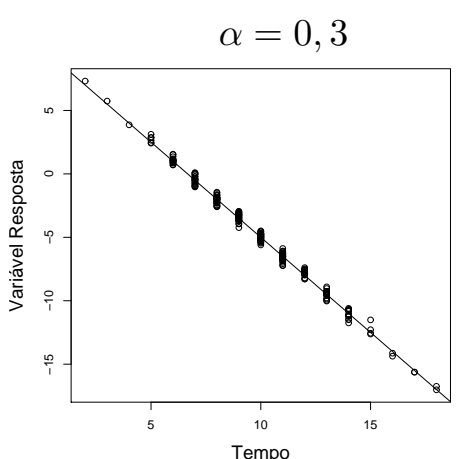

(c)

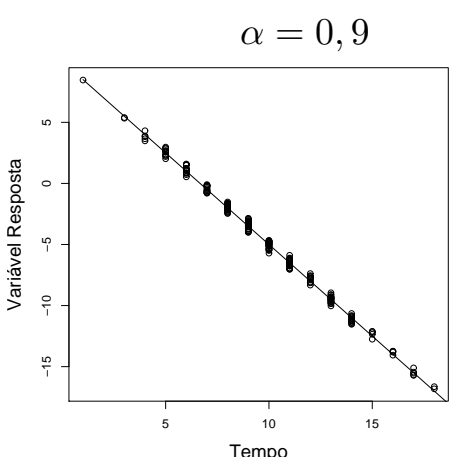

(d)

Figura 7.1: Valores simulados de uma Normal considerando estrutura padrão uniforme com $\alpha=0,3$ (a) e com $\alpha=0,9$ (b) e estrutura AR-1 $\operatorname{com} \alpha=0,3$ (c) e com $\alpha=0,9$ (d). Simulação Normal sob homogeneidade da dispersão. 
Verdadeira $\mathbf{R}(\boldsymbol{\alpha})$ igual a Padrão Uniforme e $\alpha=0,3$
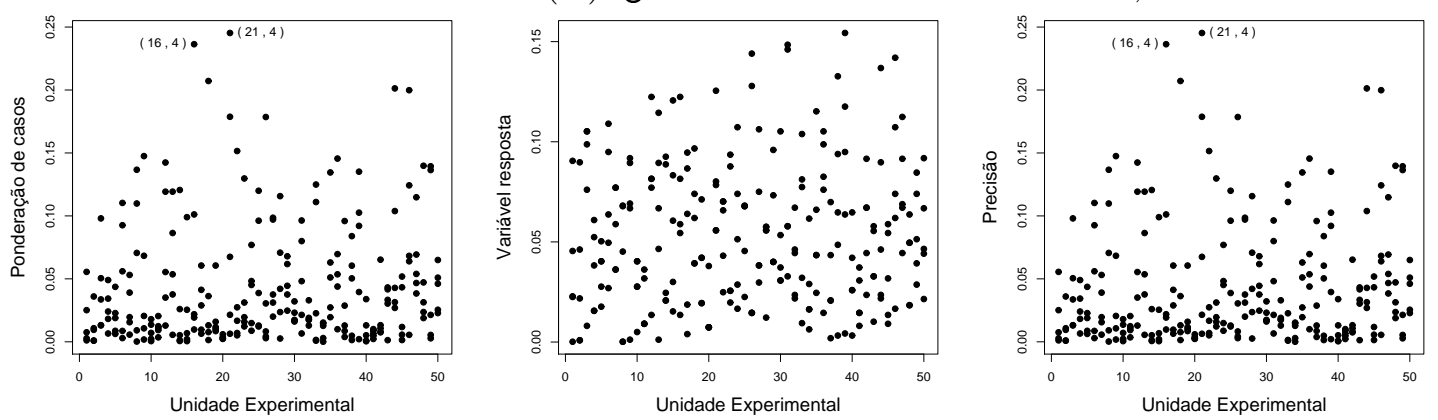

$\mathbf{R}(\boldsymbol{\alpha})$ estimada pela Padrão Uniforme
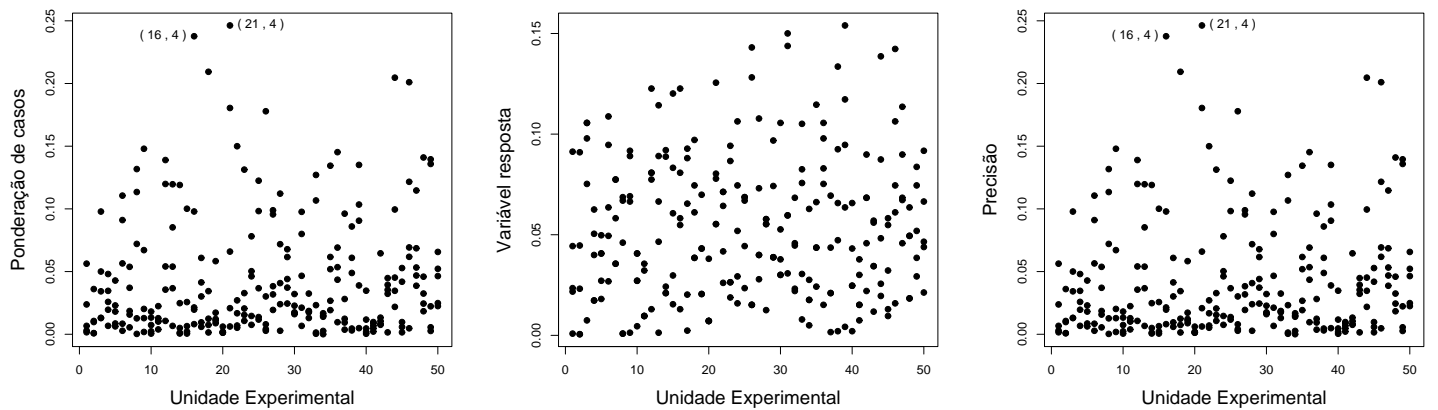

$\mathbf{R}(\boldsymbol{\alpha})$ estimada pela AR-1
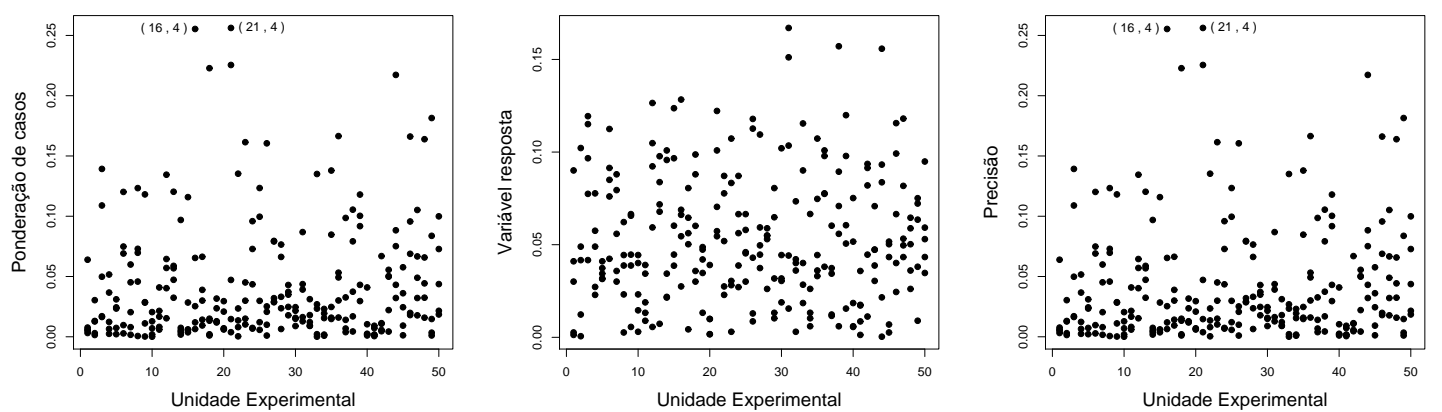

$\mathbf{R}(\boldsymbol{\alpha})$ estimada pela Independente
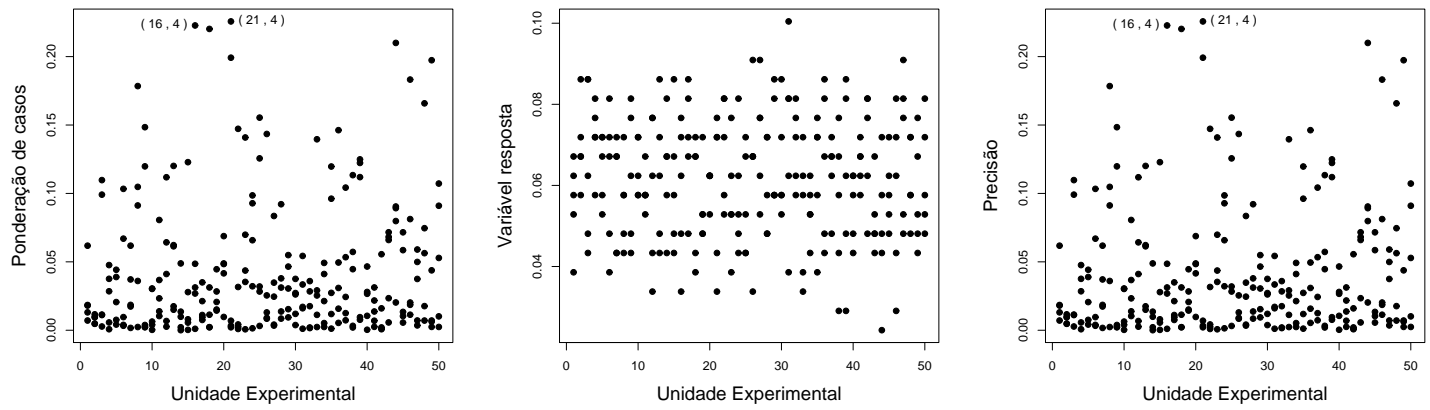

Figura 7.2: Gráficos de influência local considerando a verdadeira matriz de correlação (com estrutura padrão uniforme e $\alpha=0,3$ ) e com matriz de correlação de trabalho padrão uniforme, AR-1 e independente. Simulação Normal sob homogeneidade da dispersão. 
Verdadeira $\mathbf{R}(\boldsymbol{\alpha})$ igual a Padrão Uniforme e $\alpha=0,9$
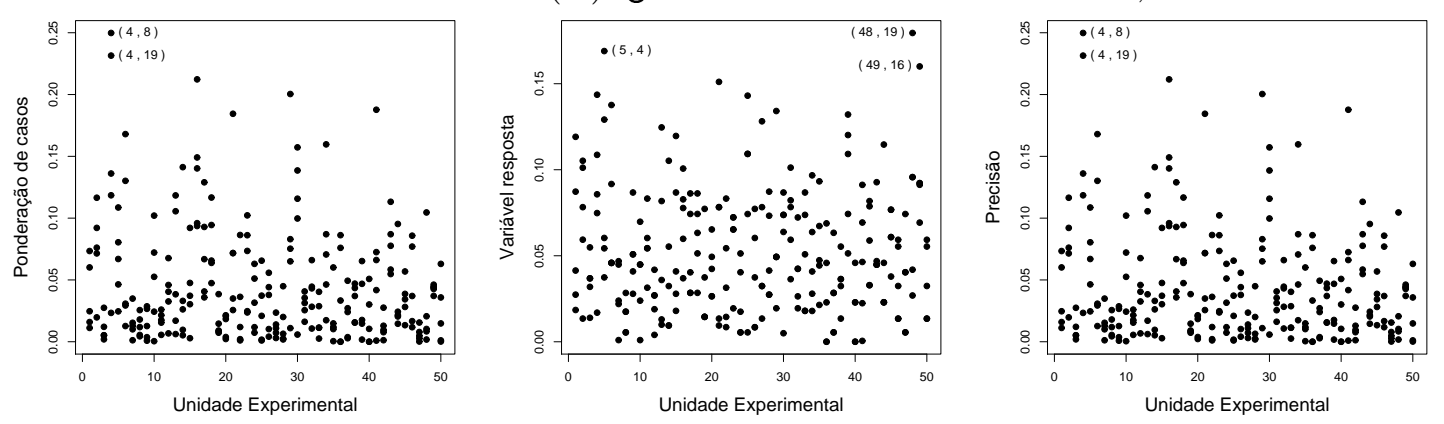

$\mathbf{R}(\boldsymbol{\alpha})$ estimada pela Padrão Uniforme
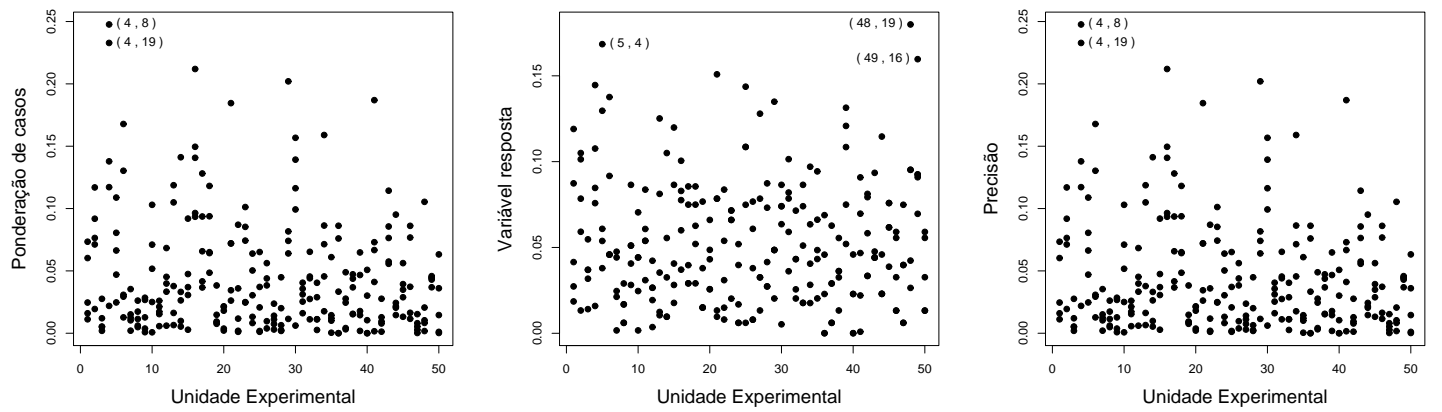

$\mathbf{R}(\boldsymbol{\alpha})$ estimada pela AR-1
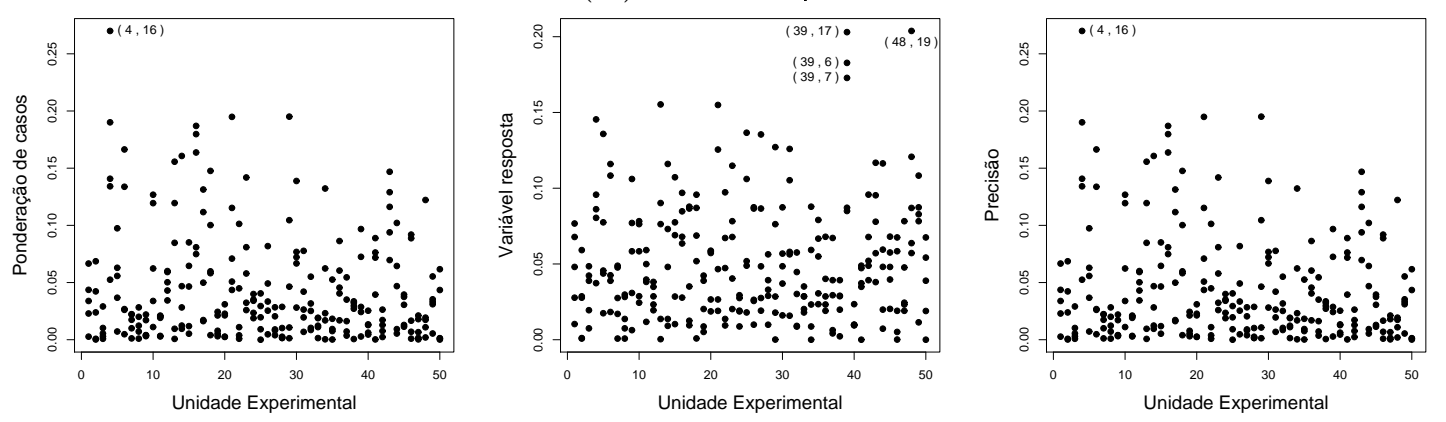

$\mathbf{R}(\boldsymbol{\alpha})$ estimada pela Independente
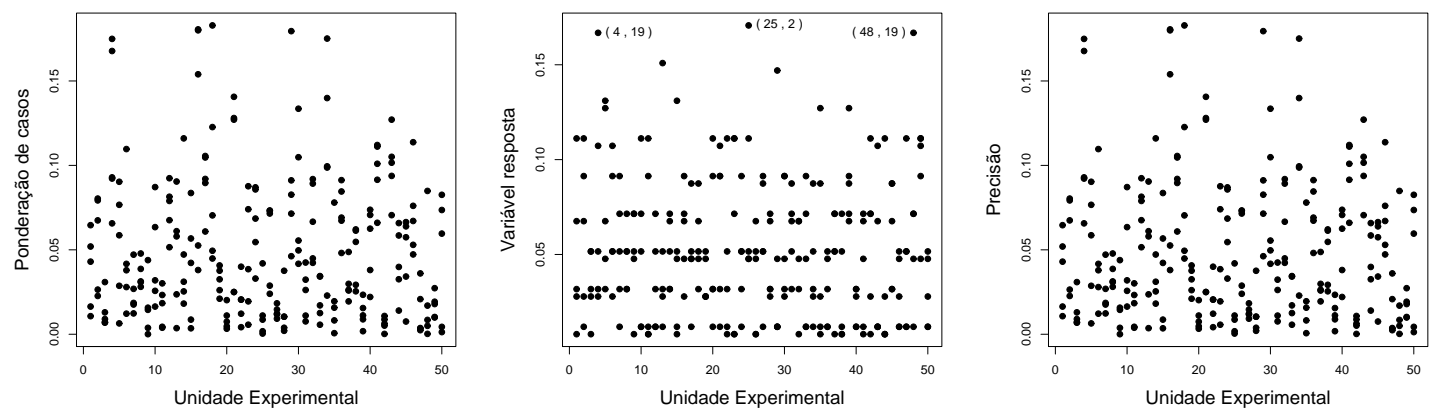

Figura 7.3: Gráficos de influência local considerando a verdadeira matriz de correlação (com estrutura padrão uniforme e $\alpha=0,9$ ) e com matriz de correlação de trabalho padrão uniforme, AR-1 e independente. Simulação Normal sob homogeneidade da dispersão. 

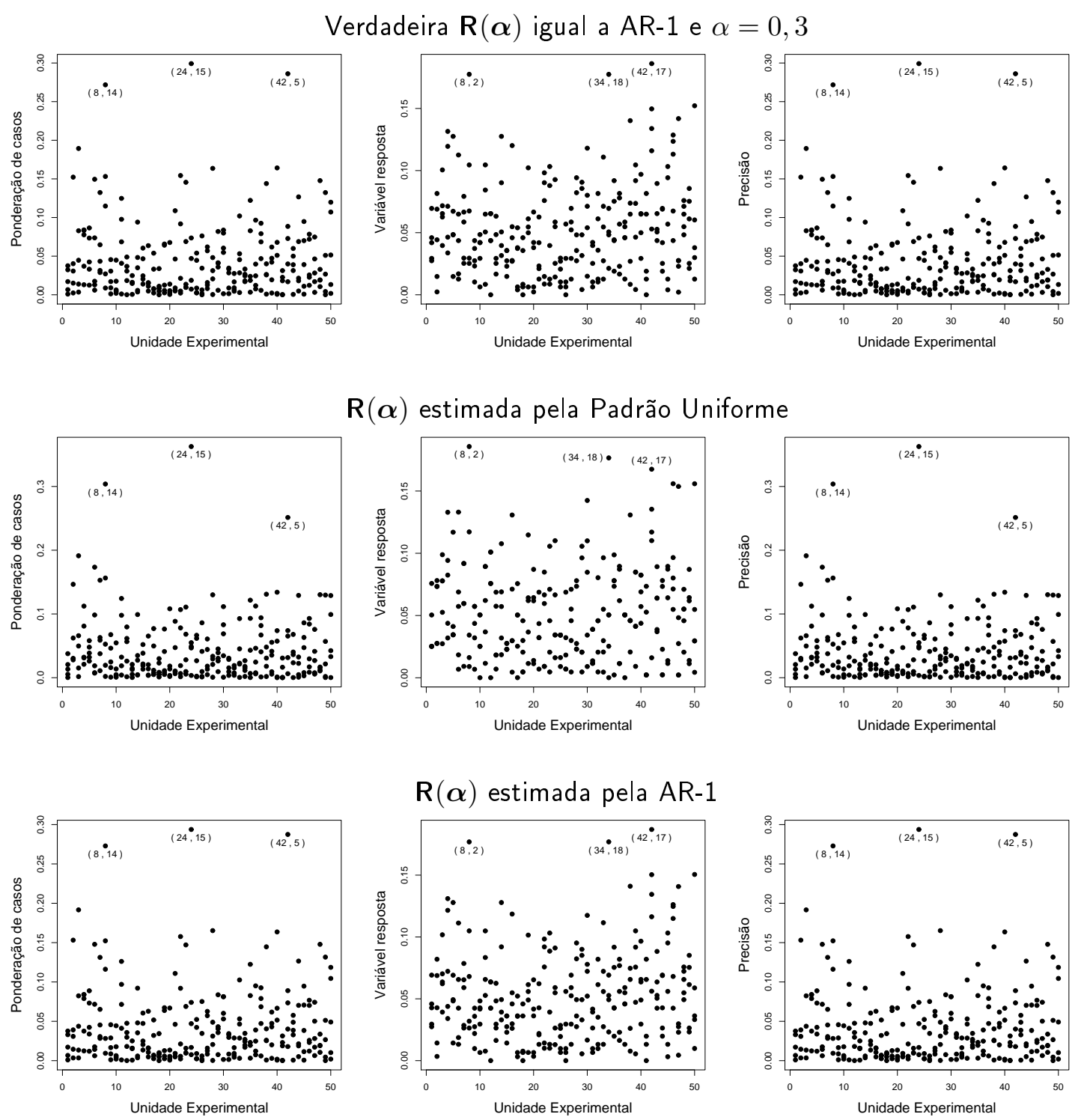

$\mathbf{R}(\boldsymbol{\alpha})$ estimada pela Independente
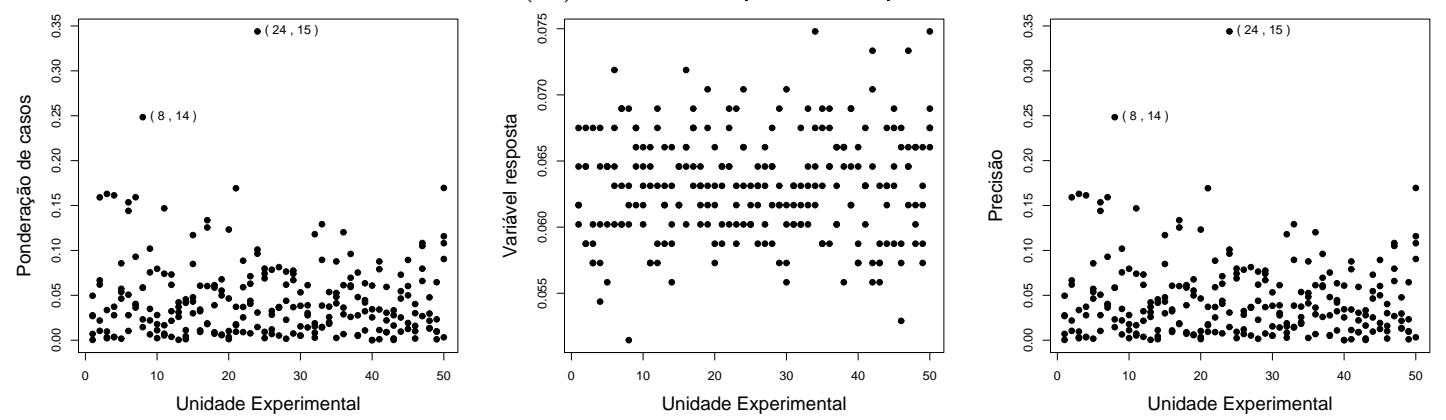

Figura 7.4: Gráficos de influência local considerando a verdadeira matriz de correlação (com estrutura AR-1 e $\alpha=0,3)$ e com matriz de correlação de trabalho padrão uniforme, AR-1 e independente. Simulação Normal sob homogeneidade da dispersão. 

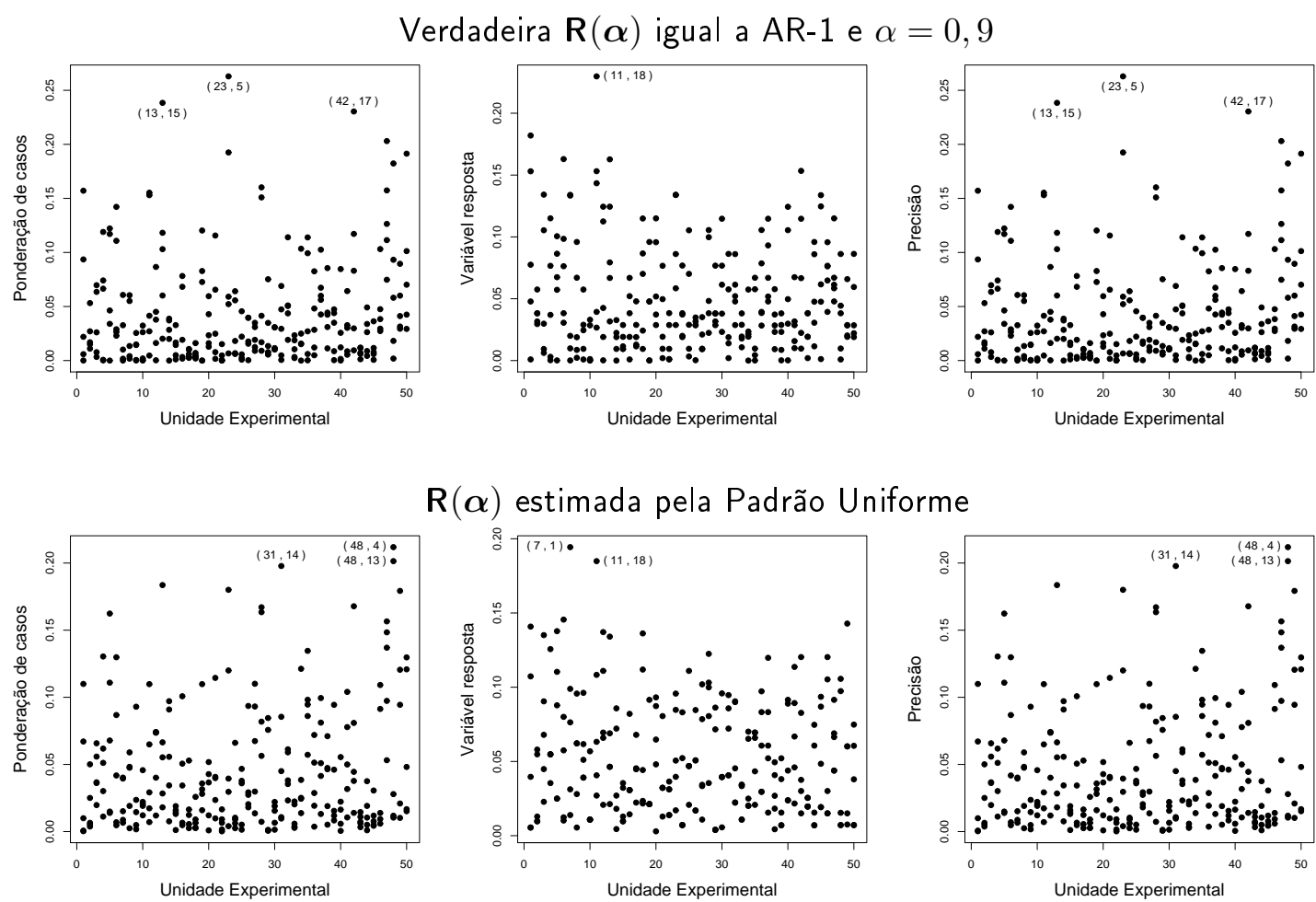

$\mathbf{R}(\boldsymbol{\alpha})$ estimada pela Padrão Uniforme
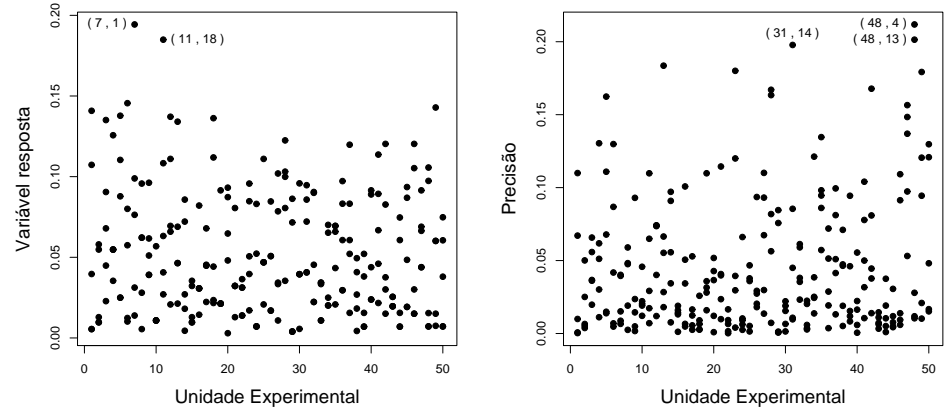

$\mathbf{R}(\boldsymbol{\alpha})$ estimada pela AR-1
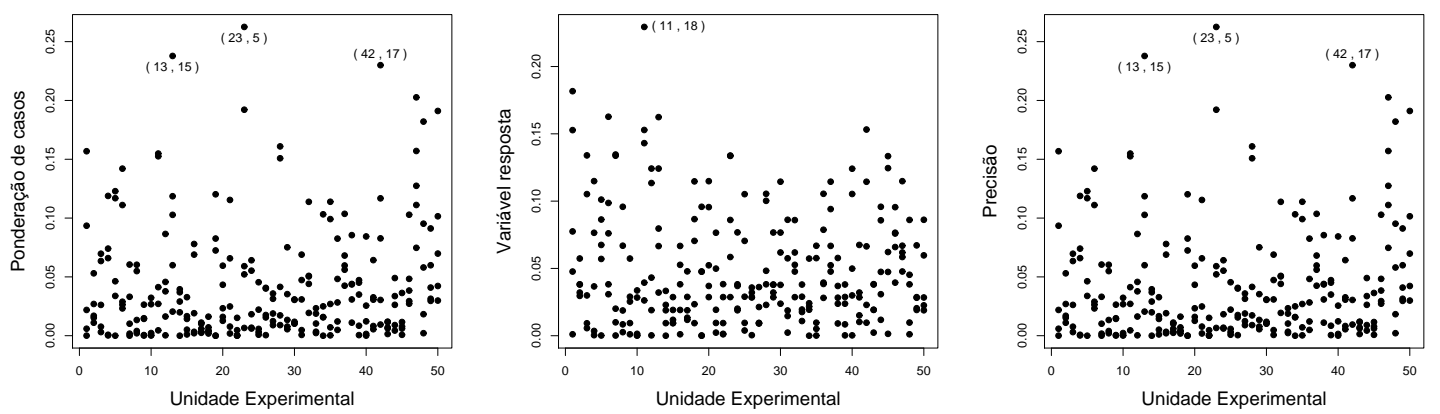

$\mathbf{R}(\boldsymbol{\alpha})$ estimada pela Independente
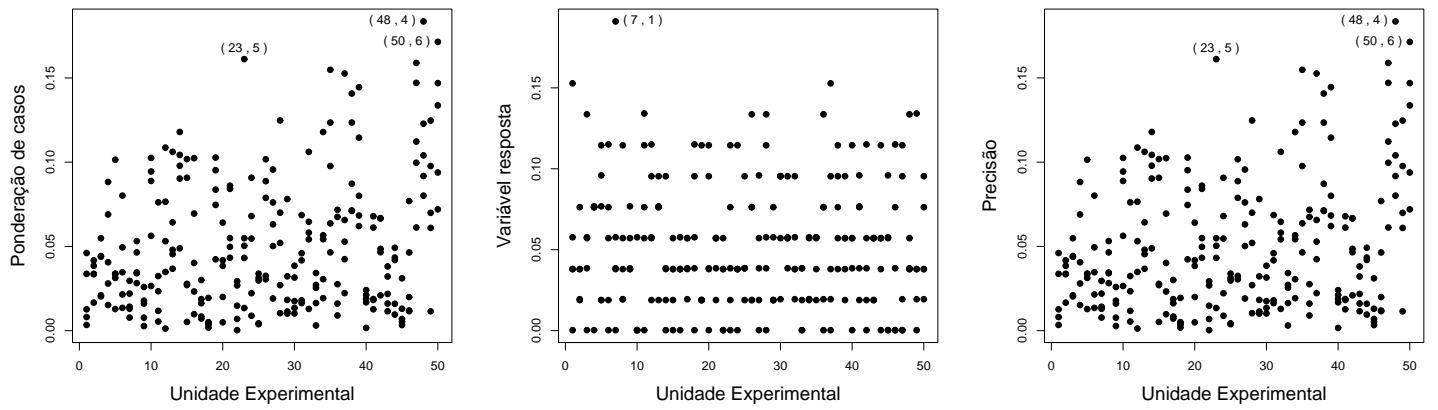

Figura 7.5: Gráficos de influência local considerando a verdadeira matriz de correlação (com estrutura AR-1 e $\alpha=0,9)$ e com matriz de correlação de trabalho padrão uniforme, AR-1 e independente. Simulação Normal sob homogeneidade da dispersão. 
Esses dados foram gerados considerando uma modelagem conjunta da média sob o modelo descrito na equação (7.1) e da dispersão sob o modelo dado por

$$
\log \left(\phi_{i j}^{-1}\right)=-1-0,2 \mathrm{x}_{i j}
$$

sendo $\mathrm{x}_{i j}$ os mesmos valores da covariável gerada no modelo para média, com $i=1, \ldots, 50$ e $j=1, \ldots, 5$.

Nas Figuras 7.6a e 7.7a, temos as variáveis respostas simuladas versus seus respectivos valores da covariável tempo e retas traçadas considerando os verdadeiros valores dos parâmetros de regressão $\left(\beta_{0}=10\right.$ e $\left.\beta_{1}=-1,5\right)$. Nesses gráficos, notamos que a dispersão da variável resposta decresce com o aumento do tempo como conseqüência do modelo de dispersão (7.2) utilizado para gerar os dados.

Cada banco de dados simulado foi ajustado pelo modelo $\mu_{i j}=\beta_{0}+\beta_{1} \mathrm{x}_{i j}$ sob homogeneidade da dispersão, considerando quatro estruturas diferentes para a matriz de correlação de trabalho: a verdadeira matriz de correlação que gerou os dados e com as estruturas padrão uniforme, auto-regressiva de ordem um (AR-1) e independente.

As Figuras 7.6b-e e 7.7b-e apresentam, para esses ajustes, os gráficos de influência local para o esquema de perturbação ponderação de casos. Nesses gráficos, observamos pontos com maior influência nas estimativas dos parâmetros de regressão conforme aumenta o valor predito. Isso provavelmente indica a presença da heterogeneidade do parâmetro de dispersão existente nesses dados simulados.

\subsection{Simulação II - Dados com distribuição de Poisson}

Nesta seção, geramos dados com distribuições marginais de Poisson correlacionadas segundo o algoritmo proposto por Park e Shin (1998) para avaliar o comportamento de algumas medidas de influência local. Pelo mesmo objetivo descrito na seção anterior, simulamos quatro bancos de dados com distribuição de Poisson correlacionadas com as seguintes estruturas:

1. Padrão uniforme com baixa correlação $(\alpha=0,3)$;

2. Padrão uniforme com moderada correlação $(\alpha=0,6)$;

3. AR-1 com baixa correlação $(\alpha=0,3)$; e

4. AR-1 com moderada correlação $(\alpha=0,6)$.

O algoritmo de Park e Shin (1998) apresentou falhas quando consideramos correlações acima de 0,6, impedindo montar um banco de dados com alta correlação. Entretanto, 


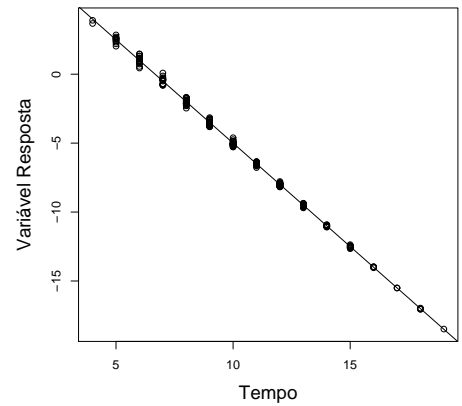

(a)

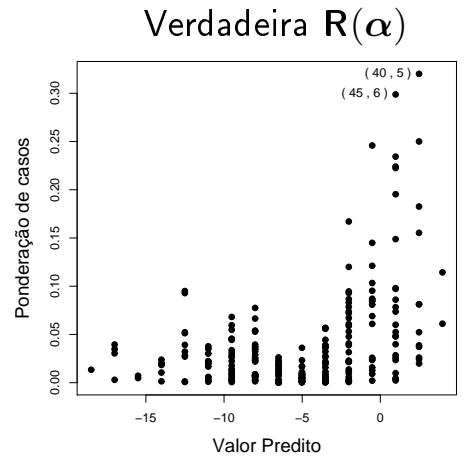

(b)

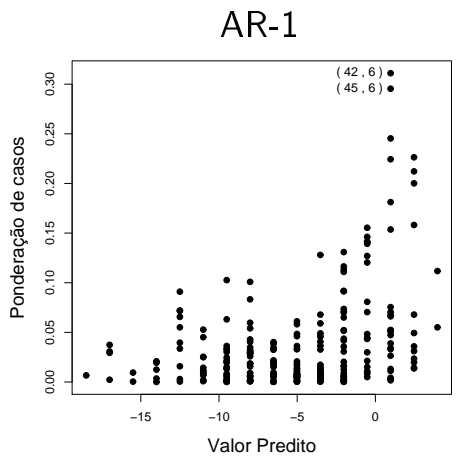

(d)

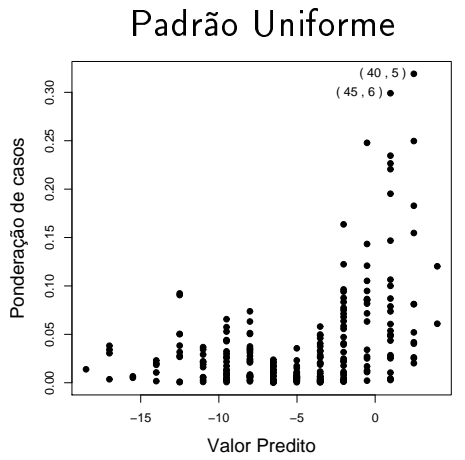

(c)

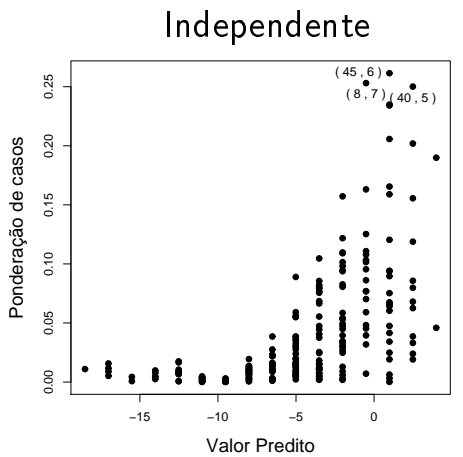

(e)

Figura 7.6: Valores simulados de uma Normal sob heterogeneidade da dispersão e estrutura padrão uniforme com $\alpha=0,9$ (a), medidas de influência local com esquema de perturbação ponderação de casos considerando a verdadeira correlação (b), padrão uniforme (c), AR-1 (d) e independente (e). Simulação Normal sob heterogeneidade da dispersão. 


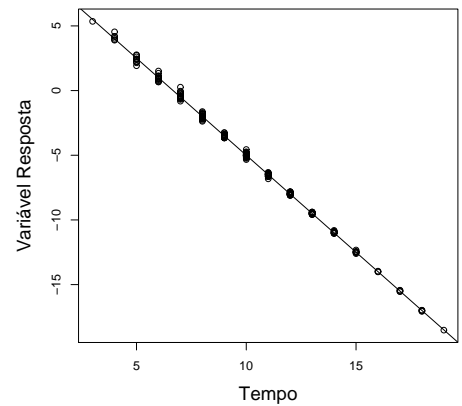

(a)

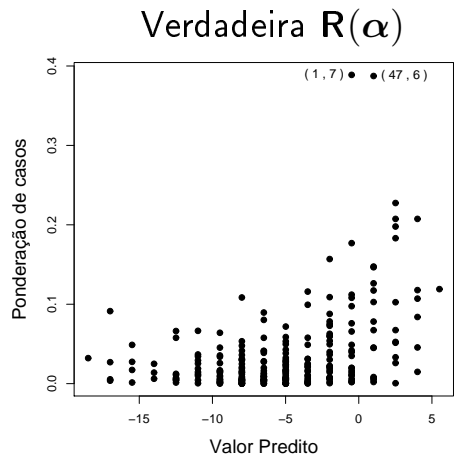

(b)

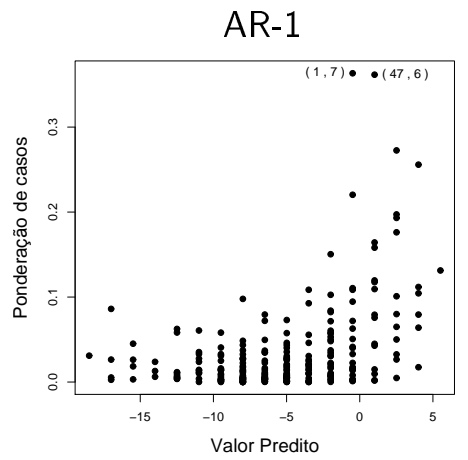

(d)

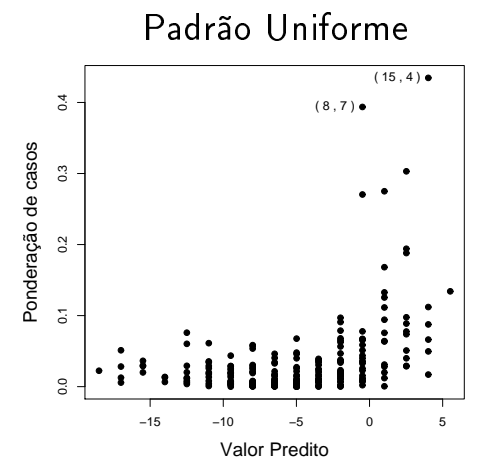

(c)

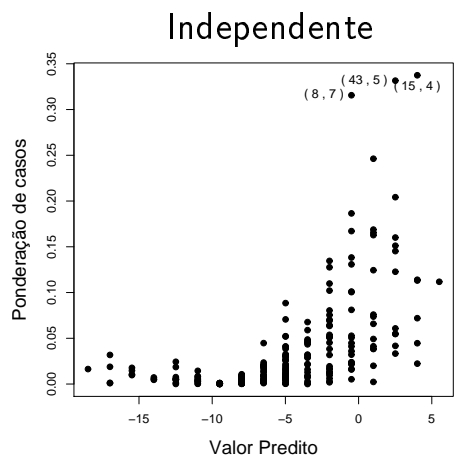

(e)

Figura 7.7: Valores simulados de uma Normal sob heterogeneidade da dispersão e estrutura AR-1 com $\alpha=0,9$ (a), medidas de influência local com esquema de perturbação ponderação de casos considerando a verdadeira correlação (b), padrão uniforme (c), AR-1 (d) e independente (e). Simulação Normal sob heterogeneidade da dispersão. 
veremos a seguir que mesmo trabalhando com uma correlação moderada já temos interpretações semelhantes às descritas para distribuição normal com correlação de 0,9 (ver Seção 7.1).

Para gerar dados com distribuição de Poisson, consideramos o modelo de regressão com ligação canônica dado por

$$
\log \left(\mu_{i j}\right)=3-0,05 \mathrm{x}_{i j}
$$

sendo $\mathrm{x}_{i j}$ valores gerados por uma distribuição uniforme contínua de intervalo $(0 ; 20)$, com $i=1, \ldots, 50$ e $j=1, \ldots, 5$. Lembramos que, na distribuição de Poisson, o parâmetro de dispersão vale um e a função de variância, $\mu_{i j}$.

A Figura 7.8 apresenta gráficos de dispersão das variáveis respostas simuladas versus seus respectivos valores da covariável. As curvas foram traçadas considerando os verdadeiros valores dos parâmetros de regressão $\left(\beta_{0}=3\right.$ e $\left.\beta_{1}=-0,05\right)$. Nesses gráficos, destacamos alguns pontos que serão mencionados na análise das medidas de influência local descrita a seguir.

Cada banco de dados simulado foi ajustado pelo modelo $\log \left(\mu_{i j}\right)=\beta_{0}+\beta_{1} \mathrm{x}_{i j}$ sob homogeneidade da dispersão, considerando quatro estruturas diferentes para a matriz de correlação de trabalho: a verdadeira matriz de correlação que gerou os dados e com as estruturas padrão uniforme, auto-regressiva de ordem um (AR-1) e independente.

As Figuras 7.9 a 7.12 apresentam, para esses ajustes, os gráficos de influência local para os esquemas de perturbação ponderação de casos, da covariável e da precisão. As interpretações obtidas para essas figuras são semelhantes às descritas na seção anterior, na qual os dados foram gerados de normais multivariadas. Os pontos destacados como possíveis influentes são, com certeza, os mesmos apenas quando comparamos o ajuste que utiliza a verdadeira matriz de correlação que gerou os dados com o que utiliza uma estrutura de correlação igual à essa verdadeira matriz de correlação. Quando se considera no ajuste uma estrutura de correlação de trabalho diferente da estrutura da verdadeira matriz de correlação, isso tende a ocorrer apenas quando a correlação entre as observações for baixa.

Analisando as medidas de influência local obtidas dos bancos simulados com baixa correlação, observamos os seguintes conjuntos de observações se destacando como mais influentes no ajuste que considera a verdadeira matriz de correlação: $(1,4),(7,1)$ e $(11,3)$ na Figura 7.9 e, na Figura 7.11, os pontos $(5,4),(14,5),(17,5),(28,2)$ e $(37,5)$. Esses pontos representam os índices da unidade experimental $i$ e da medida repetida $j$, ou seja, $(i, j)$. Apesar de poder alterar a grandeza da medida de influência local, esses conjuntos de observações também se destacam nos gráficos de influência obtidos dos ajustes que 
Verdadeira $\mathbf{R}(\boldsymbol{\alpha})$ igual a Padrão Uniforme

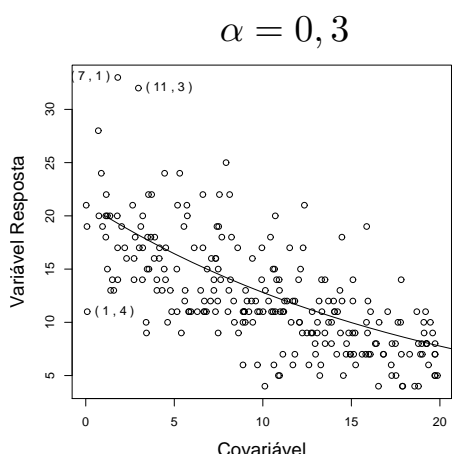

(a)

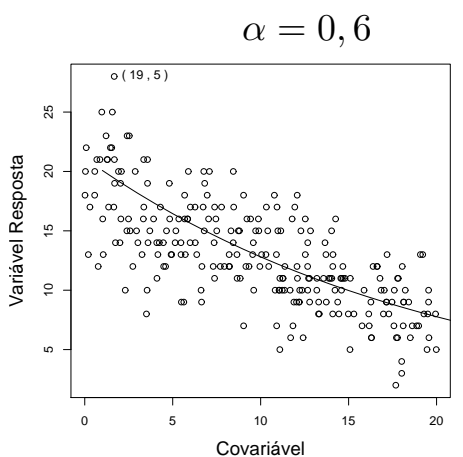

(b)

Verdadeira $\mathbf{R}(\boldsymbol{\alpha})$ igual a AR-1

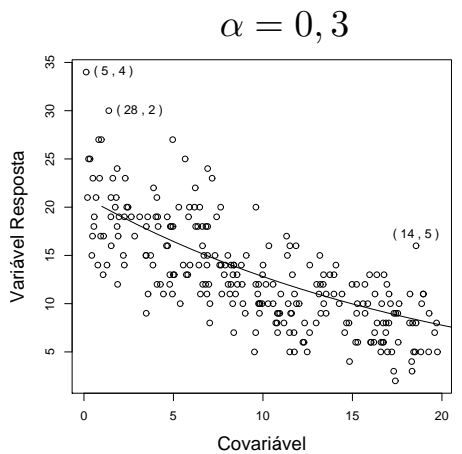

(c)

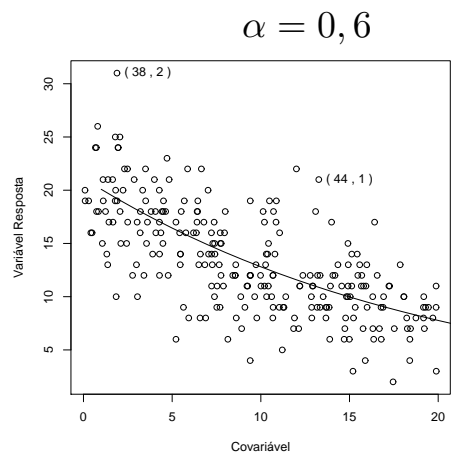

(d)

Figura 7.8: Valores simulados de uma Poisson considerando estrutura padrão uniforme com $\alpha=0,3$ (a) e $\operatorname{com} \alpha=0,6$ (b) e estrutura AR-1 $\operatorname{com} \alpha=0,3$ (c) e com $\alpha=0,6$ (d). Simulação Poisson.

consideram as estruturas padrão uniforme, AR-1 e independente.

Nas Figuras 7.10 e 7.12, cujos dados foram gerados com alta correlação entre as observações da mesma unidade experimental, temos exatamente as mesmas conclusões com relação às observações que se destacam como possíveis pontos influentes apenas quando o ajuste tem estrutura de correlação igual à da verdadeira matriz de correlação que gerou os dados. 
Verdadeira $\mathbf{R}(\boldsymbol{\alpha})$ igual a Padrão Uniforme e $\alpha=0,3$
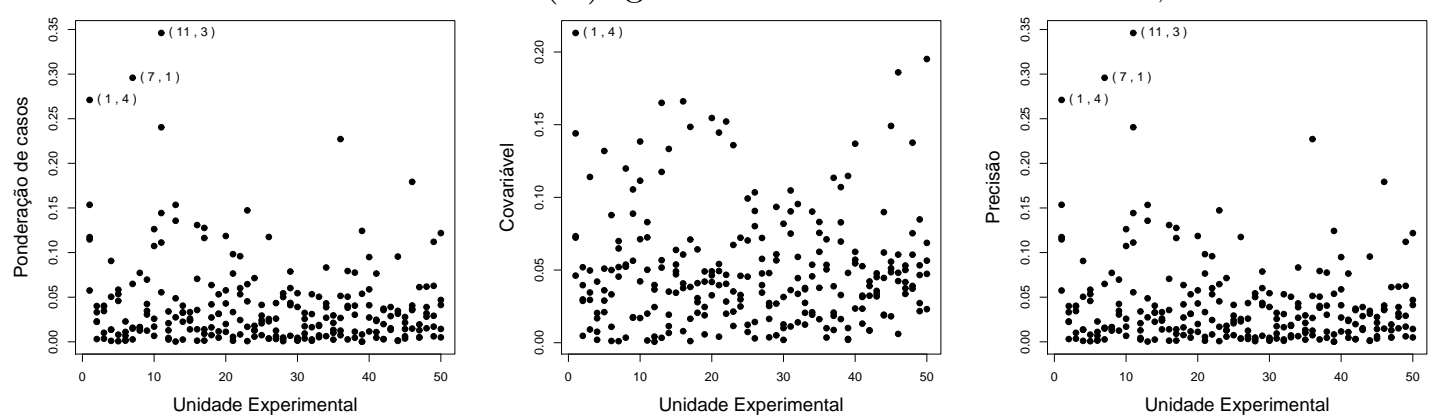

$\mathbf{R}(\boldsymbol{\alpha})$ estimada pela Padrão Uniforme
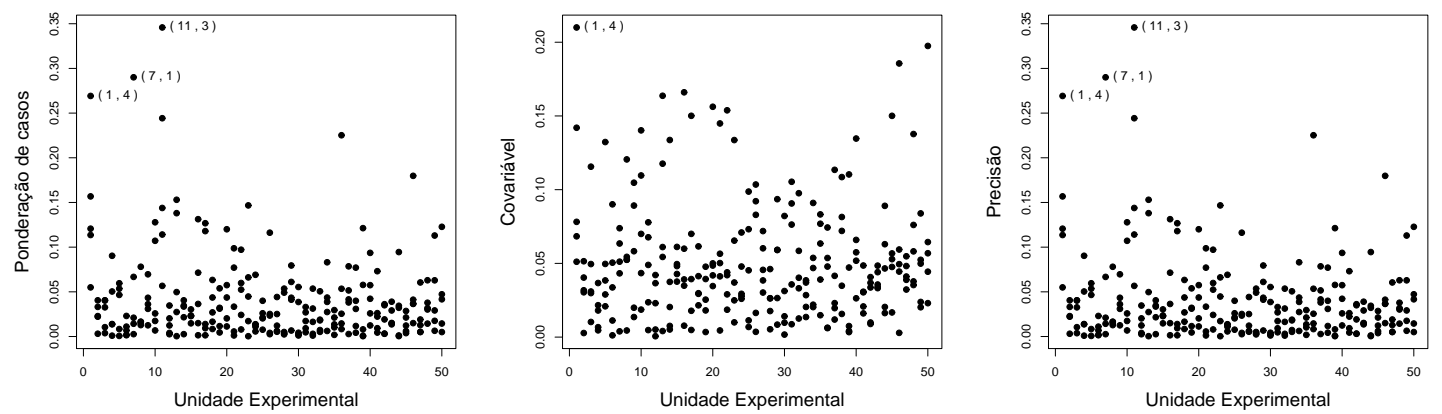

$\mathbf{R}(\boldsymbol{\alpha})$ estimada pela AR-1
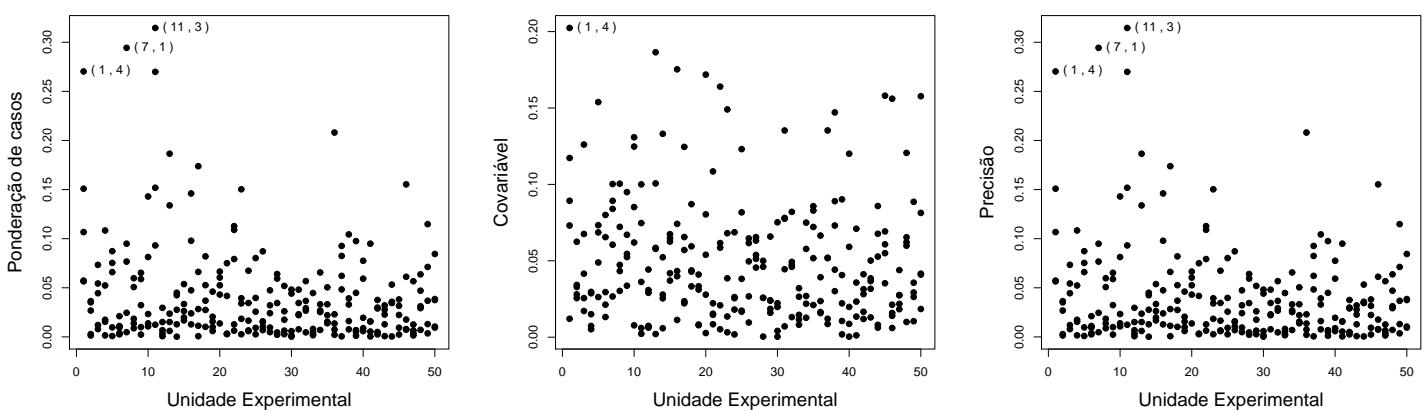

$\mathbf{R}(\boldsymbol{\alpha})$ estimada pela Independente
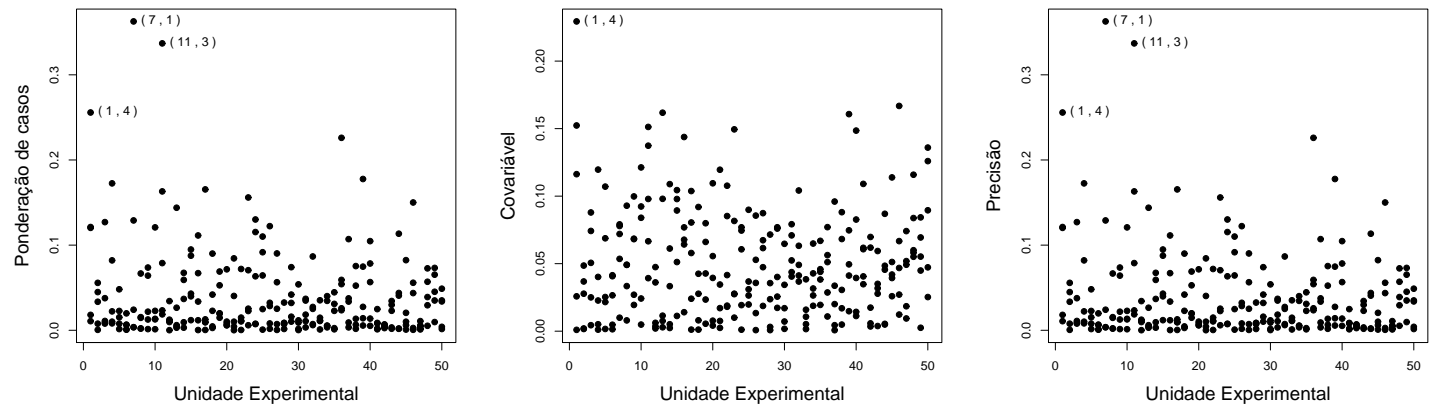

Figura 7.9: Gráficos de influência local considerando a verdadeira matriz de correlação (com estrutura padrão uniforme e $\alpha=0,3$ ) e com matriz de correlação de trabalho padrão uniforme, AR-1 e independente. Simulação Poisson. 
Verdadeira $\mathbf{R}(\boldsymbol{\alpha})$ igual a Padrão Uniforme e $\alpha=0,6$
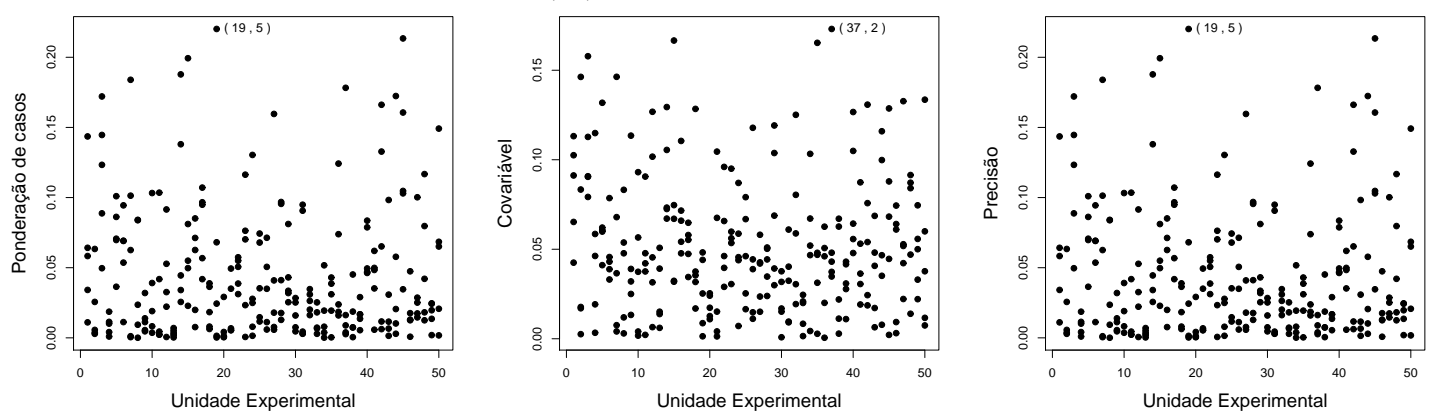

$\mathbf{R}(\boldsymbol{\alpha})$ estimada pela Padrão Uniforme
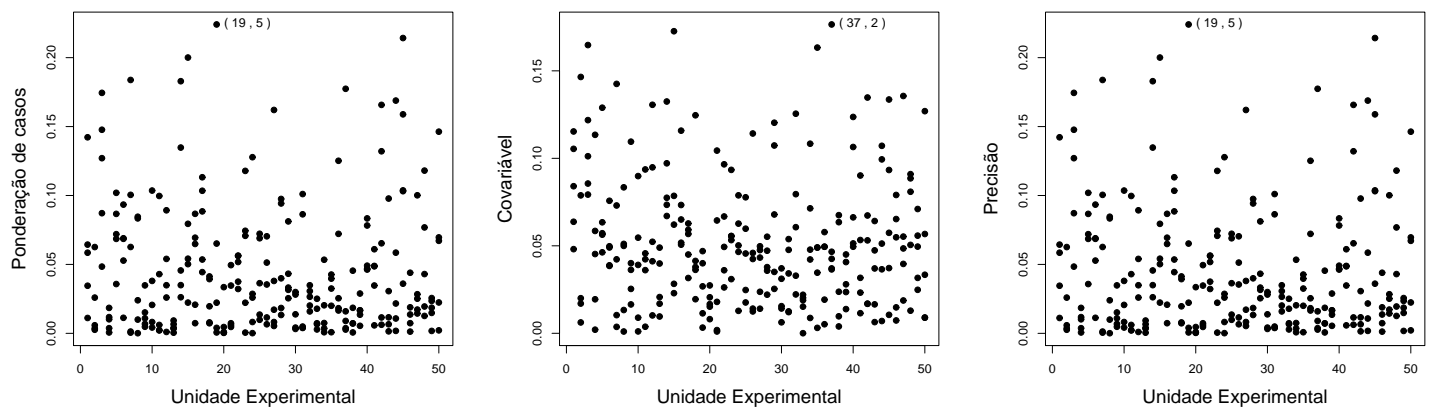

$\mathbf{R}(\boldsymbol{\alpha})$ estimada pela AR-1
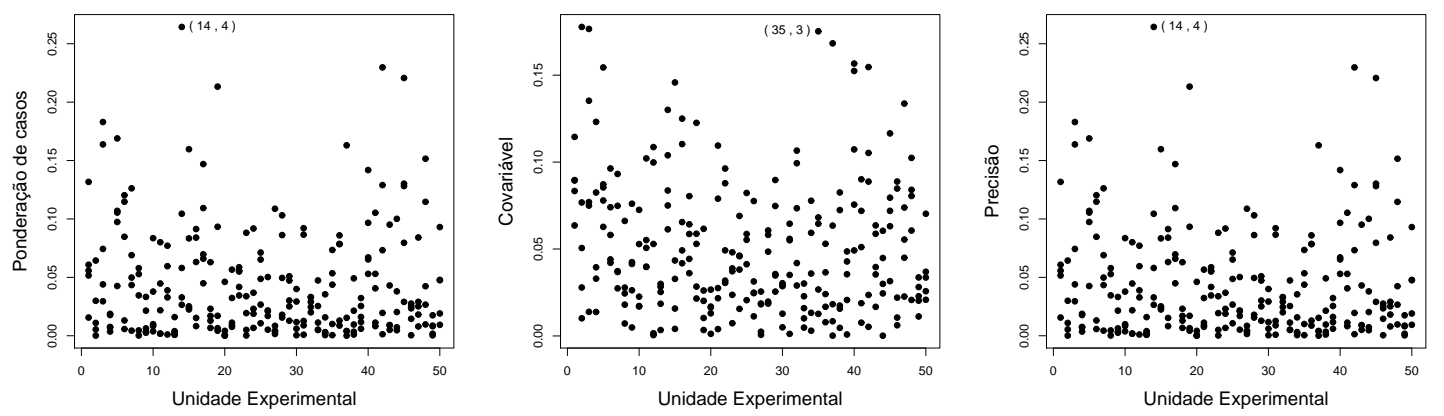

$\mathbf{R}(\boldsymbol{\alpha})$ estimada pela Independente
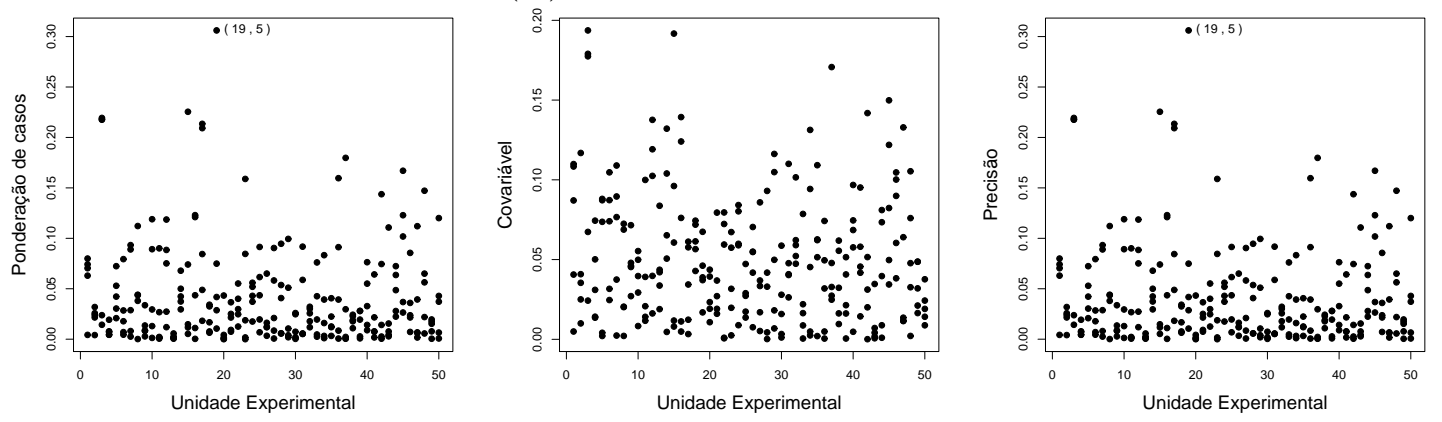

Figura 7.10: Gráficos de influência local considerando a verdadeira matriz de correlação (com estrutura padrão uniforme e $\alpha=0,6$ ) e com matriz de correlação de trabalho padrão uniforme, AR-1 e independente. Simulação Poisson. 
Verdadeira $\mathbf{R}(\boldsymbol{\alpha})$ igual a AR-1 e $\alpha=0,3$
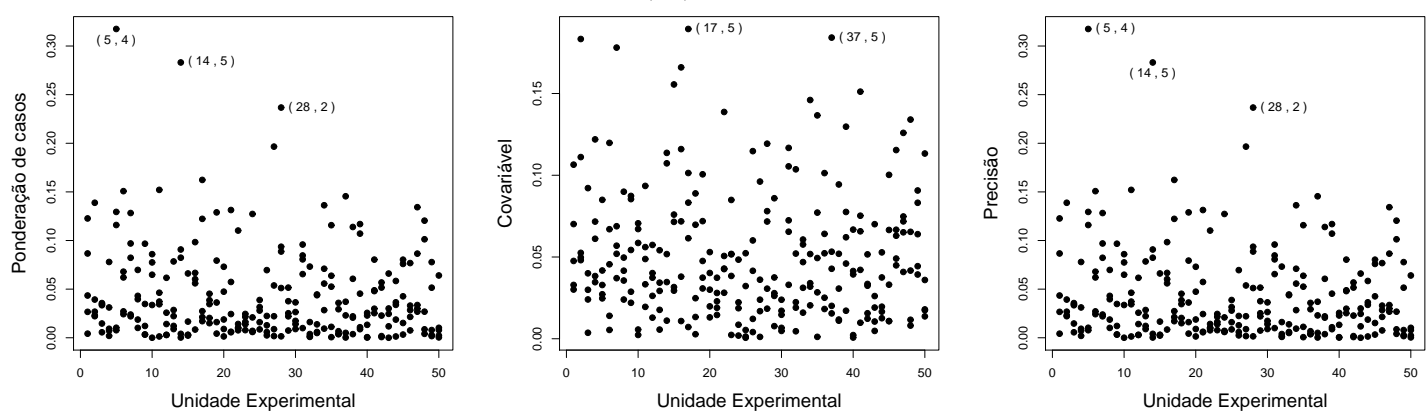

$\mathbf{R}(\boldsymbol{\alpha})$ estimada pela Padrão Uniforme
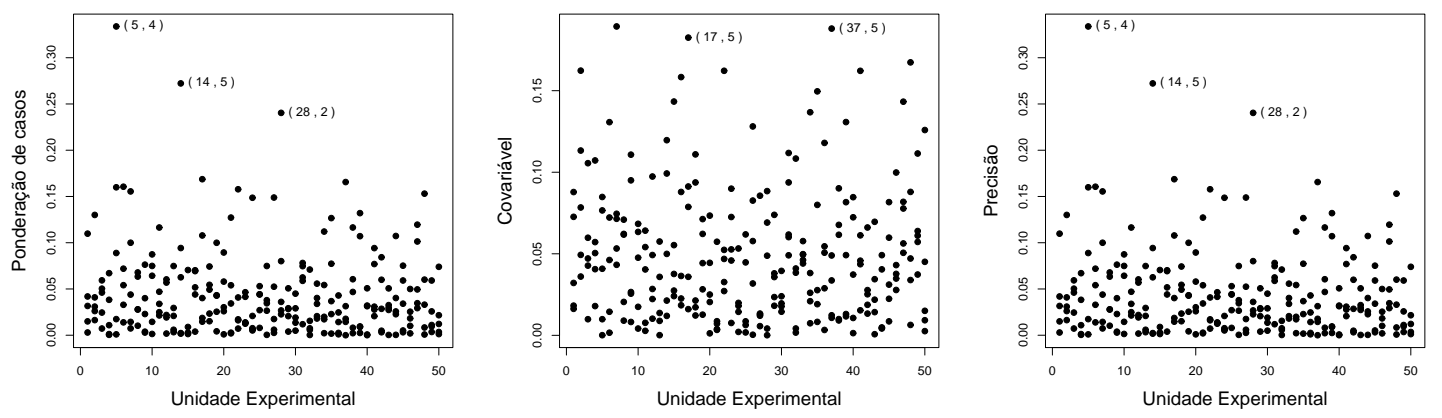

$\mathbf{R}(\boldsymbol{\alpha})$ estimada pela AR-1
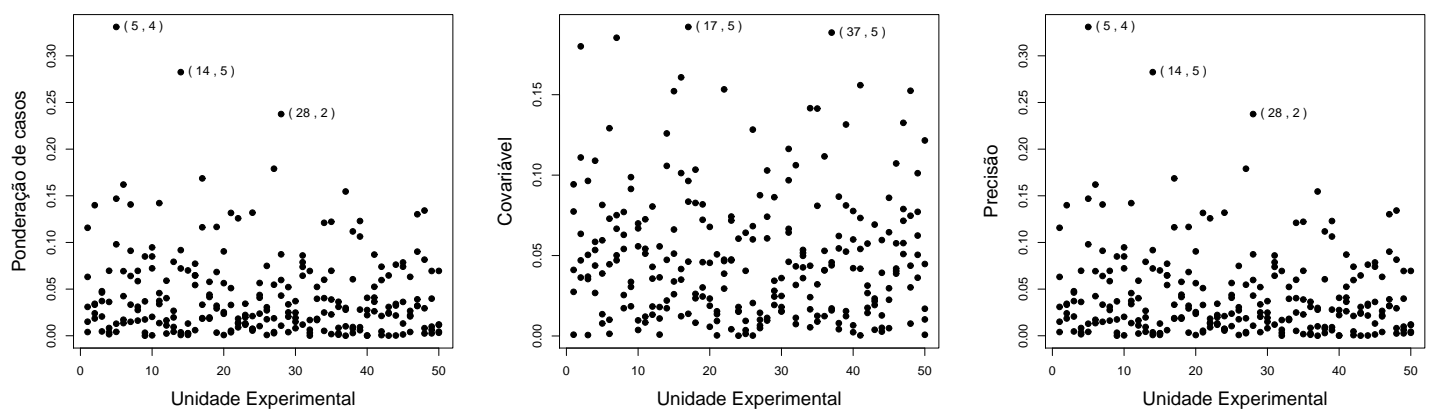

$\mathbf{R}(\boldsymbol{\alpha})$ estimada pela Independente
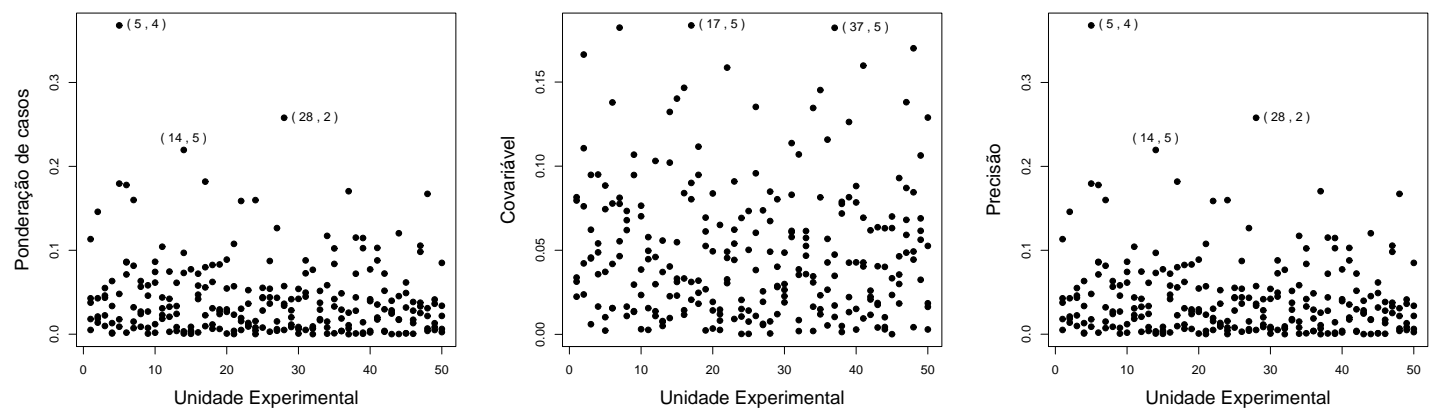

Figura 7.11: Gráficos de influência local considerando a verdadeira matriz de correlação (com estrutura AR-1 e $\alpha=0,3)$ e com matriz de correlação de trabalho padrão uniforme, AR-1 e independente. Simulação Poisson. 


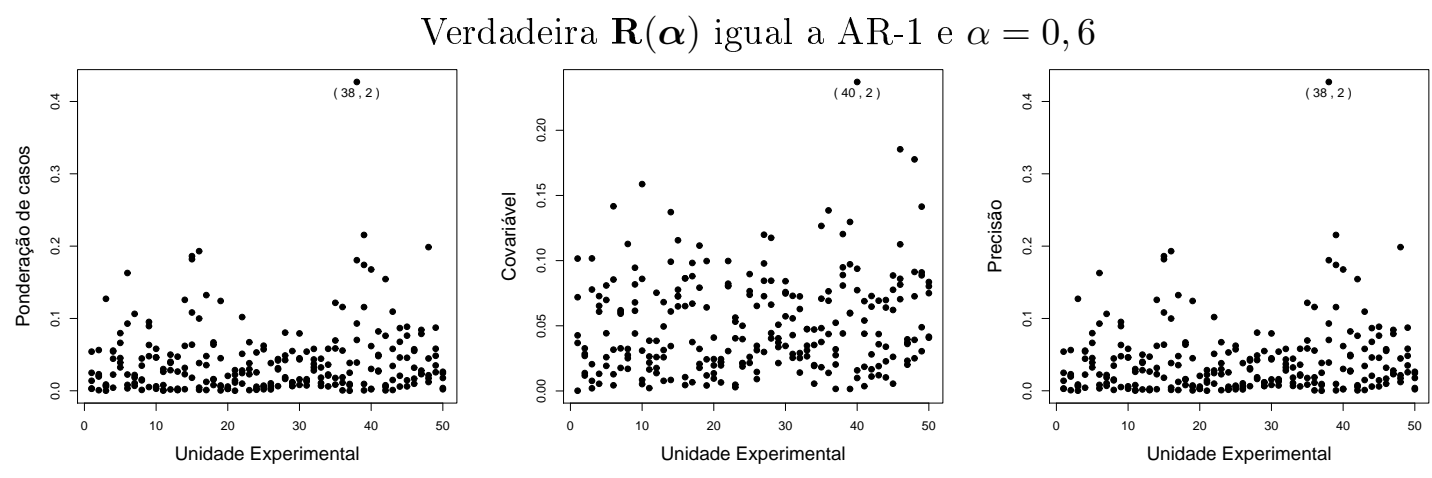

$\mathbf{R}(\boldsymbol{\alpha})$ estimada pela Padrão Uniforme
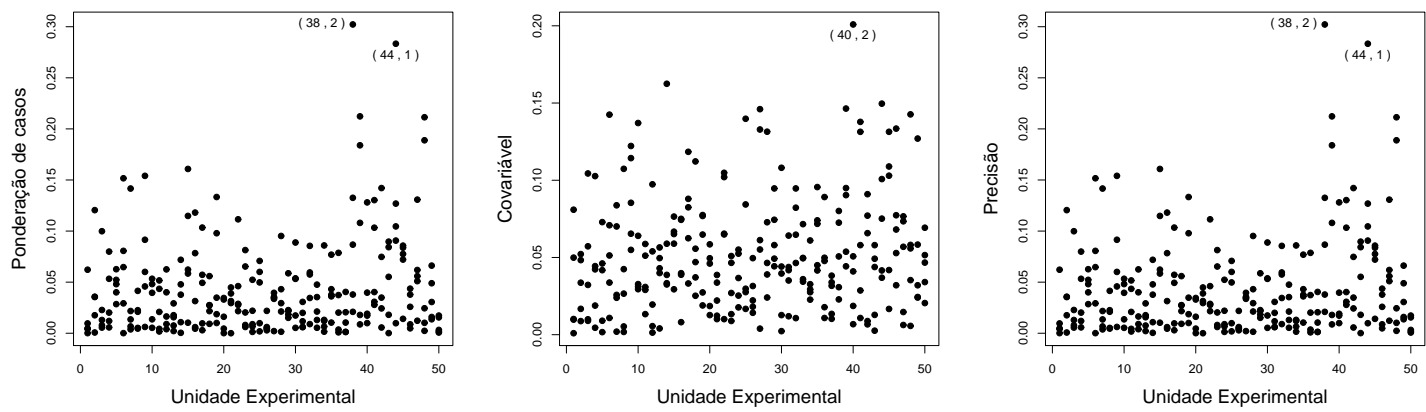

$\mathbf{R}(\boldsymbol{\alpha})$ estimada pela AR-1
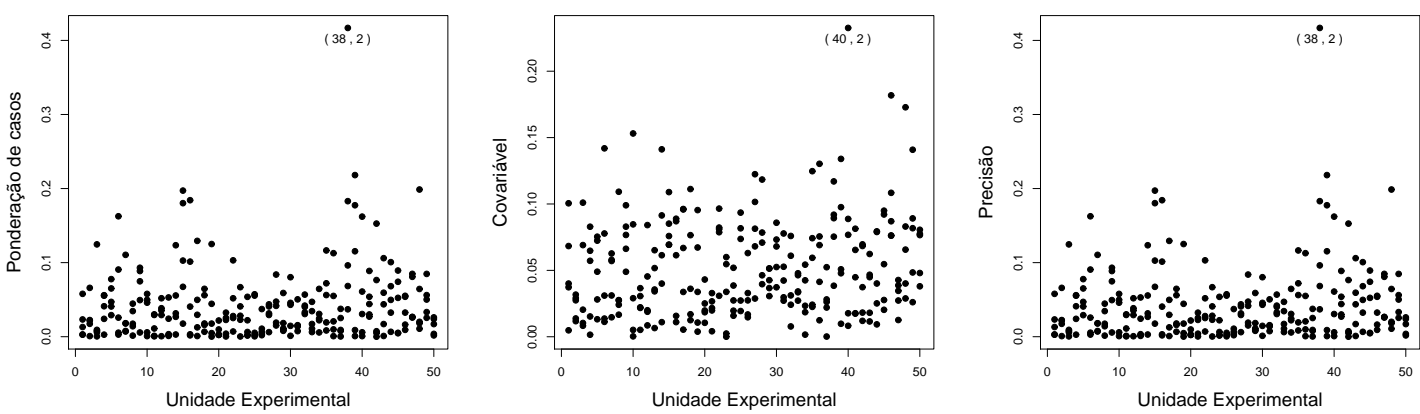

$\mathbf{R}(\boldsymbol{\alpha})$ estimada pela Independente
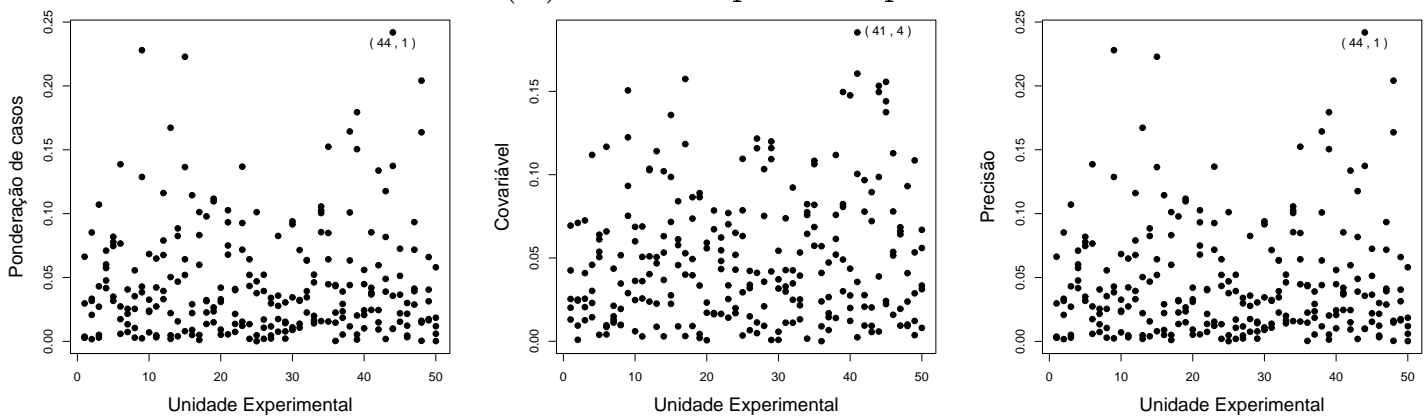

Figura 7.12: Gráficos de influência local considerando a verdadeira matriz de correlação (com estrutura AR-1 e $\alpha=0,6$ ) e com matriz de correlação de trabalho padrão uniforme, AR-1 e independente. Simulação Poisson. 


\section{Capítulo 8}

\section{Aplicações a dados reais}

Neste capítulo, analisamos dois conjuntos de dados utilizando o método de equações de estimação generalizadas detalhado na Parte I e as técnicas de diagnóstico apresentadas na Parte II.

\subsection{Aplicação I - Estudo de oftalmologia}

Esta aplicação se refere a um estudo de oftalmologia apresentado por Meyers et al. (1992) que avalia o uso de gás intraocular em reparação cirúrgica da retina. No momento da cirurgia, um determinado volume de gás intraocular, em três diferentes concentrações, foi injetado nos olhos de 31 pacientes, tendo: 7 pacientes com concentração de gás igual a 15; 14 pacientes com concentração de gás igual a 20; e 10 pacientes com concentração de gás igual a 25 .

Esses pacientes foram observados de 3 a 13 vezes num período de três meses e, em cada visita, foi registrada a porcentagem de volume de gás ainda presente nos olhos com relação ao volume de gás injetado no momento da cirurgia ${ }^{1}$. A Figura 8.1 apresenta o comportamento dessa variável ao longo do tempo após a cirurgia (em dias), para as três concentrações de gás.

\section{BETA}

Inicialmente, ajustamos um modelo com medidas repetidas para variável resposta considerando distribuição beta, função de ligação complemento log-log e homogeneidade da dispersão, conforme teoria apresentada na Seção 2.1. A média desse modelo é dada

\footnotetext{
${ }^{1}$ Para valores da variável resposta iguais a 0 e 1 consideramos, respectivamente, iguais a 0, 001 e 0,999.
} 


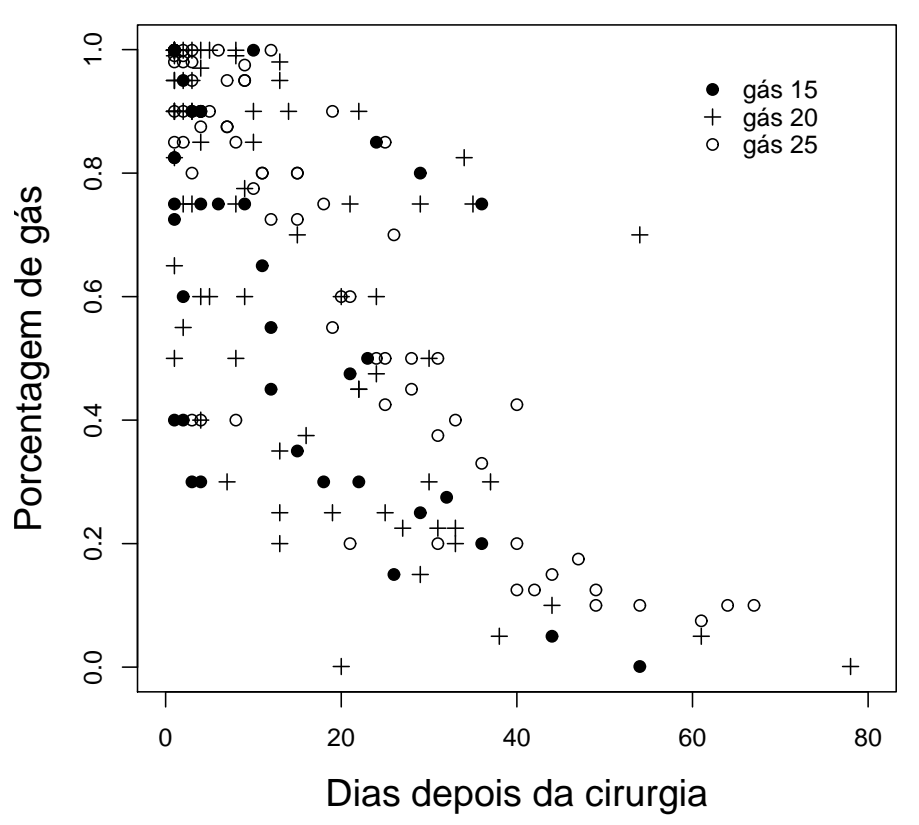

Figura 8.1: Gráfico de dispersão da porcentagem de gás versus dias após a cirurgia, para as concentrações de gás iguais a 15, 20 e 25. Estudo de oftalmogia.

por

$$
\log \left[-\log \left(1-\mu_{i j}\right)\right]=\beta_{0}+\beta_{1} \log \left(\mathrm{x}_{1 i j}\right)+\beta_{2} \log ^{2}\left(\mathrm{x}_{1 i j}\right)+\beta_{3}\left(\mathrm{x}_{2 i j}\right),
$$

sendo $\mathrm{x}_{1 i j}$ o tempo após a cirurgia (em dias) e

$$
\mathrm{x}_{2 i j}=\frac{\operatorname{gas}_{i j}-20}{5}=\left\{\begin{array}{rl}
-1 & \text { se a concentração de gás é } 15 \\
0 & \text { se a concentração de gás é } 20 \\
1 & \text { se a concentração de gás é } 25
\end{array} .\right.
$$

De acordo com a medida $Q I C$ apresentada na equação (5.8), o ajuste com a estrutura AR-1 foi escolhido como o mais adequado para explicar a correlação entre as respostas da mesma unidade experimental. A função de ligação complemento log-log foi escolhida por permitir uma curva com comportamentos distintos para $F(\mathrm{y}) \leq 0,5$ e $F(\mathrm{y})>0,5$, diferente da função de ligação logito que é simétrica em torno de $F(\mathrm{y})=0,5$ (Paula, 2004, p.7). As estimativas dos parâmetros desse modelo estão apresentadas na Tabela 8.1 e as curvas ajustadas, na Figura 8.2a. Nessa tabela, observamos que apenas o coeficiente do quadrado do logaritmo neperiano do tempo, $\log ^{2}\left(x_{1}\right)$, é significante para descrever, em média, a queda do volume de gás ainda presente nos olhos dos pacientes (nível descritivo, $p<0,001)$. 
Tabela 8.1: Estimativas e erros padrões dos parâmetros do modelo de regressão beta sob homogeneidade da dispersão e com estrutura AR-1. Estudo de oftalmologia.

\begin{tabular}{cccccr}
\hline & & \multicolumn{3}{c}{ Erro Padrão } & \multicolumn{1}{c}{ Nível } \\
\multicolumn{2}{c}{ Parâmetro } & Estimativa & Robusto & Naive & Descritivo \\
\hline$\beta_{0}$ & Intercepto & 0,7386 & 0,0782 & 0,0796 & $<0,001$ \\
$\beta_{1}$ & $\log \left(\mathrm{x}_{1}\right)$ & 0,1435 & 0,0834 & 0,0834 & 0,085 \\
$\beta_{2}$ & $\log ^{2}\left(\mathrm{x}_{1}\right)$ & $-0,1687$ & 0,0228 & 0,0279 & $<0,001$ \\
$\beta_{3}$ & $\mathrm{x}_{2}$ & 0,1556 & 0,1150 & 0,0702 & 0,176 \\
$\phi$ & Precisão & 2,9195 & & & \\
$\alpha$ & Correlação & 0,7096 & & & \\
\hline
\end{tabular}

Aplicando aos dados as técnicas de diagnóstico apresentadas no Capítulo 5, calculamos a medida de alavanca baseada na matriz de projeção, a distância de Cook e o resíduo padronizado cujos valores estão apresentados nas Figuras 8.2b-d, respectivamente. Na primeira figura citada, não temos observações se destacando como alavanca. Na Figura 8.2c, temos que a distância de Cook do paciente 16 com dez dias após a cirurgia, $(16,10)$, é bem maior do que a das demais observações e um grupo de observações $((2,1),(9,12)$, $(16,1)$ e $(25,20))$ tem distâncias de Cook menores do que a da observação $(16,10)$, mas também se destancando das demais observações, indicando serem possíveis pontos influentes. Na Figura 8.2d, temos que os resíduos padronizados do paciente 16 com dez dias após a cirurgia, $(16,10)$ e do paciente 25 com vinte dias após a cirurgia,$(25,20)$, são maiores do que os das demais observações, indicando serem também possíveis pontos aberrantes.

Nas Figuras 8.3a-d, encontramos os gráficos de influência local, respectivamente, para os seguintes esquemas de perturbação: ponderação de casos, da variável resposta, da covariável logaritmo neperiano do tempo $\left(\log \left(\mathrm{x}_{1}\right)\right)$ e da precisão. Ressaltamos que o esquema de perturbação da covariável concentração de gás $\left(\mathrm{x}_{2}\right)$ é inviável por se tratar de uma covariável categorizada. Analisando de forma geral esses gráficos de influência local, temos a presença dos pontos $(2,1),(16,10)$ e $(25,20)$ se destacando como influentes em pelo menos um dos quatro esquemas de perturbação. Além disso, também podemos notar que observações com valores preditos superiores a 0,8 exercem maior influência no esquema de perturbação de ponderação de casos, da variável resposta e da precisão, sendo um indicativo de presença de heterogeneidade do parâmetro de precisão.

No banco de dados, vemos que as observações $(2,1)$ e $(16,10)$ pertencem ao grupo com 
concentração de gás 15 e que a porcentagem de volume presente no primeiro e no décimo dias após a cirurgia no caso, respectivamente, dos pacientes 2 e 16 ainda é a máxima. Na Figura 8.1, observamos que a maioria das observações pertencentes à concentração de gás 15 já sofreu uma redução de pelo menos $10 \%$ no volume de gás no primeiro dia após a cirurgia. A observação $(25,20)$ pertence à concentração de gás 20 e sua porcentagem de volume de gás é nula, evento atípico se a compararmos com as demais observações pertencentes a essa concentração de gás.

Ajustando novamente o modelo (8.1), mas sem as observações $(2,1),(16,10)$ e $(25,20)$, notamos que as curvas ainda não se ajustam bem aos dados (ver Figura 8.2e). Os gráficos de influência local, Figuras 8.3e-h, destacam novos pontos como influentes e continua o indício de possível heterogeneidade do parâmetro de precisão. As variações percentuais nas estimativas dos parâmetros devido à exclusão dessas três observações influentes afetam mais as estimativas de $\beta_{1}$ e de $\beta_{3}$ aumentando $10,2 \%$ e $29,4 \%$, respectivamente, causando uma redução em seus níveis descritivos para $p=0,054$ e $p=0,033$. A exclusão dessas observações altera a conclusão da covariável concentração de gás que passa a ser significativa.

Para avaliarmos a possível presença de heterogeneidade da precisão, conforme descrito anteriormente, fizemos uma modelagem conjunta da média sob o modelo (8.1) e da precisão sob o modelo:

$$
\log \left(\phi_{i j}\right)=\gamma_{0}+\gamma_{1}\left(\mathrm{x}_{2 i j}\right)
$$

Nesse ajuste, também consideramos a estrutura AR-1 para explicar a correlação entre as respostas da mesma unidade experimental. As estimativas dos parâmetros desse modelo estão na Tabela 8.2 e apenas as referentes aos parâmetros de precisão são significativas (nível descritivo, $p<0,001$ ). As curvas ajustadas estão na Figura 8.4a e se mostram melhor ajustadas do que as obtidas pelos modelos sob homogeneidade da precisão (ver Figuras $8.2 \mathrm{a}$ e $8.2 \mathrm{e})$.

As Figuras $8.4 \mathrm{~b}-\mathrm{g}$ apresentam a medida de alavanca baseada na matriz de projeção, a distância de Cook e o resíduo padronizado para detectar pontos alavancas, influentes e/ou aberrantes nos ajustes da média $(\boldsymbol{\beta})$ e da precisão $(\boldsymbol{\gamma})$. Analisando a Figura 8.4c, temos o ponto $(9,12)$ se destacando como possível ponto influente no ajuste de $\boldsymbol{\beta}$. Quanto ao ajuste de $\gamma$, temos quatro observações do paciente 16 como possíveis pontos influentes (ver Figura 8.4f) e os pontos $(16,10)$ e $(25,20)$ como pontos aberrantes (ver Figura 8.4g).

Na Figura 8.5, apresentamos as medidas de influência local para os seguintes esquemas de perturbação: ponderação de casos, da variável resposta e da covariável $\log \left(\mathrm{x}_{1}\right)$. Neste último caso, utilizamos o cenário descrito na Seção 6.4.3.1 que considera a matriz que 


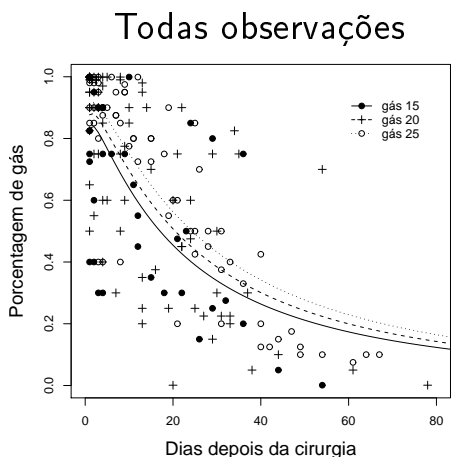

(a)

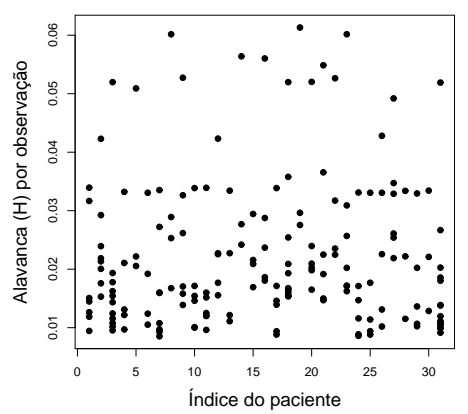

(b)

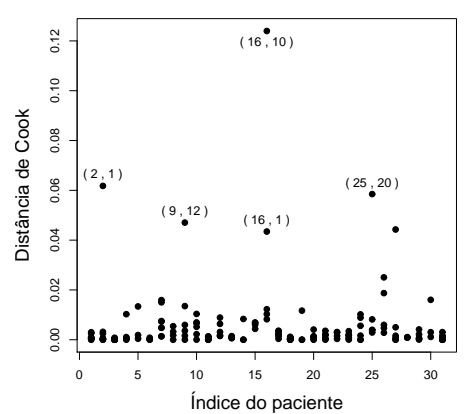

(c)

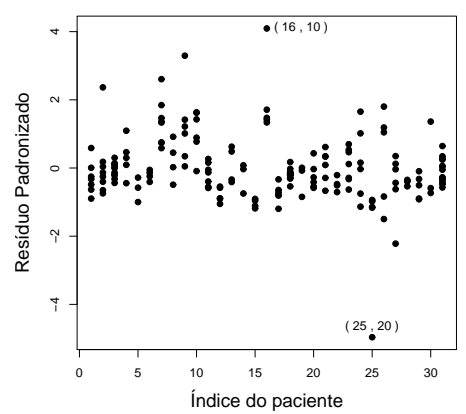

(d)

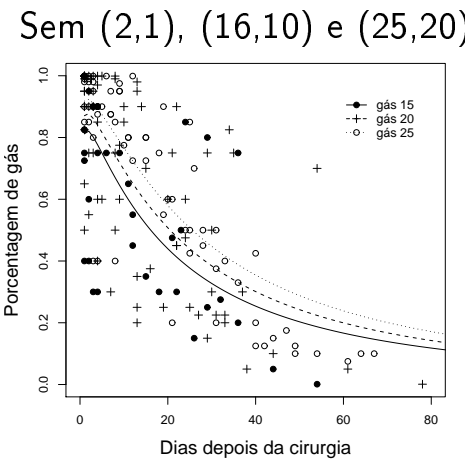

(e)

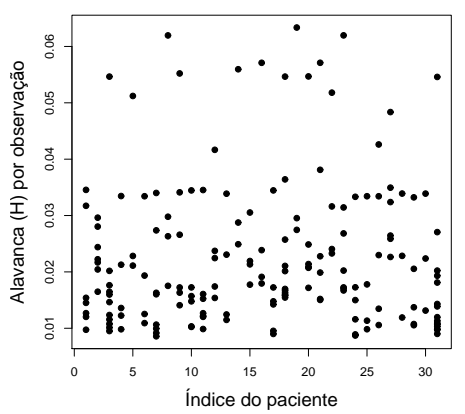

(f)

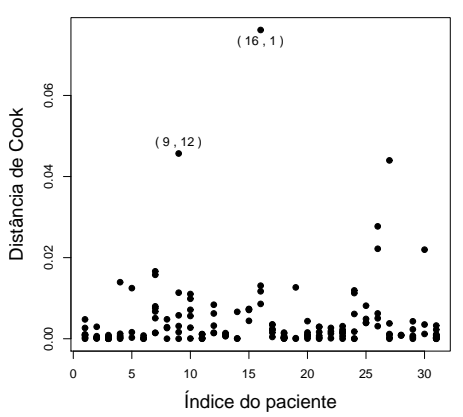

(g)

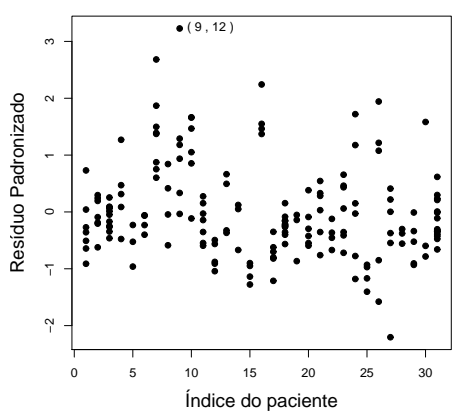

(h)

Figura 8.2: Curvas ajustadas e gráficos de diagnóstico com todas as observações (a)-(d) e sem as observações $(2,1),(16,10)$ e $(25,20)$ (e)-(h), para modelo de regressão beta sob homogeneidade da dispersão. Estudo de oftalmologia. 


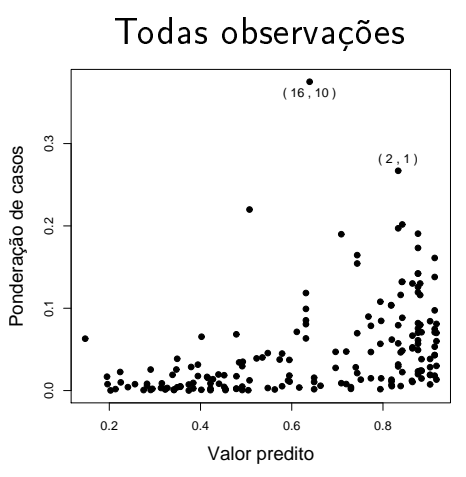

(a)

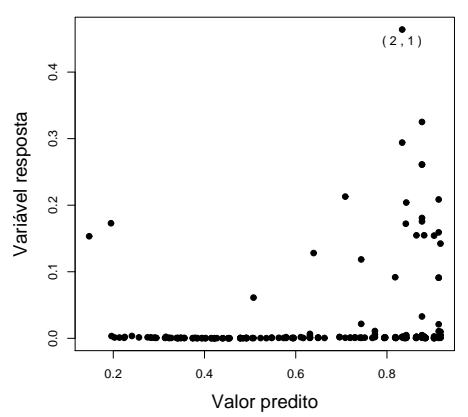

(b)

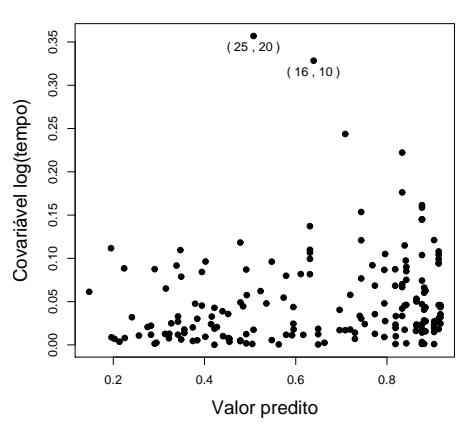

(c)

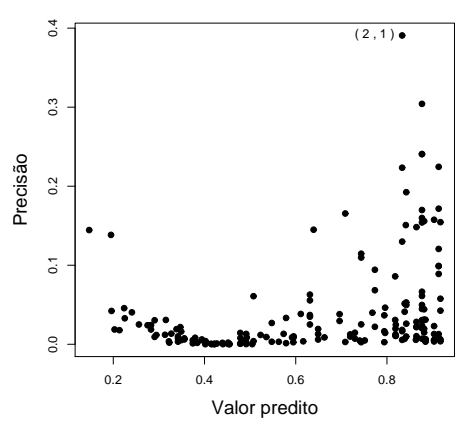

(d)

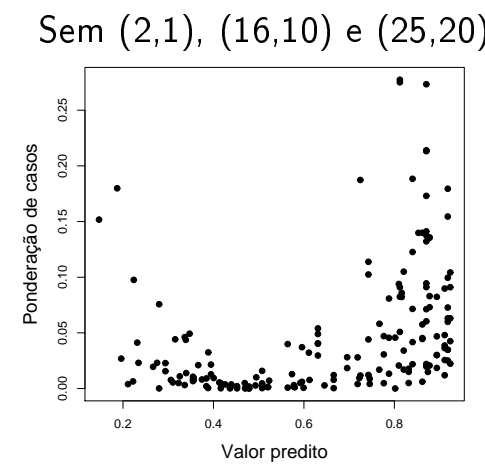

(e)

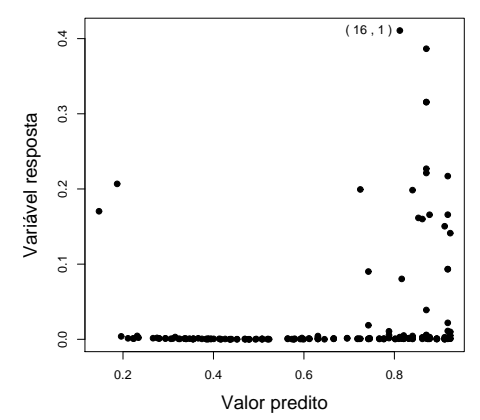

(f)

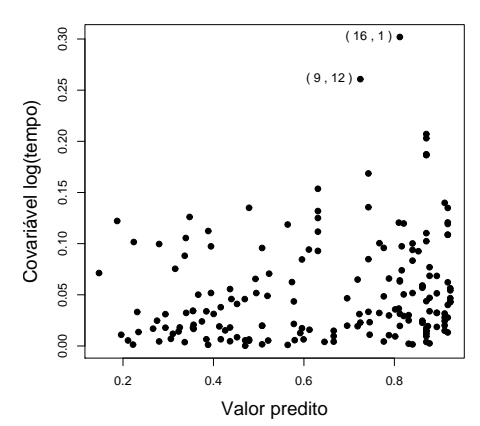

(g)

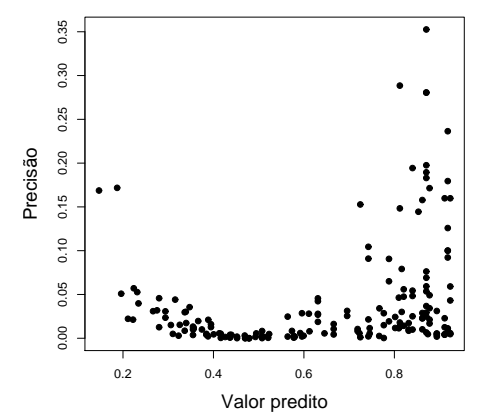

(h)

Figura 8.3: Gráficos de influência local para $\boldsymbol{\beta}$ considerando todas as observações (a)-(d) e sem as observações $(2,1),(16,10)$ e $(25,20)$ (e)-(h), para modelo de regressão beta sob homogeneidade da dispersão. Estudo de oftalmologia. 
Tabela 8.2: Estimativas e erros padrões dos parâmetros do modelo de regressão beta sob heterogeneidade da dispersão e com estrutura AR-1. Estudo de oftalmologia.

\begin{tabular}{cccccr} 
& & & \multicolumn{2}{c}{ Erro Padrão } & \multicolumn{1}{c}{ Nível } \\
\cline { 3 - 5 } Parâmetro & Estimativa & Robusto & Naive & Descritivo \\
\hline$\beta_{0}$ & Intercepto & 1,1957 & 0,1930 & 0,0316 & $<0,001$ \\
$\beta_{1}$ & $\log \left(\mathrm{x}_{1}\right)$ & 0,0770 & 0,4340 & 0,0762 & 0,859 \\
$\beta_{2}$ & $\log ^{2}\left(\mathrm{x}_{1}\right)$ & $-0,2131$ & 0,1486 & 0,0162 & 0,152 \\
$\beta_{3}$ & $\mathrm{x}_{2}$ & 0,4467 & 0,2778 & 0,0433 & 0,108 \\
$\gamma_{0}$ & Intercepto & 3,4649 & 0,0623 & 0,0104 & $<0,001$ \\
$\gamma_{1}$ & $\mathrm{x}_{2}$ & $-1,2307$ & 0,0556 & 0,0084 & $<0,001$ \\
$\alpha$ & Correlação & 0,3126 & & & \\
\hline
\end{tabular}

modela a precisão como diferente da que modela a média. De forma geral, concluímos que os pontos $(2,1),(16,10)$ e $(25,20)$ se destacam como pontos influentes também no modelo sob heterogeneidade da dispersão. Entretanto, esse ajuste deixa a medida de influência sob os esquemas de ponderação de casos e da variável resposta com pontos mais homogeneamente distribuídos entre os valores preditos, indicando ser mais homogênea a influência da variabilidade das observações sob as estimativas dos parâmetros de regressão (comparar Figura 8.3a e Figura 8.5d).

Excluindo os pontos $(2,1),(16,10)$ e $(25,20)$ da modelagem conjunta da média e da precisão, a Figura 8.6a mostra boas curvas ajustadas aos dados. As conclusões obtidas para os parâmetros $\gamma$ não se alteram quando comparadas com as obtidas com todas as observações no ajuste (Tabela 8.2), ou seja, os parâmetros continuam significantes. Com relação aos parâmetros de regressão envolvidos na modelagem da média, as conclusões são diferentes para $\beta_{2}$ e $\beta_{3}$ (que representam respectivamente as variáveis $\log ^{2}\left(\mathrm{x}_{1}\right)$ e $\mathrm{x}_{2}$ ), ou seja, rejeitamos a hipótese de que cada um desses parâmetros é igual a zero (com os respectivos níveis descritivos, $p=0,001$ e $p=0,023$ ), ao nível de $5 \%$ de significância. As variações percentuais nas estimativas desses dois parâmetros devido à exclusão das três observações causam um decréscimo de $57,6 \%$ na estimativa de $\beta_{2}$ e um acréscimo de $6,1 \%$ na de $\beta_{3}$. Os gráficos de diagnóstico apresentados na Figura 8.6 e na Figura 8.7 destacam as observações $(16,1)$ e $(9,12)$ como possíveis pontos influentes, entretanto, ao excluí-los da análise temos as mesmas conclusões do ajuste realizado apenas sem as observações $(2,1),(16,10)$ e $(25,20)$.

Em suma, se o interesse for apenas saber se a variável tempo após a cirurgia e a 


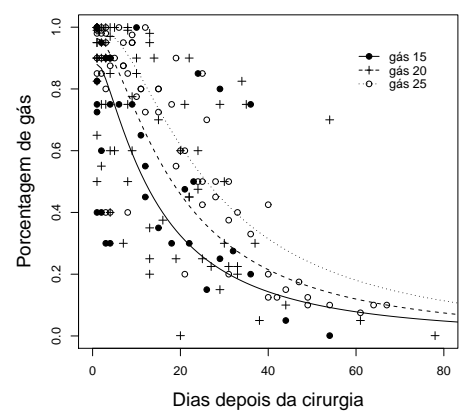

(a)

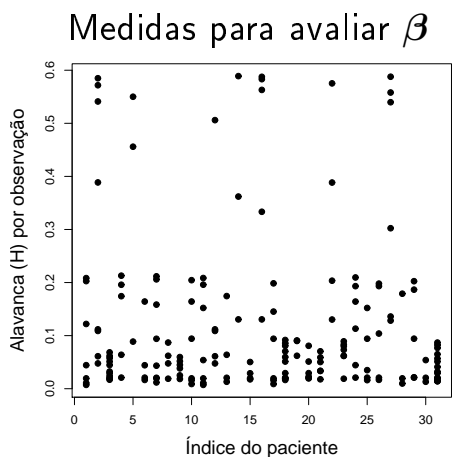

(b)

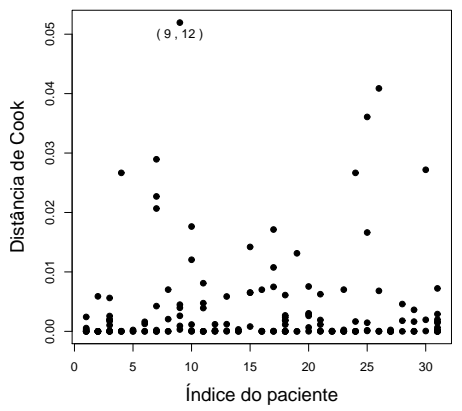

(c)

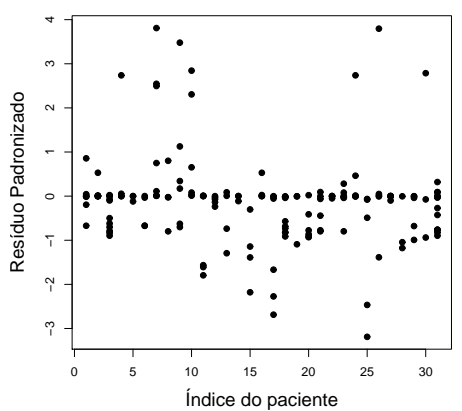

(d)

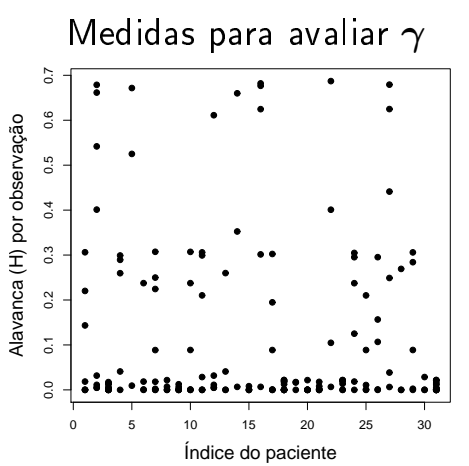

(e)

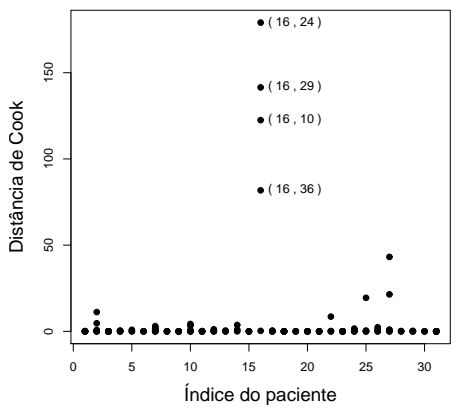

(f)

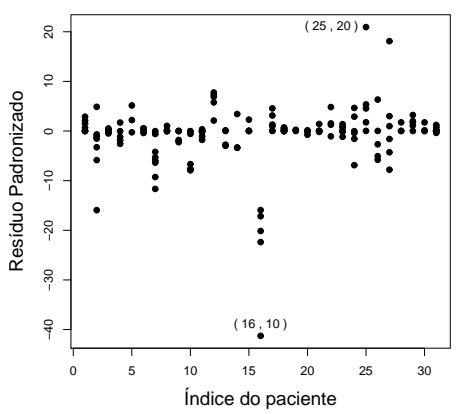

(g)

Figura 8.4: Curvas ajustadas (a); gráficos de diagnóstico do ajuste de $\boldsymbol{\beta}$ (b)-(d) e do ajuste de $\gamma(\mathrm{e})-(\mathrm{g})$, para modelos de regressão beta sob heterogeneidade da dispersão e com todas as observações. Estudo de oftalmologia. 


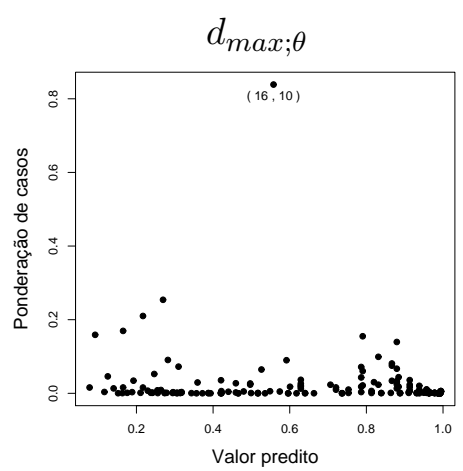

(a)

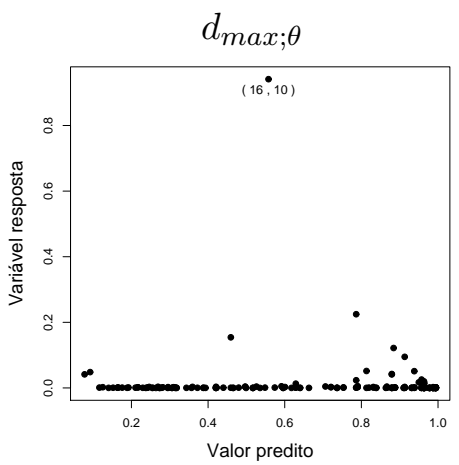

(b)

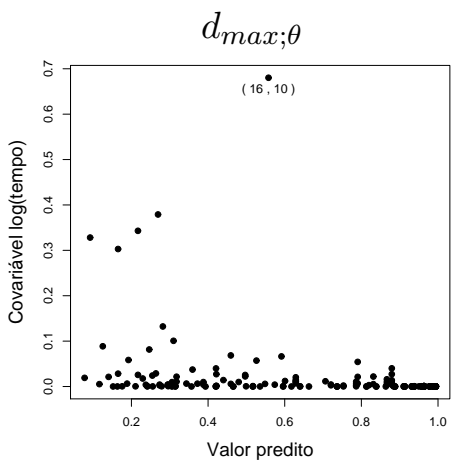

(c)

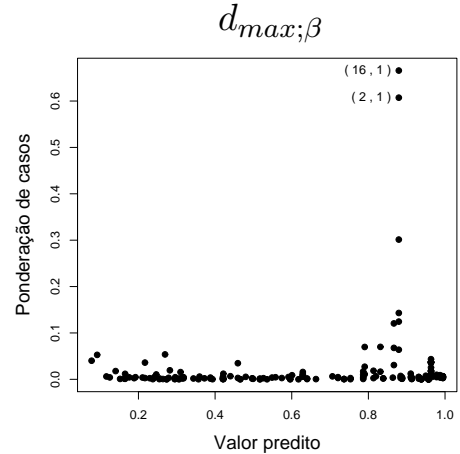

(d)

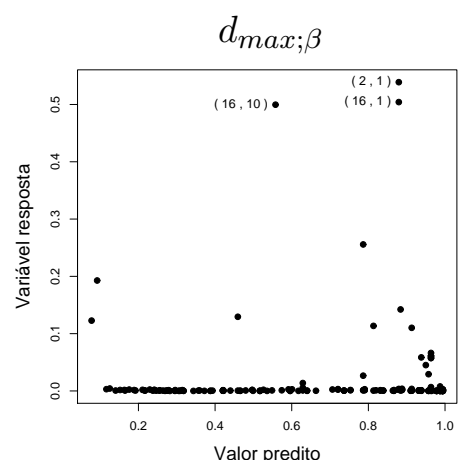

(e)

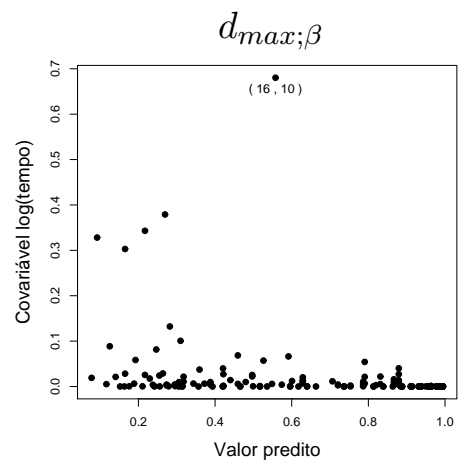

(f)

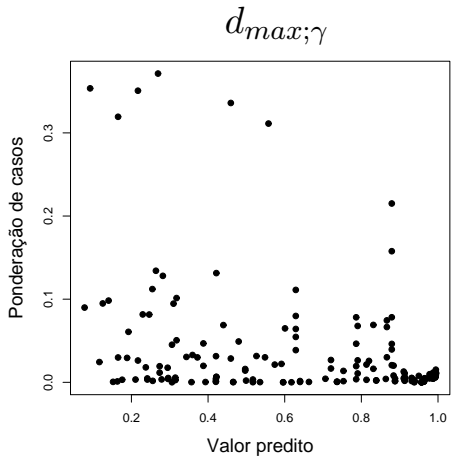

(g)

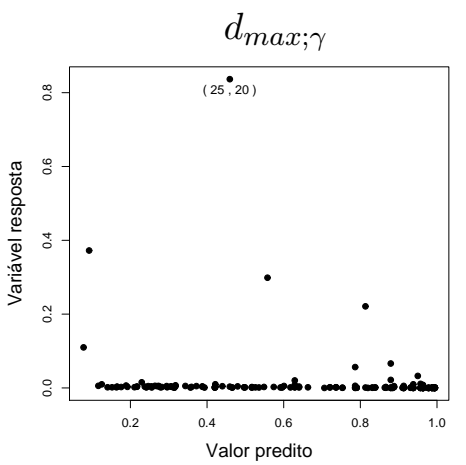

(h)

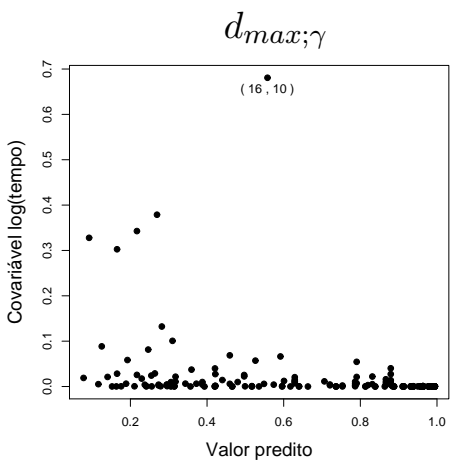

(i)

Figura 8.5: Gráficos de influência local para $\boldsymbol{\theta}$ (a)-(c); para $\boldsymbol{\beta}$ (d)-(f) e para $\boldsymbol{\gamma}(\mathrm{g})$-(i), para modelos de regressão beta sob heterogeneidade da dispersão e com todas as observações. Estudo de oftalmologia. 
concentração de gás são importantes ou não para explicar a porcentagem de volume de gás ainda presente nos olhos dos pacientes com relação ao volume injetado no momento da cirurgia, as conclusões obtidas para o ajuste da média sob homogeneidade da dispersão e para o ajuste da média sob a modelagem da precisão, ambos sem os pontos $(2,1),(16,10)$ e $(25,20)$, são as mesmas ao nível de $5 \%$ de significância.

Contudo, se o interesse for algum que utilize as estimativas obtidas aos parâmetros de regressão, como por exemplo, fazer previsão, então indicamos utilizar o ajuste obtido com a modelagem conjunta da média e da precisão e sem as observações $(2,1),(16,10)$ e $(25,20)$.

\section{SIMPLEX}

Conforme visto na Parte I deste trabalho, a distribuição simplex também é adequada para modelar dados pertencentes ao intervalo $(0,1)$. Dessa forma, aplicamos uma nova modelagem aos dados do estudo oftalmológico utilizando as equações de estimação descritas no Capítulo 3.

Nesse caso, ajustamos um modelo com medidas repetidas para variável resposta considerando distribuição simplex, função de ligação logito e homogeneidade da dispersão. Assim, a modelagem da média foi dada por

$$
\log \left(\frac{\mu_{i j}}{1-\mu_{i j}}\right)=\beta_{0}+\beta_{1} \log \left(\mathrm{x}_{1 i j}\right)+\beta_{2} \log ^{2}\left(\mathrm{x}_{1 i j}\right)+\beta_{3}\left(\mathrm{x}_{2 i j}\right),
$$

com $\mathrm{x}_{1 i j}$ e $\mathrm{x}_{2 i j}$ descritos em (8.1).

Pela medida $Q I C$, o ajuste com a estrutura AR-1 foi escolhido como o mais adequado para explicar a correlação entre as respostas da mesma unidade experimental. As estimativas dos parâmetros desse modelo estão apresentadas na Tabela 8.3 e as curvas ajustadas, na Figura 8.8a. A mesma conclusão dada à Tabela 8.1 também se aplica à Tabela 8.3, em que apenas o coeficiente $\beta_{2}$ é significante para descrever a queda do volume de gás ainda presente nos olhos dos pacientes (nível descritivo, $p<0,001$ ).

As Figuras 8.8b-d apresentam a medida de alavanca baseada na matriz de projeção, a distância de Cook e o resíduo padronizado, respectivamente. Para essas figuras, temos que a distância de Cook e o resíduo padrozinado do paciente $16 \mathrm{com}$ dez dias após a cirurgia, $(16,10)$, e do paciente 25 com vinte dias após a cirurgia , $(25,20)$, destacam-se das demais observações, indicando serem possíveis pontos influentes e/ou aberrantes.

Os gráficos de influência local para os seguintes esquemas de perturbação: ponderação de casos, da variável resposta, da covariável logaritmo neperiano do tempo $\left(\log \left(\mathrm{x}_{1}\right)\right)$ e da precisão, quando consideramos todas as observações, são apresentados, respectivamente, 


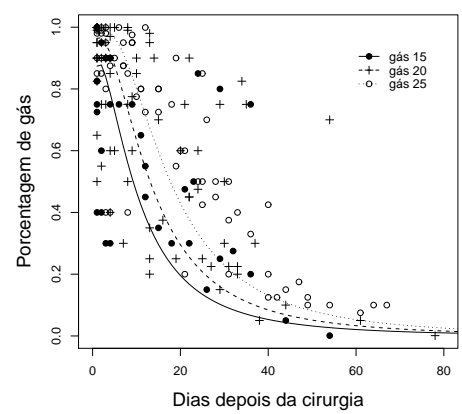

(a)

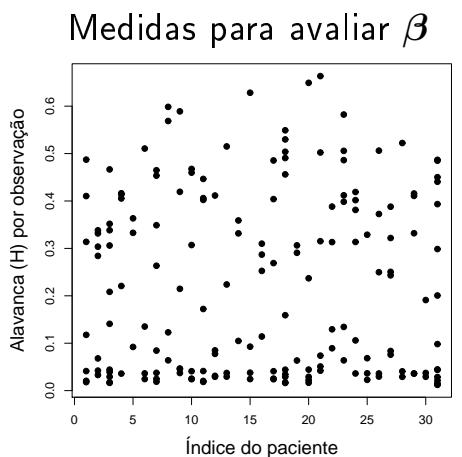

(b)

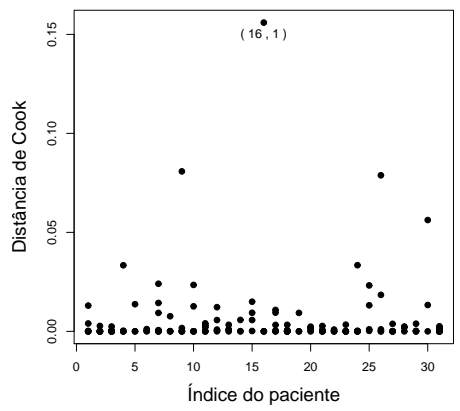

(c)

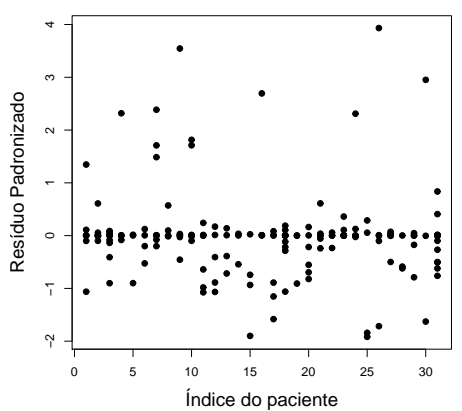

(d)

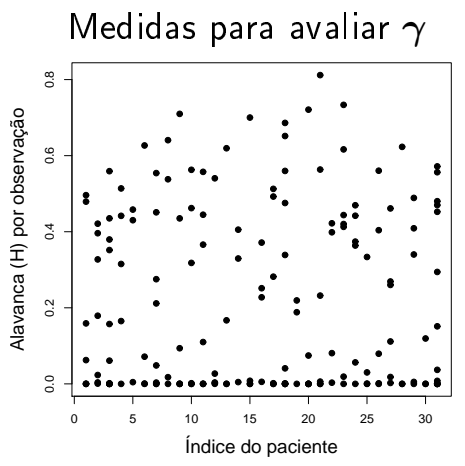

(e)

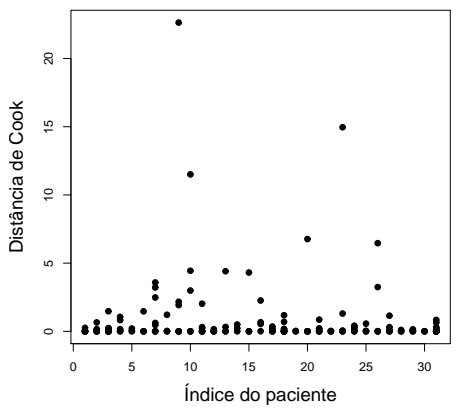

(f)

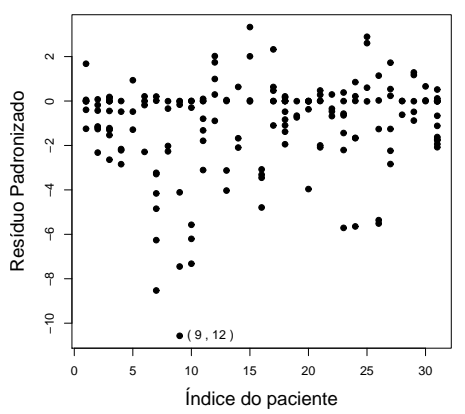

(g)

Figura 8.6: Curvas ajustadas (a); gráficos de diagnóstico do ajuste de $\boldsymbol{\beta}$ (b)-(d) e do ajuste de $\boldsymbol{\gamma}$ (e)-(g), para modelos de regressão beta sob heterogeneidade da dispersão e sem as observações $(2,1),(16,10)$ e $(25,20)$. Estudo de oftalmologia. 


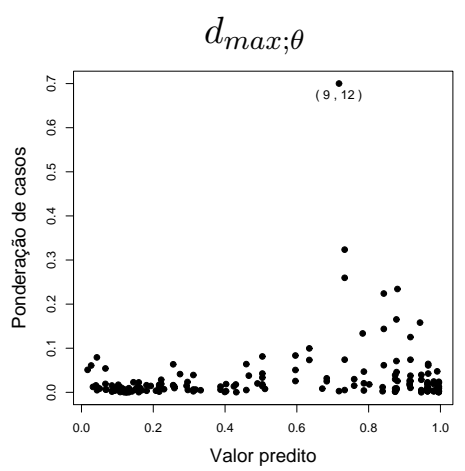

(a)

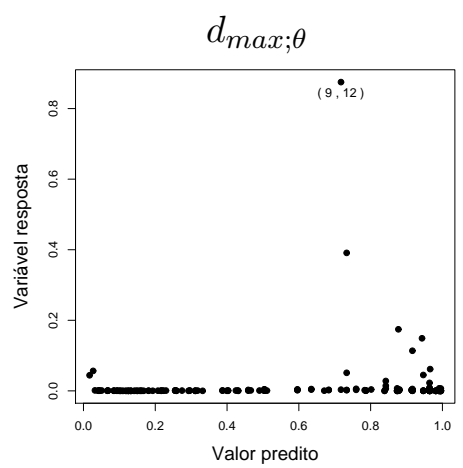

(b)

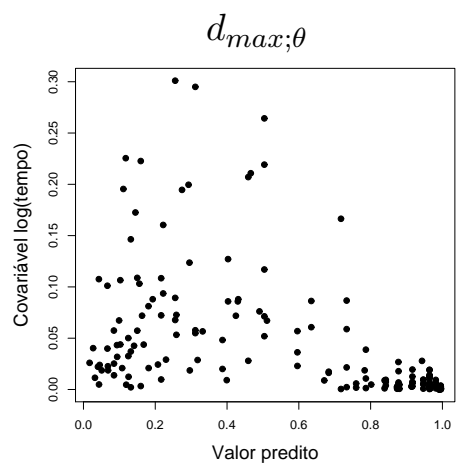

(c)

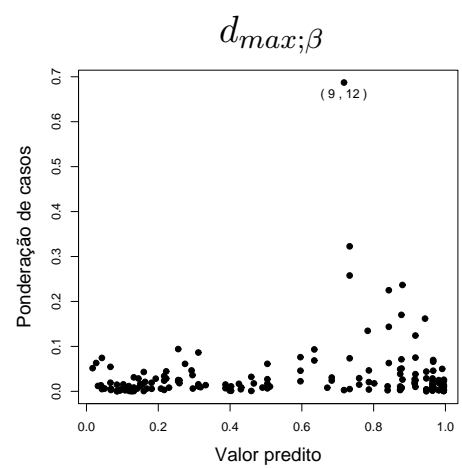

(d)

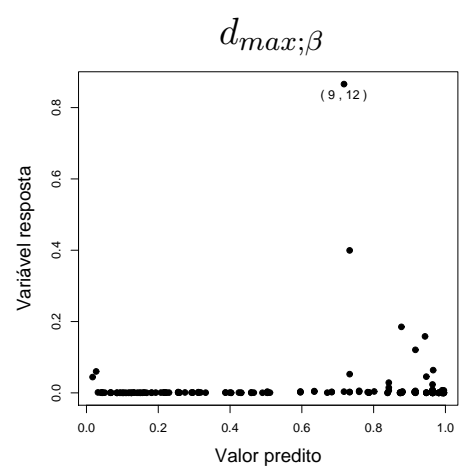

(e)

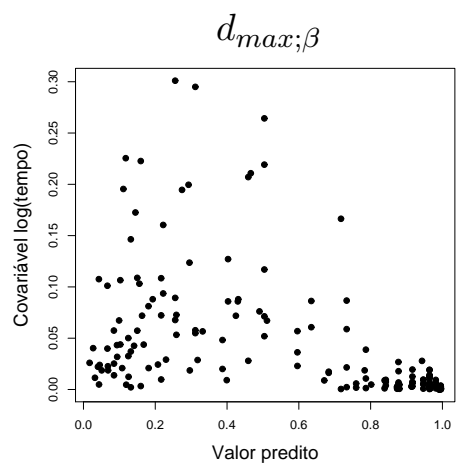

(f)

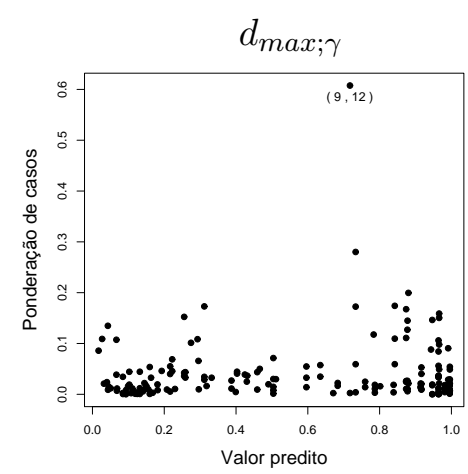

(g)

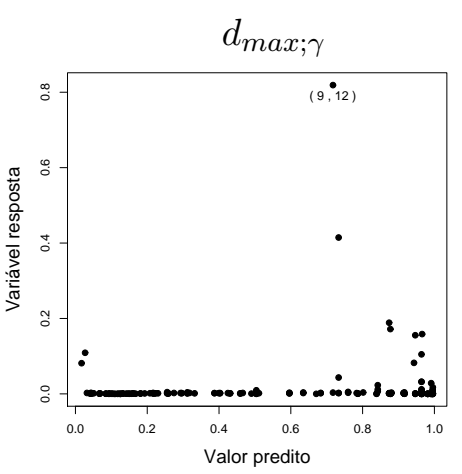

(h)

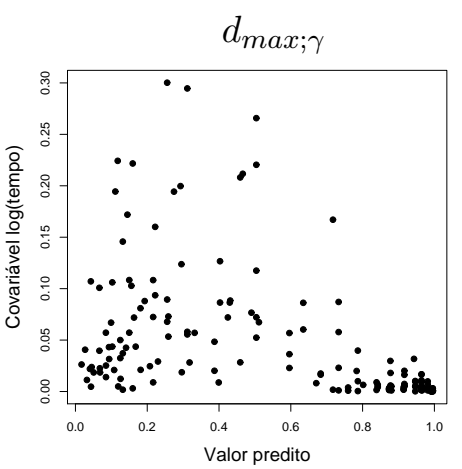

(i)

Figura 8.7: Gráficos de influência local para $\boldsymbol{\theta}$ (a)-(c); para $\boldsymbol{\beta}(\mathrm{d})-(\mathrm{f})$ e para $\boldsymbol{\gamma}(\mathrm{g})$-(i), para modelos de regressão beta sob heterogeneidade da dispersão e sem as observações $(2,1),(16,10)$ e $(25,20)$. Estudo de oftalmologia. 
Tabela 8.3: Estimativas e erros padrões dos parâmetros do modelo de regressão simplex sob homogeneidade da dispersão e com estrutura AR-1. Estudo de oftalmologia.

\begin{tabular}{lccccr}
\hline & & & \multicolumn{2}{c}{ Erro Padrão } & \multicolumn{1}{c}{ Nível } \\
\multicolumn{2}{c}{ Parâmetro } & Estimativa & Robusto & Naive & Descritivo \\
\hline$\beta_{0}$ & Intercepto & 2,7341 & 0,2964 & 0,3558 & $<0,001$ \\
$\beta_{1}$ & $\log \left(\mathrm{x}_{1}\right)$ & 0,0977 & 0,1987 & 0,3460 & 0,623 \\
$\beta_{2}$ & $\log ^{2}\left(\mathrm{x}_{1}\right)$ & $-0,3501$ & 0,0493 & 0,0847 & $<0,001$ \\
$\beta_{3}$ & $\mathrm{x}_{2}$ & 0,3026 & 0,1777 & 0,1582 & 0,089 \\
$\phi^{-1}$ & Dispersão & 200,57 & & & \\
$\alpha$ & Correlação & 0,5112 & & & \\
\hline
\end{tabular}

nas Figuras 8.9a-d. Novamente, temos as observações $(16,10)$ e $(25,20)$ se destacando como influentes nos quatro esquemas de perturbação.

O ajuste do modelo $(8.3)$ sem as observações $(16,10)$ e $(25,20)$ mantém a significância do parâmetro $\beta_{2}$ e altera a conclusão para a covariável concentração de gás, já que $\beta_{3}$ passa a ser significativa (ambos níveis descritivos, $p<0,001$ ). Na Figura 8.8e, as três curvas ajustadas ficam mais espaçadas quando comparadas com as curvas da Figura 8.8a que contém todas as observações. Além disso, notamos que novos pontos se destacam como influentes e/ou aberrantes (Figuras 8.8g-h e Figuras 8.9e-h) e que observações com valores preditos superiores a 0,8 exercem maiores influências nas estimativas dos parâmetros de regressão $\boldsymbol{\beta}$ (ver Figura 8.9e e Figura 8.9h), sendo um indicativo de possível heterogeneidade do parâmetro de dispersão.

Para avaliarmos essa possível presença de heterogeneidade da dispersão, fizemos uma modelagem conjunta da média sob o modelo (8.3) e da dispersão sob o modelo:

$$
\log \left(\phi_{i j}^{-1}\right)=\gamma_{0}+\gamma_{1}\left(\mathrm{x}_{2 i j}\right)
$$

Para esse ajuste, as estimativas estão na Tabela 8.4 e as curvas ajustadas, na Figura 8.10a. Essa tabela nos mostra que o modelo heteroscedástico, representado pelo parâmetro $\gamma_{1}$, não é significante. As Figuras 8.10b-g e Figura 8.11, que apresentam algumas medidas de diagnóstico, destacam os pontos $(9,12),(16,10)$ e $(25,20)$ como possíveis pontos influentes e/ou aberrantes.

Apesar do modelo (8.4) não ter sido significante com todas as observações, foi novamente ajustado sem as observações $(9,12),(16,10)$ e $(25,20)$, com intuito de detectar uma possível significância de $\gamma_{1}$ e a correção da maior influência nas estimativas dos parâmetros 


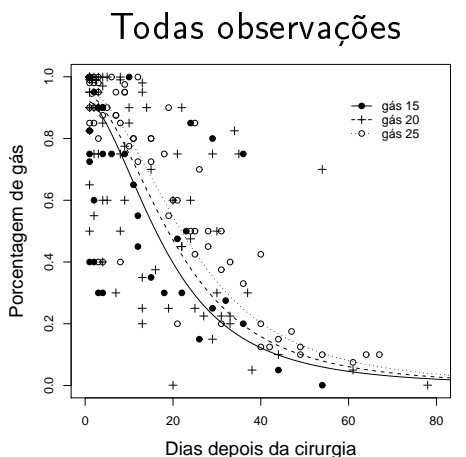

(a)

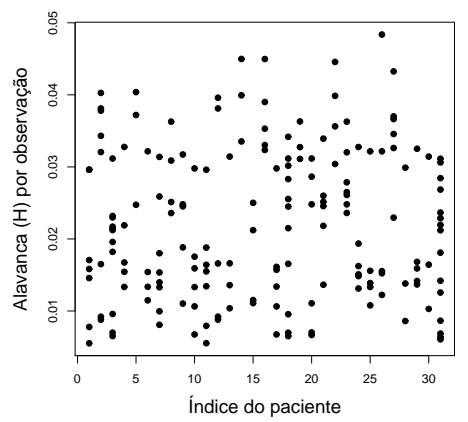

(b)

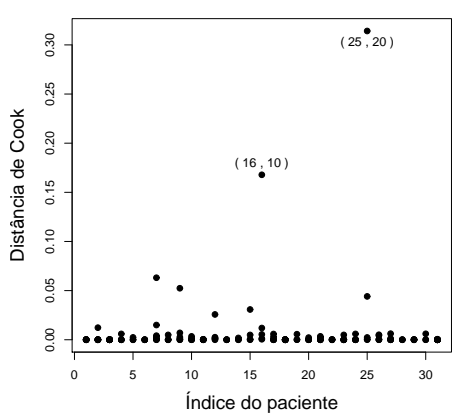

(c)

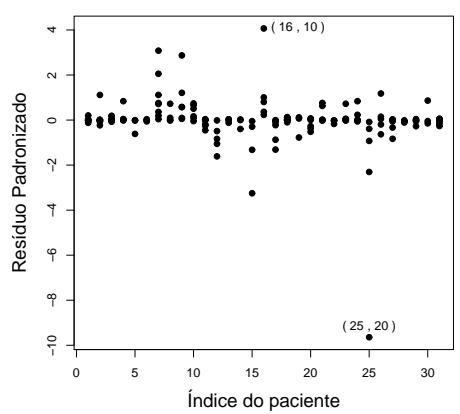

(d)

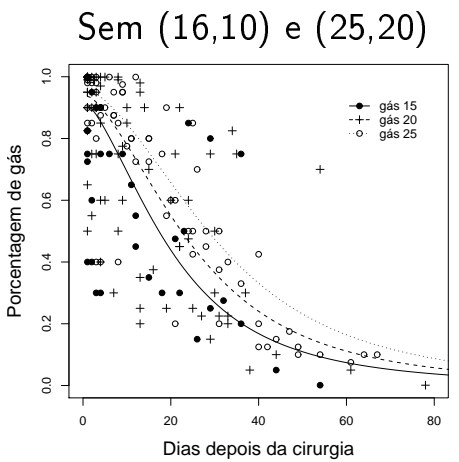

(e)

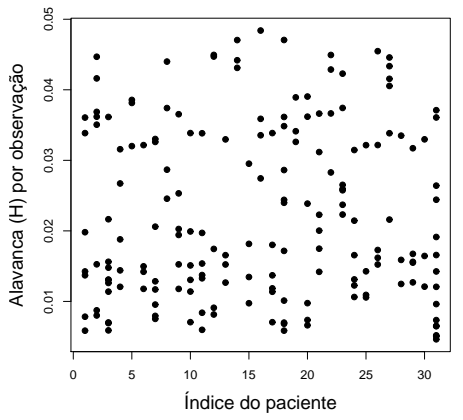

(f)

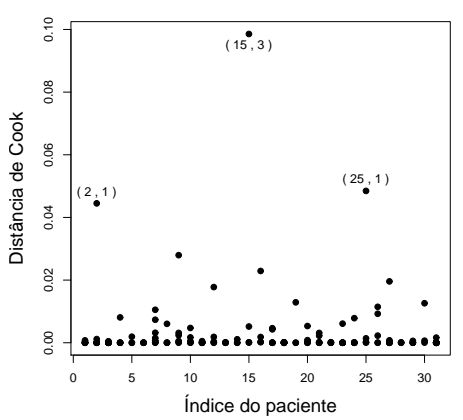

(g)

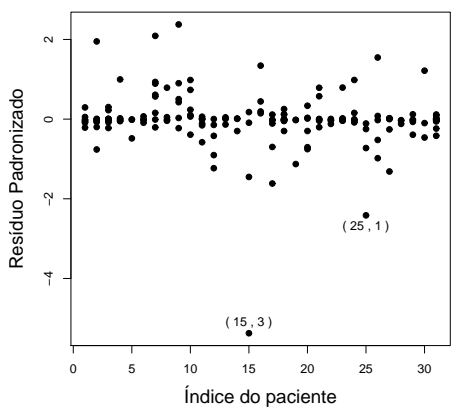

(h)

Figura 8.8: Curvas ajustadas e gráficos de diagnóstico com todas as observações (a)-(d) e sem as observações $(16,10)$ e $(25,20)$ (e)-(h), para modelo de regressão simplex sob homogeneidade da dispersão. Estudo de oftalmologia. 


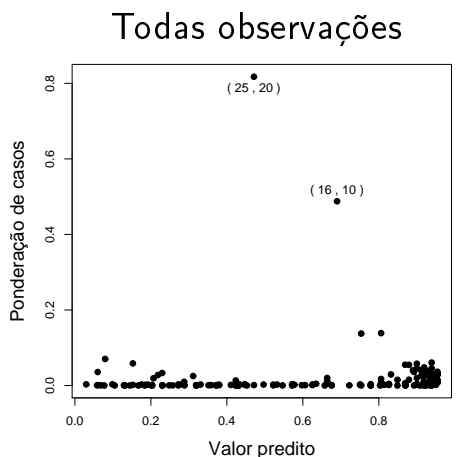

(a)

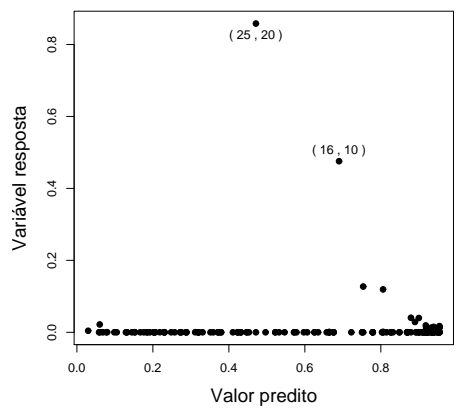

(b)

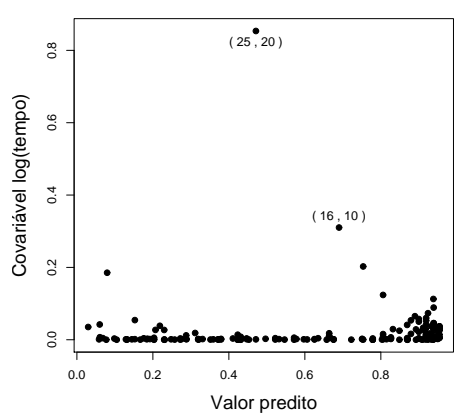

(c)

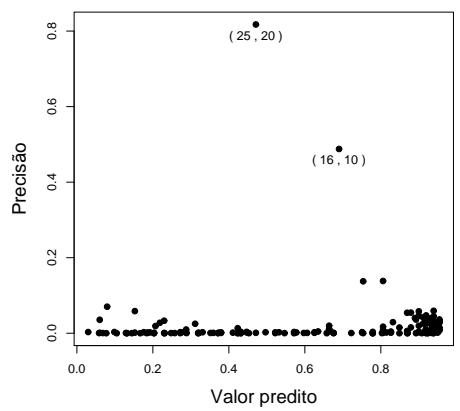

(d)

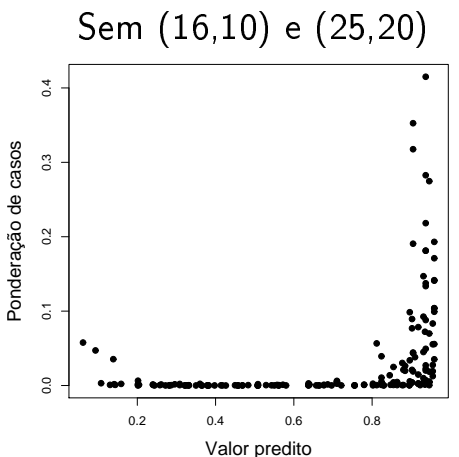

(e)

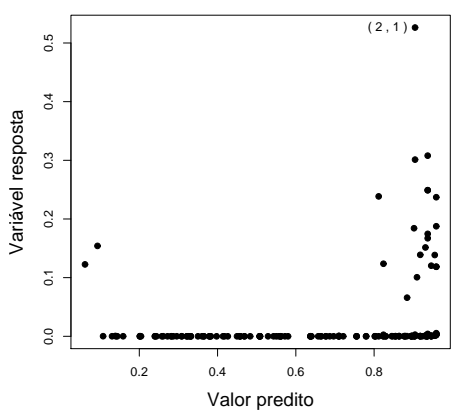

(f)

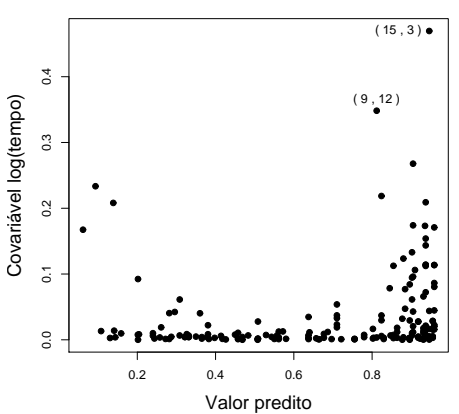

(g)

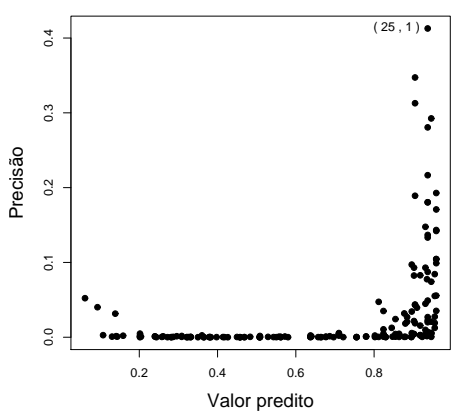

(h)

Figura 8.9: Gráficos de influência local para $\boldsymbol{\beta}$ considerando todas as observações (a)-(d) e sem as observações $(16,10)$ e $(25,20)$ (e)-(h), para modelo de regressão simplex sob homogeneidade da dispersão. Estudo de oftalmologia. 
de regressão causada pelas observações com valores preditos superiores a 0,8. Entretanto, a exclusão dessas três observações não alterou a conclusão de nenhum teste apresentado na Tabela 8.4 (ajuste com todas as observações), nem corrigiu a influência nas estimativas de $\boldsymbol{\beta}$ causada pelas observações com valores preditos superiores a 0,8 (comparar Figura 8.9e e Figura 8.13d).

Tabela 8.4: Estimativas e erros padrões dos parâmetros do modelo de regressão simplex sob heterogeneidade da dispersão e com estrutura AR-1. Estudo de oftalmologia.

\begin{tabular}{rccccr}
\hline & & \multicolumn{2}{c}{ Erro Padrão } & \multicolumn{1}{c}{ Nível } \\
\cline { 3 - 5 } & Parâmetro & Estimativa & Robusto & Naive & Descritivo \\
\hline$\beta_{0}$ & Intercepto & 2,7451 & 0,2880 & 0,3527 & $<0,001$ \\
$\beta_{1}$ & $\log \left(\mathrm{x}_{1}\right)$ & 0,0735 & 0,1950 & 0,3429 & 0,706 \\
$\beta_{2}$ & $\log ^{2}\left(\mathrm{x}_{1}\right)$ & $-0,3438$ & 0,0514 & 0,0840 & $<0,001$ \\
$\beta_{3}$ & $\mathrm{x}_{2}$ & 0,3431 & 0,1727 & 0,1582 & 0,047 \\
$\gamma_{0}$ & Intercepto & 5,3151 & 0,2362 & 0,1095 & $<0,001$ \\
$\gamma_{1}$ & $\mathrm{x}_{2}$ & $-0,2212$ & 0,3125 & 0,1424 & 0,479 \\
$\alpha$ & Correlação & 0,5177 & & & \\
\hline
\end{tabular}

De maneira geral, podemos concluir que nenhum dos modelos realizados com a distribuição simplex apresentou diagnóstico satisfatório que fizesse escolher um como mais adequado para ajustar os dados.

\subsection{Aplicação II - Estudo de desenvolvimento motor de bebês}

Um estudo apresentado em Botter et al. (2006) tem como objetivo comparar as performances motoras entre recém-nascidos Pré-termo (nascidos até 36 semanas de gestação) e $A$ termo (nascidos após 36 semanas de gestação).

No acompanhamento do desenvolvimento dos bebês, foi levada em conta a idade corrigida (em dias), que corresponde à diferença entre a idade cronológica (tempo de vida desde o nascimento até o momento presente) e o índice de prematuridade (número de semanas que faltaram entre o nascimento prematuro e as 40 semanas de idade gestacional).

Os recém-nascidos foram observados de 2 a 13 vezes, medindo-se a quantidade de 


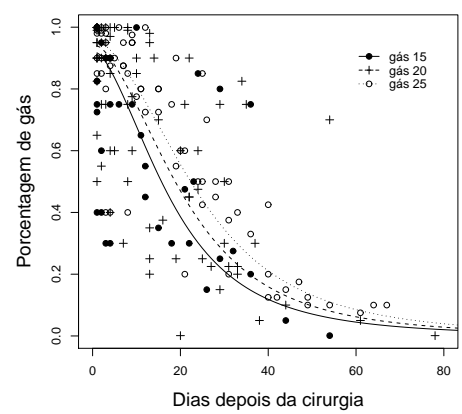

(a)

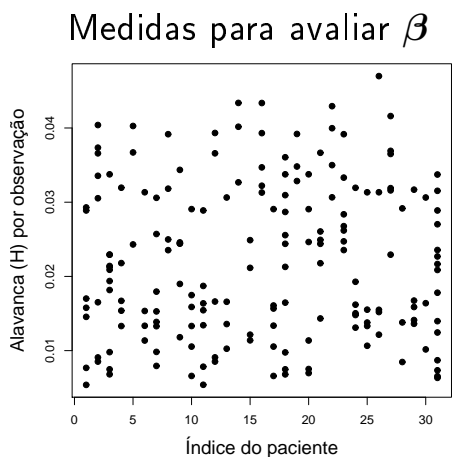

(b)

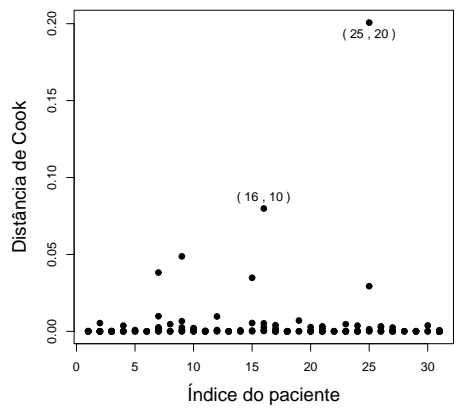

(c)

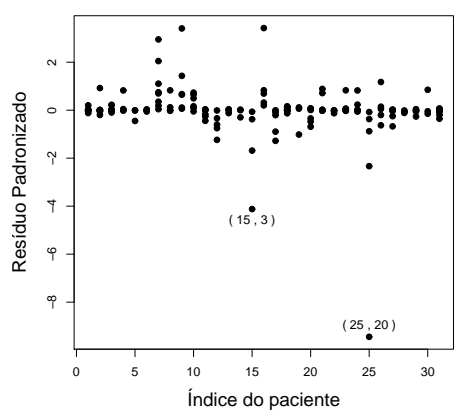

(d)

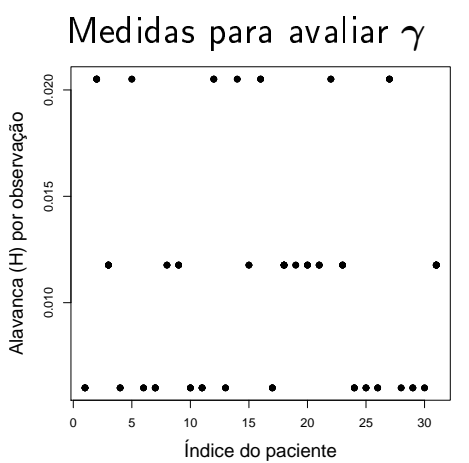

(e)

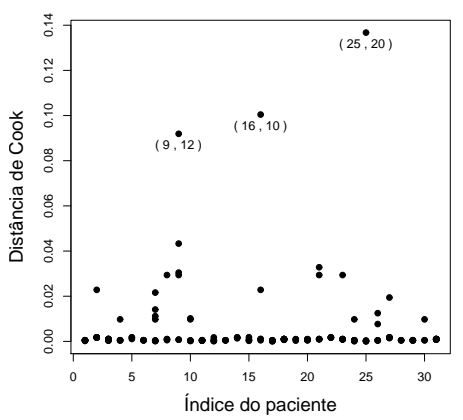

(f)

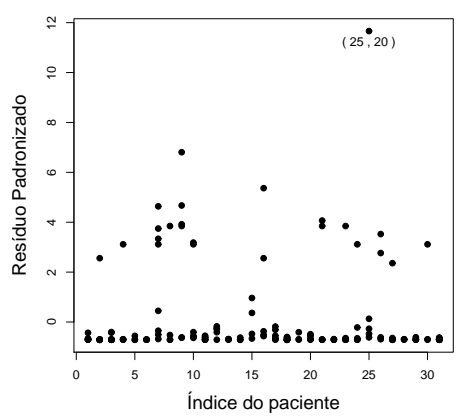

(g)

Figura 8.10: Curvas ajustadas (a); gráficos de diagnóstico do ajuste de $\boldsymbol{\beta}$ (b)-(d) e do ajuste de $\gamma(\mathrm{e})-(\mathrm{g})$, para modelos de regressão simplex sob heterogeneidade da dispersão e com todas as observações. Estudo de oftalmologia. 


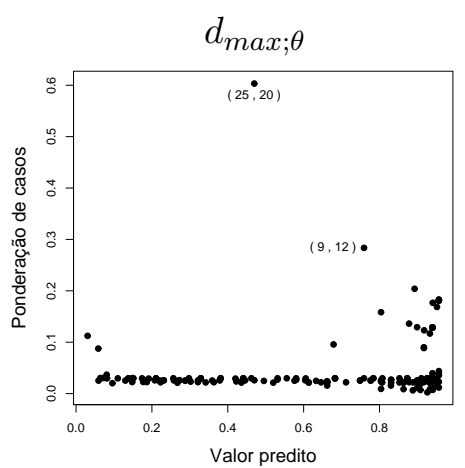

(a)

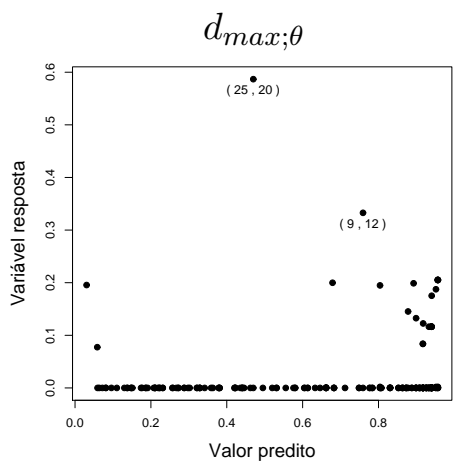

(b)

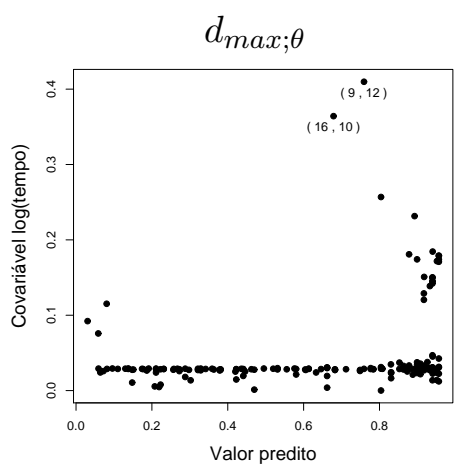

(c)

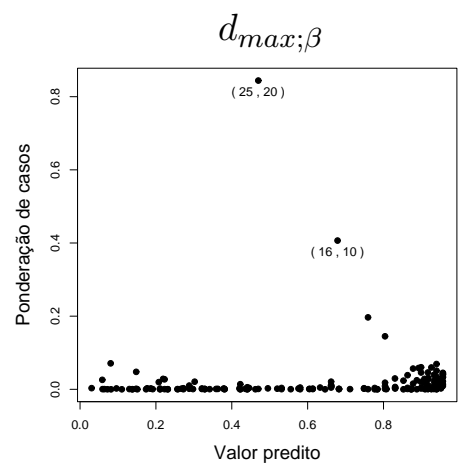

(d)

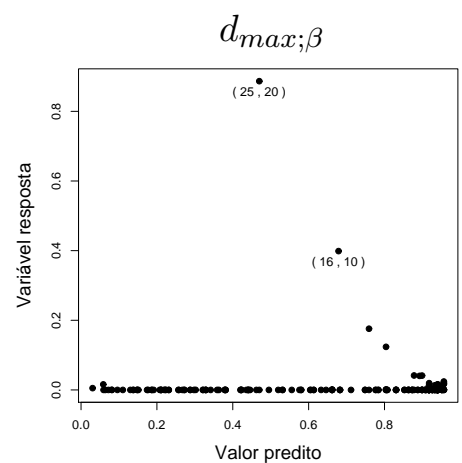

(e)

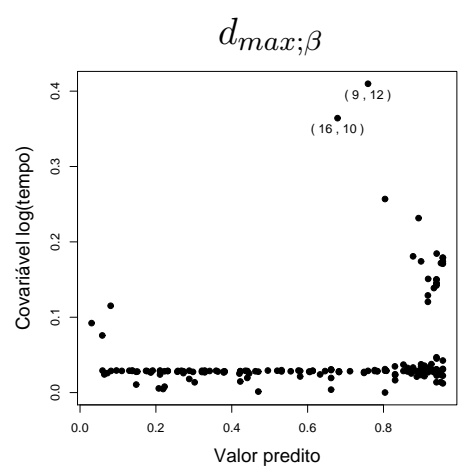

(f)

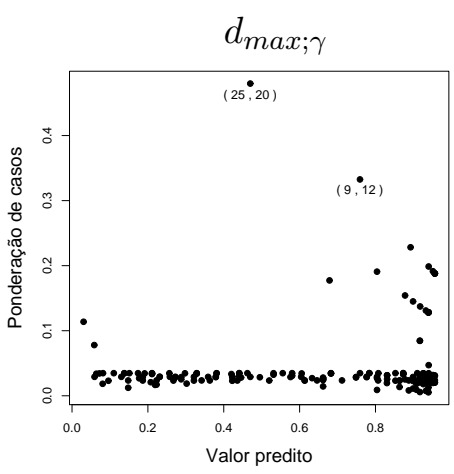

(g)

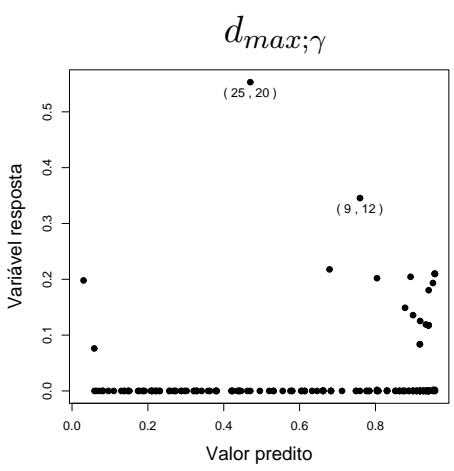

(h)

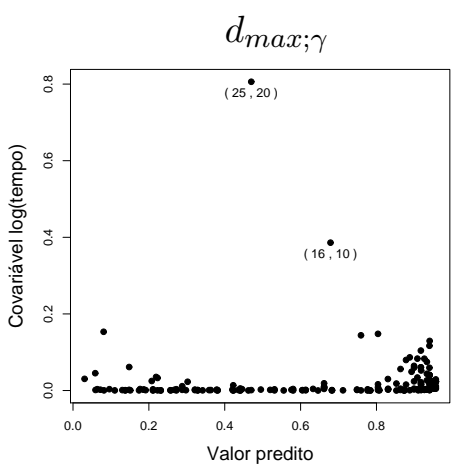

(i)

Figura 8.11: Gráficos de influência local para $\boldsymbol{\theta}$ (a)-(c); para $\boldsymbol{\beta}(\mathrm{d})$-(f) e para $\boldsymbol{\gamma}(\mathrm{g})$-(i), para modelos de regressão simplex sob heterogeneidade da dispersão e com todas as observações. Estudo de oftalmologia. 


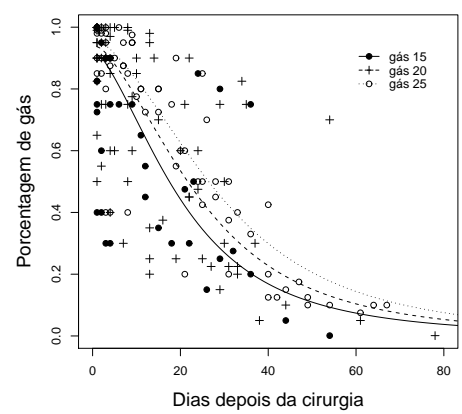

(a)

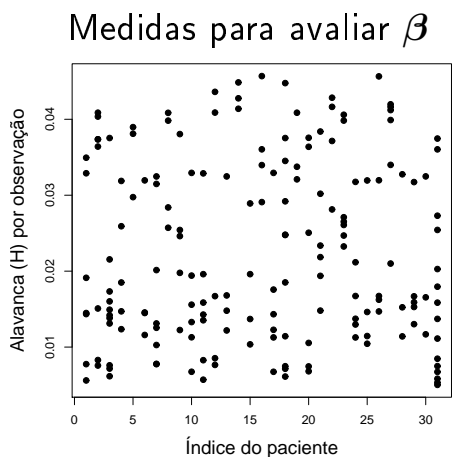

(b)

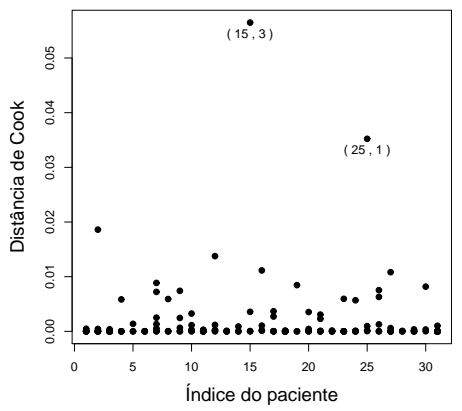

(c)

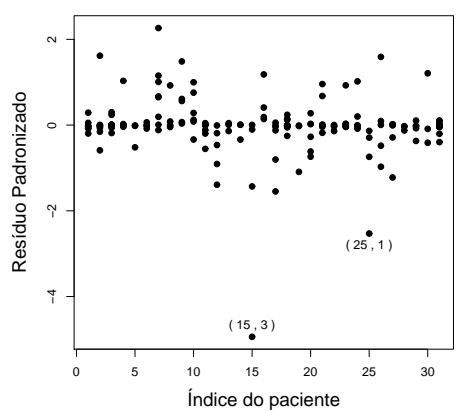

(d)

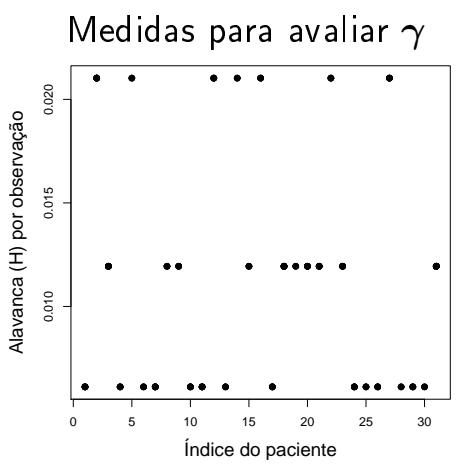

(e)

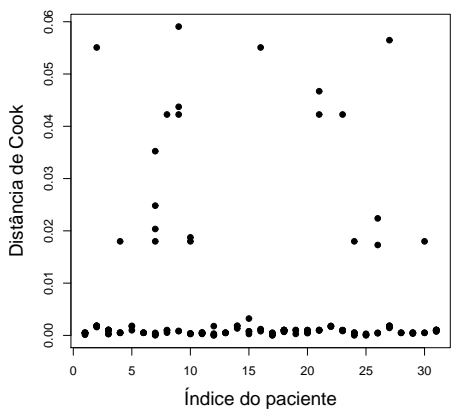

(f)

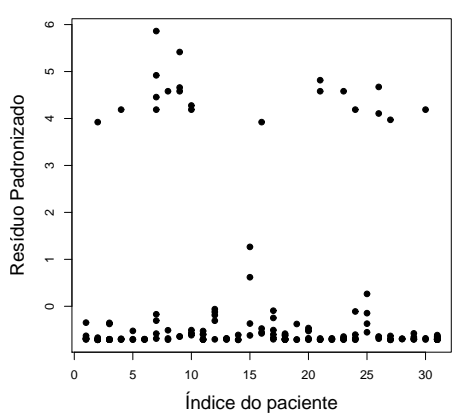

(g)

Figura 8.12: Curvas ajustadas (a); gráficos de diagnóstico do ajuste de $\boldsymbol{\beta}$ (b)-(d) e do ajuste de $\boldsymbol{\gamma}$ (e)-(g), para modelos de regressão simplex sob heterogeneidade da dispersão e sem as observações $(9,12),(16,10)$ e $(25,20)$. Estudo de oftalmologia. 


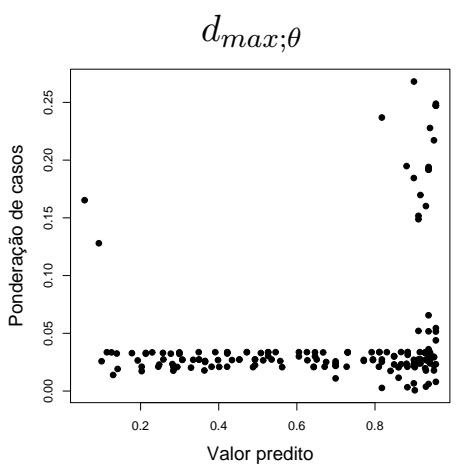

(a)

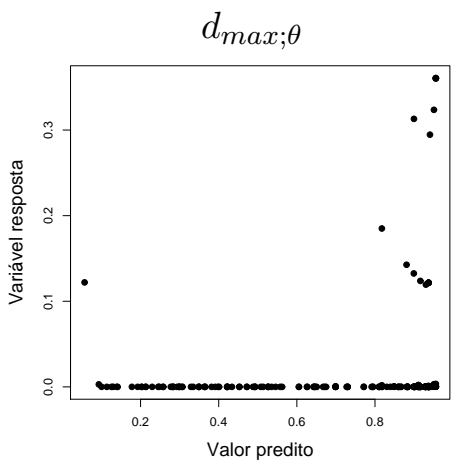

(b)

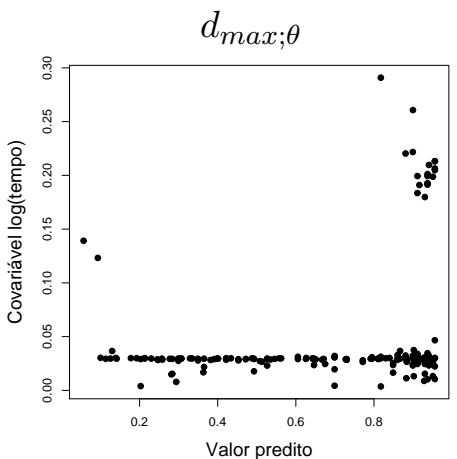

(c)

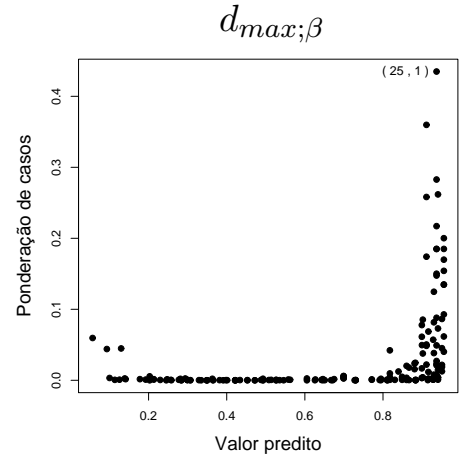

(d)

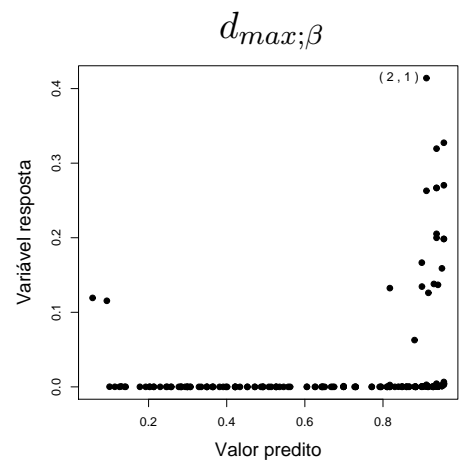

(e)

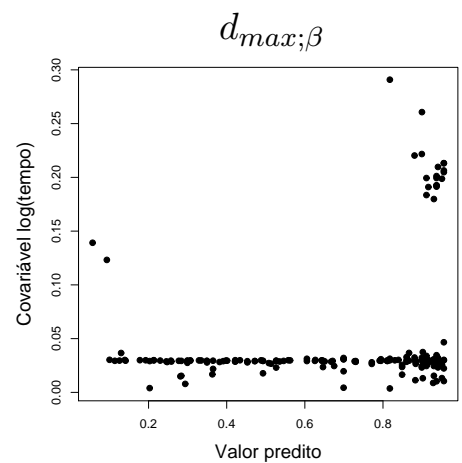

(f)

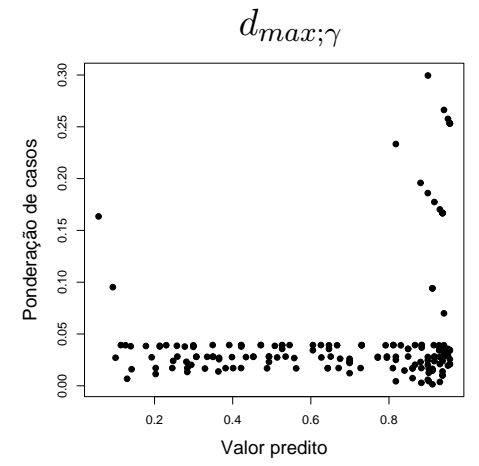

(g)

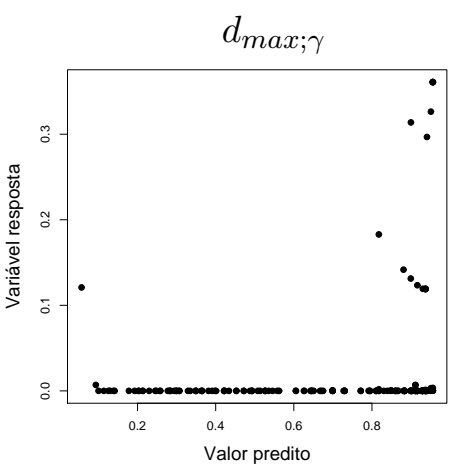

(h)

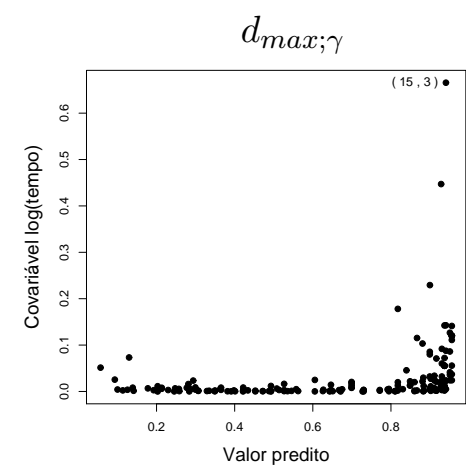

(i)

Figura 8.13: Gráficos de influência local para $\boldsymbol{\theta}$ (a)-(c); para $\boldsymbol{\beta}(\mathrm{d})$-(f) e para $\boldsymbol{\gamma}(\mathrm{g})$-(i), para modelos de regressão simplex sob heterogeneidade da dispersão e sem as observações $(9,12)$, $(16,10)$ e $(25,20)$. Estudo de oftalmologia. 


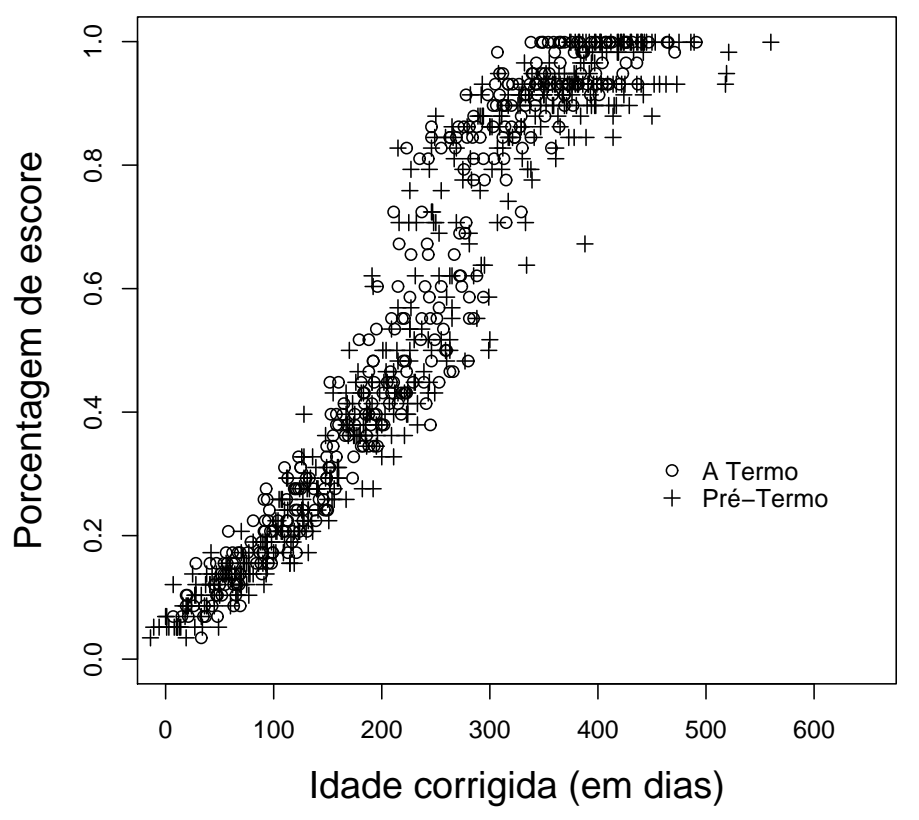

Figura 8.14: Gráfico de dispersão da porcentagem de escore versus idade corrigida, para os grupos A termo e Pré-termo. Estudo de desenvolvimento motor de bebês.

posições (escore) que o bebê era capaz de realizar (pode variar de 0 a 58) e a idade em que ocorreu a aquisição das posições. A variável resposta foi definida como a porcentagem de escore atingido com relação à pontuação máxima (58 pontos) ${ }^{2}$. A Figura 8.14 apresenta o comportamento dessa variável resposta para os dois grupos avaliados.

\section{BETA}

Para esses dados, ajustamos um modelo com medidas repetidas considerando distribuição beta, função de ligação complemento log-log, homogeneidade da dispersão e estrutura de correlação AR-1. A modelagem da média foi dada por

$$
\log \left[-\log \left(1-\mu_{i j}\right)\right]=\beta_{0}+\beta_{1}\left(\mathrm{x}_{1 i j}\right)+\beta_{2}\left(\mathrm{x}_{2 i j}\right)+\beta_{3}\left(\mathrm{x}_{1 i j} * \mathrm{x}_{2 i j}\right)
$$

sendo $x_{1 i j}=\left\{\begin{array}{ll}0 & \text { se "A Termo" } \\ 1 & \text { se "Pré-Termo" }\end{array}\right.$ e $x_{2 i j}$ a idade corrigida (em dias).

As estimativas dos parâmetros desse modelo são apresentadas na Tabela 8.5 e as curvas ajustadas, na Figura 8.15a. Nessa tabela, observamos significância da interação

\footnotetext{
${ }^{2}$ Para valores da variável resposta iguais a 0 e 1 consideramos, respectivamente, iguais a 0,001 e 0,999.
} 
entre grupo $\left(\mathrm{x}_{1}\right)$ e tempo $\left(\mathrm{x}_{2}\right)$ para descrever o aumento médio do escore atingido com relação à pontuação máxima (nível descritivo, $p=0,001$ ).

Tabela 8.5: Estimativas e erros padrões dos parâmetros do modelo de regressão beta sob homogeneidade da dispersão e com estrutura AR-1. Estudo de desenvolvimento motor de bebês.

\begin{tabular}{cccccr}
\hline & & \multicolumn{3}{c}{ Erro Padrão } & \multicolumn{1}{c}{ Nível } \\
\multicolumn{2}{c}{ Parâmetro } & Estimativa & Robusto & Naive & Descritivo \\
\hline$\beta_{0}$ & Intercepto & $-2,1867$ & 0,0575 & 0,0507 & $<0,001$ \\
$\beta_{1}$ & $\mathrm{x}_{1}$ & 0,0650 & 0,0727 & 0,0658 & 0,372 \\
$\beta_{2}$ & $\mathrm{x}_{2}$ & 0,0087 & 0,0002 & 0,0002 & $<0,001$ \\
$\beta_{3}$ & $\mathrm{x}_{1} * \mathrm{x}_{2}$ & $-0,0007$ & 0,0002 & 0,0002 & 0,001 \\
$\phi$ & Precisão & 13,9250 & & & \\
$\alpha$ & Correlação & 0,2513 & & & \\
\hline
\end{tabular}

A medida de alavanca baseada na matriz de projeção, a distância de Cook e o resíduo padronizado estão apresentados nas Figuras 8.15b-d, respectivamente. Nesses gráficos, a observação referente ao bebê 30 com idade corrigida de 491 dias, (30,491), se destaca como possível ponto alavanca e a observação do bebê 106 avaliado com idade corrigida de 560 dias, $(106,560)$, como possível ponto alavanca e influente. Nas Figuras 8.15e-h, encontramos os gráficos de influência local, respectivamente, para os seguintes esquemas de perturbação: ponderação de casos, da variável resposta, da covariável idade corrigida $\left(\mathrm{x}_{2}\right)$ e da precisão. Nas Figuras 8.15e e 8.15h, podemos ter um indicativo de presença de heterogeneidade do parâmetro de precisão já que os valores de $d_{\text {max }}$ entre os bebês de índices de 1 a 50 não estão homogeneamente dispersos. Além disso, essas figuras apontam que o grupo de bebês com índices até 50 tem valor $d_{\text {max }}$ superior ao do grupo de bebês com índices acima de 50. No banco de dados, verificamos que os recém-nascidos com índices até 50 pertencem ao grupo A termo e os demais recém-nascidos, ao grupo Pré-termo. $\mathrm{Na}$ Seção 6.3.5, vimos que a medida de influência local com esquema de perturbação ponderação de casos também pode ser interpretada com uma perturbação na matriz de correlação de trabalho. Nessa caso, a Figura 8.15e também pode dar um indicativo, por exemplo, de que a matriz de correlação de trabalho deveria ser diferente para cada grupo.

Com o intuito de eliminar essa possível presença de heterogeneidade da precisão, fizemos uma modelagem conjunta da média sob o modelo (8.5) e da precisão sob o modelo:

$$
\log \left(\phi_{i j}\right)=\gamma_{0}+\gamma_{1}\left(\mathrm{x}_{1 i j}\right)
$$




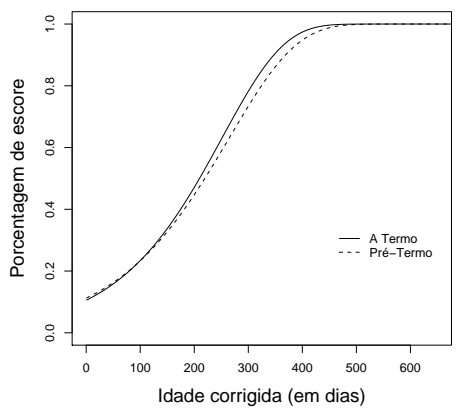

(a)

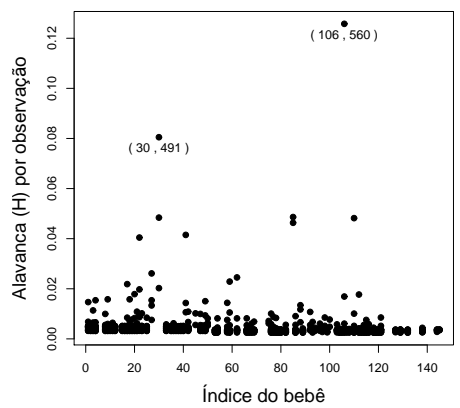

(b)

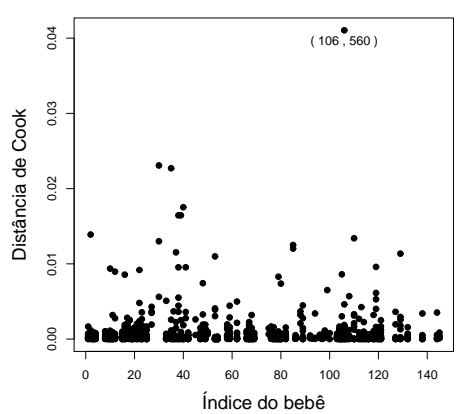

(c)

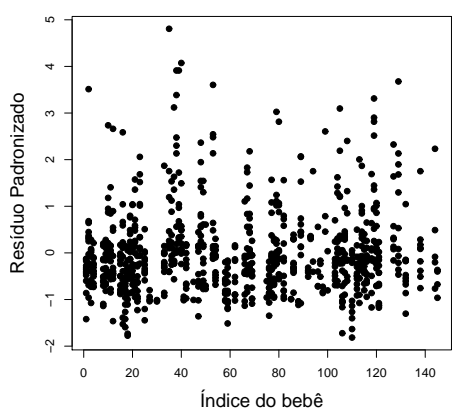

(d)

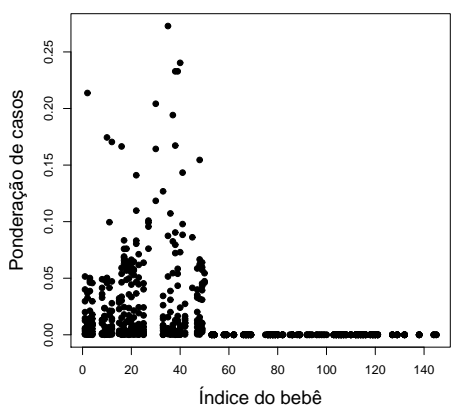

(e)

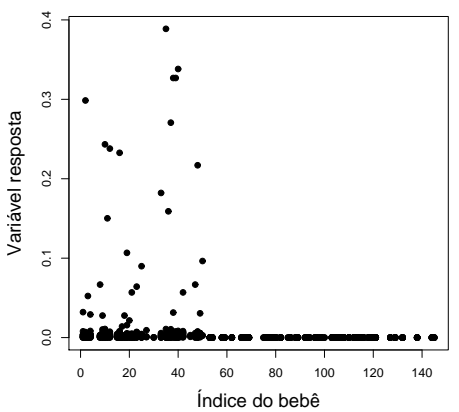

(f)

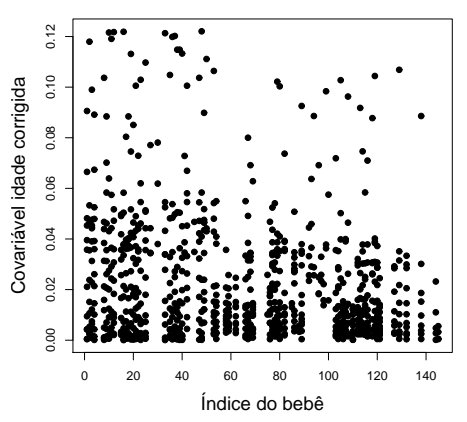

$(\mathrm{g})$

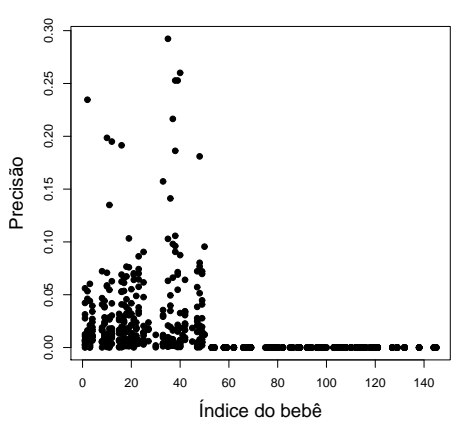

(h)

Figura 8.15: Curvas ajustadas e gráficos de diagnóstico com todas as observações, para modelo de regressão beta sob homogeneidade da dispersão. Estudo de desenvolvimento motor de bebês. 
As estimativas dos parâmetros desse modelo estão na Tabela 8.6 e as curvas ajustadas, na Figura 8.16a. Nessa tabela, observamos significância do modelo sob heterogeneidade da dispersão (nível descritivo, $p<0,001$ ) e as conclusões para os parâmetros $\boldsymbol{\beta}$ são as mesmas feitas para o modelo sob homogeneidade da dispersão.

Tabela 8.6: Estimativas e erros padrões dos parâmetros do modelo de regressão beta sob heterogeneidade da dispersão e com estrutura AR-1. Estudo de desenvolvimento motor de bebês.

\begin{tabular}{ccccrr}
\hline & & & \multicolumn{2}{c}{ Erro Padrão } & \multicolumn{2}{c}{ Nível } \\
\multicolumn{2}{c}{ Parâmetro } & Estimativa & Robusto & Naive & Descritivo \\
\hline$\beta_{0}$ & Intercepto & $-2,5964$ & 0,0539 & 0,0339 & $<0,001$ \\
$\beta_{1}$ & $\mathrm{x}_{1}$ & 0,0291 & 0,0769 & 0,0409 & 0,705 \\
$\beta_{2}$ & $\mathrm{x}_{2}$ & 0,0099 & 0,0002 & 0,0001 & $<0,001$ \\
$\beta_{3}$ & $\mathrm{x}_{1} * \mathrm{x}_{2}$ & $-0,0012$ & 0,0003 & 0,0001 & $<0,001$ \\
$\gamma_{0}$ & Intercepto & 4,3477 & 0,0222 & 0,0070 & $<0,001$ \\
$\gamma_{1}$ & $\mathrm{x}_{1}$ & 0,4238 & 0,0293 & 0,0083 & $<0,001$ \\
$\alpha$ & Correlação & 0,8159 & & & \\
\hline
\end{tabular}

Com a modelagem conjunta da média e da dispersão, tivemos uma redução do resíduo padronizado, Figura 8.16d, quando comparado com o resíduo apresentado na Figura 8.15d. Entretanto, essa modelagem ainda mantém influências distintas dos dois grupos na estimação dos parâmetros de regressão, apesar de agora ser maior a influência do grupo Pré-termo (ver Figura 8.17).

Para avaliar se um modelo mais adequado para esses dados deve ou não ter matriz de correlação de trabalho distinta para cada grupo, ajustamos o modelo para média $\log \left[-\log \left(1-\mu_{i j}\right)\right]=\beta_{0}+\beta_{1}\left(\mathrm{x}_{2 i j}\right)$ considerando distribuição beta, homogeneidade da precisão e estrutura AR-1. Esse modelo foi ajustado separadamente para cada grupo. As estimativas da correlação foram $\hat{\alpha}=0,997$ para o grupo A termo e $\hat{\alpha}=0,253$ para o grupo Pré-termo, possivelmente indicando que um modelo mais adequado a esses dados deveria impor matrizes de correlação de trabalho distintas para cada grupo. Esse tipo de modelagem não é abordada neste trabalho.

\section{Simplex}

Considerando distribuição simplex, função de ligação logito, homogeneidade da dis- 


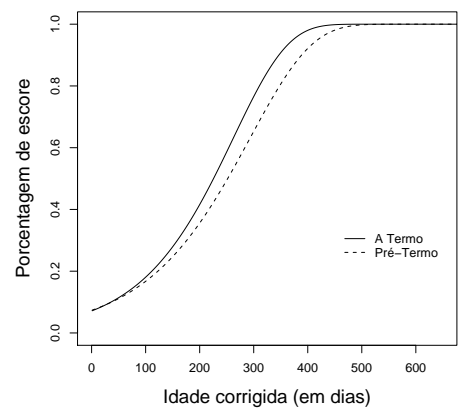

(a)

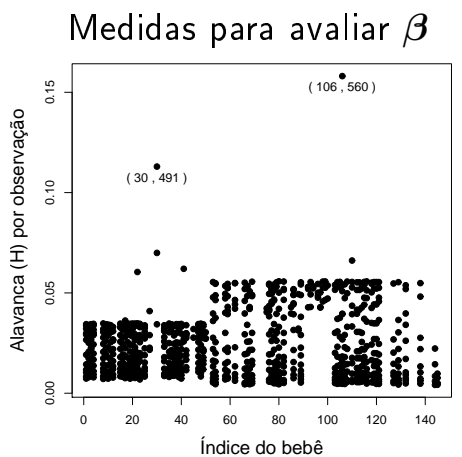

(b)

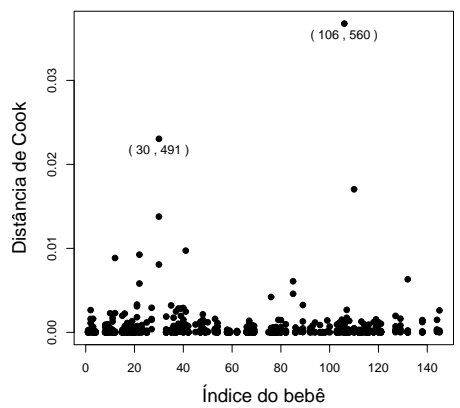

(c)

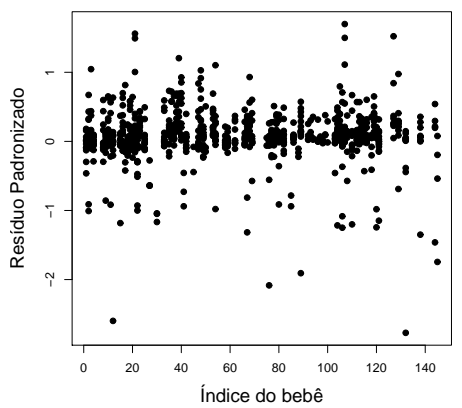

(d)

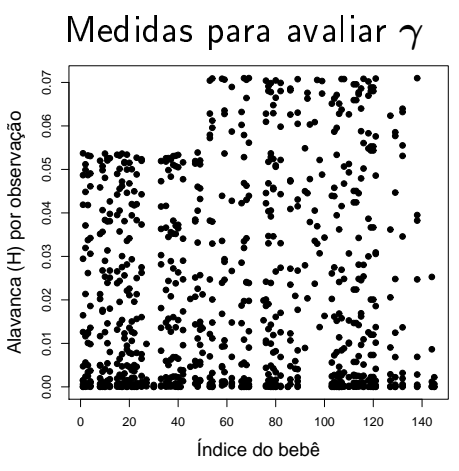

(e)

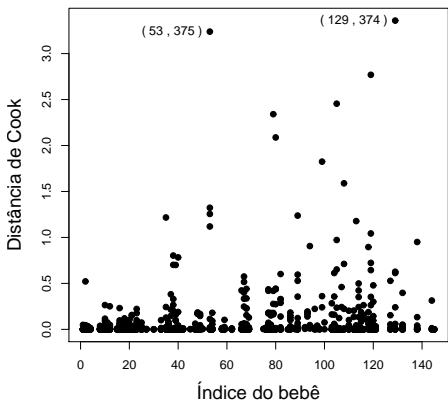

(f)

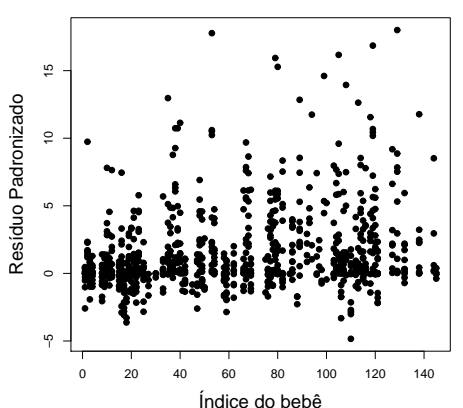

(g)

Figura 8.16: Curvas ajustadas (a); gráficos de diagnóstico do ajuste de $\boldsymbol{\beta}$ (b)-(d) e do ajuste de $\gamma(\mathrm{e})$-(g), para modelos de regressão beta sob heterogeneidade da dispersão e com todas as observações. Estudo de desenvolvimento motor de bebês. 


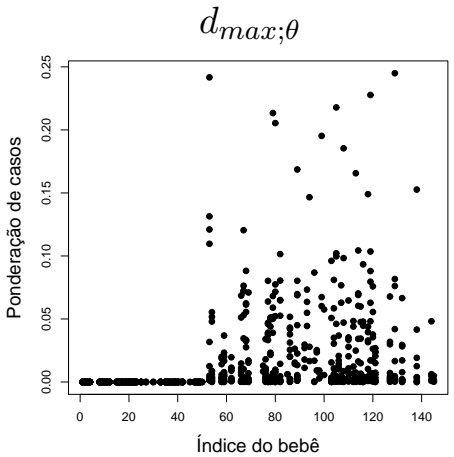

(a)

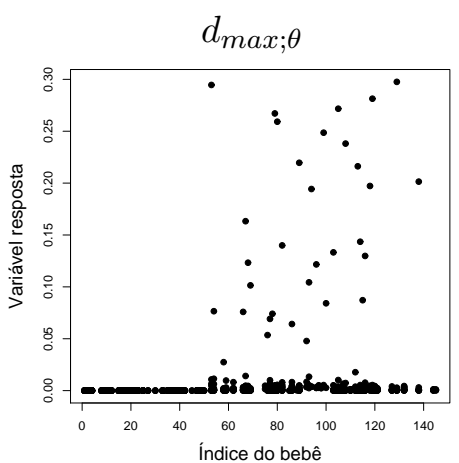

(b)

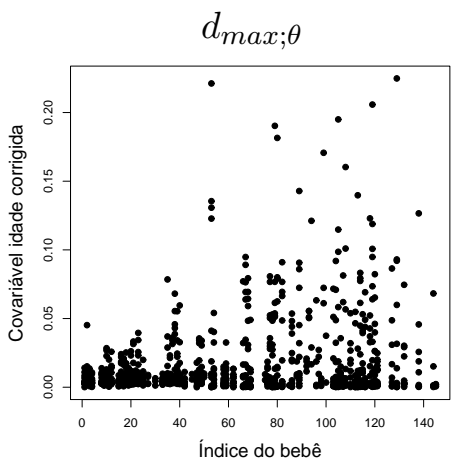

(c)

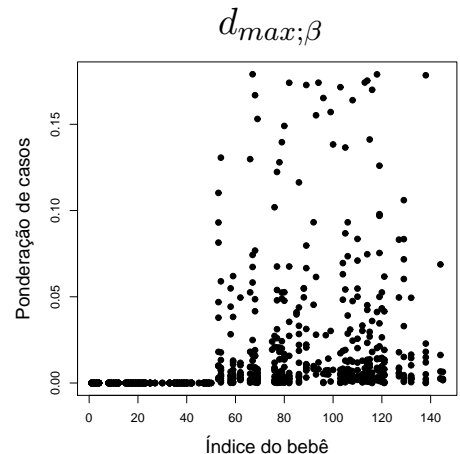

(d)

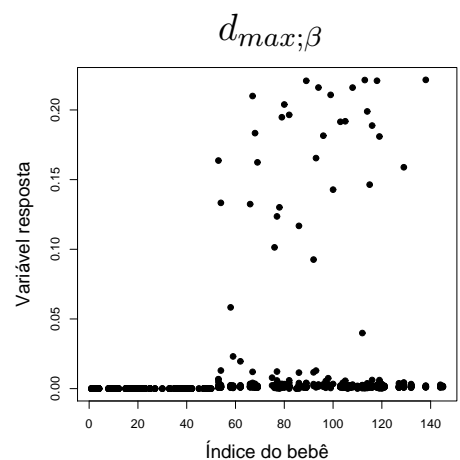

(e)

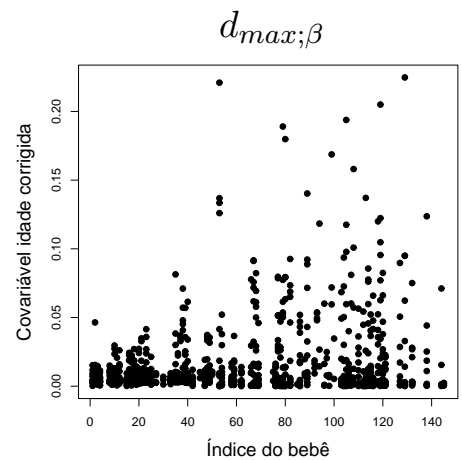

(f)

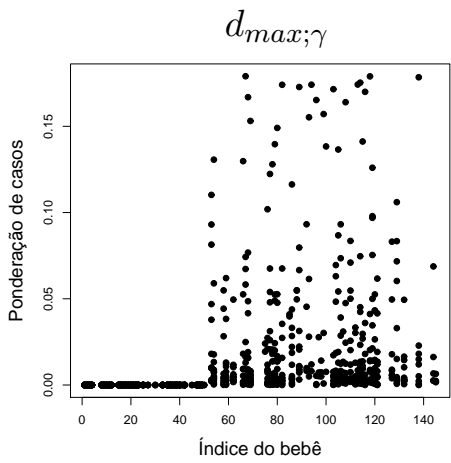

(g)

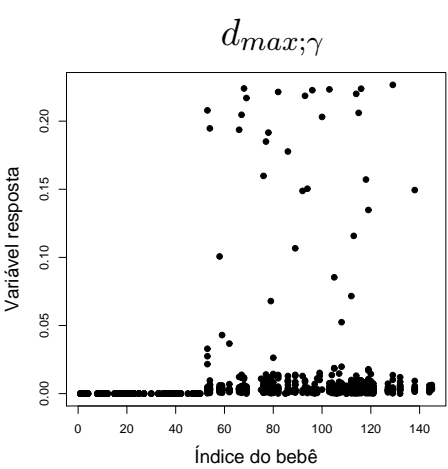

(h)

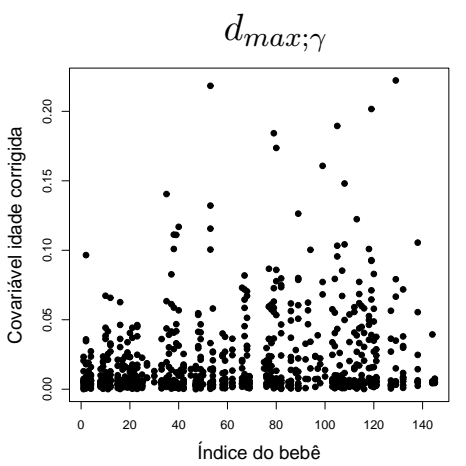

(i)

Figura 8.17: Gráficos de influência local para $\boldsymbol{\theta}$ (a)-(c); para $\boldsymbol{\beta}(\mathrm{d})$-(f) e para $\boldsymbol{\gamma}(\mathrm{g})$-(i), para modelos de regressão beta sob heterogeneidade da dispersão e com todas as observações. Estudo de desenvolvimento motor de bebês. 
persão e estrutura de correlação AR-1, foi ajustado o seguinte modelo:

$$
\log \left(\frac{\mu_{i j}}{1-\mu_{i j}}\right)=\beta_{0}+\beta_{1}\left(\mathrm{x}_{1 i j}\right)+\beta_{2}\left(\mathrm{x}_{2 i j}\right)+\beta_{3}\left(\mathrm{x}_{1 i j} * \mathrm{x}_{2 i j}\right)
$$

com $\mathrm{x}_{1 i j} \mathrm{e}_{2 i j}$ conforme descrito em (8.5).

Pela Tabela 8.7, também observamos significância da interação entre grupo $\left(\mathrm{x}_{1}\right)$ e tempo $\left(\mathrm{x}_{2}\right)$ para descrever o aumento médio do escore atingido com relação à pontuação máxima (nível descritivo, $p=0,001$ ). As curvas ajustadas estão na Figura 8.18a.

Tabela 8.7: Estimativas e erros padrões dos parâmetros do modelo de regressão simplex sob homogeneidade da dispersão e com estrutura AR-1. Estudo de desenvolvimento motor de bebês.

$$
\text { Erro Padrão Nível }
$$

\begin{tabular}{cccccr}
\multicolumn{2}{c}{ Parâmetro } & Estimativa & Robusto & Naive & Descritivo \\
\hline$\beta_{0}$ & Intercepto & $-3,1234$ & 0,0618 & 0,1559 & $<0,001$ \\
$\beta_{1}$ & $\mathrm{x}_{1}$ & 0,4598 & 0,1399 & 0,2020 & 0,001 \\
$\beta_{2}$ & $\mathrm{x}_{2}$ & 0,0169 & 0,0004 & 0,0006 & $<0,001$ \\
$\beta_{3}$ & $\mathrm{x}_{1} * \mathrm{x}_{2}$ & $-0,0030$ & 0,0009 & 0,0008 & $<0,001$ \\
$\phi^{-1}$ & Dispersão & 89,493 & & & \\
$\alpha$ & Correlação & 0,1344 & & & \\
\hline
\end{tabular}

Nas Figuras 8.18b-d apresentamos, respectivamente, a medida de alavanca baseada na matriz de projeção, a distância de Cook e o resíduo padronizado. Esses gráficos destacam, como possíveis pontos alavanca, as observações referentes aos bebês com índice 30 e idade corrigida de 491 dias, $(30,491)$, e com índice 106 e idade corrigida de 560 dias, $(106,560)$. As observações $(30,437),(85,519)$ e $(110,518)$ são destacadas como possíveis pontos influentes e/ou aberrantes.

Nas Figuras 8.18e-h, encontramos os gráficos de influência local, respectivamente, para os esquemas de perturbação: ponderação de casos, da variável resposta, da covariável idade corrigida $\left(\mathrm{x}_{2}\right)$ e da precisão. Essas figuras apontam que o grupo de bebês com índices até 50 (grupo A termo) tem valor $d_{\text {max }}$ inferior ao do grupo de bebês com índices acima de 50 (grupo Pré termo). Além disso, com exceção da figura referente à perturbação da variável resposta, as demais destacam os pontos $(85,519)$ e $(110,518)$ como possíveis pontos influentes, cuja exclusão não altera as conclusões quando comparadas com as do ajuste com todas as observações. 


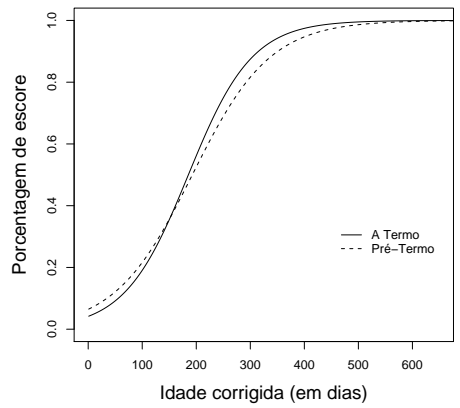

(a)

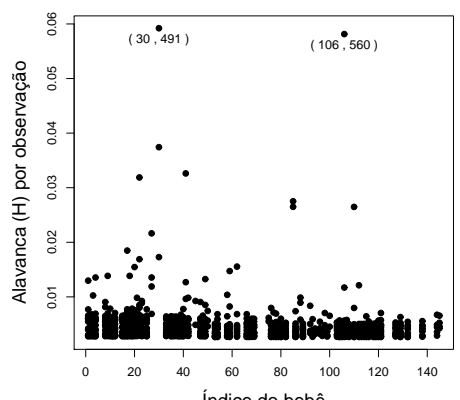

(b)

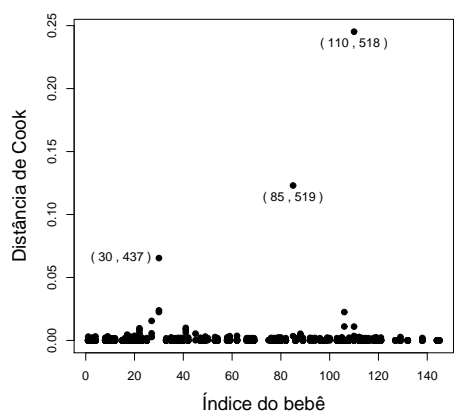

(c)

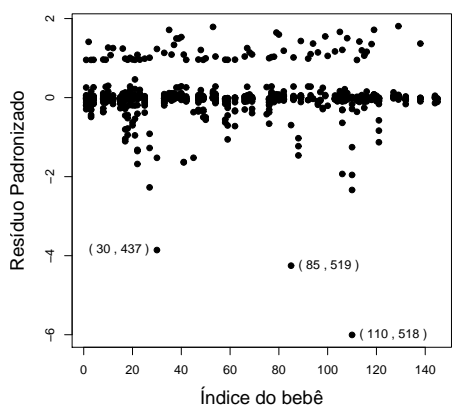

(d)

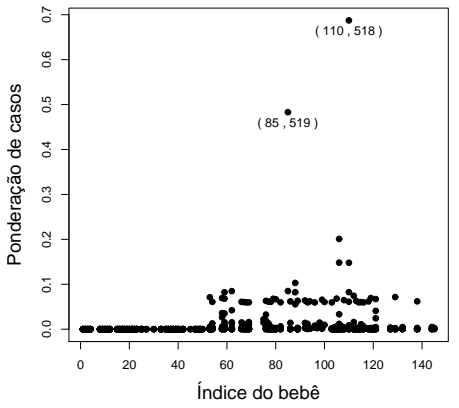

(e)

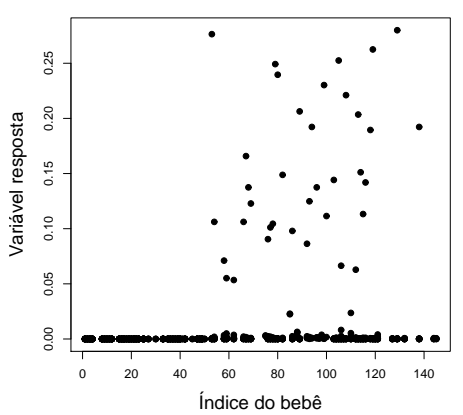

(f)

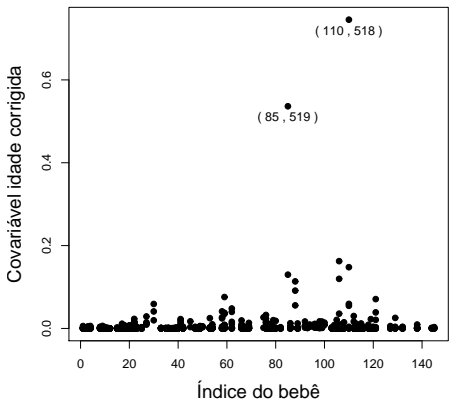

(g)

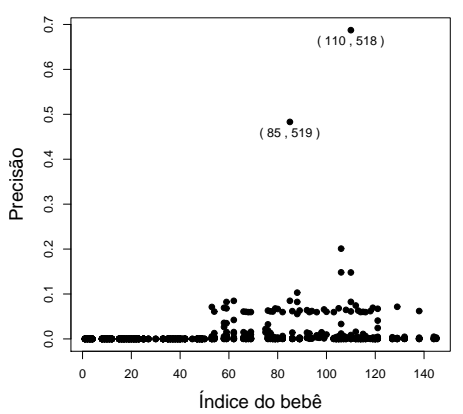

(h)

Figura 8.18: Curvas ajustadas e gráficos de diagnóstico com todas as observações, para modelo de regressão simplex sob homogeneidade da dispersão. Estudo de desenvolvimento motor de bebês. 
A seguir, modelamos a dispersão dos dados para avaliar se isso corrige as diferentes influências de cada grupo nas estimativas dos parâmetros de regressão. A modelagem conjunta da média e da dispersão considerou os modelos (8.6) e $\log \left(\phi_{i j}^{-1}\right)=\gamma_{0}+\gamma_{1}\left(\mathrm{x}_{1 i j}\right)+$ $\gamma_{2}\left(\mathrm{x}_{2 i j}\right)$.

As estimativas dos parâmetros desse modelo estão na Tabela 8.8 e as curvas ajustadas, na Figura 8.19a. Os resultados indicam significância da idade corrigida $\left(\mathrm{x}_{2}\right)$ em ambos modelos (nível descritivo, $p<0,001$ ).

Tabela 8.8: Estimativas e erros padrões dos parâmetros do modelo de regressão simplex sob heterogeneidade da dispersão e com estrutura AR-1. Estudo de desenvolvimento motor de bebês.

\begin{tabular}{cccccr}
\hline & & & \multicolumn{2}{c}{ Erro Padrão } & \multicolumn{1}{c}{ Nível } \\
\cline { 3 - 5 } Parâmetro & Estimativa & Robusto & Naive & Descritivo \\
\hline$\beta_{0}$ & Intercepto & $-2,7341$ & 0,0688 & 0,0413 & $<0,001$ \\
$\beta_{1}$ & $\mathrm{x}_{1}$ & $-0,0400$ & 0,0880 & 0,0472 & 0,654 \\
$\beta_{2}$ & $\mathrm{x}_{2}$ & 0,0146 & 0,0003 & 0,0004 & $<0,001$ \\
$\beta_{3}$ & $\mathrm{x}_{1} * \mathrm{x}_{2}$ & $-0,0006$ & 0,0004 & 0,0004 & 0,165 \\
$\gamma_{0}$ & Intercepto & $-1,2776$ & 0,3104 & 0,1161 & $<0,001$ \\
$\gamma_{1}$ & $\mathrm{x}_{1}$ & $-0,1762$ & 0,1706 & 0,0991 & 0,302 \\
$\gamma_{2}$ & $\mathrm{x}_{2}$ & 0,0163 & 0,0011 & 0,0004 & $<0,001$ \\
$\alpha$ & Correlação & 0,2951 & & & \\
\hline
\end{tabular}

Com a modelagem conjunta da média e da dispersão, tivemos um aumento do resíduo padronizado, Figura 8.19d, quando comparado com o resíduo apresentado na Figura 8.18d e continuou mantendo influências distintas dos dois grupos na estimação dos parâmetros de regressão da média (ver Figura 8.20d). Essas interpretações continuam prevalecendo mesmo com a exclusão dos possíveis pontos influentes destacados na Figura 8.20.

Da mesma maneira que ocorreu com a distribuição beta, o ajuste utilizando a distribuição simplex também dá um indicativo de que a matriz de correlação de trabalho deveria ser diferente para cada um dos grupos. Considerando distribuição simplex, homogeneidade da precisão e estrutura AR-1, o modelo para média $\log \left(\mu_{i j} /\left(1-\mu_{i j}\right)\right)=\beta_{0}+\beta_{1}\left(\mathrm{x}_{2 i j}\right)$ foi ajustado separadamente para cada grupo. As estimativas da correlação foram $\hat{\alpha}=-0,094$ para o grupo A termo e $\hat{\alpha}=0,367$ para o grupo Pré termo. Lembramos que, para a distribuição simplex, a matriz de correlação de trabalho não reflete a correlação das variáveis respostas $\mathbf{y}$, mas do vetor $\mathbf{u}$ conforme definido na equação (3.1). 


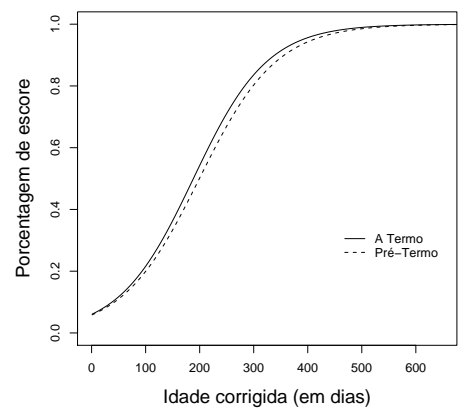

(a)

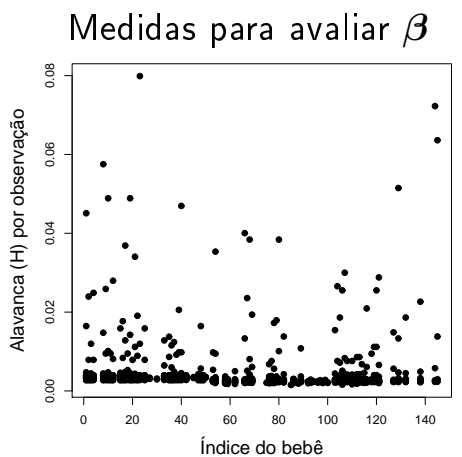

(b)

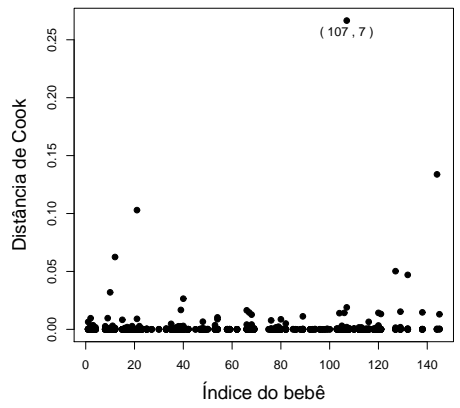

(c)

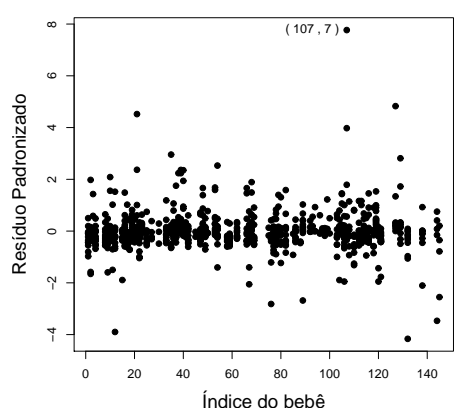

(d)

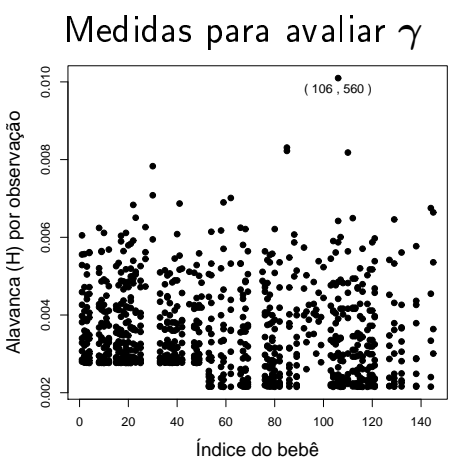

(e)

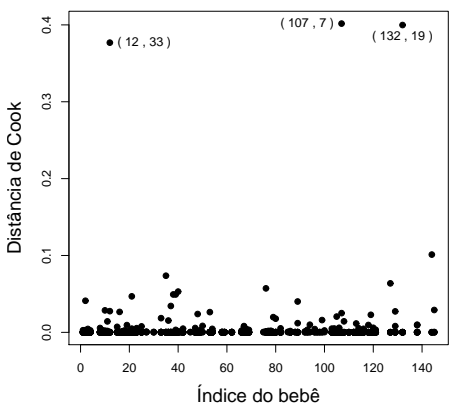

(f)

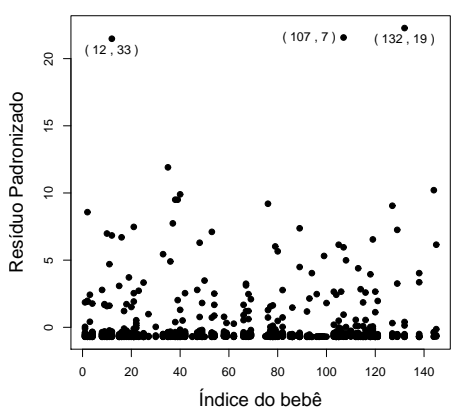

(g)

Figura 8.19: Curvas ajustadas (a); gráficos de diagnóstico do ajuste de $\boldsymbol{\beta}$ (b)-(d) e do ajuste de $\gamma(\mathrm{e})-(\mathrm{g})$, para modelos de regressão simplex sob heterogeneidade da dispersão e com todas as observações. Estudo de desenvolvimento motor de bebês. 


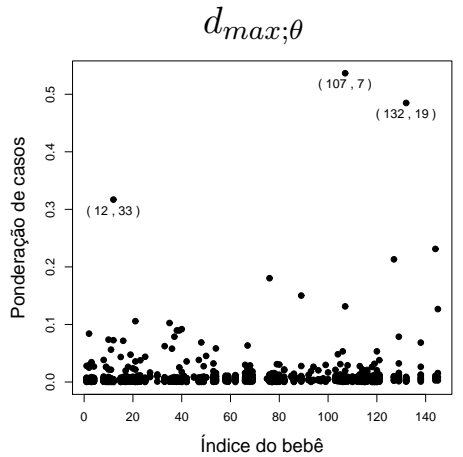

(a)

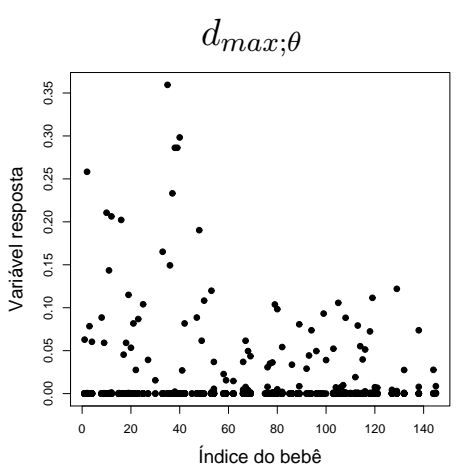

(b)

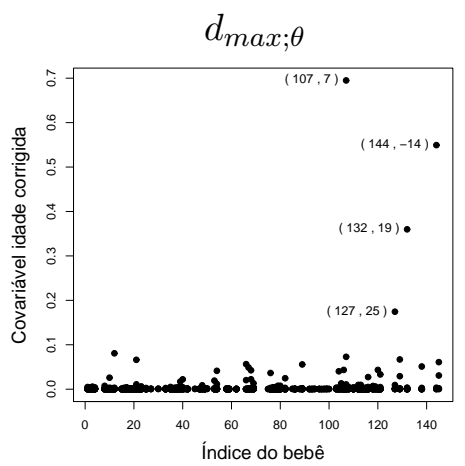

(c)

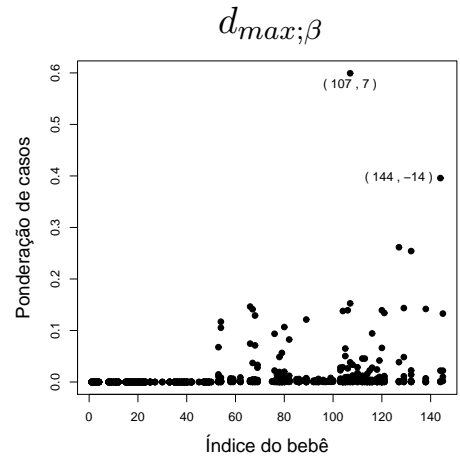

(d)

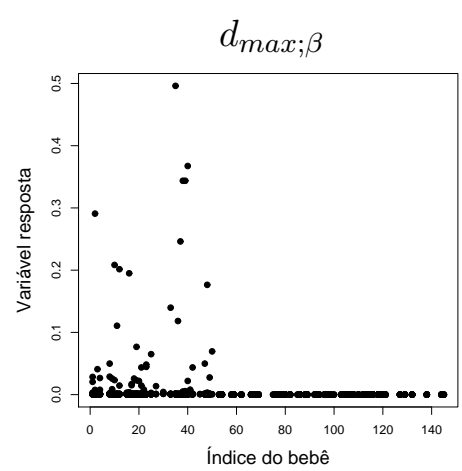

(e)

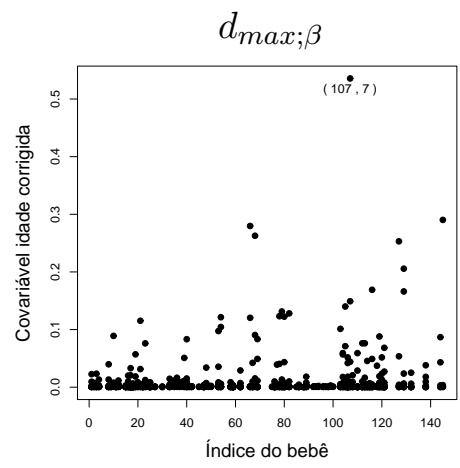

(f)

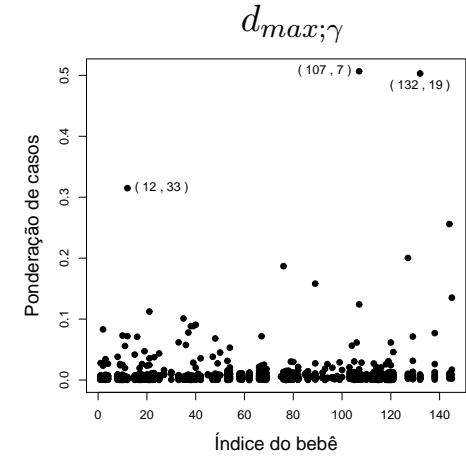

(g)

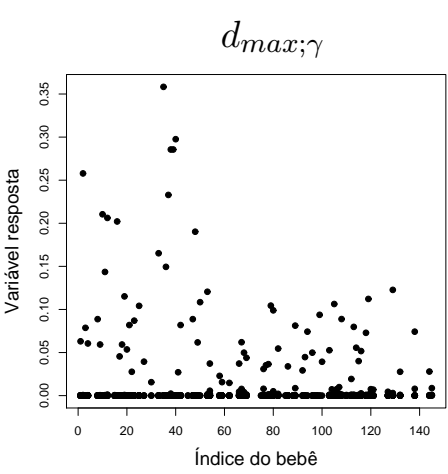

(h)

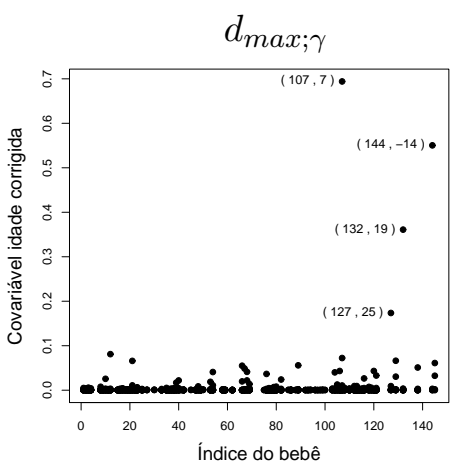

(i)

Figura 8.20: Gráficos de influência local para $\boldsymbol{\theta}$ (a)-(c); para $\boldsymbol{\beta}(\mathrm{d})-(\mathrm{f})$ e para $\boldsymbol{\gamma}(\mathrm{g})-(\mathrm{i})$, para modelos de regressão simplex sob heterogeneidade da dispersão e com todas as observações. Estudo dos bebês. 


\section{Capítulo 9}

\section{Conclusões e Estudos futuros}

Na primeira parte deste trabalho, propomos equações de estimação para análise de dados com medidas repetidas considerando distribuições beta, simplex e aquelas pertencentes à família exponencial. Todas essas equações foram desenvolvidas segundo a proposta de Liang e Zeger (1986).

Para estudos futuros, com relação à construção de novas equações de estimação, citamos as seguintes linhas de pesquisa:

- Ospina e Ferrari (2008) propõem distribuições mistas contínua-discreta para modelar dados pertencentes ao intervalo fechado [0,1], com probabilidade positiva em ao menos um dos extremos. Os autores discutem algumas propriedades dessas distribuições e propõem estimação pelos métodos de máxima verossimilhança e dos momentos. Nesse sentido, indicamos a extensão das equações de estimação para dados com medidas repetidas e distribuições que consideram respostas pertencentes aos intervalos $[0,1),(0,1]$ ou $[0,1]$.

- Para construir as equações de estimação propostas na primeira parte deste trabalho, definimos uma mesma matriz de correlação de trabalho, $\mathbf{R}(\boldsymbol{\alpha})$, para todas as unidades experimentais $i$, com $i=1, \ldots, n$. Entretanto, a conclusão obtida com a Aplicação II do Capítulo 8 foi que assumir matrizes de correlação de trabalho diferentes para cada um dos grupos (A termo e Pré-termo) seria mais indicada para modelagem dos dados dos bebês. Nesse caso, para ajustar modelos que não consideram, necessariamente, uma única matriz de correlação para todas as unidades experimentais, indicamos duas extensões:

- construir equações de estimação considerando matrizes de correlação $\mathbf{R}_{g}(\boldsymbol{\alpha})$, sendo $g$ o índice de grupo. Essas matrizes podem ser estimadas, via métodos 
dos momentos, usando esse índice $g$ nos estimadores para $\boldsymbol{\alpha}$ propostos na Seção 2.1.1.

- construir equações de estimação considerando uma modelagem conjunta da média e do parâmetro de correlação ou ainda uma modelagem conjunta da média e dos parâmetros de dispersão e de correlação. Preisser e Perin (2007) propõem um componente sistemático que modela o parâmetro de correlação.

Na Parte II, desenvolvemos técnicas de diagnóstico tanto para modelagem da média sob suposição de homogeneidade da dispersão como para modelagem conjunta da média e do parâmetro de dispersão. Entre as medidas desenvolvidas, temos a matriz de projeção para detectar pontos alavanca, a distância de Cook e a influência local para detectar pontos influentes na estimação dos parâmetros de regressão e a medida $Q I C$ utilizada para selecionar uma matriz de correlação de trabalho.

Em estudos futuros, com relação ao desenvolvimento de novas técnicas de diagnóstico, citamos as seguintes abordagens:

- Para avaliarmos o ajuste de um modelo, Atkinson (1985) propõe o gráfico de probabilidade meio-normal com envelope simulado. Esse gráfico pode ser utilizado mesmo que os resíduos não tenham distribuição de probabilidade normal (Neter et al., 1996), bastando apenas gerar dados com a mesma distribuição atribuída ao modelo ajustado. Para fazer uso desse gráfico nos modelos propostos neste trabalho, é necessário desenvolver algoritmos para simular variáveis correlacionadas com distribuições marginais beta, simplex e outras pertencentes à família exponencial que não foram citadas neste trabalho. Uma sugestão seria trabalhar com a teoria de cópulas (Joe, 1997) que é um método para construir distribuições multivariadas a partir das distribuições marginais das observações e da estrutura de dependência entre elas.

- A medida $Q I C$ apresentada na Seção 5.2 foi proposta por Pan (2001) apenas no enfoque em que assumimos homogeneidade do parâmetro de dispersão. Uma nova proposta é a construção de uma medida $Q I C$ que considera a modelagem do parâmetro de dispersão.

- Para estender o conceito de alavanca, originalmente definido no modelo de regressão normal linear, Wei et al. (1998) buscaram captar o sentido essencial desse termo em Estatística. Baseados no ponto de vista de que uma medida de alavancagem deve refletir diretamente a influência da resposta observada no próprio valor ajustado, os autores propõem a matriz de alavanca generalizada, generalized leverage, para estimadores dos parâmetros de regressão. Além disso, discutem a conexão entre essa 
medida e a de influência local (Cook, 1986) com esquema de perturbação da variável resposta. Nesse caso, indicamos a extensão da matriz de alavanca generalizada para equações de estimação considerando dados com medidas repetidas e estabelecer uma relação entre essa medida e a de influência local apresentada na Seção 6.2. 


\section{Apêndice A}

\section{Detalhes para obtenção das equações}

\section{de estimação}

Neste apêndice, apresentamos cálculos detalhados desenvolvidos para obtenção das equações de estimação descritas na primeira parte deste trabalho.

\section{A.1 Modelos de regressão beta}

\section{A.1.1 EEGs sob homogeneidade da dispersão}

As contas detalhadas para obtenção da equação de estimação ótima descrita em (2.3) são dadas por

$$
\begin{aligned}
\mathrm{E}\left(\frac{\partial \mathbf{b}_{i}}{\partial \boldsymbol{\beta}^{\top}}\right)^{\top} & =\mathrm{E}\left(\frac{\partial}{\partial \boldsymbol{\beta}^{\top}}\left(\mathbf{y}_{i}^{*}-\boldsymbol{\mu}_{i}^{*}\right)\right)^{\top}=-\left(\frac{\partial \boldsymbol{\mu}_{i}^{*}}{\partial \boldsymbol{\mu}_{i}} \frac{\partial \boldsymbol{\mu}_{i}}{\partial \boldsymbol{\eta}_{i}} \frac{\partial \boldsymbol{\eta}_{i}}{\partial \boldsymbol{\beta}^{\top}}\right)^{\top}= \\
& =-\mathbf{X}_{i}^{\top} \mathbf{G}_{i} \operatorname{diag}\left(\partial \mu_{i 1}^{*} / \partial \mu_{i 1}, \ldots, \partial \mu_{i t}^{*} / \partial \mu_{i t}\right)= \\
& =-\phi \mathbf{X}_{i}^{\top} \mathbf{G}_{i} \mathbf{A}_{i}=-\mathbf{X}_{i}^{\top} \boldsymbol{\Lambda}_{i},
\end{aligned}
$$

sendo

$$
\begin{aligned}
& \mathbf{X}_{i}=\left(\mathbf{x}_{i 1}, \ldots, \mathbf{x}_{i t}\right)^{\top}, \quad \text { com dimensão }(t \times p), \\
& \mathbf{G}_{i}=\operatorname{diag}\left(\partial g^{-1}\left(\eta_{i 1}\right) / \partial \eta_{i 1}, \ldots, \partial g^{-1}\left(\eta_{i t}\right) / \partial \eta_{i t}\right), \quad \text { com dimensão }(t \times t) \mathrm{e} \\
& \mathbf{A}_{i}=\operatorname{diag}\left(\mathrm{a}_{i 1}, \ldots, \mathbf{a}_{i t}\right), \quad \text { com dimensão }(t \times t),
\end{aligned}
$$

$\operatorname{com} \mathrm{a}_{i j}=\psi^{\prime}\left(\mu_{i j} \phi\right)+\psi^{\prime}\left(\left(1-\mu_{i j}\right) \phi\right), i=1, \ldots, n$ e $j=1, \ldots, t$. 
A matriz de covariâncias de $\mathbf{b}_{i}=\mathbf{y}_{i}^{*}-\boldsymbol{\mu}_{i}^{*}$ pode ser escrita na forma

$$
\operatorname{Cov}\left(\mathbf{b}_{i}\right)=\operatorname{Var}\left(\mathbf{b}_{i}\right)^{1 / 2} \mathbf{R}\left(\mathbf{b}_{i}\right) \operatorname{Var}\left(\mathbf{b}_{i}\right)^{1 / 2}
$$

em que $\mathbf{R}\left(\mathbf{b}_{i}\right)$ representa a matriz de correlação de $\mathbf{b}_{i}$ e $\operatorname{Var}\left(\mathbf{b}_{i}\right)$ é dada por

$$
\operatorname{Var}\left(\mathbf{b}_{i}\right)=\operatorname{diag}\left(\operatorname{Var}\left(\mathrm{b}_{i 1}\right), \ldots, \operatorname{Var}\left(\mathrm{b}_{i t}\right)\right)=\operatorname{diag}\left(\mathrm{E}\left(\mathrm{b}_{i 1}^{2}\right), \ldots, \mathrm{E}\left(\mathrm{b}_{i t}^{2}\right)\right)=\mathbf{A}_{i}
$$

já que

$$
\begin{aligned}
\mathrm{E}\left(\mathrm{b}_{i j}^{2}\right) & =\mathrm{E}\left(\left(\mathrm{y}_{i j}^{*}-\mu_{i j}^{*}\right)^{2}\right)=\phi^{-2} \mathrm{E}\left(\phi^{2}\left(\mathrm{y}_{i j}^{*}-\mu_{i j}^{*}\right)^{2}\right)= \\
& =\phi^{-2} \mathrm{E}\left(\left[\frac{\partial \ell\left(\mu_{i j}, \phi\right)}{\partial \mu_{i j}}\right]^{2}\right)=-\phi^{-2} \mathrm{E}\left(\frac{\partial^{2} \ell\left(\mu_{i j}, \phi\right)}{\partial \mu_{i j}^{2}}\right)= \\
& =\psi^{\prime}\left(\mu_{i j} \phi\right)+\psi^{\prime}\left(\left(1-\mu_{i j}\right) \phi\right)=\mathrm{a}_{i j} .
\end{aligned}
$$

$\operatorname{Logo}, \operatorname{Cov}\left(\mathbf{b}_{i}\right)=\mathbf{A}_{i}^{1 / 2} \mathbf{R}\left(\mathbf{b}_{i}\right) \mathbf{A}_{i}^{1 / 2}=\mathbf{A}_{i}^{1 / 2} \mathbf{R}\left(\mathbf{y}_{i}^{*}\right) \mathbf{A}_{i}^{1 / 2}$.

\section{A.1.2 EEGs sob heterogeneidade da dispersão}

A seguir, apresentamos algumas contas detalhadas para obtenção da equação de estimação ótima descrita em (2.11).

$$
\begin{aligned}
\boldsymbol{\Psi}_{2}^{o}(\boldsymbol{\theta}) & =\sum_{i=1}^{n} \mathrm{E}\left(\frac{\partial \mathbf{b}_{i}}{\partial \boldsymbol{\theta}^{\top}}\right)^{\top} \operatorname{Cov}\left(\mathbf{b}_{i}\right)^{-1} \mathbf{b}_{i}= \\
& =\sum_{i=1}^{n}\left(\mathrm{E}\left(\frac{\partial \mathbf{b}_{i}}{\partial \boldsymbol{\beta}^{\top}}\right) \quad \mathrm{E}\left(\frac{\partial \mathbf{b}_{i}}{\partial \boldsymbol{\gamma}^{\top}}\right)\right)^{\top} \operatorname{Cov}\left(\mathbf{b}_{i}\right)^{-1} \mathbf{b}_{i},
\end{aligned}
$$

sendo

$$
\begin{aligned}
\mathrm{E}\left(\frac{\partial \mathbf{b}_{i}}{\partial \boldsymbol{\beta}^{\top}}\right)^{\top} & =\mathrm{E}\left(\frac{\partial}{\partial \boldsymbol{\beta}^{\top}}\left(\mathbf{y}_{i}^{*}-\boldsymbol{\mu}_{i}^{*}\right)\right)^{\top}=-\left(\frac{\partial \boldsymbol{\mu}_{i}^{*}}{\partial \boldsymbol{\mu}_{i}} \frac{\partial \boldsymbol{\mu}_{i}}{\partial \boldsymbol{\eta}_{i}} \frac{\partial \boldsymbol{\eta}_{i}}{\partial \boldsymbol{\beta}^{\top}}\right)^{\top}= \\
& =-\mathbf{X}_{i}^{\top} \mathbf{G}_{i} \operatorname{diag}\left(\partial \mu_{i 1}^{*} / \partial \mu_{i 1}, \ldots, \partial \mu_{i t}^{*} / \partial \mu_{i t}\right)= \\
& =-\mathbf{X}_{i}^{\top} \mathbf{G}_{i} \mathbf{\Phi}_{i} \mathbf{A}_{i},
\end{aligned}
$$

$\mathrm{e}$

$$
\begin{aligned}
\mathrm{E}\left(\frac{\partial \mathbf{b}_{i}}{\partial \boldsymbol{\gamma}^{\top}}\right)^{\top} & =\mathrm{E}\left(\frac{\partial}{\partial \boldsymbol{\gamma}^{\top}}\left(\mathbf{y}_{i}^{*}-\boldsymbol{\mu}_{i}^{*}\right)\right)^{\top}=-\left(\frac{\partial \boldsymbol{\mu}_{i}^{*}}{\partial \boldsymbol{\phi}_{i}} \frac{\partial \boldsymbol{\phi}_{i}}{\partial \boldsymbol{\delta}_{i}} \frac{\partial \boldsymbol{\delta}_{i}}{\partial \boldsymbol{\gamma}^{\top}}\right)^{\top} \\
& =-\mathbf{Q}_{i}^{\top} \mathbf{F}_{i} \operatorname{diag}\left(\partial \mu_{i 1}^{*} / \partial \phi_{i 1}, \ldots, \partial \mu_{i t}^{*} / \partial \phi_{i t}\right)= \\
& =-\mathbf{Q}_{i}^{\top} \mathbf{F}_{i} \mathbf{C}_{i}
\end{aligned}
$$


em que

$\boldsymbol{\Phi}_{i}=\operatorname{diag}\left(\phi_{i 1}, \ldots, \phi_{i t}\right), \quad$ com dimensão $(t \times t)$,

$\mathbf{A}_{i}=\operatorname{diag}\left(\mathrm{a}_{i 1}, \ldots, \mathrm{a}_{i t}\right), \quad$ com dimensão $(t \times t)$,

$\mathbf{Q}_{i}=\left(\mathbf{q}_{i 1}, \ldots, \mathbf{q}_{i t}\right)^{\top}, \quad$ com dimensão $(t \times q)$,

$\mathbf{F}_{i}=\operatorname{diag}\left(\partial f^{-1}\left(\delta_{i 1}\right) / \partial \delta_{i 1}, \ldots, \partial f^{-1}\left(\delta_{i t}\right) / \partial \delta_{i t}\right), \quad$ com dimensão $(t \times t) \mathrm{e}$

$\mathbf{C}_{i}=\operatorname{diag}\left(\mathrm{c}_{i 1}, \ldots, \mathrm{c}_{i t}\right), \quad$ com dimensão $(t \times t)$,

$\operatorname{com} \mathrm{a}_{i j}=\psi^{\prime}\left(\mu_{i j} \phi_{i j}\right)+\psi^{\prime}\left(\left(1-\mu_{i j}\right) \phi_{i j}\right)$ e $\mathrm{c}_{i j}=\mu_{i j} \psi^{\prime}\left(\mu_{i j} \phi_{i j}\right)-\left(1-\mu_{i j}\right) \psi^{\prime}\left(\left(1-\mu_{i j}\right) \phi_{i j}\right)$, $i=1, \ldots, n$ e $j=1, \ldots, t$.

A matriz de sensibilidade e a matriz de variabilidade para a equação de estimação generalizada descrita em (2.12) são, respectivamente, dadas por

$$
\begin{aligned}
& \sum_{i=1}^{n} \mathbf{S}_{i}=\mathrm{E}\left(\frac{\partial}{\partial \boldsymbol{\theta}^{\top}} \boldsymbol{\Psi}_{2}(\boldsymbol{\theta})\right)=\mathrm{E}\left(\begin{array}{cc}
\frac{\partial}{\partial \beta^{\top}} \boldsymbol{\Psi}_{2}(\boldsymbol{\beta}) & \frac{\partial}{\partial \gamma^{\top}} \boldsymbol{\Psi}_{2}(\boldsymbol{\beta}) \\
\frac{\partial}{\partial \beta^{\top}} \boldsymbol{\Psi}_{2}(\gamma) & \frac{\partial}{\partial \gamma^{\top}} \boldsymbol{\Psi}_{2}(\boldsymbol{\gamma})
\end{array}\right)= \\
& =-\sum_{i=1}^{n}\left(\begin{array}{cc}
\mathbf{X}_{i}^{\top} \mathbf{G}_{i} \boldsymbol{\Phi}_{i} \mathbf{A}_{i} \boldsymbol{\Omega}_{i}^{-1} \mathbf{A}_{i} \boldsymbol{\Phi}_{i} \mathbf{G}_{i} \mathbf{X}_{i} & \mathbf{X}_{i}^{\top} \mathbf{G}_{i} \boldsymbol{\Phi}_{i} \mathbf{A}_{i} \boldsymbol{\Omega}_{i}^{-1} \mathbf{C}_{i} \mathbf{F}_{i} \mathbf{Q}_{i} \\
\mathbf{Q}_{i}^{\top} \mathbf{F}_{i} \mathbf{C}_{i} \boldsymbol{\Omega}_{i}^{-1} \mathbf{A}_{i} \boldsymbol{\Phi}_{i} \mathbf{G}_{i} \mathbf{X}_{i} & \mathbf{Q}_{i}^{\top} \mathbf{F}_{i} \mathbf{C}_{i} \boldsymbol{\Omega}_{i}^{-1} \mathbf{C}_{i} \mathbf{F}_{i} \mathbf{Q}_{i}
\end{array}\right)= \\
& =-\sum_{i=1}^{n} \mathbf{M}_{i}^{\top} \boldsymbol{\Lambda}_{i} \boldsymbol{\Omega}^{-1} \boldsymbol{\Lambda}_{i}^{\top} \mathbf{M}_{i}
\end{aligned}
$$

$\mathrm{e}$

$$
\begin{aligned}
& \sum_{i=1}^{n} \mathbf{V}_{i}=\mathrm{E}\left(\boldsymbol{\Psi}_{2}(\boldsymbol{\theta}) \boldsymbol{\Psi}_{2}^{\top}(\boldsymbol{\theta})\right)=\mathrm{E}\left(\begin{array}{ll}
\boldsymbol{\Psi}_{2}(\boldsymbol{\beta}) \boldsymbol{\Psi}_{2}^{\top}(\boldsymbol{\beta}) & \boldsymbol{\Psi}_{2}(\boldsymbol{\beta}) \boldsymbol{\Psi}_{2}^{\top}(\boldsymbol{\gamma}) \\
\boldsymbol{\Psi}_{2}(\boldsymbol{\gamma}) \boldsymbol{\Psi}_{2}^{\top}(\boldsymbol{\beta}) & \boldsymbol{\Psi}_{2}(\boldsymbol{\gamma}) \boldsymbol{\Psi}_{2}^{\top}(\boldsymbol{\gamma})
\end{array}\right)= \\
& =\sum_{i=1}^{n}\left(\begin{array}{c}
\mathbf{X}_{i}^{\top} \mathbf{G}_{i} \boldsymbol{\Phi}_{i} \mathbf{A}_{i} \\
\mathbf{Q}_{i}^{\top} \mathbf{F}_{i} \mathbf{C}_{i}
\end{array}\right) \boldsymbol{\Omega}_{i}^{-1} \operatorname{Cov}\left(\mathbf{b}_{i}\right) \boldsymbol{\Omega}_{i}^{-1}\left(\begin{array}{ll}
\mathbf{A}_{i} \boldsymbol{\Phi}_{i} \mathbf{G}_{i} \mathbf{X}_{i} & \mathbf{C}_{i} \mathbf{F}_{i} \mathbf{Q}_{i}
\end{array}\right)= \\
& =\sum_{i=1}^{n} \mathbf{M}_{i}^{\top} \boldsymbol{\Lambda}_{i} \boldsymbol{\Omega}_{i}^{-1} \operatorname{Cov}\left(\mathbf{b}_{i}\right) \boldsymbol{\Omega}_{i}^{-1} \boldsymbol{\Lambda}_{i}^{\top} \mathbf{M}_{i}
\end{aligned}
$$

sendo $\operatorname{Cov}\left(\mathbf{b}_{i}\right)=\mathrm{E}\left(\mathbf{b}_{i} \mathbf{b}_{i}^{\top}\right)$, já que $\mathrm{E}\left(\mathbf{b}_{i}\right)=\mathbf{0}$. Ressaltamos que as matrizes de sensibilidade e variabilidade têm dimensão $(p+q) \times(p+q)$. 


\section{A.2 Modelos de regressão simplex}

\section{A.2.1 EEGs sob homogeneidade da dispersão}

As contas detalhadas para obtenção da equação de estimação ótima descrita em (3.2) são dadas por

$$
\begin{aligned}
\mathrm{E}\left(\frac{\partial \mathbf{b}_{1 i}}{\partial \boldsymbol{\beta}^{\top}}\right)^{\top} & =\mathrm{E}\left(\frac{\partial \mathbf{u}_{i}}{\partial \boldsymbol{\beta}^{\top}}\right)^{\top}=\mathrm{E}\left(\frac{\partial \mathbf{u}_{i}}{\partial \boldsymbol{\mu}_{i}} \frac{\partial \boldsymbol{\mu}_{i}}{\partial \boldsymbol{\eta}_{i}} \frac{\partial \boldsymbol{\eta}_{i}}{\partial \boldsymbol{\beta}_{i}^{\top}}\right)^{\top}= \\
& =\mathbf{X}_{i}^{\top} \mathbf{G}_{i} \operatorname{diag}\left(\mathrm{E}\left(\partial \mathrm{u}_{i 1} / \partial \mu_{i 1}\right), \ldots, \mathrm{E}\left(\partial \mathrm{u}_{i j} / \partial \mu_{i j}\right)\right)=-\phi \mathbf{X}_{i}^{\top} \mathbf{G}_{i} \mathbf{A}_{i}=-\mathbf{X}_{i} \boldsymbol{\Lambda}_{i}
\end{aligned}
$$

e

$$
\operatorname{Cov}\left(\mathbf{b}_{1 i}\right)=\operatorname{Cov}\left(\mathbf{u}_{i}\right)=\operatorname{Var}\left(\mathbf{u}_{i}\right)^{1 / 2} \mathbf{R}\left(\mathbf{u}_{i}\right) \operatorname{Var}\left(\mathbf{u}_{i}\right)^{1 / 2}=\mathbf{A}_{i}^{1 / 2} \mathbf{R}\left(\mathbf{u}_{i}\right) \mathbf{A}_{i}^{1 / 2},
$$

sendo

$$
\begin{aligned}
& \mathbf{X}_{i}=\left(\mathbf{x}_{i 1}, \ldots, \mathbf{x}_{i t}\right)^{\top}, \quad \text { com dimensão }(t \times p), \\
& \mathbf{G}_{i}=\operatorname{diag}\left(\partial g^{-1}\left(\eta_{i 1}\right) / \partial \eta_{i 1}, \ldots, \partial g^{-1}\left(\eta_{i t}\right) / \partial \eta_{i t}\right), \quad \text { com dimensão }(t \times t) \mathrm{e} \\
& \mathbf{A}_{i}=\operatorname{diag}\left(\mathrm{a}_{i 1}, \ldots, \mathbf{a}_{i t}\right), \quad \text { com dimensão }(t \times t),
\end{aligned}
$$

com $\mathrm{a}_{i j}=\phi^{-1}\left[3 \phi^{-1} /\left(\mu_{i j}\left(1-\mu_{i j}\right)\right)+1 /\left(\mu_{i j}^{3}\left(1-\mu_{i j}\right)^{3}\right)\right], i=1, \ldots, n$ e $j=1, \ldots, t$. Ressaltamos que pela Proposição 1.1 resultado (6), temos que $\mathrm{E}\left(\partial \mathrm{u}_{i j} / \partial \mu_{i j}\right)=-\phi \operatorname{Var}\left(\mathrm{u}_{i j}\right)$ e pela Proposição 1.1 resultado (3), obtemos que $\operatorname{Var}\left(\mathrm{u}_{i j}\right)=\mathrm{a}_{i j}$, com $i=1, \ldots, n$ e $j=1, \ldots, t$.

\section{A.2.2 EEGs sob heterogeneidade da dispersão}

A seguir, apresentamos algumas contas detalhadas para obtenção da equação de estimação ótima descrita em (3.10).

$$
\begin{aligned}
\Psi_{2}^{o}(\boldsymbol{\theta}) & =\sum_{i=1}^{n} \mathrm{E}\left(\frac{\partial \mathbf{b}_{i}}{\partial \boldsymbol{\theta}^{\top}}\right)^{\top} \operatorname{Cov}\left(\mathbf{b}_{i}\right)^{-1} \mathbf{b}_{i}= \\
& =\sum_{i=1}^{n} \mathrm{E}\left(\begin{array}{cc}
\frac{\partial \mathbf{b}_{1 i}}{\partial \beta^{\top}} & \frac{\partial \mathbf{b}_{1 i}}{\partial \gamma^{\top}} \\
\frac{\partial \mathbf{b}_{2 i}}{\partial \beta^{\top}} & \frac{\partial \mathbf{b}_{2 i}}{\partial \gamma^{\top}}
\end{array}\right)^{\top}\left(\begin{array}{cc}
\operatorname{Cov}\left(\mathbf{b}_{1 i}\right) & \operatorname{Cov}\left(\mathbf{b}_{1 i}, \mathbf{b}_{2 i}\right) \\
\operatorname{Cov}\left(\mathbf{b}_{2 i}, \mathbf{b}_{1 i}\right) & \operatorname{Cov}\left(\mathbf{b}_{2 i}\right)
\end{array}\right)^{-1}\left(\begin{array}{l}
\mathbf{b}_{1 i} \\
\mathbf{b}_{2 i}
\end{array}\right),
\end{aligned}
$$


em que as esperanças são descritas por

$$
\begin{aligned}
& \mathrm{E}\left(\frac{\partial \mathbf{b}_{1 i}}{\partial \boldsymbol{\beta}^{\top}}\right)^{\top}=-\phi \mathbf{X}_{i}^{\top} \mathbf{G}_{i} \mathbf{A}_{i}, \\
& \mathrm{E}\left(\frac{\partial \mathbf{b}_{1 i}}{\partial \boldsymbol{\gamma}^{\top}}\right)^{\top}=\mathrm{E}\left(\frac{\partial \mathbf{u}_{i}}{\partial \boldsymbol{\gamma}^{\top}}\right)^{\top}=\mathbf{0}, \\
& \mathrm{E}\left(\frac{\partial \mathbf{b}_{2 i}}{\partial \boldsymbol{\beta}^{\top}}\right)^{\top}=\mathrm{E}\left(\frac{\partial\left(\mathbf{d}_{i}-\boldsymbol{\phi}_{i}^{-1}\right)}{\partial \boldsymbol{\beta}^{\top}}\right)^{\top}=\mathrm{E}\left(\frac{\partial \mathbf{d}_{i}}{\partial \boldsymbol{\beta}^{\top}}\right)^{\top}=-2 \mathbf{X}_{i}^{\top} \mathbf{G}_{i} \mathrm{E}\left(\mathbf{u}_{i}\right)=\mathbf{0}, \\
& \mathrm{E}\left(\frac{\partial \mathbf{b}_{2 i}}{\partial \boldsymbol{\gamma}^{\top}}\right)^{\top}=\mathrm{E}\left(\frac{\partial\left(\mathbf{d}_{i}-\boldsymbol{\phi}_{i}^{-1}\right)}{\partial \boldsymbol{\gamma}^{\top}}\right)^{\top}=-\mathrm{E}\left(\frac{\partial \boldsymbol{\phi}_{i}^{-1}}{\partial \boldsymbol{\gamma}^{\top}}\right)^{\top}=-\mathrm{E}\left(\frac{\partial \boldsymbol{\phi}_{i}^{-1}}{\partial \boldsymbol{\delta}_{i}} \frac{\partial \boldsymbol{\delta}_{i}}{\partial \boldsymbol{\gamma}_{i}^{\top}}\right)^{\top}=-\mathbf{Q}_{i}^{\top} \mathbf{F}_{i},
\end{aligned}
$$

e as covariâncias são descritas por

$$
\begin{aligned}
\operatorname{Cov}\left(\mathbf{b}_{1 i}\right) & =\operatorname{Cov}\left(\mathbf{u}_{i}\right)=\operatorname{Var}\left(\mathbf{u}_{i}\right)^{1 / 2} \mathbf{R}\left(\mathbf{u}_{i}\right) \operatorname{Var}\left(\mathbf{u}_{i}\right)^{1 / 2}=\mathbf{A}_{i}^{1 / 2} \mathbf{R}\left(\mathbf{u}_{i}\right) \mathbf{A}_{i}^{1 / 2}, \\
\operatorname{Cov}\left(\mathbf{b}_{1 i}, \mathbf{b}_{2 i}\right) & =\operatorname{Cov}\left(\mathbf{u}_{i}, \mathbf{d}_{i}\right), \\
\operatorname{Cov}\left(\mathbf{b}_{2 i}\right) & =\operatorname{Cov}\left(\mathbf{d}_{i}\right)=\operatorname{Var}\left(\mathbf{d}_{i}\right)^{1 / 2} \mathbf{R}\left(\mathbf{d}_{i}\right) \operatorname{Var}\left(\mathbf{d}_{i}\right)^{1 / 2}=\mathbf{D}_{i}^{1 / 2} \mathbf{R}\left(\mathbf{d}_{i}\right) \mathbf{D}_{i}^{1 / 2},
\end{aligned}
$$

sendo

$\Phi_{i}=\operatorname{diag}\left(\phi_{i 1}, \ldots, \phi_{i t}\right), \quad$ com dimensão $(t \times t)$,

$\mathbf{A}_{i}=\operatorname{diag}\left(\mathrm{a}_{i 1}, \ldots, \mathrm{a}_{i t}\right), \quad$ com dimensão $(t \times t)$,

$\mathbf{Q}_{i}=\left(\mathbf{q}_{i 1}, \ldots, \mathbf{q}_{i t}\right)^{\top}, \quad$ com dimensão $(t \times q)$,

$\mathbf{F}_{i}=\operatorname{diag}\left(\partial f^{-1}\left(\delta_{i 1}\right) / \partial \delta_{i 1}, \ldots, \partial f^{-1}\left(\delta_{i t}\right) / \partial \delta_{i t}\right), \quad$ com dimensão $(t \times t) \mathrm{e}$

$\mathbf{D}_{i}=\operatorname{diag}\left(2\left(\phi_{i 1}^{-1}\right)^{2}, \ldots, 2\left(\phi_{i t}^{-1}\right)^{2}\right), \quad$ com dimensão $(t \times t)$,

$\operatorname{com~a~}_{i j}=\phi_{i j}^{-1}\left[3 \phi_{i j}^{-1} /\left(\mu_{i j}\left(1-\mu_{i j}\right)\right)+1 /\left(\mu_{i j}^{3}\left(1-\mu_{i j}\right)^{3}\right)\right], i=1, \ldots, n$ e $j=1, \ldots, t$. Ressaltamos que a matriz de variâncias de $\mathbf{d}_{i}$ é obtida pelo resultado (4) da Proposição 1.1, com $i=1, \ldots, n$.

A matriz de sensibilidade e a matriz de variabilidade para a equação de estimação generalizada descrita em (3.11) são, respectivamente, dadas por

$$
\begin{aligned}
& \sum_{i=1}^{n} \mathbf{S}_{i}=\mathrm{E}\left(\frac{\partial}{\partial \boldsymbol{\theta}^{\top}} \boldsymbol{\Psi}_{2}(\boldsymbol{\theta})\right)=\mathrm{E}\left(\begin{array}{cc}
\frac{\partial}{\partial \beta^{\top}} \boldsymbol{\Psi}_{2}(\boldsymbol{\beta}) & \frac{\partial}{\partial \gamma^{\top}} \boldsymbol{\Psi}_{2}(\boldsymbol{\beta}) \\
\frac{\partial}{\partial \beta^{\top}} \boldsymbol{\Psi}_{2}(\boldsymbol{\gamma}) & \frac{\partial}{\partial \gamma^{\top}} \boldsymbol{\Psi}_{2}(\boldsymbol{\gamma})
\end{array}\right)=
\end{aligned}
$$

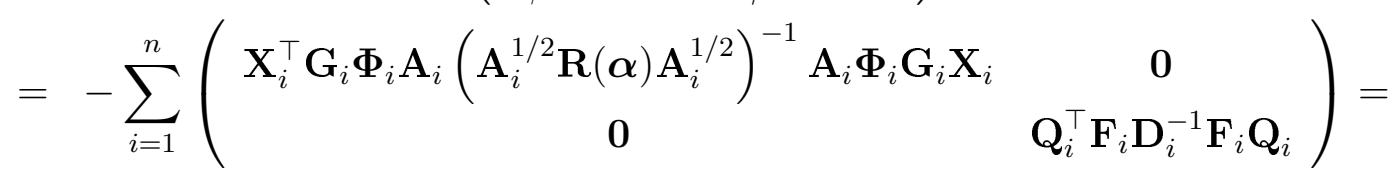

$$
\begin{aligned}
& =-\sum_{i=1}^{n} \mathbf{M}_{i}^{\top} \boldsymbol{\Lambda}_{i} \boldsymbol{\Omega}^{-1} \boldsymbol{\Lambda}_{i}^{\top} \mathbf{M}_{i}
\end{aligned}
$$


e

$$
\begin{aligned}
& \sum_{i=1}^{n} \mathbf{V}_{i}=\mathrm{E}\left(\boldsymbol{\Psi}_{2}(\boldsymbol{\theta}) \boldsymbol{\Psi}_{2}^{\top}(\boldsymbol{\theta})\right)=\mathrm{E}\left(\begin{array}{ll}
\boldsymbol{\Psi}_{2}(\boldsymbol{\beta}) \boldsymbol{\Psi}_{2}^{\top}(\boldsymbol{\beta}) & \boldsymbol{\Psi}_{2}(\boldsymbol{\beta}) \boldsymbol{\Psi}_{2}^{\top}(\boldsymbol{\gamma}) \\
\boldsymbol{\Psi}_{2}(\boldsymbol{\gamma}) \boldsymbol{\Psi}_{2}^{\top}(\boldsymbol{\beta}) & \boldsymbol{\Psi}_{2}(\boldsymbol{\gamma}) \boldsymbol{\Psi}_{2}^{\top}(\boldsymbol{\gamma})
\end{array}\right)= \\
& =\sum_{i=1}^{n}\left(\begin{array}{c}
\mathbf{X}_{i}^{\top} \mathbf{G}_{i} \boldsymbol{\Phi}_{i} \mathbf{A}_{i} \\
\mathbf{Q}_{i}^{\top} \mathbf{F}_{i}
\end{array}\right) \boldsymbol{\Omega}_{i}^{-1} \operatorname{Cov}\left(\mathbf{b}_{i}\right) \boldsymbol{\Omega}_{i}^{-1}\left(\begin{array}{cc}
\mathbf{A}_{i} \boldsymbol{\Phi}_{i} \mathbf{G}_{i} \mathbf{X}_{i} & \mathbf{F}_{i} \mathbf{Q}_{i}
\end{array}\right)= \\
& =\sum_{i=1}^{n} \mathbf{M}_{i}^{\top} \boldsymbol{\Lambda}_{i} \boldsymbol{\Omega}_{i}^{-1} \operatorname{Cov}\left(\mathbf{b}_{i}\right) \boldsymbol{\Omega}_{i}^{-1} \boldsymbol{\Lambda}_{i}^{\top} \mathbf{M}_{i},
\end{aligned}
$$

sendo $\operatorname{Cov}\left(\mathbf{b}_{i}\right)=\mathrm{E}\left(\mathbf{b}_{i} \mathbf{b}_{i}^{\top}\right)$, já que $\mathrm{E}\left(\mathbf{b}_{i}\right)=\mathbf{0}$. Ressaltamos que as matrizes de sensibilidade e variabilidade têm dimensão $(p+q) \times(p+q)$.

\section{A.3 Modelos lineares generalizados}

\section{A.3.1 EEGs sob heterogeneidade da dispersão}

A seguir, apresentamos algumas contas detalhadas para obtenção da equação de estimação ótima descrita em (4.8).

$$
\begin{aligned}
\Psi_{2}^{o}(\boldsymbol{\theta}) & =\sum_{i=1}^{n} \mathrm{E}\left(\frac{\partial \mathbf{b}_{i}}{\partial \boldsymbol{\theta}^{\top}}\right)^{\top} \operatorname{Cov}\left(\mathbf{b}_{i}\right)^{-1} \mathbf{b}_{i}= \\
& =\sum_{i=1}^{n} \mathrm{E}\left(\begin{array}{cc}
\frac{\partial \mathbf{b}_{1 i}}{\partial \beta^{\top}} & \frac{\partial \mathbf{b}_{1 i}}{\partial \gamma^{\top}} \\
\frac{\partial \mathbf{b}_{2 i}}{\partial \beta^{\top}} & \frac{\partial \mathbf{b}_{2 i}}{\partial \gamma^{\top}}
\end{array}\right)^{\top}\left(\begin{array}{cc}
\operatorname{Cov}\left(\mathbf{b}_{1 i}\right) & \operatorname{Cov}\left(\mathbf{b}_{1 i}, \mathbf{b}_{2 i}\right) \\
\operatorname{Cov}\left(\mathbf{b}_{2 i}, \mathbf{b}_{1 i}\right) & \operatorname{Cov}\left(\mathbf{b}_{2 i}\right)
\end{array}\right)^{-1}\left(\begin{array}{l}
\mathbf{b}_{1 i} \\
\mathbf{b}_{2 i}
\end{array}\right),
\end{aligned}
$$

em que as esperanças são descritas por

$$
\begin{aligned}
\mathrm{E}\left(\frac{\partial \mathbf{b}_{1 i}}{\partial \boldsymbol{\beta}^{\top}}\right)^{\top} & =-\mathbf{X}_{i}^{\top} \mathbf{G}_{i}, \\
\mathrm{E}\left(\frac{\partial \mathbf{b}_{1 i}}{\partial \boldsymbol{\gamma}^{\top}}\right)^{\top} & =\mathrm{E}\left(\frac{\partial\left[\mathbf{y}_{i}-\boldsymbol{\mu}_{i}\right]}{\partial \boldsymbol{\gamma}^{\top}}\right)^{\top}=\mathbf{0}, \\
\mathrm{E}\left(\frac{\partial \mathbf{b}_{2 i}}{\partial \boldsymbol{\beta}^{\top}}\right)^{\top} & =\mathrm{E}\left(\frac{\partial\left[\mathbf{y}_{i} \boldsymbol{\varphi}_{i}-b\left(\boldsymbol{\varphi}_{i}\right)+\dot{c}\left(\mathbf{y}_{i} ; \boldsymbol{\phi}_{i}\right)\right]}{\partial \boldsymbol{\beta}^{\top}}\right)^{\top}=\mathrm{E}\left(\frac{\partial\left[\mathbf{y}_{i} \boldsymbol{\varphi}_{i}-b\left(\boldsymbol{\varphi}_{i}\right)\right]}{\partial \boldsymbol{\beta}^{\top}}\right)^{\top} \\
\mathrm{E}\left(\frac{\partial \mathbf{b}_{2 i}}{\partial \boldsymbol{\gamma}^{\top}}\right)^{\top} & =\mathbf{X}_{i}^{\top} \mathbf{G}_{i} \mathbf{A}^{-1} \mathrm{E}\left(\mathbf{y}_{i}-\boldsymbol{\mu}_{i}\right)=\mathbf{0}, \\
& \left.=\mathrm{E}\left(\frac{\partial\left[\mathbf{y}_{i} \boldsymbol{\varphi}_{i}-b\left(\boldsymbol{\varphi}_{i}\right)+\dot{c}\left(\mathbf{y}_{i} ; \boldsymbol{\phi}_{i}\right)\right]}{\left.\partial \boldsymbol{\phi}_{i}\right)}\right)^{\top}=\mathrm{E}\left(\frac{\partial \dot{c}\left(\mathbf{y}_{i} ; \boldsymbol{\phi}_{i}\right)}{\partial \boldsymbol{\phi}_{i}^{-1}}\right)^{\top} \frac{\partial \boldsymbol{\delta}_{i}}{\partial \boldsymbol{\gamma}_{i}^{\top}}\right)^{\top}=\mathbf{Q}_{i}^{\top} \mathbf{F}_{i} \mathrm{E}\left(\ddot{\mathbf{c}}_{i}\right),
\end{aligned}
$$


e as covariâncias são descritas por

$$
\begin{aligned}
\operatorname{Cov}\left(\mathbf{b}_{1 i}\right) & =\operatorname{Var}\left(\mathbf{y}_{i}\right)^{1 / 2} \mathbf{R}\left(\mathbf{y}_{i}\right) \operatorname{Var}\left(\mathbf{y}_{i}\right)^{1 / 2}=\left(\boldsymbol{\Phi}_{i}^{-1} \mathbf{A}_{i}\right)^{1 / 2} \mathbf{R}\left(\mathbf{y}_{i}\right)\left(\mathbf{A}_{i} \boldsymbol{\Phi}_{i}^{-1}\right)^{1 / 2}, \\
\operatorname{Cov}\left(\mathbf{b}_{1 i}, \mathbf{b}_{2 i}\right) & =\operatorname{Cov}\left(\mathbf{y}_{i}, \mathbf{y}_{i} \boldsymbol{\varphi}_{i}+\dot{c}\left(\mathbf{y}_{i} ; \boldsymbol{\phi}_{i}\right)\right), \\
\operatorname{Cov}\left(\mathbf{b}_{2 i}\right) & =\operatorname{Cov}\left(\mathbf{y}_{i} \boldsymbol{\varphi}_{i}+\dot{c}\left(\mathbf{y}_{i} ; \boldsymbol{\phi}_{i}\right)\right) \cong \boldsymbol{\Pi}_{i} \operatorname{Cov}\left(\mathbf{y}_{i}\right) \boldsymbol{\Pi}_{i},
\end{aligned}
$$

sendo

$$
\begin{aligned}
\mathbf{X}_{i} & =\left(\mathbf{x}_{i 1}, \ldots, \mathbf{x}_{i t}\right)^{\top}, \quad \text { com dimensão }(t \times p), \\
\mathbf{G}_{i} & =\operatorname{diag}\left(\partial g^{-1}\left(\eta_{i 1}\right) / \partial \eta_{i 1}, \ldots, \partial g^{-1}\left(\eta_{i t}\right) / \partial \eta_{i t}\right), \quad \text { com dimensão }(t \times t), \\
\mathbf{\Phi}_{i} & =\operatorname{diag}\left(\phi_{i 1}, \ldots, \phi_{i t}\right), \quad \text { com dimensão }(t \times t), \\
\mathbf{A}_{i} & =\operatorname{diag}\left(v\left(\mu_{i 1}\right), \ldots, v\left(\mu_{i t}\right)\right), \quad \text { com dimensão }(t \times t), \\
\mathbf{Q}_{i} & =\left(\mathbf{q}_{i 1}, \ldots, \mathbf{q}_{i t}\right)^{\top}, \quad \text { com dimensão }(t \times q), \\
\mathbf{F}_{i} & =\operatorname{diag}\left(\partial f^{-1}\left(\delta_{i 1}\right) / \partial \delta_{i 1}, \ldots, \partial f^{-1}\left(\delta_{i t}\right) / \partial \delta_{i t}\right), \quad \text { com dimensão }(t \times t) \mathrm{e} \\
\ddot{\mathbf{c}}_{i} & =\operatorname{diag}\left(\ddot{c}_{i 1}, \ldots, \ddot{c}_{i t}\right), \quad \text { com dimensão }(t \times t),
\end{aligned}
$$

em que $\ddot{c}_{i j}$ é a derivada de $\dot{c}\left(\mathrm{y}_{i j} ; \phi_{i j}\right)$ com relação à $\phi_{i j}^{-1}, \operatorname{com} i=1, \ldots, n$ e $j=1, \ldots, t$.

A matriz de sensibilidade e a matriz de variabilidade para a equação de estimação generalizada descrita em (4.10) são, respectivamente, dadas por

$$
\begin{aligned}
& \sum_{i=1}^{n} \mathbf{S}_{i}=\mathrm{E}\left(\frac{\partial}{\partial \boldsymbol{\theta}^{\top}} \boldsymbol{\Psi}_{2}(\boldsymbol{\theta})\right)=\mathrm{E}\left(\begin{array}{cc}
\frac{\partial}{\partial \beta^{\top}} \boldsymbol{\Psi}_{2}(\boldsymbol{\beta}) & \frac{\partial}{\partial \gamma^{\top}} \Psi_{2}(\boldsymbol{\beta}) \\
\frac{\partial}{\partial \beta^{\top}} \boldsymbol{\Psi}_{2}(\boldsymbol{\gamma}) & \frac{\partial}{\partial \gamma^{\top}} \boldsymbol{\Psi}_{2}(\boldsymbol{\gamma})
\end{array}\right)=
\end{aligned}
$$

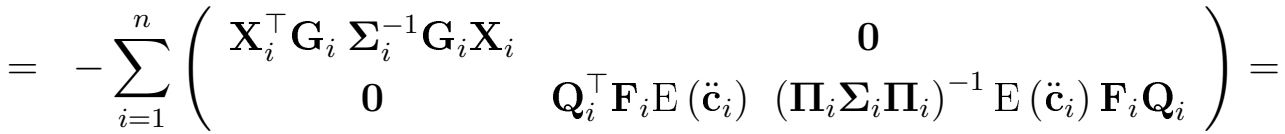

$$
\begin{aligned}
& =-\sum_{i=1}^{n} \mathbf{M}_{i}^{\top} \boldsymbol{\Lambda}_{i} \boldsymbol{\Omega}^{-1} \boldsymbol{\Lambda}_{i}^{\top} \mathbf{M}_{i}
\end{aligned}
$$

$\mathrm{e}$

$$
\begin{aligned}
& \sum_{i=1}^{n} \mathbf{V}_{i}=\mathrm{E}\left(\boldsymbol{\Psi}_{2}(\boldsymbol{\theta}) \boldsymbol{\Psi}_{2}^{\top}(\boldsymbol{\theta})\right)=\mathrm{E}\left(\begin{array}{ll}
\boldsymbol{\Psi}_{2}(\boldsymbol{\beta}) \boldsymbol{\Psi}_{2}^{\top}(\boldsymbol{\beta}) & \boldsymbol{\Psi}_{2}(\boldsymbol{\beta}) \boldsymbol{\Psi}_{2}^{\top}(\boldsymbol{\gamma}) \\
\boldsymbol{\Psi}_{2}(\boldsymbol{\gamma}) \boldsymbol{\Psi}_{2}^{\top}(\boldsymbol{\beta}) & \boldsymbol{\Psi}_{2}(\boldsymbol{\gamma}) \boldsymbol{\Psi}_{2}^{\top}(\boldsymbol{\gamma})
\end{array}\right)= \\
& =\sum_{i=1}^{n}\left(\begin{array}{c}
\mathbf{X}_{i}^{\top} \mathbf{G}_{i} \\
\mathbf{Q}_{i}^{\top} \mathbf{F}_{i} \mathrm{E}\left(\ddot{\mathbf{c}}_{i}\right)
\end{array}\right) \boldsymbol{\Omega}_{i}^{-1} \operatorname{Cov}\left(\mathbf{b}_{i}\right) \boldsymbol{\Omega}_{i}^{-1}\left(\begin{array}{ll}
\mathbf{G}_{i} \mathbf{X}_{i} & \mathrm{E}\left(\ddot{\mathbf{c}}_{i}\right) \mathbf{F}_{i} \mathbf{Q}_{i}
\end{array}\right)= \\
& =\sum_{i=1}^{n} \mathbf{M}_{i}^{\top} \boldsymbol{\Lambda}_{i} \boldsymbol{\Omega}_{i}^{-1} \operatorname{Cov}\left(\mathbf{b}_{i}\right) \boldsymbol{\Omega}_{i}^{-1} \boldsymbol{\Lambda}_{i}^{\top} \mathbf{M}_{i},
\end{aligned}
$$

sendo $\operatorname{Cov}\left(\mathbf{b}_{i}\right)=\mathrm{E}\left(\mathbf{b}_{i} \mathbf{b}_{i}^{\top}\right)$, já que $\mathrm{E}\left(\mathbf{b}_{i}\right)=\mathbf{0}$. Ressaltamos que as matrizes de sensibilidade e variabilidade têm dimensão $(p+q) \times(p+q)$. 


\section{Apêndice B}

\section{Detalhes para obtenção das medidas de influência local}

Neste apêndice, apresentamos os cálculos detalhados desenvolvidos para obtenção das medidas de influência local descritas no Capítulo 6.

\section{B.1 Influência local sob homogeneidade da dispersão}

Para modelos de regressão com suposição de homogeneidade da dispersão, temos que $\boldsymbol{\theta}=\boldsymbol{\beta}=\left(\boldsymbol{\beta}_{1}, \ldots, \beta_{p}\right)$ deixando a matriz $\boldsymbol{\Delta}$ com dimensão $p \times N$, em que $N=n t$. Assim,

$$
\boldsymbol{\Delta}=\frac{\partial \boldsymbol{\Psi}_{1}(\boldsymbol{\beta} \mid \boldsymbol{\omega})}{\partial \boldsymbol{\omega}^{\top}}=\left(\begin{array}{llll}
\frac{\partial \boldsymbol{\Psi}_{1}(\boldsymbol{\beta} \mid \boldsymbol{\omega})}{\partial \omega_{11}} & \frac{\partial \boldsymbol{\Psi}_{1}(\boldsymbol{\beta} \mid \boldsymbol{\omega})}{\partial \omega_{12}} & \ldots & \frac{\partial \boldsymbol{\Psi}_{1}(\boldsymbol{\beta} \mid \boldsymbol{\omega})}{\partial \omega_{n t}}
\end{array}\right),
$$

avaliada em $\hat{\boldsymbol{\beta}}, \hat{\phi}$ e $\boldsymbol{\omega}_{0}$. Lembrando que $\boldsymbol{\omega}=\left(\boldsymbol{\omega}_{1}^{\top}, \ldots, \boldsymbol{\omega}_{n}^{\top}\right)^{\top}, \operatorname{com} \boldsymbol{\omega}_{i}=\left(\omega_{i 1}, \ldots, \omega_{i t}\right)^{\top}$, $i=1, \ldots, n$.

\section{B.1.1 Perturbação da variável resposta}

Considerando perturbação da variável resposta conforme (6.10), a matriz $\boldsymbol{\Delta}$ definida em (B.1) pode ser expressa por $\boldsymbol{\Delta}=\mathbf{X}^{\top} \mathbf{W} \boldsymbol{\Lambda}^{-1} \mathcal{B}$, sendo

$$
\mathcal{B}=\frac{\partial \mathbf{b}_{\omega}}{\partial \boldsymbol{\omega}^{\top}}=\left(\begin{array}{llll}
\frac{\partial \mathbf{b}_{\omega}}{\partial \omega_{11}} & \frac{\partial \mathbf{b}_{\omega}}{\partial \omega_{12}} & \cdots & \frac{\partial \mathbf{b}_{\omega}}{\partial \omega_{n t}}
\end{array}\right)
$$

avaliada em $\hat{\boldsymbol{\beta}}, \hat{\phi}$ e $\boldsymbol{\omega}_{0}$. 
A seguir, apresentamos detalhadamente a obtenção da matriz $\mathcal{B}$ para as equações (2.4), (3.3) e (4.3) definidas, respectivamente, para modelos de regressão beta, modelos de regressão simplex e modelos lineares generalizados.

BETA

De (2.4), temos que a $j$-ésima observação do vetor perturbado na variável resposta referente à $i$-ésima unidade experimental, $\mathrm{b}_{\omega i j}$, é dada por

$$
\mathrm{b}_{\omega i j}=\mathrm{y}_{\omega i j}^{*}-\mu_{i j}^{*}
$$

em que

$$
\mathrm{y}_{\omega i j}^{*}=\log \left(\frac{\mathrm{y}_{\omega i j}}{1-\mathrm{y}_{\omega i j}}\right)=\log \left(\frac{\mathrm{y}_{i j}+\omega_{i j} s_{i j}}{1-\mathrm{y}_{i j}-\omega_{i j} s_{i j}}\right),
$$

sendo $s_{i j}=\sqrt{v\left(\mu_{i j}\right) /(1+\phi)}$ o desvio padrão de uma variável resposta $\mathrm{y}_{i j}$ conforme equação (1.3), $i=1, \ldots, n$ e $j=1, \ldots, t$. Assim, a derivada de (B.1) com relação à perturbação $\omega_{i j}$ é dada por

$$
\frac{\partial \mathrm{b}_{\omega i j}}{\partial \omega_{i j}}=\frac{\partial \mathrm{y}_{\omega i j}^{*}}{\partial \omega_{i j}}=\frac{\left(1-\mathrm{y}_{\omega i j}\right)}{\mathrm{y}_{\omega i j}}\left[\frac{s_{i j}\left(1-\mathrm{y}_{\omega i j}\right)+s_{i j} \mathrm{y}_{\omega i j}}{\left(1-\mathrm{y}_{\omega i j}\right)^{2}}\right]=\frac{s_{i j}}{v\left(\mathrm{y}_{\omega i j}\right)},
$$

sendo $v\left(\mathrm{y}_{\omega i j}\right)=\mathrm{y}_{\omega i j}\left(1-\mathrm{y}_{\omega i j}\right), \operatorname{com} i=1, \ldots, n$ e $j=1, \ldots, t$. Notemos que

$$
\frac{\partial \mathrm{b}_{\omega i j}}{\partial \omega_{i j^{\prime}}}=0
$$

se $j \neq j^{\prime}, \operatorname{com} j^{\prime}=1, \ldots, t$.

\section{Simplex}

Seja $s_{i j}=\sqrt{\operatorname{Var}\left(\mathrm{y}_{i j}\right)}$, então a derivada da equação (6.13) com relação à perturbação $\omega_{i j}$ é, detalhadamente, dada por

$$
\begin{aligned}
\frac{\partial \mathrm{b}_{\omega i j}}{\partial \omega_{i j}}= & -\frac{1}{2 \mu_{i j}\left(1-\mu_{i j}\right)} \frac{\partial}{\partial \omega_{i j}}\left\{\left(\mathrm{y}_{\omega i j}-\mu_{i j}\right)\left[d\left(\mathrm{y}_{\omega i j} ; \mu_{i j}\right)+\frac{1}{\mu_{i j}^{2}\left(1-\mu_{i j}\right)^{2}}\right]\right\} \\
= & -\frac{1}{2 \mu_{i j}\left(1-\mu_{i j}\right)}\left\{s_{i j}\left[d\left(\mathrm{y}_{\omega i j} ; \mu_{i j}\right)+\frac{1}{\mu_{i j}^{2}\left(1-\mu_{i j}\right)^{2}}\right]+\left(\mathrm{y}_{\omega i j}-\mu_{i j}\right) \frac{\partial d\left(\mathrm{y}_{\omega i j} ; \mu_{i j}\right)}{\partial \omega_{i j}}\right\} \\
= & -\frac{s_{i j}}{2 \mu_{i j}\left(1-\mu_{i j}\right)}\left\{d\left(\mathrm{y}_{\omega i j} ; \mu_{i j}\right)+\frac{1}{\mu_{i j}^{2}\left(1-\mu_{i j}\right)^{2}}+\right. \\
& \left.+\frac{\left(\mathrm{y}_{\omega i j}-\mu_{i j}\right)^{2}}{\mu_{i j}^{2}\left(1-\mu_{i j}\right)^{2} \mathrm{y}_{\omega i j}^{2}\left(1-\mathrm{y}_{\omega i j}\right)^{2}}\left[\mathrm{y}_{\omega i j}-2 \mathrm{y}_{\omega i j} \mu_{i j}+\mu_{i j}\right]\right\}=s_{i j} \xi_{i j},
\end{aligned}
$$

sendo $\xi_{i j}$ escrito conforme (6.15), com $i=1, \ldots, n$ e $j=1, \ldots, t$. Ressaltamos que $s_{i j}$ é dado pela raiz quadrada da variância definida em (1.9), já que aqui a variável resposta $\mathrm{y}_{i j}$ tem distribuição simplex com parâmetros $\mu_{i j}$ e $\phi$, com $i=1, \ldots, n$ e $j=1, \ldots, t$. 


\section{B.1.2 Perturbação individual de covariáveis}

BETA

De (6.24), as derivadas de $\boldsymbol{\Lambda}_{\omega i}, \boldsymbol{\Omega}_{\omega i}$ e $\mathbf{b}_{\omega i}$ todas com relação à $\boldsymbol{\omega}_{i}^{\top}$ são dadas por

$$
\begin{aligned}
\frac{\partial \boldsymbol{\Lambda}_{\omega i}}{\partial \boldsymbol{\omega}_{i}^{\top}} & =\frac{\partial\left[\phi \mathbf{G}_{\omega i} \mathbf{A}_{\omega i}\right]}{\partial \boldsymbol{\omega}_{i}^{\top}}=\phi \mathbf{G}_{\omega i} \frac{\partial \mathbf{A}_{\omega i}}{\partial \boldsymbol{\omega}_{i}^{\top}}+\phi \frac{\partial \mathbf{G}_{\omega i}}{\partial \boldsymbol{\omega}_{i}^{\top}} \mathbf{A}_{\omega i}, \\
\frac{\partial \boldsymbol{\Omega}_{\omega i}}{\partial \boldsymbol{\omega}_{i}^{\top}} & =\frac{\partial\left[\mathbf{A}_{\omega i}^{1 / 2} \mathbf{R}(\boldsymbol{\alpha}) \mathbf{A}_{\omega i}^{1 / 2}\right]}{\partial \boldsymbol{\omega}_{i}^{\top}}=\mathbf{A}_{\omega i}^{1 / 2} \mathbf{R}(\boldsymbol{\alpha}) \frac{\partial \mathbf{A}_{\omega i}^{1 / 2}}{\partial \boldsymbol{\omega}_{i}^{\top}}+\frac{\partial \mathbf{A}_{\omega i}^{1 / 2}}{\partial \boldsymbol{\omega}_{i}^{\top}} \mathbf{R}(\boldsymbol{\alpha}) \mathbf{A}_{\omega i}^{1 / 2}, \quad \mathrm{e} \\
\frac{\partial \mathbf{b}_{\omega i}}{\partial \boldsymbol{\omega}_{i}^{\top}} & =\frac{\partial\left[\mathbf{y}_{i}^{*}-\boldsymbol{\mu}_{\omega i}^{*}\right]}{\partial \boldsymbol{\omega}_{i}^{\top}}=-\frac{\partial \boldsymbol{\mu}_{\omega i}^{*}}{\partial \boldsymbol{\omega}_{i}^{\top}},
\end{aligned}
$$

sendo

$$
\begin{aligned}
& \frac{\partial \mathbf{A}_{\omega i}}{\partial \boldsymbol{\omega}_{i}^{\top}}=\operatorname{diag}\left(\frac{\partial \mathrm{a}_{\omega i 1}}{\partial \omega_{i 1}}, \ldots, \frac{\partial \mathrm{a}_{\omega i t}}{\partial \omega_{i t}}\right), \\
& \frac{\partial \mathbf{G}_{\omega i}}{\partial \boldsymbol{\omega}_{i}^{\top}}=\operatorname{diag}\left(\frac{\partial}{\partial \omega_{i 1}}\left[\frac{\partial g^{-1}\left(\eta_{\omega i 1}\right)}{\partial \eta_{\omega i 1}}\right], \ldots, \frac{\partial}{\partial \omega_{i t}}\left[\frac{\partial g^{-1}\left(\eta_{\omega i t}\right)}{\partial \eta_{\omega i t}}\right]\right), \quad \mathrm{e} \\
& \frac{\partial \boldsymbol{\mu}_{\omega i}^{*}}{\partial \boldsymbol{\omega}_{i}^{\top}}=\operatorname{diag}\left(\frac{\partial \mu_{\omega i 1}^{*}}{\partial \omega_{i 1}}, \ldots, \frac{\partial \mu_{\omega i t}^{*}}{\partial \omega_{i t}}\right),
\end{aligned}
$$

em que

$$
\begin{aligned}
& \frac{\partial \mathrm{a}_{\omega i j}}{\partial \omega_{i j}}=\mathcal{E}_{1 \omega i j} \frac{\partial g^{-1}\left(\eta_{\omega i j}\right)}{\partial \eta_{\omega i j}} \beta_{k} s_{x_{k}}, \\
& \frac{\partial}{\partial \omega_{i j}}\left[\frac{\partial g^{-1}\left(\eta_{\omega i j}\right)}{\partial \eta_{\omega i j}}\right]=\frac{\partial^{2} g^{-1}\left(\eta_{\omega i j}\right)}{\partial \eta_{\omega i j}^{2}} \beta_{k} s_{x_{k}}, \\
& \frac{\partial \mathrm{a}_{\omega i j}^{1 / 2}}{\partial \omega_{i j}}=\frac{1}{2} \mathrm{a}_{\omega i j}^{-1 / 2} \frac{\partial \mathrm{a}_{\omega i j}}{\partial \omega_{i j}}, \quad \mathrm{e} \\
& \frac{\partial \mu_{\omega i j}^{*}}{\partial \omega_{i j}}=\phi \mathbf{a}_{\omega i j} \frac{\partial g^{-1}\left(\eta_{\omega i j}\right)}{\partial \eta_{\omega i j}} \beta_{k} s_{x_{k}},
\end{aligned}
$$

com $\mathcal{E}_{1 \omega i j}$ definido em $(6.25), i=1, \ldots, n, j=1, \ldots, t$ e $k$ representa a coluna de $\mathbf{X}$ com perturbação, $k=2, \ldots, p$.

\section{Simplex}

De (6.26), as derivadas de $\boldsymbol{\Lambda}_{\omega i}, \boldsymbol{\Omega}_{\omega i}$ e $\mathbf{b}_{\omega i}$ todas com relação à $\boldsymbol{\omega}_{i}^{\top}$ são dadas por

$$
\begin{aligned}
& \frac{\partial \boldsymbol{\Lambda}_{\omega i}}{\partial \boldsymbol{\omega}_{i}^{\top}}=\frac{\partial\left[\phi \mathbf{G}_{\omega i} \mathbf{A}_{\omega i}\right]}{\partial \boldsymbol{\omega}_{i}^{\top}}=\phi \mathbf{G}_{\omega i} \frac{\partial \mathbf{A}_{\omega i}}{\partial \boldsymbol{\omega}_{i}^{\top}}+\phi \frac{\partial \mathbf{G}_{\omega i}}{\partial \boldsymbol{\omega}_{i}^{\top}} \mathbf{A}_{\omega i} \\
& \frac{\partial \boldsymbol{\Omega}_{\omega i}}{\partial \boldsymbol{\omega}_{i}^{\top}}=\frac{\partial\left[\mathbf{A}_{\omega i}^{1 / 2} \mathbf{R}(\boldsymbol{\alpha}) \mathbf{A}_{\omega i}^{1 / 2}\right]}{\partial \boldsymbol{\omega}_{i}^{\top}}=\mathbf{A}_{\omega i}^{1 / 2} \mathbf{R}(\boldsymbol{\alpha}) \frac{\partial \mathbf{A}_{\omega i}^{1 / 2}}{\partial \boldsymbol{\omega}_{i}^{\top}}+\frac{\partial \mathbf{A}_{\omega i}^{1 / 2}}{\partial \boldsymbol{\omega}_{i}^{\top}} \mathbf{R}(\boldsymbol{\alpha}) \mathbf{A}_{\omega i}^{1 / 2}, \quad \mathrm{e} \\
& \frac{\partial \mathbf{b}_{\omega i}}{\partial \boldsymbol{\omega}_{i}^{\top}}=\frac{\partial \mathbf{u}_{\omega i}}{\partial \boldsymbol{\omega}_{i}^{\top}}
\end{aligned}
$$


sendo

em que

$$
\begin{aligned}
& \frac{\partial \mathbf{A}_{\omega i}}{\partial \boldsymbol{\omega}_{i}^{\top}}=\operatorname{diag}\left(\frac{\partial \mathrm{a}_{\omega i 1}}{\partial \omega_{i 1}}, \ldots, \frac{\partial \mathrm{a}_{\omega i t}}{\partial \omega_{i t}}\right), \\
& \frac{\partial \mathbf{G}_{\omega i}}{\partial \boldsymbol{\omega}_{i}^{\top}}=\operatorname{diag}\left(\frac{\partial}{\partial \omega_{i 1}}\left[\frac{\partial g^{-1}\left(\eta_{\omega i 1}\right)}{\partial \eta_{\omega i 1}}\right], \ldots, \frac{\partial}{\partial \omega_{i t}}\left[\frac{\partial g^{-1}\left(\eta_{\omega i t}\right)}{\partial \eta_{\omega i t}}\right]\right), \quad \mathrm{e} \\
& \frac{\partial \mathbf{u}_{\omega i}}{\partial \boldsymbol{\omega}_{i}^{\top}}=\operatorname{diag}\left(\frac{\partial \mathbf{u}_{\omega i 1}}{\partial \omega_{i 1}}, \ldots, \frac{\partial \mathbf{u}_{\omega i t}}{\partial \omega_{i t}}\right),
\end{aligned}
$$

$$
\begin{array}{rlrl}
\frac{\partial \mathbf{a}_{\omega i j}}{\partial \omega_{i j}} & =\mathcal{E}_{1 \omega i j} \frac{\partial g^{-1}\left(\eta_{\omega i j}\right)}{\partial \eta_{\omega i j}} \beta_{k} s_{x_{k}}, & & \frac{\partial}{\partial \omega_{i j}}\left[\frac{\partial g^{-1}\left(\eta_{\omega i j}\right)}{\partial \eta_{\omega i j}}\right]=\frac{\partial^{2} g^{-1}\left(\eta_{\omega i j}\right)}{\partial \eta_{\omega i j}^{2}} \beta_{k} s_{x_{k}}, \\
\frac{\partial \mathbf{a}_{\omega i j}^{1 / 2}}{\partial \omega_{i j}}=\frac{1}{2} \mathbf{a}_{\omega i j}^{-1 / 2} \frac{\partial \mathbf{a}_{\omega i j}}{\partial \omega_{i j}}, & \text { e } & \frac{\partial \mathbf{u}_{\omega i j}}{\partial \omega_{i j}}=\mathcal{U}_{\omega i j} \frac{\partial g^{-1}\left(\eta_{\omega i j}\right)}{\partial \eta_{\omega i j}} \beta_{k} s_{x_{k}},
\end{array}
$$

com $\mathcal{E}_{1 \omega i j}$ e $\mathcal{U}_{\omega i j}$ definidos, respectivamente, em (6.28) e $(6.29), i=1, \ldots, n, j=1, \ldots, t$ e $k$ representa a coluna de $\mathbf{X}$ com perturbação, $k=2, \ldots, p$. Ressaltamos que $\mathcal{E}_{1 \omega i j}=$ $\partial \mathrm{a}_{\omega i j} / \partial \mu_{\omega i j}$ e $\mathcal{U}_{\omega i j}=\partial \mathrm{u}_{\omega i j} / \partial \mu_{\omega i j}$.

\section{B.1.3 Perturbação no parâmetro de precisão}

BETA

De (6.32), as derivadas de $\boldsymbol{\Lambda}_{\omega i}, \boldsymbol{\Omega}_{\omega i}$ e $\mathbf{b}_{\omega i}$ todas com relação à $\boldsymbol{\omega}_{i}^{\top}$ são dadas por

$$
\begin{aligned}
& \frac{\partial \boldsymbol{\Lambda}_{\omega i}}{\partial \boldsymbol{\omega}_{i}^{\top}}=\frac{\partial\left[\mathbf{G}_{i} \boldsymbol{\Phi}_{\omega i} \mathbf{A}_{\omega i}\right]}{\partial \boldsymbol{\omega}_{i}^{\top}}=\mathbf{G}_{i} \boldsymbol{\Phi}_{\omega i} \frac{\partial \mathbf{A}_{\omega i}}{\partial \boldsymbol{\omega}_{i}^{\top}}+\mathbf{G}_{i} \frac{\partial \boldsymbol{\Phi}_{\omega i}}{\partial \boldsymbol{\omega}_{i}^{\top}} \mathbf{A}_{\omega i}, \\
& \frac{\partial \boldsymbol{\Omega}_{\omega i}}{\partial \boldsymbol{\omega}_{i}^{\top}}=\frac{\partial\left[\mathbf{A}_{\omega i}^{1 / 2} \mathbf{R}(\boldsymbol{\alpha}) \mathbf{A}_{\omega i}^{1 / 2}\right]}{\partial \boldsymbol{\omega}_{i}^{\top}}=\mathbf{A}_{\omega i}^{1 / 2} \mathbf{R}(\boldsymbol{\alpha}) \frac{\partial \mathbf{A}_{\omega i}^{1 / 2}}{\partial \boldsymbol{\omega}_{i}^{\top}}+\frac{\partial \mathbf{A}_{\omega i}^{1 / 2}}{\partial \boldsymbol{\omega}_{i}^{\top}} \mathbf{R}(\boldsymbol{\alpha}) \mathbf{A}_{\omega i}^{1 / 2}, \quad \mathrm{e} \\
& \frac{\partial \mathbf{b}_{\omega i}}{\partial \boldsymbol{\omega}_{i}^{\top}}=\frac{\partial\left[\mathbf{y}_{i}^{*}-\boldsymbol{\mu}_{\omega i}^{*}\right]}{\partial \boldsymbol{\omega}_{i}^{\top}}=-\frac{\partial \boldsymbol{\mu}_{\omega i}^{*}}{\partial \boldsymbol{\omega}_{i}^{\top}},
\end{aligned}
$$

sendo

em que

$$
\begin{aligned}
& \frac{\partial \boldsymbol{\Phi}_{\omega i}}{\partial \boldsymbol{\omega}_{i}^{\top}}=\operatorname{diag}\left(\frac{\partial \phi_{\omega i 1}}{\partial \omega_{i 1}}, \ldots, \frac{\partial \phi_{\omega i t}}{\partial \omega_{i t}}\right), \\
& \frac{\partial \mathbf{A}_{\omega i}}{\partial \boldsymbol{\omega}_{i}^{\top}}=\operatorname{diag}\left(\frac{\partial \mathrm{a}_{\omega i 1}}{\partial \omega_{i 1}}, \ldots, \frac{\partial \mathrm{a}_{\omega i t}}{\partial \omega_{i t}}\right), \quad \mathrm{e} \\
& \frac{\partial \boldsymbol{\mu}_{\omega i}^{*}}{\partial \boldsymbol{\omega}_{i}^{\top}}=\operatorname{diag}\left(\frac{\partial \mu_{\omega i 1}^{*}}{\partial \omega_{i 1}}, \ldots, \frac{\partial \mu_{\omega i t}^{*}}{\partial \omega_{i t}}\right),
\end{aligned}
$$

$$
\begin{aligned}
\frac{\partial \phi_{\omega i j}}{\partial \omega_{i j}} & =-\frac{\phi}{\omega_{i j}^{2}}, & \frac{\partial \mathrm{a}_{\omega i j}}{\partial \omega_{i j}} & =-\frac{\phi}{\omega_{i j}^{2}} \mathcal{E}_{2 \omega i j}, \\
\frac{\partial \mathbf{a}_{\omega i j}^{1 / 2}}{\partial \omega_{i j}} & =\frac{1}{2} \mathbf{a}_{\omega i j}^{-1 / 2} \frac{\partial \mathrm{a}_{\omega i j}}{\partial \omega_{i j}}, & \text { e } & \frac{\partial \mu_{\omega i j}^{*}}{\partial \omega_{i j}}=-\frac{\phi}{\omega_{i j}^{2}} \mathcal{E}_{3 \omega i j},
\end{aligned}
$$


com $\mathcal{E}_{2 \omega i j}$ e $\mathcal{E}_{3 \omega i j}$ definidos, respectivamente, (6.33) e $(6.34), i=1, \ldots, n$ e $j=1, \ldots, t$.

\section{SIMPLEX}

De (6.35), as derivadas de $\boldsymbol{\Lambda}_{\omega i}$ e $\boldsymbol{\Omega}_{\omega i}$ ambas com relação à $\boldsymbol{\omega}_{i}^{\top}$ são dadas por

$$
\begin{aligned}
& \frac{\partial \boldsymbol{\Lambda}_{\omega i}}{\partial \boldsymbol{\omega}_{i}^{\top}}=\frac{\partial\left[\mathbf{G}_{i} \boldsymbol{\Phi}_{\omega i} \mathbf{A}_{\omega i}\right]}{\partial \boldsymbol{\omega}_{i}^{\top}}=\mathbf{G}_{i} \boldsymbol{\Phi}_{\omega i} \frac{\partial \mathbf{A}_{\omega i}}{\partial \boldsymbol{\omega}_{i}^{\top}}+\mathbf{G}_{i} \frac{\partial \boldsymbol{\Phi}_{\omega i}}{\partial \boldsymbol{\omega}_{i}^{\top}} \mathbf{A}_{\omega i} \quad \text { e } \\
& \frac{\partial \boldsymbol{\Omega}_{\omega i}}{\partial \boldsymbol{\omega}_{i}^{\top}}=\frac{\partial\left[\mathbf{A}_{\omega i}^{1 / 2} \mathbf{R}(\boldsymbol{\alpha}) \mathbf{A}_{\omega i}^{1 / 2}\right]}{\partial \boldsymbol{\omega}_{i}^{\top}}=\mathbf{A}_{\omega i}^{1 / 2} \mathbf{R}(\boldsymbol{\alpha}) \frac{\partial \mathbf{A}_{\omega i}^{1 / 2}}{\partial \boldsymbol{\omega}_{i}^{\top}}+\frac{\partial \mathbf{A}_{\omega i}^{1 / 2}}{\partial \boldsymbol{\omega}_{i}^{\top}} \mathbf{R}(\boldsymbol{\alpha}) \mathbf{A}_{\omega i}^{1 / 2},
\end{aligned}
$$

sendo

$$
\frac{\partial \boldsymbol{\Phi}_{\omega i}}{\partial \boldsymbol{\omega}_{i}^{\top}}=\operatorname{diag}\left(\frac{\partial \phi_{\omega i 1}}{\partial \omega_{i 1}}, \ldots, \frac{\partial \phi_{\omega i t}}{\partial \omega_{i t}}\right) \quad \text { e } \quad \frac{\partial \mathbf{A}_{\omega i}}{\partial \boldsymbol{\omega}_{i}^{\top}}=\operatorname{diag}\left(\frac{\partial \mathrm{a}_{\omega i 1}}{\partial \omega_{i 1}}, \ldots, \frac{\partial \mathrm{a}_{\omega i t}}{\partial \omega_{i t}}\right)
$$

em que

$$
\frac{\partial \phi_{\omega i j}}{\partial \omega_{i j}}=-\frac{\phi}{\omega_{i j}^{2}}, \quad \frac{\partial \mathrm{a}_{\omega i j}}{\partial \omega_{i j}}=-\frac{\phi}{\omega_{i j}^{2}} \mathcal{E}_{2 \omega i j}, \quad \text { e } \quad \frac{\partial \mathrm{a}_{\omega i j}^{1 / 2}}{\partial \omega_{i j}}=\frac{1}{2} \mathrm{a}_{\omega i j}^{-1 / 2} \frac{\partial \mathbf{a}_{\omega i j}}{\partial \omega_{i j}},
$$

com $\mathcal{E}_{2 \omega i j}$ definido (6.36), $i=1, \ldots, n$ e $j=1, \ldots, t$.

\section{B.2 Influência local sob heterogeneidade da dispersão}

A seguir, apresentamos contas detalhadas para algumas expressões definidas na Seção 6.4.3.

\section{B.2.1 Perturbação individual de covariáveis}

\section{B.2.1.1 Matriz Q totalmente diferente da matriz $\mathrm{X}$}

BETA

De (6.44), as derivadas de $\boldsymbol{\Lambda}_{\omega i}, \boldsymbol{\Omega}_{\omega i}$ e $\mathbf{b}_{\omega i}$ todas com relação à $\boldsymbol{\omega}_{i}^{\top}$ são dadas por

$$
\begin{aligned}
& \frac{\partial \boldsymbol{\Lambda}_{\omega i}}{\partial \boldsymbol{\omega}_{i}^{\top}}=\left(\begin{array}{c}
\frac{\partial\left[\mathbf{G}_{\omega i} \mathbf{\Phi}_{i} \mathbf{A}_{\omega i}\right]}{\partial \boldsymbol{\omega}_{i}^{\top}} \\
\frac{\partial\left[\mathbf{F}_{i} \mathbf{C}_{\omega i}\right]}{\partial \boldsymbol{\omega}_{i}^{\top}}
\end{array}\right)=\left(\begin{array}{c}
\mathbf{G}_{\omega i} \boldsymbol{\Phi}_{i} \frac{\partial \mathbf{A}_{\omega i}}{\partial \boldsymbol{\omega}_{i}^{\top}}+\frac{\partial \mathbf{G}_{\omega i}}{\partial \boldsymbol{\omega}_{i}^{\top}} \mathbf{\Phi}_{i} \mathbf{A}_{\omega i} \\
\mathbf{F}_{i} \frac{\partial \mathbf{C}_{\omega i}}{\partial \boldsymbol{\omega}_{i}^{\top}}
\end{array}\right), \\
& \frac{\partial \boldsymbol{\Omega}_{\omega i}}{\partial \boldsymbol{\omega}_{i}^{\top}}=\frac{\partial\left[\mathbf{A}_{\omega i}^{1 / 2} \mathbf{R}(\boldsymbol{\alpha}) \mathbf{A}_{\omega i}^{1 / 2}\right]}{\partial \boldsymbol{\omega}_{i}^{\top}}=\mathbf{A}_{\omega i}^{1 / 2} \mathbf{R}(\boldsymbol{\alpha}) \frac{\partial \mathbf{A}_{\omega i}^{1 / 2}}{\partial \boldsymbol{\omega}_{i}^{\top}}+\frac{\partial \mathbf{A}_{\omega i}^{1 / 2}}{\partial \boldsymbol{\omega}_{i}^{\top}} \mathbf{R}(\boldsymbol{\alpha}) \mathbf{A}_{\omega i}^{1 / 2}, \quad \mathrm{e} \\
& \frac{\partial \mathbf{b}_{\omega i}}{\partial \boldsymbol{\omega}_{i}^{\top}}=\frac{\partial\left[\mathbf{y}_{i}^{*}-\boldsymbol{\mu}_{\omega i}^{*}\right]}{\partial \boldsymbol{\omega}_{i}^{\top}}=-\frac{\partial \boldsymbol{\mu}_{\omega i}^{*}}{\partial \boldsymbol{\omega}_{i}^{\top}}
\end{aligned}
$$


sendo as expressões das derivadas de $\mathbf{A}_{\omega i}, \mathbf{G}_{\omega i}$ e $\boldsymbol{\mu}_{\omega i}^{*}$ com relação à $\boldsymbol{\omega}_{i}^{\top}$ dadas na Seção B.1.2 do Apêndice B e $\partial \mathbf{C}_{\omega i} / \partial \boldsymbol{\omega}_{i}^{\top}=\operatorname{diag}\left(\partial \mathrm{c}_{\omega i 1} / \partial \omega_{i 1}, \ldots, \partial \mathrm{c}_{\omega i t} / \partial \omega_{i t}\right)$, em que

$$
\begin{aligned}
& \frac{\partial \mathrm{a}_{\omega i j}}{\partial \omega_{i j}}=\mathcal{E}_{1 \omega i j} \frac{\partial g^{-1}\left(\eta_{\omega i j}\right)}{\partial \eta_{\omega i j}} \beta_{k} s_{x_{k}}, \\
& \frac{\partial}{\partial \omega_{i j}}\left[\frac{\partial g^{-1}\left(\eta_{\omega i j}\right)}{\partial \eta_{\omega i j}}\right]=\frac{\partial^{2} g^{-1}\left(\eta_{\omega i j}\right)}{\partial \eta_{\omega i j}^{2}} \beta_{k} s_{x_{k}}, \quad \frac{\partial \mathrm{c}_{\omega i j}}{\partial \omega_{i j}}=\left[\mathrm{a}_{\omega i j}+\phi_{i j} \mathcal{E}_{2 \omega i j}\right] \frac{\partial g^{-1}\left(\eta_{\omega i j}\right)}{\partial \eta_{\omega i j}} \beta_{k} s_{x_{k}}, \\
& \frac{\partial \mathbf{a}_{\omega i j}^{1 / 2}}{\partial \omega_{i j}}=\frac{1}{2} \mathbf{a}_{\omega i j}^{-1 / 2} \frac{\partial \mathbf{a}_{\omega i j}}{\partial \omega_{i j}}, \quad \quad \text { e } \quad \frac{\partial \mu_{\omega i j}^{*}}{\partial \omega_{i j}}=\phi_{i j} \mathrm{a}_{\omega i j} \frac{\partial g^{-1}\left(\eta_{\omega i j}\right)}{\partial \eta_{\omega i j}} \beta_{k} s_{x_{k}} \text {, }
\end{aligned}
$$

$\operatorname{com} \mathcal{E}_{1 \omega i j}$ e $\mathcal{E}_{2 \omega i j}$ definidos em $(6.45)$ e $(6.46), i=1, \ldots, n, j=1, \ldots, t$ e $k$ representa a $k$-ésima coluna de $\mathbf{X}$ com perturbação, $k=2, \ldots, p$.

\section{SIMPLEX}

De (6.47), as derivadas de $\boldsymbol{\Lambda}_{\omega i}, \boldsymbol{\Omega}_{\omega i}$ e $\mathbf{b}_{\omega i}$ todas com relação à $\boldsymbol{\omega}_{i}^{\top}$ são dadas por

$$
\begin{aligned}
\frac{\partial \boldsymbol{\Lambda}_{\omega i}}{\partial \boldsymbol{\omega}_{i}^{\top}} & =\left(\begin{array}{cc}
\frac{\partial\left[\mathbf{G}_{\omega i} \boldsymbol{\Phi}_{i} \mathbf{A}_{\omega i}\right]}{\partial \boldsymbol{\omega}_{i}^{\top}} & \mathbf{0} \\
\mathbf{0} & \frac{\partial \mathbf{F}_{i}}{\partial \boldsymbol{\omega}_{i}^{\top}}
\end{array}\right)=\left(\begin{array}{cc}
\mathbf{G}_{\omega i} \boldsymbol{\Phi}_{i} \frac{\partial \mathbf{A}_{\omega i}}{\partial \boldsymbol{\omega}_{i}^{\top}}+\frac{\partial \mathbf{G}_{\omega i}}{\partial \boldsymbol{\omega}_{i}^{\top}} \boldsymbol{\Phi}_{i} \mathbf{A}_{\omega i} & \mathbf{0} \\
\mathbf{0} & \mathbf{0}
\end{array}\right), \\
\frac{\partial \boldsymbol{\Omega}_{\omega i}}{\partial \boldsymbol{\omega}_{i}^{\top}} & =\left(\begin{array}{cc}
\frac{\partial\left[\mathbf{A}_{\omega i}^{1 / 2} \mathbf{R}(\boldsymbol{\alpha}) \mathbf{A}_{\omega i}^{1 / 2}\right]}{\partial \boldsymbol{\omega}_{i}^{\top}} & \mathbf{0} \\
\mathbf{0} & \frac{\partial \mathbf{D}_{i}}{\partial \boldsymbol{\omega}_{i}^{i}}
\end{array}\right)=\left(\begin{array}{ccc}
\mathbf{A}_{\omega i}^{1 / 2} \mathbf{R}(\boldsymbol{\alpha}) \frac{\partial \mathbf{A}_{\omega i}^{1 / 2}}{\partial \boldsymbol{\omega}_{i}^{\top}}+\frac{\partial \mathbf{A}_{\omega i}^{1 / 2}}{\partial \boldsymbol{\omega}_{i}^{\top}} \mathbf{R}(\boldsymbol{\alpha}) \mathbf{A}_{\omega i}^{1 / 2} & \mathbf{0} \\
\mathbf{0} & \mathbf{0}
\end{array}\right),
\end{aligned}
$$

e

$$
\frac{\partial \mathbf{b}_{\omega i}}{\partial \boldsymbol{\omega}_{i}^{\top}}=\left(\begin{array}{c}
\frac{\partial \mathbf{u}_{\omega i}}{\partial \boldsymbol{\omega}_{i}^{\top}} \\
\frac{\partial \mathbf{b}_{2 \omega i}}{\partial \boldsymbol{\omega}_{i}^{\top}}
\end{array}\right),
$$

sendo as expressões das derivadas de $\mathbf{A}_{\omega i}, \mathbf{G}_{\omega i}$ e $\mathbf{u}_{\omega i}$ com relação à $\boldsymbol{\omega}_{i}^{\top}$ dadas na Seção B.1.2 do Apêndice B e $\partial \mathbf{b}_{2 \omega i} / \partial \boldsymbol{\omega}_{i}^{\top}=\operatorname{diag}\left(\partial \mathrm{b}_{2 \omega i 1} / \partial \omega_{i 1}, \ldots, \partial \mathrm{b}_{2 \omega i t} / \partial \omega_{i t}\right)$, em que

$$
\begin{array}{rlrl}
\frac{\partial \mathrm{a}_{\omega i j}}{\partial \omega_{i j}} & =\mathcal{E}_{1 \omega i j} \frac{\partial g^{-1}\left(\eta_{\omega i j}\right)}{\partial \eta_{\omega i j}} \beta_{k} s_{x_{k}}, & & \frac{\partial}{\partial \omega_{i j}}\left[\frac{\partial g^{-1}\left(\eta_{\omega i j}\right)}{\partial \eta_{\omega i j}}\right]=\frac{\partial^{2} g^{-1}\left(\eta_{\omega i j}\right)}{\partial \eta_{\omega i j}^{2}} \beta_{k} s_{x_{k}}, \\
\frac{\partial \mathrm{a}_{\omega i j}^{1 / 2}}{\partial \omega_{i j}}=\frac{1}{2} \mathrm{a}_{\omega i j}^{-1 / 2} \frac{\partial \mathrm{a}_{\omega i j}}{\partial \omega_{i j}}, & \frac{\partial \mathrm{u}_{\omega i j}}{\partial \omega_{i j}}=\mathcal{U}_{\omega i j} \frac{\partial g^{-1}\left(\eta_{\omega i j}\right)}{\partial \eta_{\omega i j}} \beta_{k} s_{x_{k}}, \quad \mathrm{e} \\
\frac{\partial \mathrm{b}_{2 \omega i j}}{\partial \omega_{i j}}=-2 \mathrm{u}_{\omega i j} \frac{\partial g^{-1}\left(\eta_{\omega i j}\right)}{\partial \eta_{\omega i j}} \beta_{k} s_{x_{k}}, & &
\end{array}
$$

com $\mathcal{U}_{\omega i j}$ e $\mathcal{E}_{1 \omega i j}$ definidos, respectivamente, em (6.29) e $(6.48), i=1, \ldots, n, j=1, \ldots, t$ e $k$ representa a $k$-ésima coluna de $\mathbf{X}$ com perturbação, $k=2, \ldots, p$.

\section{B.2.1.2 Matriz $\mathrm{Q}$ totalmente igual à matriz $\mathrm{X}$}

BETA 
De (6.49), as derivadas de $\boldsymbol{\Lambda}_{\omega i}, \boldsymbol{\Omega}_{\omega i}$ e $\mathbf{b}_{\omega i}$ todas com relação à $\boldsymbol{\omega}_{i}^{\top}$ são dadas por

$$
\begin{aligned}
& \frac{\partial \boldsymbol{\Lambda}_{\omega i}}{\partial \boldsymbol{\omega}_{i}^{\top}}=\left(\begin{array}{c}
\frac{\partial\left[\mathbf{G}_{\omega i} \boldsymbol{\Phi}_{\omega i} \mathbf{A}_{\omega i}\right]}{\partial \boldsymbol{\omega}_{i}^{\top}} \\
\frac{\partial\left[\mathbf{F}_{\omega i} \mathbf{C}_{\omega i}\right]}{\partial \boldsymbol{\omega}_{i}^{\top}}
\end{array}\right)=\left(\begin{array}{c}
\mathbf{G}_{\omega i} \boldsymbol{\Phi}_{\omega i} \frac{\partial \mathbf{A}_{\omega i}}{\partial \boldsymbol{\omega}_{i}^{\top}}+\mathbf{G}_{\omega i} \frac{\partial \mathbf{\Phi}_{\omega i}}{\partial \boldsymbol{\omega}_{i}^{\top}} \mathbf{A}_{\omega i}+\frac{\partial \mathbf{G}_{\omega i}}{\partial \boldsymbol{\omega}_{i}^{\top}} \boldsymbol{\Phi}_{\omega i} \mathbf{A}_{\omega i} \\
\mathbf{F}_{\omega i} \frac{\partial \mathbf{C}_{\omega i}}{\partial \boldsymbol{\omega}_{i}^{\top}}+\frac{\partial \mathbf{F}_{\omega i}}{\partial \boldsymbol{\omega}_{i}^{\top}} \mathbf{C}_{\omega i}
\end{array}\right), \\
& \frac{\partial \boldsymbol{\Omega}_{\omega i}}{\partial \boldsymbol{\omega}_{i}^{\top}}=\frac{\partial\left[\mathbf{A}_{\omega i}^{1 / 2} \mathbf{R}(\boldsymbol{\alpha}) \mathbf{A}_{\omega i}^{1 / 2}\right]}{\partial \boldsymbol{\omega}_{i}^{\top}}=\mathbf{A}_{\omega i}^{1 / 2} \mathbf{R}(\boldsymbol{\alpha}) \frac{\partial \mathbf{A}_{\omega i}^{1 / 2}}{\partial \boldsymbol{\omega}_{i}^{\top}}+\frac{\partial \mathbf{A}_{\omega i}^{1 / 2}}{\partial \boldsymbol{\omega}_{i}^{\top}} \mathbf{R}(\boldsymbol{\alpha}) \mathbf{A}_{\omega i}^{1 / 2}, \quad \mathrm{e} \\
& \frac{\partial \mathbf{b}_{\omega i}}{\partial \boldsymbol{\omega}_{i}^{\top}}=\frac{\partial\left[\mathbf{y}_{i}^{*}-\boldsymbol{\mu}_{\omega i}^{*}\right]}{\partial \boldsymbol{\omega}_{i}^{\top}}=-\frac{\partial \boldsymbol{\mu}_{\omega i}^{*}}{\partial \boldsymbol{\omega}_{i}^{\top}},
\end{aligned}
$$

sendo as expressões das derivadas de $\mathbf{A}_{\omega i}, \mathbf{G}_{\omega i}$ e $\boldsymbol{\mu}_{\omega i}^{*}$ com relação à $\boldsymbol{\omega}_{i}^{\top}$ dadas na Seção B.1.2 do Apêndice B, $\partial \boldsymbol{\Phi}_{\omega i} / \partial \boldsymbol{\omega}_{i}^{\top}=\operatorname{diag}\left(\partial \phi_{\omega i 1} / \partial \omega_{i 1}, \ldots, \partial \phi_{\omega i t} / \partial \omega_{i t}\right)$ e $\partial \mathbf{C}_{\omega i} / \partial \boldsymbol{\omega}_{i}^{\top}=$ $\operatorname{diag}\left(\partial \mathrm{c}_{\omega i 1} / \partial \omega_{i 1}, \ldots, \partial \mathrm{c}_{\omega i t} / \partial \omega_{i t}\right)$, em que

$$
\begin{aligned}
& \frac{\partial \mathrm{a}_{\omega i j}}{\partial \omega_{i j}}=\mathcal{E}_{1 \omega i j} \frac{\partial g^{-1}\left(\eta_{\omega i j}\right)}{\partial \eta_{\omega i j}} \beta_{k} s_{x_{k}}+\mathcal{E}_{2 \omega i j} \frac{\partial f^{-1}\left(\delta_{\omega i j}\right)}{\partial \delta_{\omega i j}} \gamma_{k} s_{x_{k}} \\
& \frac{\partial}{\partial \omega_{i j}}\left[\frac{\partial g^{-1}\left(\eta_{\omega i j}\right)}{\partial \eta_{\omega i j}}\right]=\frac{\partial^{2} g^{-1}\left(\eta_{\omega i j}\right)}{\partial \eta_{\omega i j}^{2}} \beta_{k} s_{x_{k}}, \quad \frac{\partial}{\partial \omega_{i j}}\left[\frac{\partial f^{-1}\left(\delta_{\omega i j}\right)}{\partial \delta_{\omega i j}}\right]=\frac{\partial^{2} f^{-1}\left(\delta_{\omega i j}\right)}{\partial \delta_{\omega i j}^{2}} \gamma_{k} s_{x_{k}}, \\
& \frac{\partial \mathbf{a}_{\omega i j}^{1 / 2}}{\partial \omega_{i j}}=\frac{1}{2} \mathrm{a}_{\omega i j}^{-1 / 2} \frac{\partial \mathbf{a}_{\omega i j}}{\partial \omega_{i j}}, \quad \frac{\partial \phi_{\omega i j}}{\partial \omega_{i j}}=\frac{\partial f^{-1}\left(\delta_{\omega i j}\right)}{\partial \delta_{\omega i j}} \gamma_{k} s_{x_{k}}, \\
& \frac{\partial \mathrm{c}_{\omega i j}}{\partial \omega_{i j}}=\left[\mathrm{a}_{\omega i j}+\mu_{\omega i j} \mathcal{E}_{1 \omega i j}\right] \frac{\partial g^{-1}\left(\eta_{\omega i j}\right)}{\partial \eta_{\omega i j}} \beta_{k} s_{x_{k}}-\left(1-\mu_{\omega i j}\right) \mathcal{E}_{2 \omega i j} \frac{\partial f^{-1}\left(\delta_{\omega i j}\right)}{\partial \delta_{\omega i j}} \gamma_{k} s_{x_{k}}, \\
& \frac{\partial \mu_{\omega i j}^{*}}{\partial \omega_{i j}}=\mathrm{a}_{\omega i j} \phi_{\omega i j} \frac{\partial g^{-1}\left(\eta_{\omega i j}\right)}{\partial \eta_{\omega i j}} \beta_{k} s_{x_{k}}+\mathcal{E}_{3 \omega i j} \frac{\partial f^{-1}\left(\delta_{\omega i j}\right)}{\partial \delta_{\omega i j}} \gamma_{k} s_{x_{k}},
\end{aligned}
$$

$\operatorname{com} \mathcal{E}_{1 \omega i j}, \mathcal{E}_{2 \omega i j}$ e $\mathcal{E}_{3 \omega i j}$ definidos, respectivamente, em (6.50), (6.51) e (6.52), $i=1, \ldots, n$, $j=1, \ldots, t$ e $k$ representa a $k$-ésima coluna de $\mathbf{X}$ com perturbação, $k=2, \ldots, p$.

\section{SIMPLEX}

De (6.53), as derivadas de $\boldsymbol{\Lambda}_{\omega i}, \boldsymbol{\Omega}_{\omega i}$ e $\mathbf{b}_{\omega i}$ todas com relação à $\boldsymbol{\omega}_{i}^{\top}$ são dadas por

$$
\begin{aligned}
\frac{\partial \boldsymbol{\Lambda}_{\omega i}}{\partial \boldsymbol{\omega}_{i}^{\top}} & =\left(\begin{array}{cc}
\mathbf{G}_{\omega i} \boldsymbol{\Phi}_{\omega i} \frac{\partial \mathbf{A}_{\omega i}}{\partial \boldsymbol{\omega}_{i}^{\top}}+\mathbf{G}_{\omega i} \frac{\partial \mathbf{\Phi}_{\omega i}}{\partial \boldsymbol{\omega}_{i}^{\top}} \mathbf{A}_{\omega i}+\frac{\partial \mathbf{G}_{\omega i}}{\partial \boldsymbol{\omega}_{i}^{\top}} \boldsymbol{\Phi}_{\omega i} \mathbf{A}_{\omega i} & \mathbf{0} \\
\mathbf{0} & \frac{\partial \mathbf{F}_{\omega i}}{\partial \boldsymbol{\omega}_{i}^{\top}}
\end{array}\right), \\
\frac{\partial \boldsymbol{\Omega}_{\omega i}}{\partial \boldsymbol{\omega}_{i}^{\top}} & =\left(\begin{array}{cc}
\mathbf{A}_{\omega i}^{1 / 2} \mathbf{R}(\boldsymbol{\alpha}) \frac{\partial \mathbf{A}_{\omega i}^{1 / 2}}{\partial \boldsymbol{\omega}_{i}^{\top}}+\frac{\partial \mathbf{A}_{\omega i}^{1 / 2}}{\partial \boldsymbol{\omega}_{i}^{\top}} \mathbf{R}(\boldsymbol{\alpha}) \mathbf{A}_{\omega i}^{1 / 2} & \mathbf{0} \\
\mathbf{0} & \frac{\partial \mathbf{D}_{\omega i}}{\partial \boldsymbol{\omega}_{i}^{\top}}
\end{array}\right),
\end{aligned}
$$

$$
\frac{\partial \mathbf{b}_{\omega i}}{\partial \boldsymbol{\omega}_{i}^{\top}}=\left(\begin{array}{c}
\frac{\partial \mathbf{u}_{\omega i}}{\partial \boldsymbol{\omega}_{i}^{\top}} \\
\frac{\partial \mathbf{b}_{2 \omega i}}{\partial \boldsymbol{\omega}_{i}^{\top}}
\end{array}\right),
$$


sendo as expressões das derivadas de $\mathbf{A}_{\omega i}, \mathbf{G}_{\omega i}$ e $\mathbf{u}_{\omega i}$ com relação à $\boldsymbol{\omega}_{i}^{\top}$ dadas na Seção B.1.2 do Apêndice B, $\partial \boldsymbol{\Phi}_{\omega i} / \partial \boldsymbol{\omega}_{i}^{\top}=\operatorname{diag}\left(\partial \phi_{\omega i 1} / \partial \omega_{i 1}, \ldots, \partial \phi_{\omega i t} / \partial \omega_{i t}\right), \partial \mathbf{D}_{\omega i} / \partial \boldsymbol{\omega}_{i}^{\top}=$ $\operatorname{diag}\left(4 \phi_{\omega i 1}^{-1}\left[\partial f^{-1}\left(\delta_{\omega i 1}\right) / \partial \delta_{\omega i 1}\right] \quad \gamma_{k} s_{x_{k}}, \ldots, 4 \phi_{\omega i t}^{-1}\left[\partial f^{-1}\left(\delta_{\omega i t}\right) / \partial \delta_{\omega i t}\right] \gamma_{k} s_{x_{k}}\right) \quad$ e $\quad \partial \mathbf{b}_{2 \omega i} / \partial \boldsymbol{\omega}_{i}^{\top}=$ $\operatorname{diag}\left(\partial \mathrm{b}_{2 \omega i 1} / \partial \omega_{i 1}, \ldots, \partial \mathrm{b}_{2 \omega i t} / \partial \omega_{i t}\right)$, em que

$$
\begin{aligned}
& \frac{\partial \mathrm{a}_{\omega i j}}{\partial \omega_{i j}}=\mathcal{E}_{1 \omega i j} \frac{\partial g^{-1}\left(\eta_{\omega i j}\right)}{\partial \eta_{\omega i j}} \beta_{k} s_{x_{k}}-\phi_{\omega i j}^{2} \mathcal{E}_{2 \omega i j} \frac{\partial f^{-1}\left(\delta_{\omega i j}\right)}{\partial \delta_{\omega i j}} \gamma_{k} s_{x_{k}}, \\
& \frac{\partial}{\partial \omega_{i j}}\left[\frac{\partial g^{-1}\left(\eta_{\omega i j}\right)}{\partial \eta_{\omega i j}}\right]=\frac{\partial^{2} g^{-1}\left(\eta_{\omega i j}\right)}{\partial \eta_{\omega i j}^{2}} \beta_{k} s_{x_{k}}, \quad \frac{\partial}{\partial \omega_{i j}}\left[\frac{\partial f^{-1}\left(\delta_{\omega i j}\right)}{\partial \delta_{\omega i j}}\right]=\frac{\partial^{2} f^{-1}\left(\delta_{\omega i j}\right)}{\partial \delta_{\omega i j}^{2}} \gamma_{k} s_{x_{k}}, \\
& \frac{\partial \mathbf{a}_{\omega i j}^{1 / 2}}{\partial \omega_{i j}}=\frac{1}{2} \mathbf{a}_{\omega i j}^{-1 / 2} \frac{\partial \mathbf{a}_{\omega i j}}{\partial \omega_{i j}} \\
& \frac{\partial \phi_{\omega i j}}{\partial \omega_{i j}}=\frac{\partial f^{-1}\left(\delta_{\omega i j}\right)}{\partial \delta_{\omega i j}} \gamma_{k} s_{x_{k}} \\
& \frac{\partial \mathbf{u}_{\omega i j}}{\partial \omega_{i j}}=\mathcal{U}_{\omega i j} \frac{\partial g^{-1}\left(\eta_{\omega i j}\right)}{\partial \eta_{\omega i j}} \beta_{k} s_{x_{k}} \\
& \frac{\partial \mathrm{b}_{2 \omega i j}}{\partial \omega_{i j}}=-\left[2 \mathrm{u}_{\omega i j} \frac{\partial g^{-1}\left(\eta_{\omega i j}\right)}{\partial \eta_{\omega i j}} \beta_{k} s_{x_{k}}+\frac{\partial f^{-1}\left(\delta_{\omega i j}\right)}{\partial \delta_{\omega i j}} \gamma_{k} s_{x_{k}}\right] \text {, }
\end{aligned}
$$

$\operatorname{com} \mathcal{E}_{1 \omega i j}, \mathcal{E}_{2 \omega i j}$ e $\mathcal{U}_{\omega i j}$ definidos, respectivamente, em (6.54), (6.55) e (6.29), $i=1, \ldots, n$, $j=1, \ldots, t$ e $k$ representa a $k$-ésima coluna de $\mathbf{X}$ com perturbação, $k=2, \ldots, p$. 


\section{Apêndice C}

\section{Tabelas}

Neste apêndice, apresentamos tabelas que detalham algumas das funções definidas neste trabalho.

\section{FunÇÕES G E F}

A Tabela C.1 apresenta algumas das possíveis expressões para $\mathbf{G}$ e $\mathbf{F}$ de acordo, respectivamente, com as funções de ligação $g(\cdot)$ e $f(\cdot)$ adotadas nos componentes sistemáticos definidos na Parte I.

\begin{tabular}{c|cc|cccc}
\hline \multirow{2}{*}{$\begin{array}{c}\text { Função de } \\
\text { ligação }\end{array}$} & \multicolumn{2}{|c|}{ Inversa da } & \multicolumn{4}{c}{ Derivadas de $1^{a}$ e $2^{a}$ ordens } \\
função de ligação & $\mathrm{G}=$ & $\mathrm{F}=$ & $\dot{\mathrm{G}}=$ & $\dot{\mathrm{F}}=$ \\
$g(\cdot)$ ou $f(\cdot)$ & $g^{-1}(\eta)$ & $f^{-1}(\delta)$ & $\frac{\partial g^{-1}(\eta)}{\partial \eta}$ & $\frac{\partial f^{-1}(\delta)}{\partial \delta}$ & $\frac{\partial^{2} g^{-1}(\eta)}{\partial \eta^{2}}$ & $\frac{\partial^{2} f^{-1}(\delta)}{\partial \delta^{2}}$ \\
\hline \hline Identidade & $\eta$ & $\delta$ & 1 & 1 & 0 & 0 \\
Log & $e^{\eta}$ & $e^{\delta}$ & $e^{\eta}$ & $e^{\delta}$ & $e^{\eta}$ & $e^{\delta}$ \\
Logito & $\frac{e^{\eta}}{1+e^{\eta}}$ & $\frac{e^{\delta}}{1+e^{\delta}}$ & $\frac{e^{\eta}}{\left(1+e^{\eta}\right)^{2}}$ & $\frac{e^{\delta}}{\left(1+e^{\delta}\right)^{2}}$ & $\frac{e^{\eta}}{\left(1+e^{\eta}\right)^{4}}$ & $\frac{e^{\delta}}{\left(1+e^{\delta}\right)^{4}}$ \\
Recíproca & $\eta^{-1}$ & $\delta^{-1}$ & $-\eta^{-2}$ & $-\delta^{-2}$ & $-2 \eta^{-3}$ & $-2 \delta^{-3}$ \\
- & $\eta^{-2}$ & $\delta^{-2}$ & $-2 \eta^{-3}$ & $-2 \delta^{-3}$ & $-6 \eta^{-4}$ & $-6 \delta^{-4}$ \\
Compl. $\log -\log$ & $1-e^{-e^{\eta}}$ & $1-e^{-e^{\delta}}$ & $e^{\eta-e^{\eta}}$ & $e^{\delta-e^{\delta}}$ & $\left(1-e^{\eta}\right) e^{\eta-e^{\eta}}$ & $\left(1-e^{\delta}\right) e^{\delta-e^{\delta}}$ \\
\hline
\end{tabular}

Tabela C.1: Funções $\mathbf{G}$ e $\mathbf{F}$ de acordo com a função de ligação adotada 


\section{DistRibUiÇÕES MAIS IMPORTANTES PERTENCENTES À FAMÍliA EXPONENCIAL}

Assumindo que a densidade marginal de y pertence à família exponencial com parâmetro de posição $\varphi$ e de precisão $\phi$, cuja densidade (ou função de probabilidade) dada por

$$
f(\mathrm{y} ; \varphi, \phi)=\exp \{\phi[\mathrm{y} \varphi-b(\varphi)]+c(\mathrm{y} ; \phi)\}
$$

sendo $\phi^{-1}>0, b(\cdot)$ e $c(\cdot)$ funções conhecidas. Dessa forma, a esperança e a variância de y são dadas por $\mathrm{E}(\mathrm{y})=\dot{b}(\varphi)=\mu$ e $\operatorname{Var}(\mathrm{y})=\phi^{-1} \ddot{b}(\varphi)=\phi^{-1} v(\mu)$, sendo $\dot{b}(\varphi)$ e $\ddot{b}(\varphi)$, respectivamente, as derivadas de primeira e segunda ordem de $b(\varphi)$ com relação à $\varphi$. A função $v(\mu)$ é denominada função de variância.

As Tabelas C.2 e C.3 apresentam algumas das distribuições mais importantes que assumem a forma da equação (C.1). Para mais detalhes, veja McCullagh e Nelder (1989) e Paula (2004).

\begin{tabular}{l|ccccc}
\hline & Normal & Poisson & Binomial & Gama & Normal Inversa \\
\hline \hline Notação & $\mathrm{N}\left(\mu, \sigma^{2}\right)$ & $P(\mu)$ & $\mathrm{B}(m, p) / m$ & $\mathrm{G}(\mu ; \nu)$ & $\mathrm{NI}\left(\mu, \sigma^{2}\right)$ \\
Suporte de y & $(-\infty ; \infty)$ & $\{0,1, \ldots\}$ & $\{0,1, \ldots, m\} / m$ & $(0 ; \infty)$ & $(0 ; \infty)$ \\
$\phi^{-1}$ & $\sigma^{2}$ & 1 & $1 / m$ & $\nu^{-1}$ & $\sigma^{2}$ \\
$\varphi$ & $\mu$ & $\log (\mu)$ & $\log \left(\frac{\mu}{1-\mu}\right)$ & $\mu^{-1}$ & $\mu^{-2}$ \\
$b(\varphi)$ & $\varphi^{2} / 2$ & $e^{\varphi}$ & $\log \left(1+e^{\varphi}\right)$ & $-\log (-\varphi)$ & $-(-2 \varphi)^{1 / 2}$ \\
$\mu=\dot{b}(\varphi)$ & $\varphi$ & $e^{\varphi}$ & $\frac{e^{\varphi}}{1+e^{\varphi}}$ & $-\varphi^{-1}$ & $(-2 \varphi)^{-1 / 2}$ \\
$v(\mu)$ & 1 & $\mu$ & $\mu(1-\mu)$ & $\mu^{2}$ & $\mu^{3}$ \\
Lig. canônica & identidade & $\log$ & $\operatorname{logito}$ & recíproca & - \\
\hline
\end{tabular}

Tabela C.2: Algumas distribuições pertencentes à família exponencial e respectivos resultados 
FUNÇÕES $c(\mathrm{Y} ; \phi), \dot{c}(\mathrm{Y} ; \phi)$ E $\ddot{c}(\mathrm{Y} ; \phi)$

\begin{tabular}{l|ccc}
\hline & $c(\mathrm{y} ; \phi)$ & $\dot{c}(\mathrm{y} ; \phi)$ & $\ddot{c}(\mathrm{y} ; \phi)$ \\
\hline \hline Normal & $-\frac{1}{2}\left[\phi \mathrm{y}^{2}+\log \left(\frac{2 \pi}{\phi}\right)\right]$ & $-\frac{1}{2}\left[\mathrm{y}^{2}-\phi^{-1}\right]$ & $1 / 2$ \\
Poisson & $-\log (\mathrm{y} !)$ & - & - \\
Binomial & $\log \left(\begin{array}{c}\phi \\
\phi \mathrm{y}\end{array}\right)$ & - & - \\
Gama & $(\phi-1) \log \mathrm{y}+\phi \log \phi-\log \Gamma(\phi)$ & $1+\log (\phi \mathrm{y})-\psi(\phi)$ & $\phi^{2} \psi^{\prime}(\phi)-\phi$ \\
Normal Inversa & $-\frac{1}{2}\left[\phi \mathrm{y}^{-1}+\log \left(\frac{2 \pi \mathrm{y}^{3}}{\phi}\right)\right]$ & $-\frac{1}{2}\left[\mathrm{y}^{-1}-\phi^{-1}\right]$ & $1 / 2$ \\
\hline
\end{tabular}

Tabela C.3: Funções $c(\mathrm{y} ; \phi), \dot{c}(\mathrm{y} ; \phi)$ e $\ddot{c}(\mathrm{y} ; \phi)$ de acordo com a distribuição adotada

\section{Alguns ReSUltados PARA INFLUÊNCIA LOCAL QUANDO ABORDAmos MLGs}

A Tabela C.4 apresenta algumas derivadas utilizadas na construção das medidas de influência local quando consideramos modelos lineares generalizados.

\begin{tabular}{c|ccccc}
\hline Derivadas & Normal & Poisson & Binomial & Gama & Normal Inversa \\
\hline \hline$\left.\frac{\partial v\left(\mu_{\omega}\right)}{\partial \mu_{\omega}}\right|_{\omega_{0}}$ & 0 & 1 & $1-2 \mu$ & $2 \mu$ & $3 \mu^{2}$ \\
$\left.\frac{\partial \dot{c}\left(\mathrm{y}_{\omega} ; \phi\right)}{\partial \omega}\right|_{\omega_{0}}$ & $-\mathrm{y} \sqrt{\operatorname{Var}(\mathrm{y})}$ & - & - & $\frac{\sqrt{\operatorname{Var}(\mathrm{y})}}{\mathrm{y}}$ & $\frac{\sqrt{\operatorname{Var}(\mathrm{y})}}{2 \mathrm{y}^{2}}$ \\
$\left.\frac{\partial b\left(\varphi_{\omega}\right)}{\partial \varphi_{\omega}}\right|_{\omega_{0}}$ & $\varphi$ & - & - & $\frac{1}{\varphi}$ & $-2 \varphi^{-1 / 2}$ \\
$\left.\frac{\partial \varphi_{\omega}}{\partial \mu_{\omega}}\right|_{\omega_{0}}$ & 1 & - & - & $\mu^{-2}$ & $\mu^{-3}$ \\
$\left.\frac{\partial \mathrm{E}\left(\ddot{c}\left(\mathrm{y}_{\omega} ; \phi\right)\right)}{\partial \phi_{\omega}^{-1}}\right|_{\omega_{0}}$ & 0 & - & - & $-\phi^{2}\left(2 \phi \psi^{\prime}(\phi)+\right.$ & 0 \\
\hline
\end{tabular}

Tabela C.4: Alguns resultados para influência local quando abordamos MLGs 


\section{Referências Bibliográficas}

Akaike, H. (1973). Information theory and an extension of the maximum likelihood principle, B. n. petrov. and f. csaki (eds), In Proceedings of the Second International on Information Theory, Budapest: Akademiai Kiado.

Andreoni, S. (1989). Modelos de efeitos aleatórios para análise de dados longitudinais não balanceados em relação ao tempo, Dissertação de mestrado, IME-USP, São Paulo. $142 \mathrm{p}$.

Artes, R. (1997). Extensões da teoria das equações de estimação generalizadas a dados circulares e modelos de dispersão, Tese de doutorado, IME-USP, São Paulo. 130p.

Artes, R. e Botter, D. A. (2005). Funções de Estimação em Modelos de Regressão, $9^{a}$ Escola de Modelos de Regressão - ABE, São Pedro - SP.

Artes, R. e J $\phi$ rgensen, B. (2000). Longitudinal data estimating equations for dispersion models, Scandinavian Journal of Statistics 27: 321-334.

Atkinson, A. C. (1985). Plots, Transformations and Regressions, Oxford Statistical Science Series, Oxford.

Barndorff-Nielsen, O. E. e Jorgensen, B. (1991). Some parametric models on the simplex, Journal of Multivariate Analysis 39: 106-116.

Bates, D. M. e Watts, D. G. (1980). Relative curvature measures of non-linearity, $J$. $R$. Statist. Soc. B 42: 1-25.

Botter, D., Sandoval, M., Iwamoto, E. e Bonassi, F. (2006). Desenvolvimento motor dos recém-nascidos pré-termo e a termo até aquisição da marcha segundo aberta infant motor scale: um estudo de coorte, Relatório técnico, São Paulo: IME - USP, RAE-CEA-06P20.

Cadigan, N. G. (1995). Local influence in structural equation models, Structural Equation Modeling 2 (1): 13-30. 
Cadigan, N. G. e Farrell, P. J. (2002). Generalized local influence with applications to fish stock cohort analysis, Appl. Statist. 51 (4): 469-483.

Chandrasekar, B. e Kale, B. K. (1984). Unbiased statistical estimation functions in presence of nuisance parameter, Journal of Statistical Planning and Inference 9: 45-54.

Cook, R. D. (1977). Detection of influential observations in linear regressions, Technometrics 19: 15-18.

Cook, R. D. (1986). Assessment of local influence, J. R. Statist. Soc. 48 (2): 133-169.

Crowder, M. (1987). On linear and quadratic estimating function, Biometrika 74: 591597.

Espinheira, P. L., Ferrari, S. L. P. e Cribari-Neto, F. (2008). On beta regression residuals, Journal of Applied Statistics 35: 407-419.

Ferrari, S. L. P. e Cribari-Neto, F. (2004). Beta regression for modeling rates and proportions, Journal of Applied Statistics 31 (7): 799-815.

Godambe, V. P. (1960). An optimum property of regular maximum likelihood estimation, Annals of Mathematical Statistics 31: 1208-1211.

Graybill, F. A. (1976). Theory and Application of the Linear Model, Wadsworth Publishing Company, California.

Harville, D. A. (1997). Matrix Algebra From a Statistician's Perspective, Springer, New York.

Joe, H. (1997). Multivariate Models and Dependence Concepts, Chapamn \& Hall, London.

Johnston, G. (1996). Repeated measures analysis with discrete data using the sas system, SAS Institute Inc.

URL: http://academic.son.wisc.edu/rdsu/pdf/gee.pdf

J $\phi$ rgensen, B. (1997). The Theory of Dispersion Models, Chapman \& Hall, London.

J $\phi$ rgensen, B. e Labouriau, R. S. (1994). Exponential families and theoretical inference, Lecture notes, University of British Columbia.

Kullback, S. e Leibler, R. A. (1951). On information and sufficiency, Annals of Mathematical Statistics 22: 79-86.

Laird, N. M. e Ware, J. H. (1982). Random-effects models for longitudinal data, Biometrics 38: 963-974. 
Liang, K.-Y. e Zeger, S. L. (1986). Longitudinal analysis using generalized linear models, Biometrika 73: 13-22.

McCullagh, P. e Nelder, J. A. (1989). Generalized Linear Models, 2nd edn, Chapman and Hall, London.

Meyers, S. M., Ambler, J. S., Tan, M., Werner, J. C. e Huang, S. S. (1992). Variation of perfluorpropane disappearance after vitrectomy, Retina 12: 359-363.

Nelder, J. A. e Wedderburn, R. W. M. (1972). Generalized linear models, Journal of the Royal Statistical Society A 135: 370-384.

Neter, J., Kutner, M. H., Naschstheim, C. J. e Wasserman, W. (1996). Applied Linear Statistical Models, IE McGraw Hill, Chicago.

Oliveira, M. S. (2004). Um modelo de regressão beta: teoria e aplicações, Dissertação de mestrado, IME-USP, São Paulo. 99p.

Ospina, P. L. E. (2007). Regressão beta, Tese de doutorado, IME-USP, São Paulo. 158p.

Ospina, R. e Ferrari, S. L. P. (2008). Inflated beta distributions, Statistical Papers 49: 117.

Pan, W. (2001). Akaike's information criterion in generalized estimating equations, Biometrics 57: 120-125.

Park, C. G. e Shin, D. W. (1998). An algorithm for generating correlated random variables in a class of infinitely divisible distributions, J. Statist. Comput. Simul. 61: 127-139.

Paula, G. A. (2004). Modelos de regressão com apoio computacional, Notas de aulas, Departamento de Estatística, Universidade de São Paulo.

\section{URL: http://www.ime.usp.br/ giapaula/mlgs.html}

Pregibon, D. (1981). Logistic regression diagnostics, Annals of Statistics 9: 705-724.

Preisser, J. S. e Perin, J. (2007). Deletion diagnostics for marginal mean and correlation model parameters in estimating equations, Stat Comput 17: 381-393.

Prentice, R. L. (1988). Correlated binary regression with covariates specific to each binary observation, Biometrics 44: 1033-1048.

Prentice, R. L. e Zhao, L. P. (1991). Estimating equations for parameters in means and covariances of multivariate discrete and continuous responses, Biometrics 47: 825839. 
Sen, P. K. e Singer, J. M. (1993). Large Sample Methods in Statistics: an introduction with applications, Chapman \& Hall, New York.

Singer, J. M. e Andrade, D. F. (1986). Análise de Dados Longitudinais, VII Simpósio Nacional de Probabilidade e Estatística, São Paulo: Campinas. 106p.

Song, P. X.-K., Qiu, Z. e Tan, M. (2004). Modelling heterogeneous dispersion in marginal models for longitudinal proportional data, Biometrical Journal 46: 5, 540-553.

Song, P. X.-K. e Tan, M. (2000). Marginal models for longitudinal continuous proportional data, Biometrics 56: 496-502.

Thomas, W. e Cook, R. D. (1989). Assessing influence on regression coefficients in generalized linear models, Biometrika 76: 741-749.

Thomas, W. e Cook, R. D. (1990). Assessing influence on predictions from generalized linear models, Technometrics 32: 59-65.

Venezuela, M. K. (2003). Modelos lineares generalizados para análise de dados com medidas repetidas, Dissertação de mestrado, IME-USP, São Paulo. 95p.

Venezuela, M. K., Botter, D. A. e Sandoval, M. C. (2007). Diagnostic techniques in generalized estimating equations, Journal of Statistical Computation and Simulation 77: 879-888.

Wang, Y.-G. e Carey, V. (2003). Working correlation structure misspecification, estimation and covariate design: Implications for generalised estimating equations performance, Biometrika 90: 29-41.

Wedderburn, R. W. M. (1974). Quasi-likelihood functions, generalized linear models, and the gauss-newton method, Biometrika 61: 31-38.

Wei, B.-C., Hu, Y.-Q. e Fung, W.-K. (1998). Generalized leverage and its applications, Scandinavian Journal of Statistics 25: 25-37.

Zeger, S. L. e Liang, K.-Y. (1986). Longitudinal data analysis for discrete and continuous outcomes, Biometrics 42: 121-130. 